Solução numérica de escoamentos de cristais

líquidos nemáticos

\author{
Pedro Alexandre da Cruz
}



Data de Depósito:

Assinatura:

\title{
Solução numérica de escoamentos de cristais líquidos nemáticos
}

\author{
Pedro Alexandre da Cruz
}

Orientador: Prof. Dr. Murilo Francisco Tomé

Tese apresentada ao Instituto de Ciências Matemáticas e de Computação - ICMC-USP, como parte dos requisitos para obtenção do título de Doutor em Ciências - Ciências de Computação e Matemática Computacional. VERSÃO REVISADA

USP - São Carlos

Agosto de 2011 
Ficha catalográfica elaborada pela Biblioteca Prof. Achille Bassi e Seção Técnica de Informática, ICMC/USP, com os dados fornecidos pelo(a) autor(a)

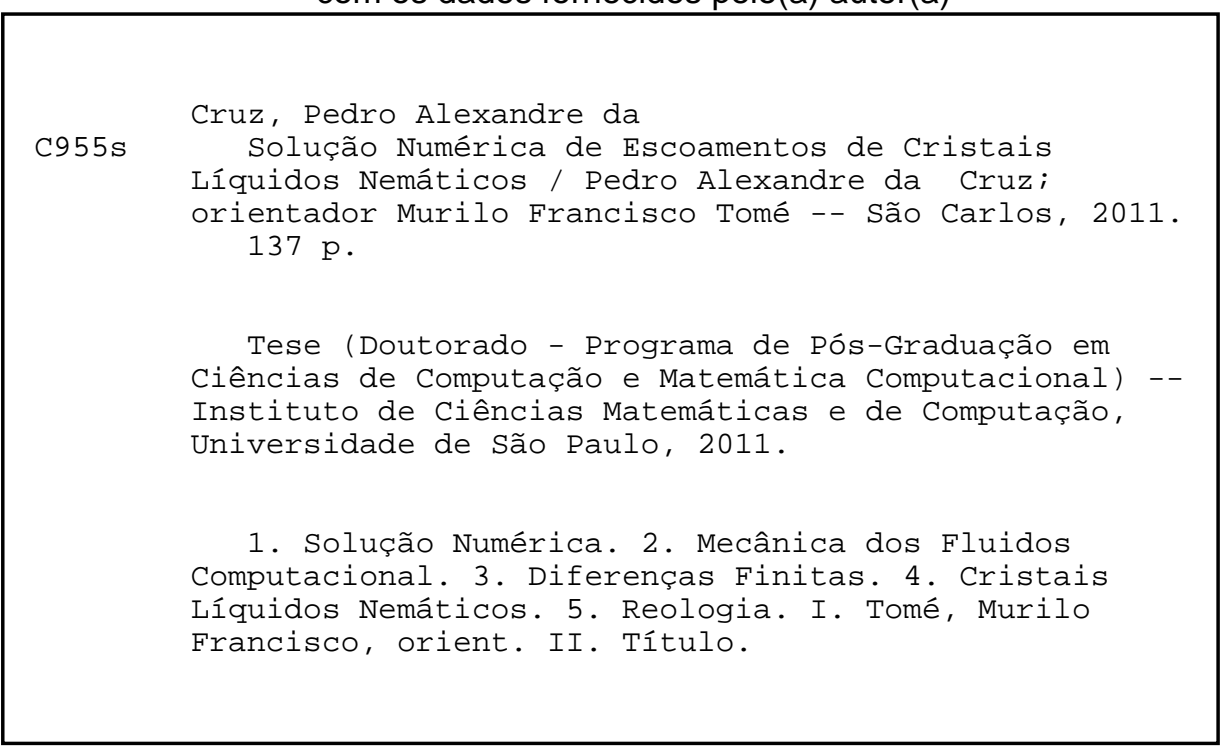


"Que os nossos esforços desafiem as impossibilidades. Lembrai-vos de que as grandes proezas da história foram conquistas do que parecia impossível". Charles Chaplin 

Dedico às pessoas que mais amo na vida: minha família. 



\section{Agradecimentos}

Deixo aqui expresso os meus mais sinceros agradecimentos:

- Ao Prof. Murilo Francisco Tomé pela orientação, apoio, disponibilidade, compreensão e amizade ao longo destes anos.

- A todos os professores do grupo de pesquisa do LCAD que sempre presentes estiveram auxiliando-me com sugestões para o desenvolvimento do projeto.

- Aos professores João Miguel Nóbrega, Olga Carneiro e Vera Machado Nóbrega do Departamento de Engenharia de Polímeros da Universidade do Minho (UMinho), Guimarães, Portugal, pelo suporte e acolhimento durante meu estágio de doutorado e pela oportunidade que deram-me de conhecer vossos ambientes de pesquisa.

- Aos professores Sean McKee e Iain W. Stewart pelas sugestões para o desenvolvimento do projeto.

- Aos amigos e amigas do LCAD, amigos e amigas da Universidade do Minho e colegas que acompanharam os momentos alegres e as dificuldades neste período, sempre me apoiando e incentivando.

- À minha família que sempre esta presente em minha vida e, em especial à minha mãe e meu pai.

- À minha namorada e sua família pela compreensão e apoio durante todo este trabalho.

- À Fapesp pelo financiamento durante todo o desenvolvimento dessa pesquisa e ao programa de cooperação entre o Brasil e Portugal - CAPES-FCT, pelo suporte financeiro durante meu estágio de doutorado no exterior.

- Finalmente, agradeço a Deus por essa oportunidade em minha vida, e acima de tudo, agradeço pela minha vida. 


\section{Resumo}

O objetivo desse trabalho é desenvolver métodos numéricos para simular escoamentos de cristais líquidos nemáticos governados pelas equações dinâmicas de Ericksen-Leslie. São apresentados dois métodos numéricos para a simulação de escoamentos de cristais líquidos nemáticos. O primeiro método foi desenvolvido para simular escoamentos tridimensionais de cristais líquidos nemáticos sob efeito de forte campo magnético enquanto que o segundo método foi desenvolvido para a simulação de escoamentos bidimensionais. Utilizando a notação de Einstein, as equações dinâmicas de Ericksen-Leslie são apresentadas. Empregando variáveis primitivas e coordenadas cartesianas, as equações governantes para escoamentos de cristais líquidos nemáticos são derivadas e as formulações matemáticas para a obtenção dos métodos numéricos são apresentadas. As equações descrevendo os métodos numéricos são resolvidas por um método numérico baseado na metodologia GENSMAC3D para o caso tridimensional enquanto que o método bidimensional é baseado na metodologia GENSMAC (GENeralized-Simplified-Marker-And-Cell). Em ambos os métodos, a técnica de diferenças finitas em uma malha deslocada é utilizada. As equações que descrevem as técnicas numéricas desenvolvidas foram incorporadas aos ambientes de simulação Freeflow2D e Freeflow3D. As condições de contorno para cada tipo de contorno são descritas em detalhes. A solução analítica apresentada por Stewart para o escoamento entre duas placas paralelas é utilizada para a validação do método numérico tridimensional. Empregando as hipóteses de escoamento desenvolvido e que o ângulo de orientação do diretor é pequeno, uma solução analítica para o escoamento em um canal bidimensional é encontrada. O método numérico bidimensional é então validado utilizando a solução analítica obtida. Utilizando refinamento de malha, resultados de convergência dos métodos numéricos são apresentados. Os métodos numéricos desenvolvidos nesse trabalho são aplicados para a simulação dos seguintes problemas: escoamento de um cristal líquido nemático em um canal tridimensional; investigação numérica do escoamento em L-canais e escoamento através de uma contração 4:1 e de uma expansão planar 4:1.

Palavras-chave: Equações dinâmicas de Ericksen-Leslie, Cristais líquidos nemáticos, Diferenças finitas, Solução analítica. 


\section{Abstract}

The aim of this work is to develop numerical methods capable of simulating nematic liquid crystal flows described by the dynamic Ericksen-Leslie equations. Two numerical techniques have been proposed: a numerical method for solving three-dimensional flows of nematic liquid crystals under strong magnetic fields and a method for simulating flows of nematic liquid crystals in two-dimensional complex geometries. Both methods employ Cartesian coordinates using primitive variables of pressure and velocity. These techniques are based on the GENSMAC (GENeralized-Simplified-Marker-And-Cell) methodology and a detailed description of the equations involved is presented. The resulting governing equations are solved by the finite difference method on a staggered grid. The three-dimensional technique was applied to solve fully developed flow between two paralel plates for which an analytic solution exists. By using this analytic solution, validation and convergence results of the developed numerical technique were obtained. To validate the two-dimensional method developed herein, an analytic solution for steady state flow in a 2D-channel was found which was used to obtain validation and convergence results. The method was then applied to simulate the flow in L-shaped channels, flow through a 4:1 contraction and flow in a 4:1 expansion.

Keywords: Ericksen-Leslie dynamic equations, Nematic liquid crystals, Analytic solution, Finite difference 


\section{Sumário}

1 Introdução $\quad 1$

1.1 O que é um Cristal Líquido? . . . . . . . . . . . . . . . . . . . . 1

1.2 A origem dos cristais líquidos . . . . . . . . . . . . . . 3

1.3 Aplicações dos Cristais Líquidos . . . . . . . . . . . . . . . . . . . . . . 4

1.4 Conceitos Introdutórios da Teoria dos Cristais Líquidos . . . . . . . . . . . . 6

1.4.1 Elasticidade . . . . . . . . . . . . . . . . . 8

1.4 .2 Viscosidades de Miesowicz . . . . . . . . . . . . . . . . . . 9

1.4.3 Ancoramento . . . . . . . . . . . . . . . . . . . 10

1.5 Teoria Dinâmica dos Cristais Líquidos Nemáticos . . . . . . . . . . . . . . 11

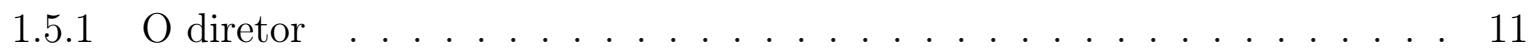

1.5.2 Equações Dinâmicas de Ericksen-Leslie . . . . . . . . . . . . . . . . . 12

1.5.3 As viscosidades de Leslie . . . . . . . . . . . . . . . . . . . . . . 14

1.6 Uma Revisão sobre Escoamentos de Cristais Líquidos . . . . . . . . . . . . . . 15

1.7 Uma Revisão sobre o Método GENSMAC . . . . . . . . . . . . . . . . . . . . . 17

1.8 Linhas Gerais da Tese . . . . . . . . . . . . . . . . . . . . . . . . 18

2 Escoamentos de Cristais Líquidos Nemáticos sob Forte Campo Magnético 21

2.1 Escoamento entre Duas Placas Paralelas . . . . . . . . . . . . . . . . . . . . . 22

2.2 Equações Dinâmicas para um Forte Campo Magnético . . . . . . . . . . . . . . 23

2.2.1 Equações de Conservação de Massa e de Quantidade de Movimento . . . 23

2.2.2 Caso especial para $\phi_{0} \equiv 0 \ldots \ldots \ldots \ldots \ldots$

2.3 Forma Adimensional das Equações Dinâmicas . . . . . . . . . . . . . . . . 26

2.4 Método Numérico . . . . . . . . . . . . . . . . . . . . . . . . . . 27

2.5 Condições de Contorno . . . . . . . . . . . . . . . . . . . . . . . . . . . . . 29

2.6 Método de Solução . . . . . . . . . . . . . . . . . . . . . . . . . . . 29

2.7 Aproximação das Equações por Diferenças Finitas . . . . . . . . . . . . . . . . 31

2.7.1 Cálculo das Componentes do Tensor das Tensões não-newtoniano . . . . 33 
2.7.2 Cálculo do Passo Temporal . . . . . . . . . . . . . . . . . 36

2.8 Verificação do Método Numérico . . . . . . . . . . . . . . . . . . . . . . . . . . 37

2.8.1 Solução Analítica do Escoamento entre Duas Placas . . . . . . . . . . . . 37

2.8.2 Comparação entre as Soluções Numéricas e Analítica . . . . . . . . . . . 39

2.8.3 Avaliação da Solução Analítica . . . . . . . . . . . . . . . . . . 45

2.8.4 Simulação Numérica do Escoamento em um Canal Tridimensional . . . . 51

3 Método Numérico para Escoamentos de Cristais Líquidos Nemáticos em Duas Dimensões $\quad 55$

3.1 Equações Dinâmicas de Ericksen-Leslie em Duas Dimensões . . . . . . . . . . . 55

3.1.1 Equações de Conservação de Massa e de Quantidade de Movimento . . . 56

3.1.2 Equações de Conservação de Momento Angular . . . . . . . . . . . . . . 58

3.1.3 Equações Dinâmicas Adimensionais . . . . . . . . . . . . . . . . . 59

3.2 Método Numérico 2D . . . . . . . . . . . . . . . . . . . . . . . . . . 61

3.2 .1 Condições de Contorno . . . . . . . . . . . . . . . . . 63

3.2 .2 Procedimento Numérico 2D . . . . . . . . . . . . . . . . . . . 63

3.3 Aproximação das Equações por Diferenças Finitas . . . . . . . . . . . . . . . . . 64

3.3.1 Aproximação do Tensor das Tensões não-newtoniano . . . . . . . . . . . 67

3.4 Cálculo do Passo Temporal . . . . . . . . . . . . . . . . . . . . . . . . . . 69

3.5 Validação do Método Numérico Bidimensional . . . . . . . . . . . . . . . 70

3.5.1 Solução Analítica para Escoamentos Desenvolvidos 2D . . . . . . . . . . 70

3.5.2 Comparação entre as Soluções Numéricas e Analíticas . . . . . . . . . . . 74

4 Simulação Numérica de Escoamentos Bidimensionais de Cristais Líquidos $\begin{array}{ll}\text { Nemáticos } & 83\end{array}$

4.1 Investigação Numérica do Escoamento em L-canais . . . . . . . . . . . . . . . 83

4.1.1 Efeito das condições de contorno para o ângulo $\phi$. . . . . . . . . . . 84

4.1.2 Estudo do número de Ericksen e mudança de geometria do canal . . . . . 90

4.2 Simulação Numérica do Escoamento através de uma Contração Planar 4:1 . . 94

4.3 Simulação Numérica do Escoamento de um Cristal Líquido Nemático em uma Expansão . . . . . . . . . . . . . . . . . . . . . . . . . 98

5 Considerações Finais $\quad 119$

$\begin{array}{ll}\text { Referências Bibliográficas } & 123\end{array}$

A Tabelas Importantes 135 


\section{Lista de Figuras}

1.1 Transição de fase devido ao aumento de temperatura dos estados da matéria: sólido cristalino, cristal líquido, líquido isotrópico e gás. . . . . . . . . . . . . . 3

1.2 Mostrador de cristal liquido torcido. Essa figura foi extraída do trabalho de Mottram e Hogan [96]. . . . . . . . . . . . . . . . . . . . . . . 5

1.3 A direção média das moléculas é representada pelo vetor unitário conhecido como diretor. . . . . . . . . . . . . . . . . . 7

1.4 Representação das fases esmética A e esmética C . . . . . . . . . . . . . 8

1.5 Tipos de deformações elásticas básicas: (a) afunilamento (splay) $\left(K_{1}\right)$, (b) torção

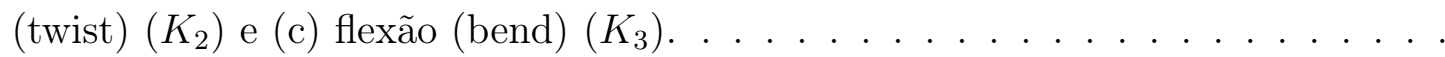

1.6 As viscosidades de Miesowicz: (a) viscosidade cisalhante $\eta_{1}(\mathbf{n} \| \mathbf{u})$, (b) viscosidade cisalhante $\eta_{2}(\mathbf{n} \| \nabla \mathbf{u})$, (c) viscosidade cisalhante $\eta_{3}(\mathbf{n} \perp \mathbf{u}$ e $\mathbf{n} \perp \nabla \mathbf{u})$ e (d) viscosidade rotacional $\gamma_{1}$. A velocidade $\mathbf{u}$ é representada pelas setas. O diretor $\mathbf{n}$ é representado pelo bastão laranja.

1.7 Dois possíveis tipos de forte ancoragem molecular: (a) Orientação Planar e (b) Orientação Homeotrópica. . . . . . . . . . . . . . . . . . . . . . . . . . . . 10

1.8 Representação espacial do diretor $\mathbf{n}$ em termos dos ângulos $\theta$ e $\phi$. . . . . . . . . 12

1.9 As fotos mostram um persiana de LCD. No estado "OFF" sem nenhuma tensão aplicada o cristal líquido espalha a luz incidente e a persiana fica opaca, aplicando uma tensão (estado "ON"), as moléculas de cristal líquido se alinham e a persiana torna-se transparente. . . . . . . . . . . . . . . . . . . . . . 17

2.1 Descrição do escoamento 3D entre duas placas paralelas. . . . . . . . . . . . . 22

2.2 Típica célula tridimensional. . . . . . . . . . . . . . . . . . . . . . 31

2.3 Representação do ângulo de deflexão $\varphi$. . . . . . . . . . . . . . . . . . . . . . 38

2.4 Definição do domínio de escoamento para a simulação do escoamento tridimensional entre duas placas paralelas. As paredes cinzas representam as paredes laterais fictícias. 
2.5 Isolinhas da velocidade $v$ em $t=10$ s: curvas de nível obtidas em vários planos ao longo das placas. - $\phi_{0}=30^{\circ}$. Entrada de fluido pela parte inferior. . . . . . .

2.6 Isolinhas da velocidade $v$ em $t=50$ s: curvas de nível obtidas em vários planos ao longo das placas. - $\phi_{0}=30^{\circ}$. Entrada de fluido pela parte inferior. . . . . . .

2.7 Isolinhas da velocidade $v$ em $t=200$ s: curvas de nível obtidas em vários planos ao longo das placas. - $\phi_{0}=30^{\circ}$. Entrada de fluido pela parte inferior. . . . . . . 42

2.8 Isolinhas da velocidade $w$ em $t=10 \mathrm{~s}$ : curvas de nível obtidas em vários planos ao longo das placas. - $\phi_{0}=30^{\circ}$. Entrada de fluido pela parte inferior. . . . . . . 43

2.9 Isolinhas da velocidade $w$ em $t=50 \mathrm{~s}$ : curvas de nível obtidas em vários planos ao longo das placas. - $\phi_{0}=30^{\circ}$. Entrada de fluido pela parte inferior. . . . . . .

2.10 Isolinhas da velocidade $w$ em $t=200 \mathrm{~s}$ : curvas de nível obtidas em vários planos ao longo das placas. $-\phi_{0}=30^{\circ}$. Entrada de fluido pela parte inferior. . . . . . .

2.11 Isolinhas da pressão $p$ em $t=200$ s: curvas de nível obtidas em vários planos ao longo das placas. Entrada de fluido pela parte inferior. . . . . . . . . . . . 44

2.12 Soluções numéricas e analítica das velocidades $v$ e $w$ diversos valores do ângulos $\phi_{0} \ldots \ldots \ldots \ldots \ldots \ldots \ldots \ldots \ldots$

2.13 Variação do ângulo de deflexão $\varphi$ em função do ângulo $\phi$. Comparação entre resultados numéricos e valores analíticos dado pela equação $(2.83)$. . . . . . . .

2.14 Comparação entre os resultados numéricos e analíticos das componentes do tensor das tensões não newtoniano $\Phi^{x y}, \Phi^{y x}, \Phi^{x z}$ e $\Phi^{z x}$ : Ângulo $\phi_{0}=0^{\circ} . \ldots$. . . . 48

2.15 Comparação entre os resultados numéricos e analíticos das componentes do tensor das tensões não newtoniano $\Phi^{x y}, \Phi^{y x}, \Phi^{x z}$ e $\Phi^{z x}$ : Ângulo $\phi_{0}=30^{\circ} \ldots$. . . . .

2.16 Comparação entre os resultados numéricos e analíticos das componentes do tensor das tensões não newtoniano $\Phi^{x y}, \Phi^{y x}, \Phi^{x z}$ e $\Phi^{z x}$ : Ângulo $\phi_{0}=60^{\circ} \ldots$. . . . .

2.17 Isolinhas da velocidade $v$ em $t=10 \mathrm{~s}$ : curvas de nível obtidas em vários planos ao longo do canal tridimensional. - $\phi_{0}=30^{\circ}$. Entrada de fluido pela parte inferior. 51

2.18 Isolinhas da velocidade $v$ em $t=200 \mathrm{~s}$ : curvas de nível obtidas em vários planos ao longo do canal tridimensional. - $\phi_{0}=30^{\circ}$. Entrada de fluido pela parte inferior. 52

2.19 Isolinhas da velocidade $w$ em $t=10 \mathrm{~s}$ : curvas de nível obtidas em vários planos ao longo do canal tridimensional.- $\phi_{0}=30^{\circ}$. Entrada de fluido pela parte inferior. 53

2.20 Isolinhas da velocidade $w$ em $t=200 \mathrm{~s}$ : curvas de nível obtidas em vários planos ao longo do canal tridimensional. - $\phi_{0}=30^{\circ}$. Entrada de fluido pela parte inferior. 53

2.21 Isolinhas da pressão $p$ em $t=200 \mathrm{~s}$ : curvas de nível obtidas em vários planos ao longo do canal tridimensional. - $\phi_{0}=30^{\circ}$. Entrada de fluido pela parte inferior.

3.1 Típica célula deslocada bidimensional.

3.2 Descrição do escoamento em um Canal 2D. As setas vermelhas representam as condições de contorno utilizadas para o cálculo do ângulo $\phi$. . . . . . . . . . . 71

3.3 Isolinhas de: (a) $u$, (b) $v$ e (c) $p$ em $t=80$ s. . . . . . . . . . . . . 76 
3.4 Simulação numérica do escoamento de um cristal líquido nemático em um canal. Variação de $p(x, y c)$ e $\phi\left(x, y_{c}\right), 0 \leq x \leq 20, y_{c}=0.5+0.5 \delta y$, no tempo $t=80 \mathrm{~s}$. Note que $x$ e $y$ foram adimensionalizadas pela largura do canal $W \ldots$. . . . .

3.5 Simulação numérica do escoamento de um cristal líquido nemático em um canal. Variação de $u\left(x_{c}, y\right)$ e $v\left(x_{c}, y\right), 0 \leq y \leq 1, x_{c}=10+0.5 \delta x$, no tempo $t=80 \mathrm{~s}$. Note que $x$ e $y$ foram adimensionalizadas pela largura do canal $W \ldots \ldots$. . . 78

3.6 Decaimento dos erros relativos com o refinamento da malha: (a) componente $u$ da velocidade e ângulo do diretor $\phi$ e (b) componentes do tensor não-newtoniano $\Phi_{x x}, \Phi_{x y}, \Phi_{y x}, \mathrm{e} \Phi_{y y} \ldots \ldots \ldots \ldots \ldots \ldots$

3.7 Solução numérica e analítica da componente $u\left(x_{c}, y\right), x_{c}=10$, da velocidade para $R e=0.0132$ e $E r=10 \ldots \ldots \ldots \ldots \ldots \ldots$

3.8 Comparação entre os resultados numéricos e analítico das componentes do tensor das tensões não-newtoniano $\Phi_{x y}\left(x_{c}, y\right), \Phi_{y x}\left(x_{c}, y\right), \Phi_{x x}\left(x_{c}, y\right)$ e $\Phi_{y y}\left(x_{c}, y\right), x_{c}=10.80$

3.9 Comparação das soluções numérica e analítica do ângulo do diretor $\phi\left(x_{c}, y\right), x_{c}=$ 10 para $R e=0.0132$ e $E r=10$ nas malhas $\mathrm{M}_{0}, \mathrm{M}_{1}, \mathrm{M}_{2}$. . . . . . . . . .

3.10 Comparação das soluções numéricas e analítica para a primeira diferença de tensões $\sigma=u_{, y}\left(\alpha_{1}+\alpha_{5}-\alpha_{6}\right) \operatorname{sen} \phi \cos \phi$ (à esquerda) e comparação dos tensores $\Phi_{x y}$ e $\Phi_{y x}$ (à direita).

3.11 Gráfico da soluções analíticas para a equação (3.78) obtida na Seção 3.5.1.2 para vários números de Ericksen.

3.12 Comparação das soluções numéricas obtidas na saída do canal com a solução analítica dada pela equação (3.78) para: $E r=20$ e $E r=35$. . . . . . . . . .

4.1 Definição do domínio de simulação do escoamento em um L-canal. As setas vermelhas representam as condições de contorno utilizadas para o cálculo do

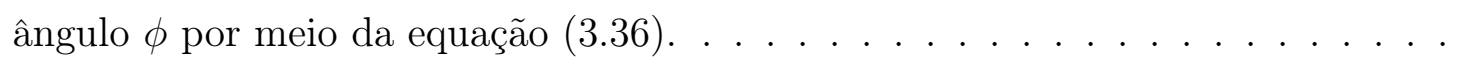

4.2 Simulação Numérica do escoamento através de um L-canal com $R e=0.026$ e $E r=5$. Isolinhas: (a) Pressão, (b) velocidade $u$ e (c) velocidade $v . \quad \ldots . .86$

4.3 Variação da pressão no L-canal com $R e=0.026$ e $E r=5$ e condição de contorno $\phi=-90^{\circ}$. (a) Pressão no canal de entrada para $y=5.55$ e $0 \leq x \leq 6$. (b) Pressão no canal de saída para $x=5.55$ e $0 \leq y \leq 6 \ldots \ldots \ldots$

4.4 Variação das velocidades no L-canal com $R e=0.026$ e $E r=5$ e condição de contorno $\phi=-90^{\circ}$. (a) Velocidade no canal de entrada para $x=4.55 \mathrm{e}$ $5 \leq y \leq 6$. (b) Velocidade no canal de saída para $y=4.55$ e $5 \leq x \leq 6$. . . . .

4.5 Simulação numérica do diretor através de um L-canal com $R e=0.026$ e $E r=5$. Distribuição do diretor para diferentes condições de contorno para o ângulo $\phi$ : (a) $\phi=-90^{\circ}$, (b) $\phi=0$, e (c) $\phi=90^{\circ}$.

4.6 Variação do ângulo do diretor no L-canal com $R e=0.026$ e $E r=5$ e condição de contorno $\phi=-90^{\circ}$. (a) $y=5.55$ e $0 \leq x \leq 6$; (b) $x=5.55$ e $0 \leq y \leq 6$. 
4.7 Variação do ângulo do diretor no L-canal com $R e=0.026$ e $E r=5$ e condição de contorno $\phi=0^{\circ}$. (a) $y=5.55$ e $0 \leq x \leq 6$; (b) $x=5.55$ e $0 \leq y \leq 6$. . . . .

4.8 Variação do ângulo do diretor no L-canal com $R e=0.026$ e $E r=5$ e condição de contorno $\phi=90^{\circ}$. (a) $y=5.55$ e $0 \leq x \leq 6$; (b) $x=5.55$ e $0 \leq y \leq 6$. . . . .

4.9 Gráficos das linhas de corrente para $R e=0.026$ e $E r=5$ : (a) $\left(\phi=-90^{\circ}\right)$, (b) $(\phi=0)$ e $(\mathrm{c})\left(\phi=90^{\circ}\right)$.

4.10 Distribuição do diretor no L-canal para $R e=0.026$ e $R 2=0.4$ e diferentes números de Ericksen: (a) $E r=10$, (b) $E r=50$, (c) $E r=100$, (d) $E r=150$, (e) $E r=200$, e (f) $E r=250$.

4.11 Gráficos das linhas de corrente para $R e=0.026$ e $R 2=0.4$ e diferentes números de Ericksen: (a) $E r=10$, (b) $E r=50$, (c) $E r=100$, (d) $E r=150$, (e) $E r=200$, e (f) $E r=250$.

4.12 Distribuição do diretor no L-canal para $R e=0.026$ e $R 2=0.3$ e vários números de Ericksen: (a) $E r=10$, (b) $E r=50$, (c) $E r=100$, (d) $E r=150$, (e) $E r=200$, e (f) $E r=250$.

4.13 Gráficos das linhas de corrente para $R e=0.026$ e $R 3=0.3$ e diferentes números de Ericksen: (a) $E r=10$, (b) $E r=50$, (c) $E r=100$, (d) $E r=150$, (e) $E r=200$, e (f) $E r=250$.

4.14 Descrição do domínio para a simulação do escoamento em uma contração 4:1. (a) As setas vermelhas representam as condições de contorno utilizadas para o cálculo do ângulo $\phi$ dado pela equação (3.36). (b) $X$ é o comprimento dos vórtices de canto (Lvortex).

4.15 Simulação numérica do escoamento através de uma contração com $R e=0.013$ e $E r=10$. Isolinhas: (a) pressão, (b) componente $u$ e (c) componente $v$ da velocidade. .

4.16 Variação da pressão e do gradiente de pressão através de uma contração com $R e=0.013$ e $E r=10$. (a) pressão, (b) gradiente de pressão.

4.17 Perfis das velocidades $u$ e $v$ em diferentes seções do canal com $R e=0.013 \mathrm{e}$ $E r=10$. (a) $x=10.1$, (b) $x=19.9$ e (c) $x=30.1$.

4.18 Simulação através de uma contração com $R e=0.013$ e $E r=10$. (a) Variação do ângulo do diretor $\phi$ na direção $x$ para $y_{c}=4.1$. (b) Comparação das soluções numérica e analítica do ângulo do diretor $\phi$ para $x=30.1$.

4.19 Simulação numérica do diretor através de uma contração planar 4:1 com $R e=$ 0.013. Gráficos do diretor para diferentes números de Ericksen: (a) $E r=10$, (b) $E r=50$, (c) $E r=70$, (d) $E r=100$, (e) $E r=150$ e (f) $E r=200 \ldots \ldots$. . . . 100

4.20 Gráficos das linhas de corrente para $R e=0.013$ para diferentes números de Ericksen: (a) $E r=10$, (b) $E r=50$, (c) $E r=70$, (d) $E r=100$, (e) $E r=150 \mathrm{e}$ (f) $\operatorname{Er}=200$. 
4.21 Simulação numérica do diretor através de uma contração planar 4:1 com $R e=$ 0.026. Gráficos do diretor para diferentes números de Ericksen: (a) $E r=10$, (b) $E r=50$, (c) $E r=70$, (d) $E r=100$, (e) $E r=150$ e (f) $E r=200 \ldots \ldots 2$

4.22 Gráficos das linhas de corrente para $R e=0.026$ para diferentes números de Ericksen: (a) $E r=10$, (b) $E r=50$, (c) $E r=70$, (d) $E r=100$, (e) $E r=150$ e (f) $E r=200$.

4.23 Simulação numérica do diretor através de uma contração planar 4:1 com $R e=$ 0.066. Gráficos do diretor para diferentes números de Ericksen: (a) $E r=10$, (b) $E r=50$, (c) $E r=70$, (d) $E r=100$, (e) $E r=150$ e (f) $E r=200 \ldots \ldots$. . . . 104

4.24 Gráficos das linhas de corrente para $R e=0.066$ para diferentes números de Ericksen: (a) $E r=10$, (b) $E r=50$, (c) $E r=70$, (d) $E r=100$, (e) $E r=150 \mathrm{e}$ (f) $E r=200$.

4.25 Variação do comprimento do vórtice (Lvortex $=X / L$ ver Figura 4.14) em função do número de Ericksen. . . . . . . . . . . . . . . . . . . . . . . 106

4.26 Descrição do domínio computacional para a simulação do escoamento em uma expansão planar 1:4. (a) As setas vermelhas representam as condições de contorno utilizadas para o cálculo do ângulo $\phi$ dado pela equação (3.36). (b) $X$ representa o comprimento dos vórtices de canto (Lvortex).

4.27 Simulação Numérica do escoamento em uma expansão planar 4:1 com $R e=0.013$ e $E r=10$. Isolinhas: (a) velocidade $u$, (b) velocidade $v$, (c) pressão. . . . . . . . 109

4.28 Variação da pressão e do gradiente de pressão na linha central do canal com $R e=0.013$ e $E r=10$. (a) pressão, (b) gradiente de pressão. . . . . . . . . . . . 110

4.29 Perfis das velocidades $u$ e $v$ em diferentes seções do canal com $R e=0.013 \mathrm{e}$ $E r=10$. (a) $x=10.1$, (b) $x=20.1$ e (c) $x=30.1$.

4.30 Simulação numérica do diretor através de uma expansão planar 4:1 com $R e=$ 0.013. Gráficos do diretor para diferentes números de Ericksen: (a) $\operatorname{Er}=10$, (b) $E r=20,(\mathrm{c}) E r=50,(\mathrm{~d}) E r=100,(\mathrm{e}) E r=200,(\mathrm{f}) E r=500$ e (g) $E r=1000.112$

4.31 Gráficos das linhas de corrente para $R e=0.013$ e diferentes números de Ericksen: (a) $E r=10,(b) E r=50,(c) E r=100$, (d) $E r=200,($ e) $E r=500$ e (f) $\operatorname{Er}=1000$.

4.32 Gráficos das linhas de corrente para $R e=0.026$ e diferentes números de Ericksen: (a) $E r=10,(\mathrm{~b}) E r=50,(\mathrm{c}) E r=100$, (d) $E r=200$, (e) $E r=500$ e (f) $E r=1000$.

4.33 Simulação numérica do diretor através de uma expansão planar 4:1 com $R e=$ 0.028. Gráficos do diretor para diferentes números de Ericksen: (a) $E r=10$, (b) $E r=20$ (c) $E r=50,(d) E r=100,($ e) $E r=200$, (f) $E r=500$ e (g) $E r=1000.115$ 
4.34 Gráficos das linhas de corrente para $R e=0.028$ e diferentes números de Ericksen:

(a) $E r=10,(\mathrm{~b}) E r=50$, (c) $E r=100$, (d) $E r=200$, (e) $E r=500$ e (f)

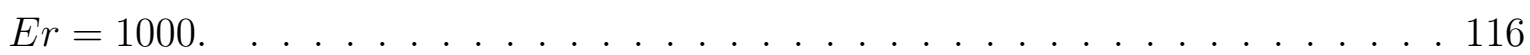

4.35 Gráficos das linhas de corrente para $R e=0.055$ e diferentes números de Ericksen:

(a) $E r=10,($ b) $E r=50$, (c) $E r=100$, (d) $E r=200$, (e) $E r=500$ e (f)

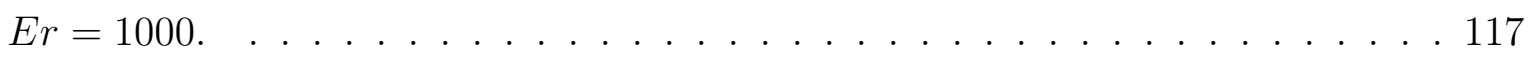

4.36 Gráficos da variação do comprimento do vórtice em função do número de Ericksen: (a) cristal líquido nemático $\mathrm{MBBA}$ à $25^{\circ} \mathrm{C}$, (b) cristal líquido nemático MBBA à $42^{\circ} \mathrm{C}$, (c) escala de velocidade utilizada $U_{1}=0.001 \mathrm{~ms}^{-1}$ e (d) escala de velocidade utilizada $U_{2}=0.002 \mathrm{~ms}^{-1} \ldots \ldots \ldots$. . . . . . . . . 118 


\section{Lista de Tabelas}

2.1 Erro relativo na velocidade $v$ nas malhas $\mathbf{M 1}$, M2, M3 e ordem de convergência. 46

2.2 Erro relativo na velocidade $w$ nas malhas $\mathbf{M 1}, \mathbf{M} 2, \mathbf{M} 3$ e ordem de convergência. 46

3.1 Erros Relativos obtido nas malhas $\mathrm{M}_{0}, \mathrm{M}_{1}$ e $\mathrm{M}_{2} \ldots \ldots \ldots \ldots$

4.1 Comprimento dos vórtices em função do número de Ericksen. . . . . . . . . . . . 105

4.2 Comprimento do vórtice de canto (LVortex) em função do número de Ericksen para o cristal líquido nemático - MBBBA à 25․ . . . . . . . . . . . . . 111

4.3 Comprimento do vórtice de canto (LVortex) em função do número de Ericksen para o cristal líquido nemático - MBBA à $42^{\circ} \ldots$. . . . . . . . . . . . . . 111

A.1 Fatores de conversão para grandezas[72, 95]. Esta tabela permite simples conversões das unidades cgs Gaussianas para unidades no sistema SI. As abreviações usadas foram: (P)poise, (G) gauss, (Oe) oersted, (N) newton, (Pa s) pascal segundo, $(\mathrm{T})$ tesla,$(\mathrm{A})$ ampère, $(\mathrm{V})$ volt, $(\mathrm{J})$ joule. $c \doteqdot 2.998 \times 10^{8} \mathrm{~m} \mathrm{~s}^{-1}$ representa o valor numérico da velocidade da luz no sistema SI. . . . . . . . . . . . . 135

A.2 Valores de parâmetros físicos para as fases nemáticas de MBBA, PAA e 5CB no sistema de unidades SI; os valores de $\chi_{a}$ também são mostrados no sistema de unidades cgs. A temperatura de transição de nemático para isotrópico é denotada por $T_{N I}$. As viscosidades para $5 \mathrm{CB}$ foram deduzidas de uma tabela fornecida por [76] e [75] utilizando fórmulas de conversão padronizadas [123]. 136

A.3 Constantes Fundamentais. As abreviações utilizadas são: henry (H), farad (F),

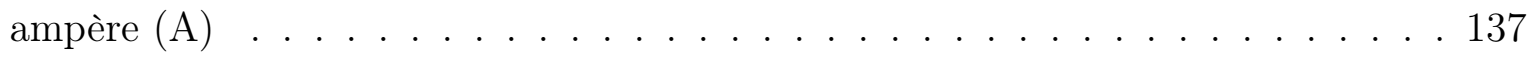




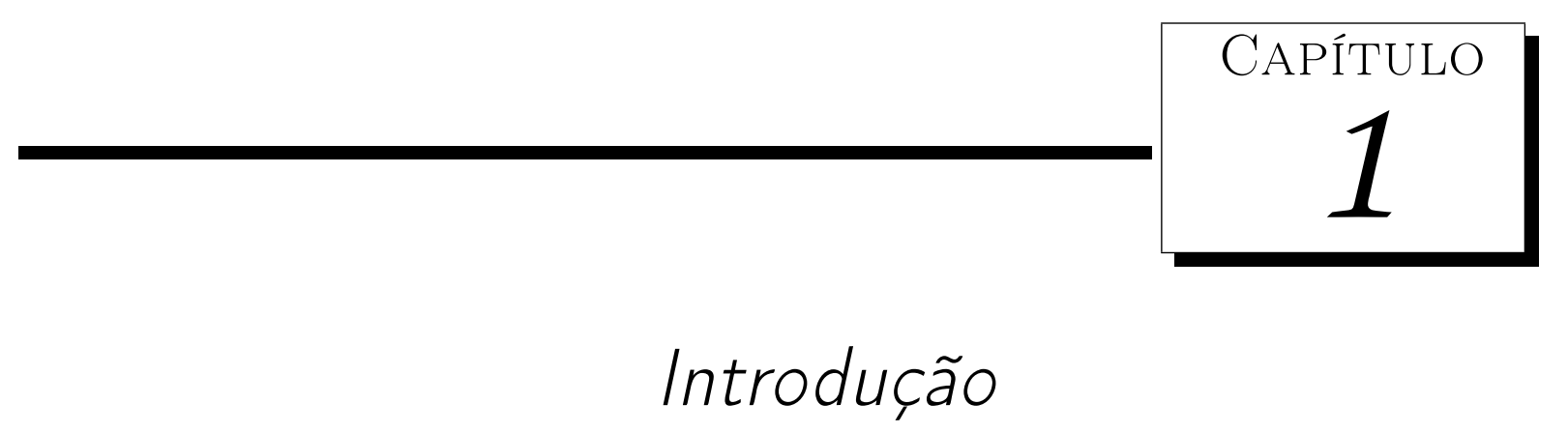

Nesse capítulo é apresentado alguns conceitos sobre cristais líquidos, uma descrição da teoria dos cristais líquidos e o desenvolvimento da teoria dinâmica dos cristais líquidos nemáticos. Kelker [74] escreveu um trabalho complexo descrevendo a história e desenvolvimento dos cristais líquidos. Os livros de Collings e Hird [30] e de Jeu [39] fornecem introduções técnicas sobre a física e estrutura dos cristais líquidos enquanto relatos mais avançados da teoria e aplicações dos cristais líquidos podem ser encontrados nos livros de Prost e de Gennes [37, 38], Chandrasekhar [23] e Blinov [12], assim como nas revisões de Stephen e Straley [120] e Leslie [83] e no livro recente de Stewart [123].

\subsection{O que é um Cristal Líquido?}

Em geral, na física dos materiais, é aceito que existam três estados da matéria: sólido, líquido e gasoso [79]. As substâncias podem exibir todas estas fases à medida que a temperatura é variada. Por exemplo, na condição de pressão atmosférica, a água é sólida a uma temperatura abaixo de $0^{\circ} \mathrm{C}$, líquida para temperaturas entre $0^{\circ} \mathrm{C}$ e $100^{\circ} \mathrm{C}$ e gasosa se a temperatura estiver acima de $100^{\circ} \mathrm{C}$. Entretanto, esta simples descrição não é exata para muitos materiais. Alguns sólidos, quando aquecidos, fundem-se formando um líquido leitoso a uma certa temperatura, e a uma temperatura mais alta esse líquido torna-se transparente (por exemplo benzoato de colesterila). Esses materiais, quando são aquecidos, em vez de passar diretamente da fase sólida para a fase líquida, passam por uma fase intermediária denominada fase de cristal líquido [80]. Nesta fase, esses materiais apresentam a mesma estrutura dos sólidos e a mesma liberdade de movimento possuída pelos líquidos. Os cristais líquidos são, como o nome sugere, materiais que 
podem existir entre a fase sólida e a fase líquida isotrópica ${ }^{1}$. Esses materiais são conhecidos por serem anisotrópicos $[29,30,39]$, porque exibem diferentes propriedades físicas em diferentes direções.

Um dos conceitos fundamentais presentes na discussão do termo cristal líquido é o ordenamento das moléculas. Em um sólido as moléculas estão organizadas espacialmente em posições bem definidas e dizemos que essas moléculas apresentam ordem posicional de longo alcance. Por outro lado, em um líquido isotrópico suas moléculas não possuem ordem posicional. Entretanto, a ordem posicional não é o único ordenamento existente na natureza que pode se manifestar na estrutura da matéria. A ordem orientacional das moléculas da matéria traz consigo novas possibilidades de organização molecular em diferentes escalas de comprimento. Como exemplo, vamos considerar uma substância constituída de moléculas com a forma de um bastão e que os centros de massa dessas moléculas não apresentam nenhum tipo de ordem posicional de longo alcance como nos sólidos. Vamos ainda supor que exista um ordenamento dessas moléculas: os eixos maiores das moléculas estão orientados segundo uma determinada direção preferencial no espaço. Logo, há um ordenamento orientacional de longo alcance. Em outras palavras, se conhecermos a orientação preferencial de uma das moléculas podemos afirmar que a outra molécula, bem distante daquela sobre a qual conhecemos a posição e orientação do eixo maior, estará também com seu eixo maior orientado segundo aquela direção preferencial (ver Figura 1.1). Entretanto, não podemos dizer com certeza onde estará o seu centro de massa, como poderíamos afirmar caso se tratasse de um sólido. Assim temos, portanto, propriedades de um líquido isotrópico com algum grau de ordem e com orientação de longo alcance. A Figura 1.1 ilustra um esboço das fases cristal sólido, cristal líquido, líquido isotrópico e gasoso com as moléculas em forma de bastão.

Com relação a Figura 1.1, pode-se notar que na fase sólida as moléculas encontram-se ordenadas com posição e orientação de longo alcance. O mesmo não acontece para as moléculas na fase líquida isotrópica.

A maioria dos cristais líquidos são materiais orgânicos, e se dividem em duas grandes categorias, de acordo com os parâmetros mais relevantes nas transições de fase; os termotrópicos obtidos pela variação de temperatura, e os liotrópicos que podem ser obtidos através da variação da concentração dos componentes em um dado solvente.

Os cristais líquidos termotrópicos podem ainda serem analisados e estudados sob o ponto de vista da massa molar. Desta forma, os cristais líquidos são divididos em dois grupos, cristais líquidos não poliméricos e cristais líquidos poliméricos. A combinação de características específicas de polímeros, tais como, propriedades mecânicas, elétricas, ópticas e térmicas combinadas com a anisotropia e birrefringência, as quais são características físico-químicas dos cristais líquidos permitem a obtenção de novos materiais com propriedades físico-químicas diferencia-

\footnotetext{
${ }^{1}$ Uma substância é isotrópica, quando suas propriedades físicas são as mesmas em todas as direções; por exemplo, a água é isotrópica à temperatura ambiente.
} 


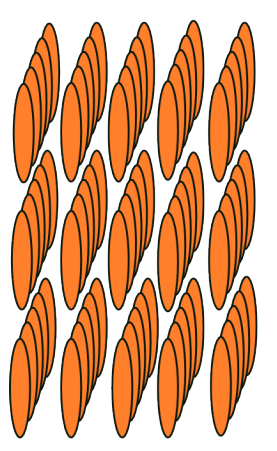

Fase sólida

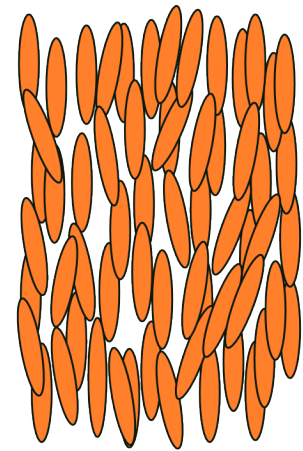

Fase cristal líquido

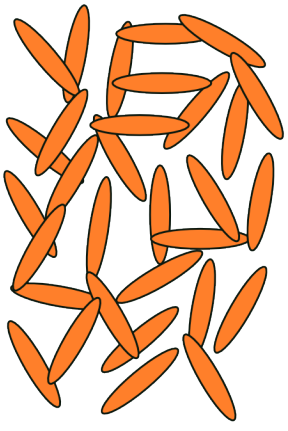

Fase líquida isotrópica

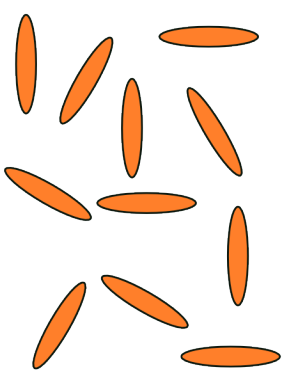

Fase gasosa

Temperatura

Figura 1.1: Transição de fase devido ao aumento de temperatura dos estados da matéria: sólido cristalino, cristal líquido, líquido isotrópico e gás.

das. Na qual, cada tipo de estrutura dentro de um faixa de temperatura específica apresenta fase líquida-cristalina e as propriedades dos polímeros. Neste trabalho será investigado apenas cristais líquidos nemáticos (termotrópicos) não poliméricos.

\subsection{A origem dos cristais líquidos}

A descoberta dos cristais líquidos é atribuída a Frederick Reinitzer [107] (uma tradução em inglês deste artigo está disponível em [108]), um botânico austríaco, cujo principal interesse era investigar a função do colesterol nas plantas. Em 1888, Reinitzer descobriu que uma mistura orgânica que estava estudando, posteriormente chamada de benzoato de colesterila, tinha dois pontos distintos de fusão. Em seus experimentos, Reinitzer aumentou a temperatura de uma amostra sólida, e observou que esta amostra transitou para um líquido turvo e aumentando ainda mais a temperatura, o material transitou novamente para um líquido transparente. $\mathrm{Na}$ mesma época, um físico alemão chamado Otto Lehmann, observou que algumas substâncias sólidas fundiam, passando por um estado intermediário no qual o líquido era birrefringente (propriedades ópticas). Reinitzer contatou Otto Lehmann detalhando sua descoberta e solicitando mais análises de sua amostra. Em 1889 Lehmann [80] usou o termo "cristais que escoam" para descrever o material das amostras, e por volta de 1900 ele designou o termo "cristal líquido". Nos anos seguintes, Lehmann observou que um cristal líquido em contato com uma superfície sólida reorientava-se à uma certa direção. Esta observação foi de grande importância quando as primeiras aplicações dos cristais líquidos em mostradores foram consideradas.

Em 1922, baseado nas diferentes ordenações moleculares de cada substância, Friedel [60] descreveu e propôs a classificação das diferentes fases dos cristais líquidos em três categorias que ele chamou nematic (palavra grega $\nu \eta \mu \alpha$ que significa "linha"), smectic (palavra grega $\sigma \mu \eta \gamma \mu \alpha$ que significa "sabão") e cholesteric, terminologia que está agora em comum uso. Friedel também 
observou que os cristais líquidos podem ser orientados por um campo elétrico. O efeito de campos elétricos e magnéticos em cristais líquidos tornou-se o foco de grande interesse de muitos pesquisadores $[14,15,59,115]$. Uma base matemática para o estudo de cristais líquidos foi desenvolvida por Oseen [101, 102] na Suécia, que estudou as propriedades elásticas dos cristais líquidos. Utilizando as ideias de Oseen, Frank [57] estabeleceu a teoria estática dos cristais líquidos em 1958 e em 1960 Ericksen apresentou a teoria para fluidos anisotrópicos baseado em um vetor unitário, chamado diretor, que representa o eixo anisotrópico das moléculas [45]. Logo após, em 1961, Ericksen [46] generalizou a teoria estática dos cristais líquidos propondo leis de balanço para o comportamento dinâmico dos cristais líquidos. Usando estas ideias, Leslie [81, 82] publicou dois artigos onde desenvolveu a teoria dinâmica para cristais líquidos, que levou a criação da teoria dinâmica de Ericksen-Leslie para cristais líquidos nemáticos.

Em 1968 cientistas da RCA (Radio Corporation of America) demonstraram que uma fina camada de cristal líquido alterou da cor opaca para a cor clara quando um campo elétrico foi aplicado no cristal liquido. Este foi o primeiro mostrador de cristal líquido (LCD) desenvolvido [29]. Na década de 70, Gray e seus colaboradores [63, 64] sintetizaram um cristal líquido nemático que ficou estável à temperatura ambiente. Anos depois mostradores de cristal líquido foram criados e usados na produção de equipamentos operados com bateria tais como televisores e calculadoras. Hoje em dia cristais líquidos estão presentes em muitas formas. LCDs para computadores, celulares, câmeras, calculadoras, TVs, etc. Outra importante aplicação dos cristais líquidos está nos termômetros, pois, sabe-se que alguns cristais líquidos mudam de cor com mudança de temperatura. Utilizando essa propriedade, importantes e práticas aplicações tem sido desenvolvidas em diversas áreas como medicina e eletrônica. Por exemplo, aparelhos de cristais líquidos podem ser fixados na pele para mostrar um mapa das temperaturas, isto pode ser útil devido aos frequentes problemas médicos, tal como um tumor cancerígeno, que tem uma temperatura diferente do tecido vizinho. É certo que o entendimento dos cristais líquidos continuará se desenvolvendo através dos anos com novas descobertas e aplicações.

A área de pesquisa em cristais líquidos é um campo recente da ciência que envolve cientistas de várias disciplinas, tais como química, física, engenharia, ciência dos materiais, biologia, matemática e ciência da computação.

\subsection{Aplicações dos Cristais Líquidos}

Cristais líquidos são conhecidos desde os trabalhos de Reinitzer [107] e Lehmann [80] a mais de 100 anos atrás e suas propriedades eletro ópticas foram investigadas nos primeiros estudos de Oseen [101] em 1929, Freedericksz [59] em 1933, e por muitos outros pesquisadores. Entretanto, o interesse nas aplicações industriais de cristais líquidos só surgiu no final da década de 60.

Devido a organização molecular, os cristais líquidos nemáticos são muito sensíveis a ação de campos externos tais como campos elétricos e magnéticos. Isso faz dos cristais líquidos nemáticos ótimos para aplicações industriais. Os cristais líquidos estão virtualmente em toda 
parte. Eles ficaram mais conhecidos da população através da sigla LCD (Liquid Crystal DisplayMostrador de Cristal Líquido). O primeiro mostrador utilizado industrialmente foi o mostrador de cristal líquido nemático torcido ("TN-LCD = twisted nematic liquid crystal display"). Este mostrador utiliza um cristal líquido nemático intercalado entre duas placas de vidro espaçadas de $5-10 \mu \mathrm{m}$ de distância. O cristal líquido é introduzido entre as duas placas de vidro através de ação capilar e/ou através de uma diferença de pressão aplicada. As placas de vidro são esfregadas de modo a provocar uma direção preferencial, em que as moléculas nemáticas se alinham paralelas a essa direção. A direção de esfregamento das placas de vidro é paralela à direção local de polarização, induzindo uma torção de $90^{\circ}$ do topo para o fundo da célula. Em virtude da anisotropia óptica do cristal líquido, a direção de polarização da luz na célula é movida seguindo a orientação do diretor. Como, resultado a luz é transmitida pelo analisador. Isso corresponde a uma célula clara, veja Figura 1.2(a).

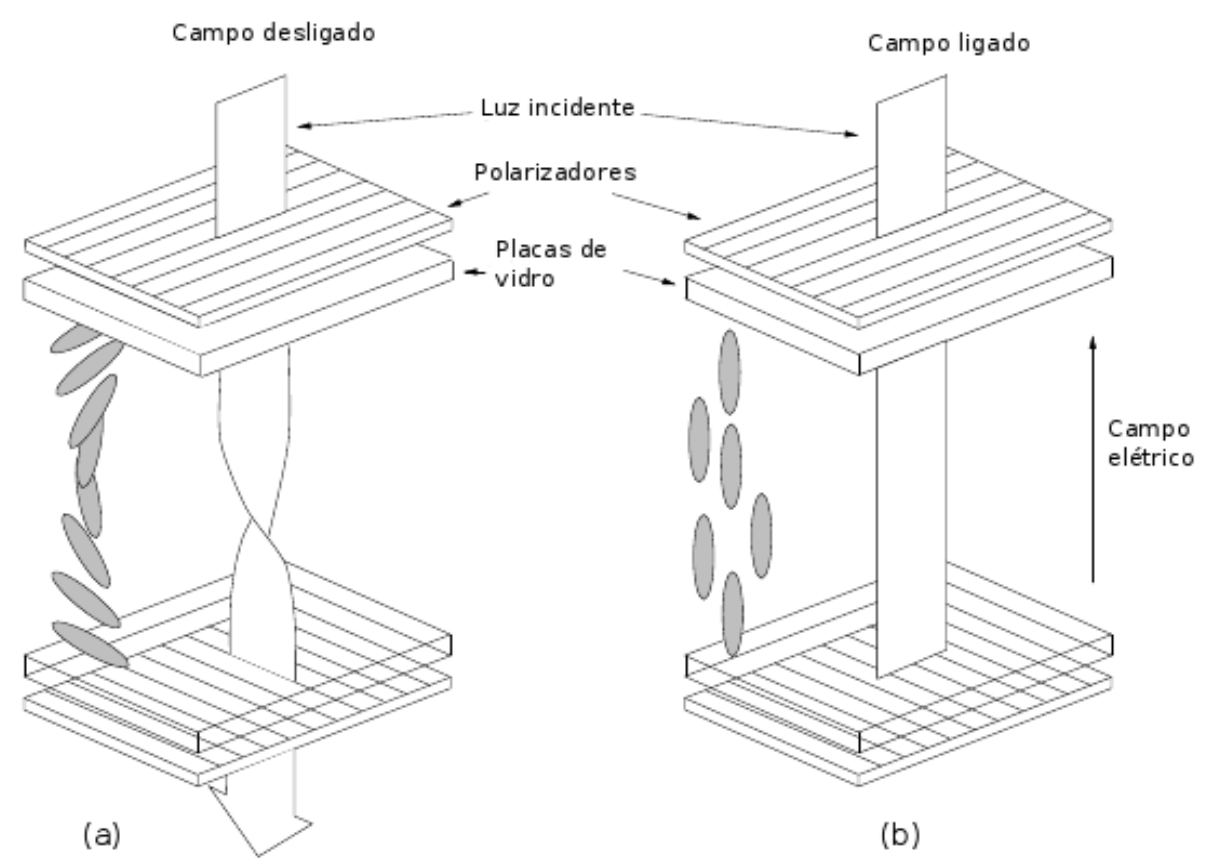

Figura 1.2: Mostrador de cristal liquido torcido. Essa figura foi extraída do trabalho de Mottram e Hogan [96].

Com a aplicação de um campo elétrico entre as placas, as moléculas se reorientam para ficar aproximadamente paralelas ao campo elétrico. Isso corresponde ao estado escuro da célula, veja Figura 1.2(b). Quando o campo elétrico é removido as moléculas voltam ao seu estado inicial, ou seja, com a estrutura torcida (ver Figura 1.2(a)) e então a célula aparecerá clara novamente. O display de cristal líquido nemático torcido (TN) é utilizado em alguns tipos de relógio de pulso [62], calculadoras, etc. Para satisfazer as exigências de mostradores mais sofisticados, 
como esses encontrados em telefones celulares ou laptops, foi projetado um mostrador mais avançado. Um exemplo é o mostrador de cristal líquido nemático super torcido (STN). Trata-se de um aperfeiçoamento direto do mostrador de TN, que permite uma torção de $270^{\circ}$ em vez de $90^{\circ}$. Isso resulta em uma transição mais precisa e mais rápida entre os estados claros e escuros. Atualmente, os LCDs são utilizados em telas de computadores, jogos eletrônicos, câmeras fotográficas e de vídeo. Em resumo, a vantagem da utilização de cristais líquidos em mostradores está no seu baixo consumo de energia, durabilidade, definição de imagem e pequeno tamanho.

Há outras inúmeras aplicações para os cristais líquidos em diferentes áreas. Por exemplo cristais líquidos têm sido propostos para aplicações na Engenharia [44], em que a variação dos coeficientes de viscosidade em diferentes fases pode conduzir ao desenvolvimento de lubrificantes bastante eficientes. Recentemente, pesquisadores do Instituto Fraunhofer [58], na Alemanha, testaram cristais líquidos como lubrificantes. Nos primeiros testes, o lubrificante de cristal líquido fez o atrito entre duas placas metálicas cair quase a zero, com um rendimento que supera em larga margem os melhores óleos lubrificantes existentes atualmente. Agora os pesquisadores esperam resolver a questão econômica para a disseminação do uso do novo lubrificante, pois cristais líquidos são produzidos para o mercado de telas e monitores e são muito caros.

Outras aplicações para os cristais líquidos se dão através dos cristais líquidos poliméricos (LCPs) [111, 143], importantes para processamento de polímeros de peças eletrônicas devido à suas propriedades mecânicas tais como resistência e rigidez, maleabilidade e alta resistência térmica. As características dos produtos moldados de cristais líquidos poliméricos resultam da orientação molecular no interior do produto.

Uma outra aplicação de cristais líquidos poliméricos que tem sido desenvolvido com sucesso para a indústria é a área de fibras de alta resistência. Kevlar (marca registrada da DuPont), que é utilizado para produzir vários produtos como capacetes, cintos de segurança, alguns modelos de raquetes de tênis e coletes à prova de bala, é apenas um exemplo do uso de cristais líquidos poliméricos em aplicações dos chamados materiais de baixo peso porém muito resistentes.

\subsection{Conceitos Introdutórios da Teoria dos Cristais Líqui- dos}

Muitos cristais líquidos são essencialmente constituídos de moléculas alongadas em forma de bastão (embora existam outros tipos de estruturas onde as moléculas tem a forma de um disco) onde a direção média do alinhamento molecular no espaço é representada pelo vetor unitário $\mathbf{n}$, comumente chamado diretor [123] (ver Figura 1.3).

Esses materiais não possuem polaridade e portanto as duas possíveis orientações do diretor são equivalentes, ou seja, n é equivalente a -n. Embora o diretor possa, em geral, apontar em qualquer direção, existem muitos casos onde esta direção é influenciada por forças externas. Por exemplo, quando o cristal líquido esta em contato com um contorno sólido o diretor tende a 


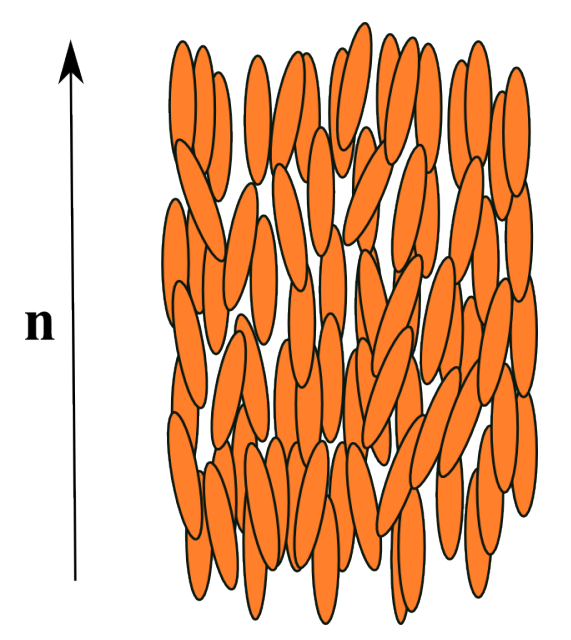

Figura 1.3: A direção média das moléculas é representada pelo vetor unitário conhecido como diretor.

alinhar-se paralelo a esta superfície. Também, campos elétricos e magnéticos podem direcionar o diretor a um ângulo particular, o que permite a manipulação de cristais líquidos.

Existem diferentes fases de cristal líquido, e cada uma dessas fases tem a sua própria característica [123]. Por exemplo, na fase nemática as moléculas tendem a alinhar-se paralelas umas as outras ao longo de uma direção preferencial determinada pelo diretor $\mathbf{n}$. Nos cristais líquidos nemáticos, as moléculas não possuem ordem posicional de longo alcance. Entretanto, há um ordenamento orientacional de longo alcance dessas moléculas como pode ser visto na Figura 1.3. Outra fase encontrada nos cristais líquidos é a fase esmética. A Figura 1.4a) ilustra a estrutura da fase esmética $\mathrm{A}$, em que as moléculas do material estão organizadas em camadas e o diretor é perpendicular as camadas. A Figura 1.4b) representa a estrutura da fase esmética C. Nessa fase, além das moléculas se organizarem em camadas, como na fase esmética A, os eixos maiores das moléculas estão alinhados, em média, a um ângulo $a$ em relação à direção normal às camadas. Estes são apenas alguns exemplos das fases de cristais líquidos; há outras fases ainda mais complexas existentes nos cristais líquidos.

Nesta tese estamos somente interessados nos cristais líquidos nemáticos, que é a fase mais simples e umas das fases mais comuns dos cristais líquidos. 

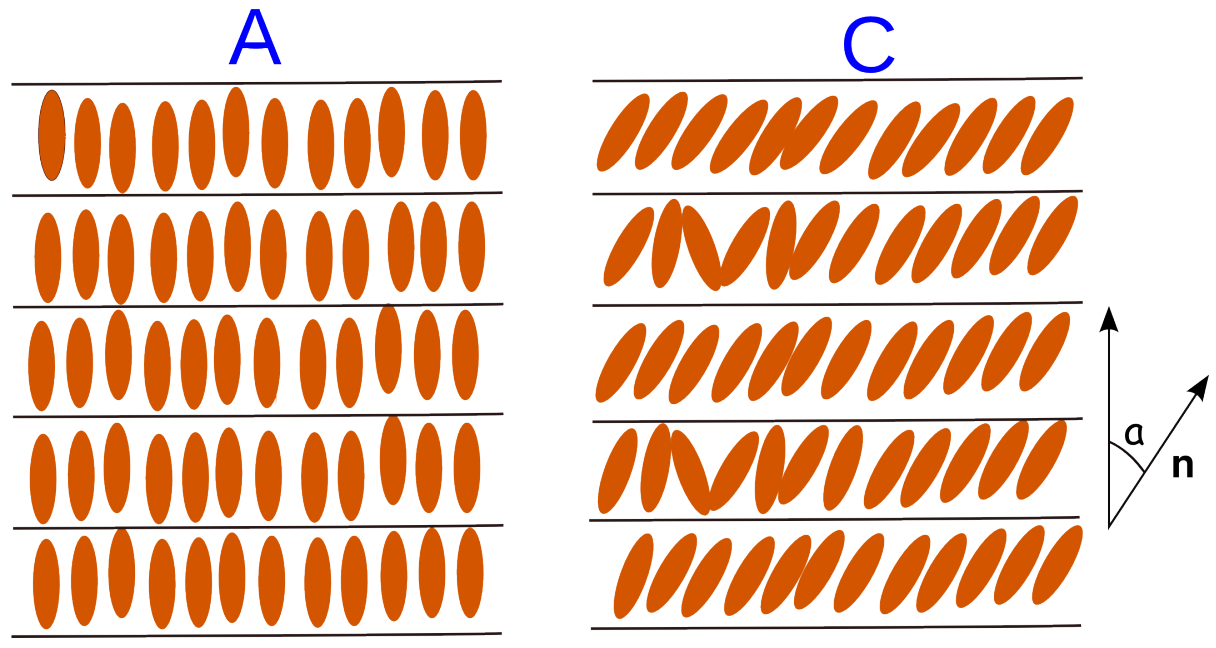

Figura 1.4: Representação das fases esmética A e esmética C.

\subsubsection{Elasticidade}

Em termos físicos, um material é classificado como elástico se o mesmo deforma sob tensão e retorna a sua forma original quando a tensão é removida. O cristais líquidos são materiais que possuem propriedades elásticas. A elasticidade é uma propriedade comum entre os cristais líquidos e os sólidos.

As deformações em cristais líquidos podem ser descritas como uma combinação de três tipos básicos de deformação:

- Afunilamento (SPLAY): essa deformação é obtida quando tem-se duas placas que formam um ângulo $\phi \neq 0$ e as moléculas próximas às placas estão paralelas com às mesmas (ver Figura 1.5(a));

- Torção (TWIST): este tipo de deformação ocorre quando as moléculas vizinhas são rotacionadas para fora do plano do diretor (ver Figura 1.5(b));

- Flexão (BEND): essa deformação ocorre quando tem-se duas placas que formam um ângulo $\phi \neq 0$ e as moléculas próximas as placas estão perpendiculares às mesmas (ver Figura 1.5(c)).

Essas deformações geram uma energia elástica que está relacionada com as constantes elásticas de Frank [57] $K_{1}$ (splay), $K_{2}$ (twist) e $K_{3}$ (bend), respectivamente. Uma interpretação matemática mais detalhada dessas constantes pode ser encontrado em Virga [138]. 
(a) splay

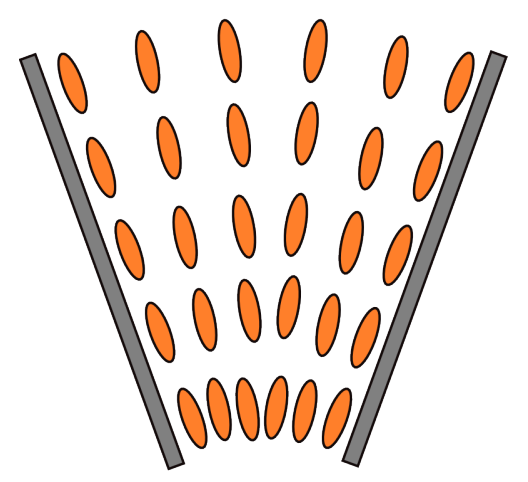

(b) twist

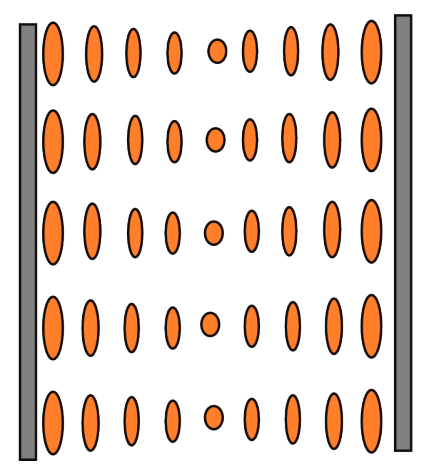

(c) bend

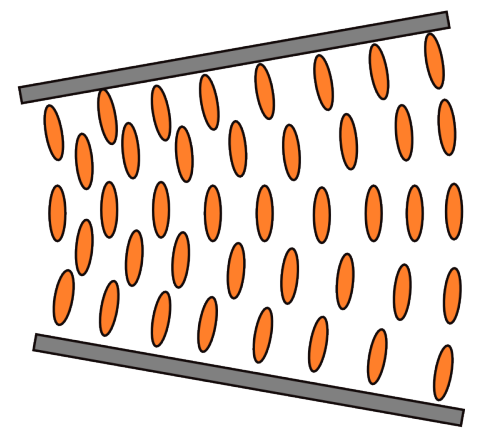

Figura 1.5: Tipos de deformações elásticas básicas: (a) afunilamento (splay) $\left(K_{1}\right)$, (b) torção (twist) $\left(K_{2}\right)$ e (c) flexão (bend) $\left(K_{3}\right)$.

\subsubsection{Viscosidades de Miesowicz}

Devido a natureza fluídica dos cristais líquidos, o movimento das moléculas esta associado a uma fricção interna ou viscosidade. Fluidos isotrópicos possuem somente um coeficiente de viscosidade que é definido em função da taxa de cisalhamento e pressão. Entretanto, os cristais líquidos nemáticos possuem seis coeficientes de viscosidade [48], denotados por $\alpha_{i}(i=1,2, \ldots, 6)$, que são conhecidos como as viscosidades de Leslie. Com exceção de $\alpha_{4}$, as viscosidades de Leslie, em geral, não podem ser identificadas individualmente; porém, certas combinações lineares dessas viscosidades podem ser identificadas experimentalmente. As viscosidades de Leslie podem ser representadas em termos das viscosidades de Miesowicz. A primeira determinação dessas viscosidades foi realizada por Miesowicz [89] na década de 30. Mais tarde em 1946, Miesowicz [90] distinguiu três principais viscosidades em um escoamento cisalhante $\eta_{1}, \eta_{2}$ e $\eta_{3}$ que podem ser medidas experimentalmente considerando a orientação do diretor $\mathbf{n}$ em relação a velocidade do escoamento u. Uma revisão histórica das viscosidades de Miesowicz e uma descrição detalhada do aparato mecânico utilizado em seus experimentos podem ser encontrados no trabalho de Miesowicz publicado em 1983 [91]. Mais informações sobre as viscosidades de Miesowicz podem ser encontrados nos artigos de revisão de Moscicki [94], Martins [87], Belyaev [11] e Schneider e Kneppe [116].

A Figura 1.6 apresenta uma ilustração das geometrias utilizadas por Miesowicz para o cálculo das viscosidades $\eta_{1}, \eta_{2}$ e $\eta_{3}$. Pode-se notar que $\eta_{3}$ está diretamente relacionada com a viscosidade de fluidos newtonianos. Existe uma quarta viscosidade de Miesowicz $\eta_{12}$ que não pode ser visualizada, mas está presente quando $\mathbf{n}$ não está alinhado ao longo $\mathbf{u}, \nabla \mathbf{u}$ ou $\mathbf{u} \times \nabla \mathbf{u}$. 
(a)

(b)

(c)

(d)
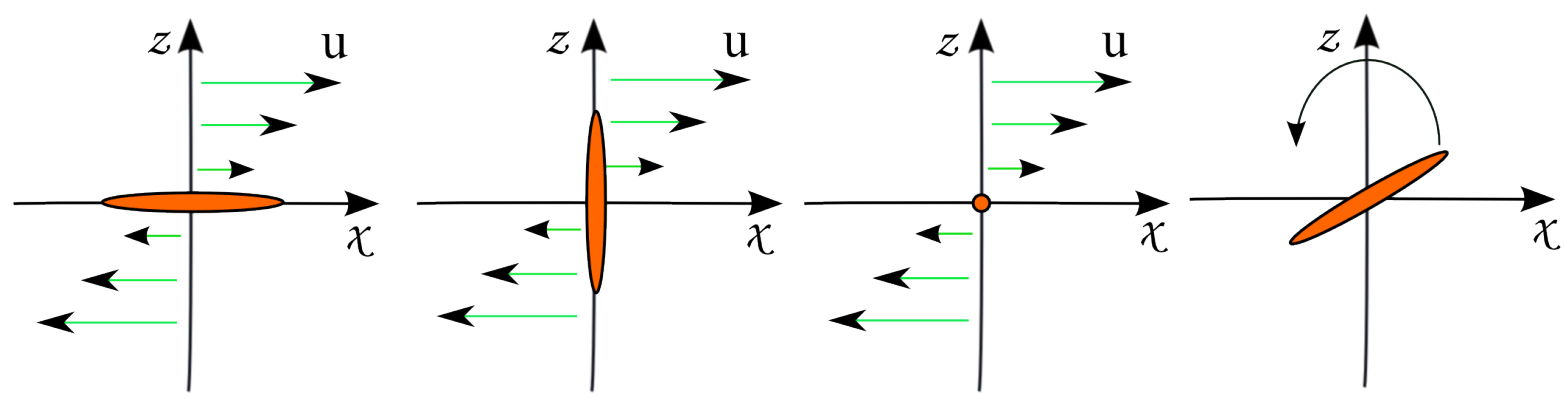

Figura 1.6: As viscosidades de Miesowicz: (a) viscosidade cisalhante $\eta_{1}(\mathbf{n} \| \mathbf{u}$ ), (b) viscosidade cisalhante $\eta_{2}(\mathbf{n} \| \nabla \mathbf{u}),(\mathrm{c})$ viscosidade cisalhante $\eta_{3}(\mathbf{n} \perp \mathbf{u}$ e $\mathbf{n} \perp \nabla \mathbf{u})$ e (d) viscosidade rotacional $\gamma_{1}$. A velocidade $\mathbf{u}$ é representada pelas setas. O diretor $\mathbf{n}$ é representado pelo bastão laranja.

\subsubsection{Ancoramento}

Na construção de LCDs (dispositivos eletro-ópticos) é necessário confinar a amostra de cristal líquido entre duas placas. As paredes dessas placas interagem com o cristal líquido, quebrando a simetria espacial e dessa forma alterando as características da camada superficial que se propagam para o volume, interferindo na orientação do diretor. Essa interação entre a placa e a camada superficial de cristal líquido é chamada de ancoramento. Em geral, a estrutura de um cristal líquido é facilmente influenciada por efeitos viscosos, elásticos, elétricos ou magnéticos. No entanto, próximo as placas, as forças superficiais podem ser muito fortes e assim impor uma orientação bem definida ao diretor quando o cristal liquido está em contato com as placas [119]. Esta situação é chamada de forte ancoramento. Dependendo como cada placa é preparada, é possível controlar a orientação do diretor. Por exemplo, quando uma superfície de vidro revestida com cristal liquido polimérico é esfregada em uma direção, o diretor tende a se alinhar ao longo dessa direção no plano da superfície [38].

(a) Orientação Planar

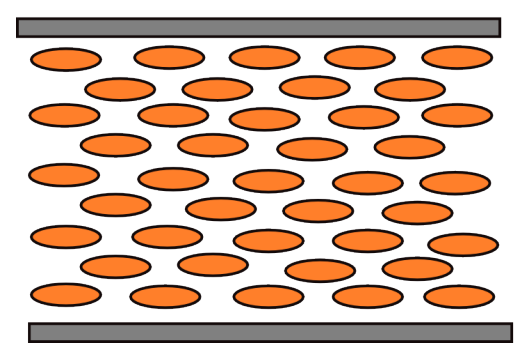

(b) Orientação Homeotrópica

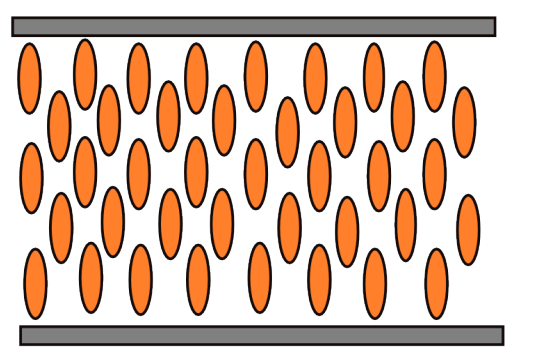

Figura 1.7: Dois possíveis tipos de forte ancoragem molecular: (a) Orientação Planar e (b) Orientação Homeotrópica. 
A Figura 1.7 ilustra dois tipos de ancoragem utilizados na prática. A orientação planar (ou homogênea) (Figura 1.7(a)), as moléculas encontram-se paralelas as placas. Na orientação homeotrópica (Figura 1.7(b)) o diretor é forçado a ficar perpendicular as placas.

Outros tipos de ancoragem nas placas são possíveis, como a fraca ancoragem [123]; entretanto, tais tipos de ancoragem não serão considerados nesta tese.

\subsection{Teoria Dinâmica dos Cristais Líquidos Nemáticos}

A teoria constitutiva para os cristais líquidos iniciou-se com os trabalhos de Ossen [100] em 1925, Zocher [146] em 1927 e Anzelius [5] em 1931. Ossen derivou uma versão estática da teoria dinâmica para os cristais líquidos nemáticos que foi de fundamental importância, especialmente quando a teoria estática foi reformulada por Frank [57] em 1958. A primeira teoria dinâmica amplamente aceita foi formulada por Ericksen [46] em 1961, em que, Ericksen generalizou a teoria estática para propor leis de balanço para o comportamento dinâmico dos cristais líquidos nemáticos. Esta teoria dinâmica foi então completada em 1968 por Leslie [81, 82], que seguindo as ideias de Ericksen, formulou equações constitutivas apropriadas e desse modo propôs expressões para várias contribuições dinâmicas. A teoria resultante conduziu à Teoria Dinâmica de Ericksen-Leslie para cristais líquidos nemáticos [123], que tem sido aplicada com sucesso para descrever muitas das características peculiares do comportamento dos cristais líquidos nemáticos.

Embora a teoria de Ericksen-Leslie seja bem sucedida e a mais utilizada na modelagem de escoamentos de cristais líquidos nemáticos, outras teorias são amplamente utilizadas. Por exemplo Ericksen [47] desenvolveu uma teoria que inclui a ordem do parâmetro do cristal líquido. Essa teoria foi considerada por Calderer e Liu [18] em um estudo de escoamentos com pressão controlada ("pressure-driven flows"). Outra formulação utilizada é a teoria Doi [41], que foi utilizada por Feng e Leal [49] para descrever como a geometria do canal influencia a orientação do cristal líquido durante o escoamento com pressão controlada. Estas teorias não serão discutidas neste trabalho; entretanto mais detalhes podem ser encontrados em [111, 112].

Na próxima seção, será apresentado resumidamente as equações dinâmicas de Ericksen-Leslie para cristais líquidos nemáticos. Uma derivação detalhada destas equações é apresentada por Stewart [123].

\subsubsection{O diretor}

Nas seções e capítulos seguintes desta tese será necessário uma definição matemática do diretor n. Uma das possíveis formas gerais do diretor é apresentada a seguir

$$
\mathbf{n}=(\cos \phi \cos \theta, \operatorname{sen} \phi \cos \theta, \operatorname{sen} \theta)
$$

onde os ângulos $\theta$ e $\phi$, mostrados na Figura 1.8, são denominados ângulo de inclinação e ângulo de torção, respectivamente. O ângulo $\theta$ representa a inclinação do diretor para fora do plano 
$x y$ enquanto o ângulo $\phi$ representa a torção do diretor sobre a normal ao plano. Note que nessa representação o diretor é automaticamente um vetor unitário.

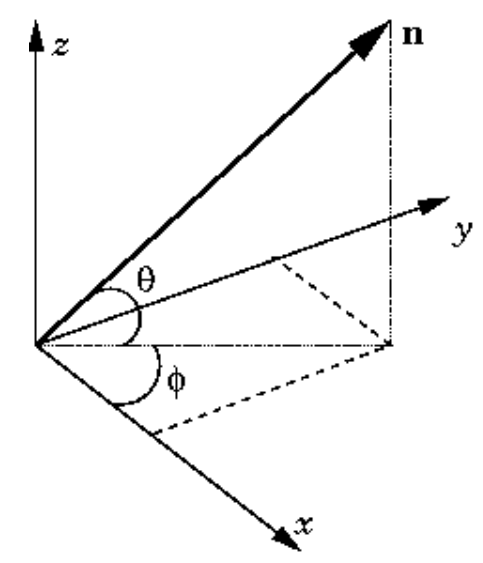

Figura 1.8: Representação espacial do diretor $\mathbf{n}$ em termos dos ângulos $\theta$ e $\phi$.

\subsubsection{Equações Dinâmicas de Ericksen-Leslie}

As equações dinâmicas de Ericksen-Leslie para cristais líquidos nemáticos isotérmicos incompressíveis quando o termo inercial do diretor é desprezado podem ser expressas como segue, usando a notação de Einstein onde apropriadas.

Essas equações consistem da restrição

$$
n_{i} n_{i}=1
$$

da equação de conservação de massa

$$
v_{i, i}=0
$$

junto com as leis de balanço que surgem das equações de momento linear e angular, ou seja,

$$
\begin{gathered}
\rho \dot{v}_{i}=\rho F_{i}-\left(p+w_{F}\right)_{, i}+\widetilde{g}_{j} n_{j, i}+G_{j} n_{j, i}+\widetilde{t}_{i j, j}, \\
\left(\frac{\partial w_{F}}{\partial n_{i, j}}\right)_{, j}-\frac{\partial w_{F}}{\partial n_{i}}+\widetilde{g}_{i}+G_{i}=\lambda n_{i},
\end{gathered}
$$

em que $\rho$ é a densidade, $F_{i}$ é a força externa por unidade de massa, $p$ é a pressão, $G_{i}$ é a força externa generalizada que está relacionada com o momento externo $\mathbf{K}$ por unidade de massa através da relação $\rho \mathbf{K}=\mathbf{n} \times \mathbf{G}$ e $w_{F}$ é a densidade de energia elástica para cristais líquidos nemáticos. A densidade de energia elástica é dada por

$$
\begin{aligned}
w_{F}= & \frac{1}{2}\left(K_{1}-K_{2}-K_{4}\right)\left(n_{i, i}\right)^{2}+\frac{1}{2} K_{2} n_{i, j} n_{i, j}+\frac{1}{2} K_{4} n_{i, j} n_{j, i} \\
& +\frac{1}{2}\left(K_{3}-K_{2}\right) n_{j} n_{i, j} n_{k} n_{i, k}
\end{aligned}
$$


onde $K_{i}, i=1, \ldots, 4$ são as constantes elásticas de Frank [57]. Também, pode ser mostrado que (ver Stewart [123])

$$
\left(\frac{\partial w_{F}}{\partial n_{i, j}}\right)_{, j}-\frac{\partial w_{F}}{\partial n_{i}}=\left(K_{1}-K_{2}\right) n_{j, j i}+K_{2} n_{i, j j}+\left(K_{3}-K_{2}\right)\left[\left(n_{j} n_{k} n_{i, k}\right)_{, j}-n_{j} n_{k, j} n_{k, i}\right] .
$$

Adotando uma aproximação constante para as constantes elásticas, ou seja,

$$
K=K_{1}=K_{2}=K_{3} \quad \text { e } \quad K_{4}=0
$$

a densidade de energia elástica (1.6) pode ser escrita como (mais detalhes sobre essa aproximação são fornecidos no livro de Stewart [123]):

$$
w_{F}=\frac{1}{2} K\|\nabla \mathbf{n}\|^{2}=\frac{1}{2} K n_{i, j} n_{i, j}
$$

e a equação (1.7) se reduz-se a

$$
\left(\frac{\partial w_{F}}{\partial n_{i, j}}\right)_{, j}-\frac{\partial w_{F}}{\partial n_{i}}=K n_{i, j j}
$$

em que $K>0$. Consequentemente, a equação de conservação de momento angular (1.5) pode ser escrita como:

$$
K n_{i, j j}+\widetilde{g}_{i}+G_{i}=\lambda n_{i}
$$

Nas equações acima, a vírgula denota a derivada parcial da variável precedente. Por exemplo, $n_{i, j}$ denota a derivada parcial da $i^{\text {th }}$-ésima componente do diretor com relação a $j^{\text {th }}$-ésima variável, ou seja, $n_{i, j}=\frac{\partial n_{i}}{\partial x_{j}}$. A derivada $\dot{v}_{i}$ representa a derivada material $\partial v_{i} / \partial t+v_{l} \partial v_{i} / \partial x_{l}$. Detalhes da interpretação física da derivada material podem ser encontrados em Aris [7](págs. 77-79). A função escalar $\lambda$ é um multiplicador de Lagrange que usualmente pode ser eliminado ou avaliado tomando o produto escalar da equação (1.5) com $\mathbf{n}$. As equações constitutivas para as tensões viscosas $\widetilde{t}_{i j}$ e o vetor $\widetilde{g}_{i}$ são dadas por

$$
\begin{aligned}
\widetilde{t}_{i j}= & \alpha_{1} n_{k} A_{k p} n_{p} n_{i} n_{j}+\alpha_{2} N_{i} n_{j}+\alpha_{3} n_{i} N_{j}+\alpha_{4} A_{i j} \\
& +\alpha_{5} n_{j} A_{i k} n_{k}+\alpha_{6} n_{i} A_{j k} n_{k}, \\
\widetilde{g}_{i}= & -\gamma_{1} N_{i}-\gamma_{2} A_{i p} n_{p},
\end{aligned}
$$

em que $\alpha_{i}(i=1,2, \ldots, 6)$, são as viscosidades de Leslie, o coeficiente $\gamma_{1}$ é a viscosidade rotacional e $\gamma_{2}$ é o coeficiente de torção. Em geral, as tensões viscosas $\widetilde{t}_{i j}$ não são simétricas.

O tensor taxa de deformação $A_{i j}$, o fluxo co-rotacional temporal do diretor $N_{i}$ (terminologia 
usada por Truesdell e Noll [135]) e o tensor vorticidade $W_{i j}$ são dados, respectivamente, por

$$
\begin{aligned}
A_{i j} & =\frac{1}{2}\left(v_{i, j}+v_{j, i}\right), \\
N_{i} & =\dot{n}_{i}-W_{i j} n_{j}, \quad W_{i j}=\frac{1}{2}\left(v_{i, j}-v_{j, i}\right) .
\end{aligned}
$$

O tensor das tensões para cristais líquidos nemáticos é dado por

$$
t_{i j}=-p \delta_{i j}-\frac{\partial w_{F}}{\partial n_{p, j}} n_{p, i}+\tilde{t}_{i j}
$$

\subsubsection{As viscosidades de Leslie}

As viscosidades nas tensões viscosas $\widetilde{t}_{i j}$ são obtidas quando deriva-se as equações dinâmicas e, em geral, não podem ser descritas fisicamente; uma exceção é a viscosidade $\alpha_{4}$ que é a usual viscosidade de fluidos newtonianos. No entanto, certas combinações lineares destas viscosidades podem ser identificadas experimentalmente e são conhecidas como viscosidades de Miesowicz $\eta_{i}$ introduzidas nas Seção 1.4.2. As viscosidades de Miesowicz pode ser escritas em termos das viscosidades de Leslie como [123]

$$
\begin{aligned}
\eta_{1} & =\frac{1}{2}\left(\alpha_{3}+\alpha_{4}+\alpha_{6}\right) \\
\eta_{2} & =\frac{1}{2}\left(-\alpha_{2}+\alpha_{4}+\alpha_{5}\right) \\
\eta_{3} & =\frac{1}{2} \alpha_{4} \\
\eta_{12} & =\alpha_{1}
\end{aligned}
$$

A viscosidade rotacional $\gamma_{1}$ e o coeficiente de torção $\gamma_{2}$ também podem ser re-escritos em termos das viscosidades de Leslie como

$$
\begin{aligned}
& \gamma_{1}=\alpha_{3}-\alpha_{2} \geq 0 \\
& \gamma_{2}=\alpha_{6}-\alpha_{5}
\end{aligned}
$$

Observe que as tensões viscosas $\widetilde{t}_{i j}$ dadas pela equação (1.12) envolve seis viscosidades de Leslie $\alpha_{i}, i=1,2, \ldots, 6$. No entanto, Parodi [103] propôs, via as relações de Onsager, a seguinte relação para os coeficientes de viscosidade

$$
\alpha_{6}-\alpha_{5}=\alpha_{2}+\alpha_{3}
$$

Com este resultado obtém-se

$$
\alpha_{6}=\alpha_{3}+\alpha_{2}+\alpha_{5}
$$


o que reduz o número de viscosidades independentes para cinco.

\subsection{Uma Revisão sobre Escoamentos de Cristais Líquidos}

Motivados pelas importantes aplicações industriais, muitas teorias têm sido desenvolvidas para melhorar a compreensão dos sistemas de cristal líquido. A principal teoria que tem sido frequentemente aplicada com sucesso nos problemas envolvendo cristais líquidos nemáticos é a teoria dinâmica de Ericksen-Leslie [123], embora outras teorias são amplamente utilizadas, por exemplo a Teoria de Doi [41].

As equações dinâmicas de Ericksen-Leslie introduzidas na Seção 1.5.2 vêm sendo utilizadas por muitos autores para a modelagem de escoamentos de cristais líquidos nemáticos. Por exemplo, Pikin [106] estudou um modelo unidimensional da influência do cisalhamento na orientação de um cristal líquido nemático e obteve algumas soluções aproximadas das equações de Ericksen-Leslie. Pikin provou a existência e estabilidade de diferentes tipos de comportamento do diretor dependendo dos parâmetros materiais. Baleo et al. [10], utilizando a hipótese de alta viscosidade e desprezando a elasticidade, obtiveram um modelo simplificado da teoria de Ericksen-Leslie, em que as equações resultantes são conhecidas como equações TIF " Transversely Isotropic Fluids" de Ericksen. Esse modelo simplificado foi então utilizado para resolver o escoamento de cristais líquidos poliméricos, vistos como fluidos anisotrópicos viscosos.

MacSithigh e Currie [85] também estudaram soluções aproximadas para a orientação do diretor para um forte cisalhamento, e em um outro trabalho Currie e MacSithigh [36] estudaram a estabilidade e dissipação dessas soluções. Por meio de estudos teóricos e experimentais, soluções semelhantes foram investigadas por Skarp e Carlsson [118] que consideraram a influência de um campo elétrico na orientação do diretor para forte cisalhamento.

Escoamentos com pressão controlada foi considerado por Atkin [8], que estudou a existência e unicidade de uma solução exata das equações de Ericksen-Leslie para o escoamento totalmente desenvolvido em um longo cilindro circular infinito. Posteriormente, Currie [35] discutiu o mesmo problema e encontrou soluções aproximadas para o diretor. Rey e Denn [110] obtiveram uma solução semelhante das equações de Ericksen-Leslie para escoamentos de fluxo prescrito entre fronteiras planares (Jeffery-Hamel flow).

Na literatura podem ser encontrados vários trabalhos, por exemplo [9, 10, 26, 69, 73, 105], sobre escoamentos de cristais líquidos nemáticos. Em muitos destes artigos, por exemplo ([9, 73]), a investigação do escoamento de cristal líquido nemático é realizada por meio de estudos de soluções analíticas aproximadas para as equações de Ericksen-Leslie. Em outros trabalhos, por exemplo [105], a investigação é obtida experimentalmente. Em geral, as equações governantes para escoamentos de cristais líquidos nemáticos são equações diferenciais acopladas que envolvem funções não-lineares, desse modo é difícil de se obter algum progresso analítico destas equações. Consequentemente, ótimas oportunidades surgem para o desenvolvimento de métodos numéricos capazes de resolver as equações não lineares que governam os escoamento de 
cristais líquidos nemáticos.

Alguns problemas em escoamentos de cristais líquidos nemáticos em geometrias confinadas tem sido investigados através da resolução das equações de Ericksen-Leslie numericamente. Por exemplo, Tu et al. [65] consideraram diferentes aproximações das constantes elásticas dos cristais líquidos para analisar o efeito da anisotropia elástica na orientação do diretor. Algumas geometrias complexas tem sido consideradas em estudos de escoamentos com pressão controlada; por exemplo, Chono e Tsuji [25] analisaram o escoamento em torno de um cilindro circular, enquanto que em outros estudos Chono et al. [26], estudaram o desenvolvimento espacial da orientação do diretor no escoamento de cristais líquidos nemáticos em regime “ tumbling". Outros estudos numéricos consideraram escoamentos transientes para descrever o comportamento de cristais líquidos nemáticos em regime "tumbling" [17, 67], ou para estudar o efeito da anisotropia elástica no escoamento e na orientação do diretor conforme apresentado por Tao e Feng [124].

Em trabalhos recentes, Carou et. al. [19, 20, 21] encontraram uma solução analítica para o problema do escoamento de um fino filme de cristal líquido nemático em um canal com lenta variação de largura. Nesses trabalhos, foram realizadas algumas aproximações, por exemplo a aproximação de "thin film fluids" [2, 40, 78, 97, 98, 117], para identificar situações em que exista uma dissociação entre o escoamento e o diretor, permitindo encontrar soluções explícitas para o campo de velocidades e para o diretor.

Buscando o desenvolvimento de novas aplicações dos cristais líquidos, os cristais líquidos poliméricos (LCPs) tem atraído a atenção de muitos pesquisadores devido a vasta possibilidade de aplicações desses materiais [111]. Os cristais líquidos poliméricos são utilizados no processamento de peças eletrônicas devido à suas propriedades mecânicas tais como resistência e rigidez, maleabilidade e alta resistência térmica e também são utilizados com sucesso na área de fibras de alta resistência. Recentemente, Yamamoto et al. [143], utilizaram um modelo modificado de Doi para investigar o comportamento da orientação molecular de cristais líquidos poliméricos em escoamentos complexos. Fu et al. [61] utilizando a equação de Doi-Hess analisaram numericamente o efeito do campo magnético na orientação de cristais líquidos poliméricos sob escoamento cisalhante simples.

Devido a sua importância no desenvolvimento de novas aplicações dos cristais líquidos, tais como polímeros com cristais líquidos dispersos (Polymer-Dispersed Liquid Crystals - PDLCs)(ver Figura $1.9^{2}$ ), as propriedades dos cristais líquidos estão sendo amplamente investigadas nas últimas décadas. PDLCs são simplesmente uma aplicação combinada de polímeros e cristais líquidos nemáticos. Esses sistemas consistem de gotículas de cristais líquidos nemáticos que estão dispersas em uma fina matriz polimérica. O material resultante é uma espécie de polímero com gotas de cristal líquido preenchendo os buracos (" swiss cheese" polymer). A configuração

\footnotetext{
${ }^{2}$ Figura extraída no dia 05/07/2011 do filme: High transmission LCD shutter by GPEG displays no site: http://wn.com/Polymer-Dispersed-Liquid-Crystals.
} 
do diretor no interior de cada gota pode ser influenciada por um campo externo orientado e, dessa forma é possível variar a intensidade da luz transmitida, ou seja, o filme pode se tornar transparente ou opaco [3]. Estudos teóricos sobre gotículas de cristal líquido nemático podem ser encontrados nos trabalhos de Romano et al. [113], Virga [137] e Rey [109]. Trabalhos experimentais de gotículas de cristais líquidos nemáticos podem ser encontrados nos trabalhos de Vandenbrouck et al. [136] e Lin et al. [84].
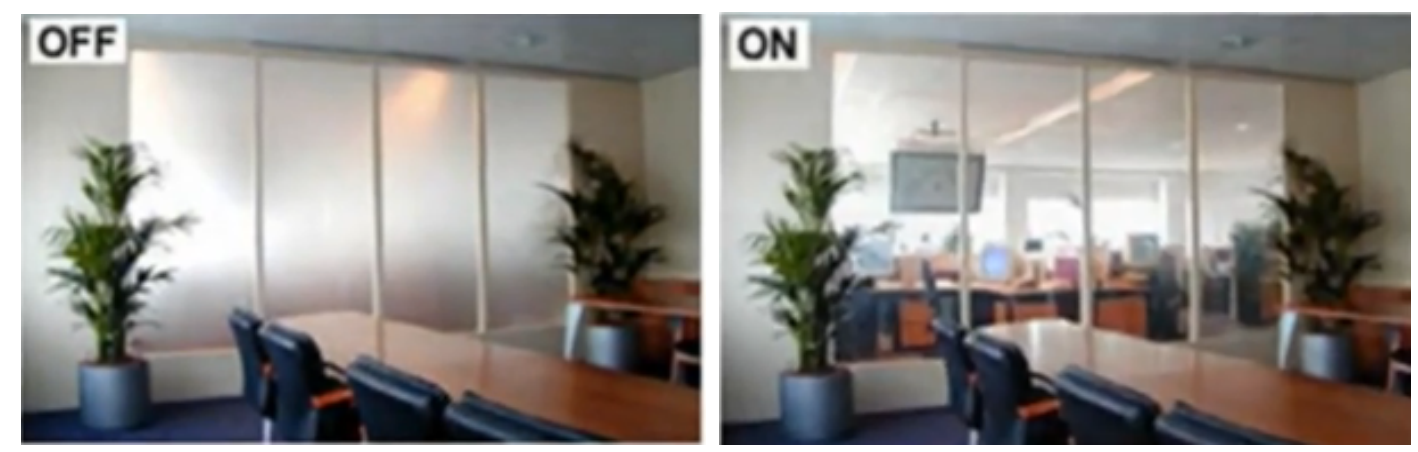

Figura 1.9: As fotos mostram um persiana de LCD. No estado "OFF" sem nenhuma tensão aplicada o cristal líquido espalha a luz incidente e a persiana fica opaca, aplicando uma tensão (estado "ON"), as moléculas de cristal líquido se alinham e a persiana torna-se transparente.

\subsection{Uma Revisão sobre o Método GENSMAC}

Nesse trabalho, a principal técnica abordada para a simulação de escoamentos de cristais líquidos nemáticos é baseada na metodologia GENSMAC [134] para o caso bidimensional e para o caso tridimensional é utilizada a metodologia GENSMAC3D [127]. O método GENSMAC desenvolvido por Tomé e McKee [134] é uma variação de técnicas numéricas desenvolvidas para a simulação de escoamentos de fluidos com superfícies livres. Umas das primeiras técnicas desenvolvidas foi o método MAC (Marker-And-Cell)[68, 141] que foi introduzido por Harlow e Welch, em Los Alamos no começo da década de 60. O método MAC é uma técnica baseada em diferenças finitas que emprega variáveis primitivas (velocidade e pressão) para a simulação de escoamentos de fluidos incompressíveis. A partir do método MAC outros métodos numéricos foram desenvolvidos. Em 1970, Amsden e Harlow [4] desenvolveram um método MAC simplificado, denominado SMAC (Simplified-Marker-And-Cell) cuja característica principal é a divisão do ciclo computacional em duas partes: velocidade e pressão, de modo que não existe um processo iterativo envolvendo velocidade e pressão, fazendo assim com que algumas dificuldades do método MAC original [68] fossem eliminadas.

Outras variações do método MAC, foram apresentadas na década de 70 como o método ALE [70]; na década de 80 no trabalho de Miyata [92]; e na década de 90 no método GENSMAC [134]. Recentemente, McKee et al. [88] descreveram a história, interpretação, variações e aplicações do método MAC. 
Tomé e McKee [134] implementaram o método GENSMAC com o objetivo de resolver problemas de escoamentos transientes incompressíveis com superfícies livres, mas diversas variações do método surgiram. Essas variações são apresentadas em: Ferreira et al. [53] que realizaram simulações de problemas turbulentos com superfícies livres, Ferreira et al. [54] que adaptaram o método MAC para calcular escoamentos confinados e com superfícies livres a altos e baixos números de Reynolds, Tomé et al. [132] que aplicaram as técnicas do método MAC na simulação de escoamentos axissimétrico com superfícies livres utilizando fluido do tipo Oldroyd-B, Mangiavacchi et al. [86] que implementaram novas técnicas em escoamentos axissimétrico e bidimensionais com superfícies livres quando a tensão superficial é importante. Recentemente, Ferreira e seus colaboradores [50, 51, 52] aplicaram estratégias upwind na metodologia GENSMAC, Tomé e seus colaboradores [129, 128] apresentaram resultados numéricos para problemas viscoelásticos com superfícies livres. Cruz et al. [32] adaptaram o método GENSMAC3D para calcular escoamentos tridimensionais confinados de cristais líquidos nemáticos sob efeito de forte campo magnético, entre outras novas aplicações do método. Recentemente, Cruz et al. [33] fizeram uma adaptação no método GENSMAC e desenvolveram um método de diferenças finitas para resolver escoamentos de cristais líquidos nemáticos em duas dimensões.

\subsection{Linhas Gerais da Tese}

O objetivo principal desta tese é o desenvolvimento de métodos numéricos para simular escoamentos de cristais líquidos nemáticos governados pelas equações dinâmicas de Ericksen-Leslie [45, 81, 123]. Fundamentado na teoria dinâmica de Ericksen-Leslie e na metodologia GENSMAC este texto é dividido em três partes principais, que apresentam resultados originais a esta tese.

No Capítulo 2 deste trabalho, exibe-se o desenvolvimento de um método numérico para simular escoamentos tridimensionais de cristais líquidos nemáticos sob o efeito de um forte campo magnético. Sob a hipótese de que o campo magnético é muito forte, as equações de conservação de momento angular (1.5) para o diretor são desprezadas. Apresenta-se as soluções analíticas para o escoamento tridimensional totalmente desenvolvido entre duas placas paralelas quando um forte campo magnético $\mathbf{H}=H\left(0, \operatorname{sen} \phi_{0}, \cos \phi_{0}\right)$ ( $\phi_{0}$ um ângulo constante) é aplicado através das placas. A seguir, as equações dinâmicas para um forte campo magnético, respectivas equações na forma adimensional e as condições de contorno são apresentadas. Descreve-se passo-a-passo o método numérico desenvolvido, que é baseado na metodologia GENSMAC3D [127]. Apresenta-se os resultados da validação da técnica numéria desenvolvida, bem como os resultados obtidos da simulação do escoamento em um canal tridimensional.

A seguir, no Capítulo 3 apresenta-se todo o desenvolvimento de um método numérico para resolver as equações dinâmicas de Ericksen-Leslie em duas dimensões. Descreve-se as equações básicas que governam escoamentos bidimensionais de cristais líquidos nemáticos, e em seguida aborda-se a formulação matemática para a obtenção do método numérico bidimensional. Uti- 
lizando a técnica de diferenças finitas em uma malha deslocada bidimensional, apresenta-se as aproximações das equações que descrevem o método numérico. Na Seção 3.5, empregando a hipótese de escoamento totalmente desenvolvido obtém-se equações simplificadas para o escoamento em um canal bidimensional. Essas equações simplificadas são equações diferenciais acopladas que envolvem funções não lineares de $\phi$, e assim nenhum progresso analítico pode ser feito. Para contornar essa dificuldade, é feita uma aproximação que depende do tamanho do ângulo do diretor $\phi$, e desse modo o sistema de equações resultantes foi resolvido analiticamente e obteve-se expressões para a velocidade $u$, para o ângulo de orientação do diretor $\phi$ e consequentemente, obteve-se expressões para as componentes do tensor das tensões não-newtoniano $\Phi_{i j}$. A partir da soluções analíticas obtidas, apresenta-se os resultados da validação da técnica numérica desenvolvida.

Com o intuito de demonstrar a capacidade do método numérico desenvolvido no Capítulo 3 em simular escoamentos bidimensionais de cristais líquidos nemáticos, a técnica numérica foi aplicada para simular escoamentos de cristais líquidos nemáticos em várias geometrias complexas. Por exemplo, foi realizada a simulação numérica do escoamento através de uma contração planar 4:1. Esse problema é um dos instrumentos clássicos utilizados no desenvolvimento de métodos numéricos para a simulação de fluidos não-newtonianos. Resultados numéricos foram obtidos, e uma variedade de padrões do escoamento e da orientação dos diretores é apresentada no Capítulo 4. Os escoamentos de um cristal líquido nemático em um L-canal (canal em forma de L) e em uma expansão planar 1:4 também são considerados no Capítulo 4.

O último Capítulo desta tese é dedicado às considerações finais e trabalhos futuros, enquanto que no Apêndice A, os parâmetros físicos dos cristais líquidos nemáticos são apresentados em Tabelas.

Alguns dos resultados desta tese já foram publicados na literatura. Por exemplo, os resultados obtidos no Capítulo 2 foram reunidos no artigo

- Cruz et al., A numerical method for solving the dynamic three-dimensional Ericksen-Leslie equations for nematic liquid crystals subject to a strong magnetic field. Journal of Non-Newtonian Fluid Mechanics, 165: 113-157, 2010.

Os resultados obtidos nos Capítulos 3 e 4 foram reunidos em dois artigos

- Cruz et al., Numerical investigation of director orientation and flow of nematic liquid crystal in a planar 1:4 expansion, Journal of Mechanics of Materials and Structures, em impressão, 2011

- Cruz et al., Numerical solution of the Ericksen-Leslie dynamic equations for two-dimensional nematic liquid crystal flows, artigo submetido ao Journal of Computational Physics, 2011.

Alguns resultados dos trabalhos desenvolvidos nessa tese foram apresentados em vários congressos nacionais e internacionais, como segue: 
1. Cruz et. al., Numerical Simulation of Two-Dimensional Nematic Liquid Crystal Flows, Congreso de Métodos Numéricos en Ingeniería - SEMNI - 29 junio al 2 de julio 2009, Barcelona - España

2. Cruz et. al., Simulação Numérica de Escoamentos Tri-dimensionais de Cristais Líquidos Nemáticos Sujeitos a um Forte Campo Magnético, Congresso Nacional de Matemática Aplicada e Computacional - CNMAC - Setembro 2009, Cuiabá - MT, Brasil

3. Cruz et. al., Numerical Investigation of Two-dimensional Flows of Nematic Liquid Crystals, 20th International Congress of Mechanical Engineering - COBEM - November 15-20, 2009, Gramado - RS, Brasil

4. Tomé et. al., Numerical Investigation of Director Orientation and Flow of Nematic Liquid Crystal in a Planar 1:4 Expansion, 11th Pan-American Congress of Applied Mechanics PACAM XI - January 04-08, 2010, Foz do Iguaçu - PR, Brasil

5. Cruz et. al., Numerical Simulation of Director Orientation of Nematic Liquid Crystal in Tumbling Flows, V European Conference on Computational Fluid Dynamics - ECCOMAS CFD - June 14-17, 2010, Lisbon, Portugal

6. Cruz et. al., Numerical Simulation of Nematic Liquid Crystal Flows with different Tumbling Parameters, 21st International Congress of Mechanical Engineering - COBEM October 24-28, 2011, Natal, RN, Brasil 
CAPÍTULO

\section{Escoamentos de Cristais Líquidos Nemáticos sob Forte Campo Magnético}

Nesse Capítulo é apresentado um método numérico para simular escoamentos tridimensionais de cristais líquidos nemáticos, sob o efeito de um forte campo magnético, governados pelas equações dinâmicas de Ericksen-Leslie. Se o campo magnético for suficientemente forte então o diretor permanece fixo no escoamento e as equações de conservação de momento angular (1.5) podem ser desprezadas (como foi sugerido por Leslie [83]). Nesse caso, as equações básicas que governam escoamentos incompressíveis tridimensionais, isotérmicos descritos pelas equações dinâmicas de Ericksen-Leslie são dadas pelas equações (1.2) e (1.4) que são resolvidas por um método numérico baseado na metodologia GENSMAC3D [127]. A técnica numérica desenvolvida nesse Capítulo foi implementada no código Freeflow3D e validada utilizando uma solução analítica do escoamento desenvolvido entre duas placas paralelas. Utilizando refinamento de malha, resultados de convergência do método numérico são apresentados bem como resultados numéricos obtidos na simulação do escoamento de cristal liquido nemático em um canal tridimensional. 


\subsection{Escoamento entre Duas Placas Paralelas}

Considere o escoamento tridimensional de um cristal líquido nemático entre duas placas paralelas. Um forte campo magnético $\mathbf{H}$ é aplicado entre as placas de modo que o diretor permanece fixo em uma direção paralela a direção do campo magnético (ver Figura 2.1).

$\mathrm{O}$ diretor $\mathbf{n}$ e a velocidade $\mathbf{v}$ podem ser escritos nas formas gerais

$$
\begin{aligned}
& \mathbf{n}=(\operatorname{sen} \theta, \operatorname{sen} \phi \cos \theta, \cos \phi \cos \theta), \theta=\theta(x, y, z, t), \phi=\phi(x, y, z, t) \\
& \mathbf{v}=(u(x, y, z, t), v(x, y, z, t), w(x, y, z, t))
\end{aligned}
$$

onde $\phi$ e $\theta$ são ângulos de orientação do diretor (ver Figura 1.8).

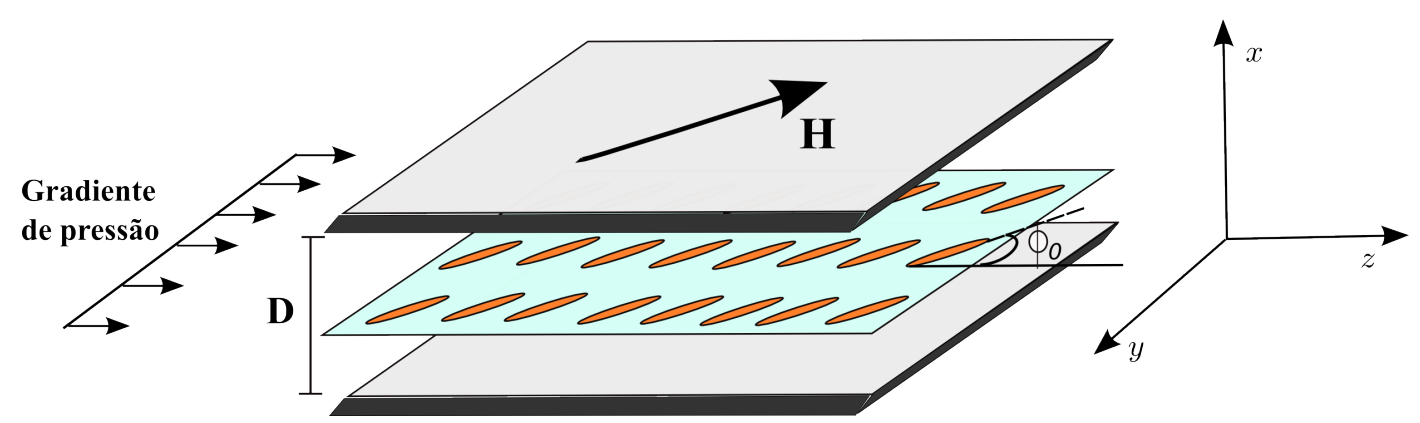

Figura 2.1: Descrição do escoamento 3D entre duas placas paralelas.

Considere o escoamento desenvolvido entre duas placas paralelas conforme mostrado na Figura 2.1 em que um forte campo magnético $\mathbf{H}=H\left(0, \operatorname{sen} \phi_{0}, \cos \phi_{0}\right), \phi_{0}$ fixo, é aplicado entre as placas. Nessas condições, assume-se que as seguintes hipóteses são válidas:

$$
\begin{aligned}
& \theta=0, \quad \phi=\phi_{0}, \quad \frac{\partial}{\partial t} \equiv \frac{\partial}{\partial y} \equiv \frac{\partial}{\partial z} \equiv 0, \quad u=0 \\
& v=v(x), \quad w=w(x) \quad \text { e } \quad p_{, z}=\mathcal{A}(\text { constante })
\end{aligned}
$$

Assume-se ainda que as velocidades $v(x)$ e $w(x)$ obedecem a condição não-deslizamento nas paredes das placas $(w(0)=w(D)=v(0)=v(D)=0)$ e que um gradiente de pressão $\left(p_{, z}\right)$ é aplicado na direção $z$.

Esse problema foi estudado por Stewart [123] que obteve as soluções analíticas expressas em termos das viscosidades de Miesowicz dadas por:

$$
\begin{aligned}
v(x) & =-\frac{\left(\eta_{3}-\eta_{1}\right)}{2 \eta_{3} \eta_{1}} \operatorname{sen} \phi_{0} \cos \phi_{0} \mathcal{A} x(D-x), \\
w(x) & =-\frac{\left(\eta_{1} \operatorname{sen}^{2} \phi_{0}+\eta_{3} \cos ^{2} \phi_{0}\right)}{2 \eta_{3} \eta_{1}} \mathcal{A} x(D-x),
\end{aligned}
$$


em que, utilizando a relação de Parodi (1.24) na equação (1.17), tem-se

$$
\begin{aligned}
& \eta_{1}=\frac{1}{2}\left(\alpha_{2}+2 \alpha_{3}+\alpha_{4}+\alpha_{5}\right) \\
& \eta_{3}=\frac{1}{2} \alpha_{4}
\end{aligned}
$$

e a pressão é dada por

$$
p=p(z)=\mathcal{A} z+p_{0}, \quad p_{0} \text { uma constante. }
$$

Detalhes de como obter essas soluções analíticas para escoamentos de cristais líquidos nemáticos em um canal tridimensional sob forte campo magnético são fornecidos em [123]. Estas soluções teóricas foram confirmadas experimentalmente por Pieranski e Guyon [105]. A viscosidade $\eta_{3}=\frac{1}{2} \alpha_{4}$ corresponde a viscosidade isotrópica newtoniana. De fato, as soluções (2.4) e (2.5) reduzem ao caso de fluido newtoniano [123] quando tomamos $\eta_{1}=\eta_{3}$ e assim,

$$
v \equiv 0, \quad w=-\frac{\mathcal{A}}{\alpha_{4}} x(D-x)
$$

\subsection{Equações Dinâmicas para um Forte Campo Magné- tico}

Nesta seção, as equações dinâmicas para escoamentos de cristais líquidos nemáticos sujeito a um forte campo magnético são apresentadas.

Assume-se que $\theta \equiv 0$ e que $\phi=\phi_{0}$ é um ângulo constante determinado pela direção do campo magnético aplicado conforme mostrado na Figura 2.1. Logo, o diretor toma a forma

$$
\mathbf{n}=\left(0, \operatorname{sen} \phi_{0}, \cos \phi_{0}\right)
$$

e o gradiente do diretor pode ser ignorado (desde que $\phi_{0}$ é constante). Logo, a equação de conservação de momento angular (1.5) para o diretor também pode ser desprezada e os efeitos da força gravitacional são desprezados.

\subsubsection{Equações de Conservação de Massa e de Quantidade de Movi- mento}

Utilizando coordenadas cartesianas, a equação de conservação de massa (1.3) é escrita como

$$
u_{, x}+v_{, y}+w_{, z}=0 .
$$


e as componentes da equação de conservação de quantidade de movimento (1.4) são dadas por (usando a condição (2.11) onde necessário),

$$
\begin{aligned}
\rho\left(u_{, t}+u u_{, x}+v u_{, y}+w u_{, z}\right) & =-p_{, x}+\widetilde{t}_{x x, x}+\widetilde{t}_{x y, y}+\widetilde{t}_{x z, z}, \\
\rho\left(v_{, t}+u v_{, x}+v v_{, y}+w v_{, z}\right) & =-p_{, y}+\widetilde{t}_{y x, x}+\widetilde{t}_{y y, y}+\widetilde{t}_{y z, z} \\
\rho\left(w_{, t}+u w_{, x}+v w_{, y}+w w_{, z}\right) & =-p_{, z}+\widetilde{t}_{z x, x}+\widetilde{t}_{z y, y}+\widetilde{t}_{z z, z} .
\end{aligned}
$$

Introduzindo o diretor (2.10) nas equações (1.12), (1.14) e (1.15), pode ser mostrado que as componentes das tensões viscosas são dadas por:

$$
\begin{aligned}
& \widetilde{t}_{x x}=\alpha_{4} u_{, x} \\
& \tilde{t}_{x y}=\frac{1}{2} \alpha_{2}\left[\operatorname{sen}^{2} \phi_{0}\left(v_{, x}-u_{, y}\right)+\operatorname{sen} \phi_{0} \cos \phi_{0}\left(w_{, x}-u_{, z}\right)\right] \\
& +\frac{1}{2} \alpha_{4}\left(u_{, y}+v_{, x}\right)+\frac{1}{2} \alpha_{5}\left[\operatorname{sen}^{2} \phi_{0}\left(u_{, y}+v_{, x}\right)\right. \\
& \left.+\operatorname{sen} \phi_{0} \cos \phi_{0}\left(u_{, z}+w_{, x}\right)\right] \text {, } \\
& \tilde{t}_{x z}=\frac{1}{2} \alpha_{2}\left[\cos ^{2} \phi_{0}\left(w_{, x}-u_{, z}\right)+\operatorname{sen} \phi_{0} \cos \phi_{0}\left(v_{, x}-u_{, y}\right)\right] \\
& +\frac{1}{2} \alpha_{4}\left(u_{, z}+w_{, x}\right)+\frac{1}{2} \alpha_{5}\left[\cos ^{2} \phi_{0}\left(u_{, z}+w_{, x}\right)\right. \\
& \left.+\operatorname{sen} \phi_{0} \cos \phi_{0}\left(u_{, y}+v_{, x}\right)\right] \text {, } \\
& \tilde{t}_{y x}=\frac{1}{2} \alpha_{3}\left[\operatorname{sen}^{2} \phi_{0}\left(v_{, x}-u_{, y}\right)+\operatorname{sen} \phi_{0} \cos \phi_{0}\left(w_{, x}-u_{, z}\right)\right] \\
& +\frac{1}{2} \alpha_{4}\left(u_{, y}+v_{, x}\right)+\frac{1}{2} \alpha_{6}\left[\operatorname{sen}^{2} \phi_{0}\left(v_{, x}+u_{, y}\right)\right. \\
& \left.+\operatorname{sen} \phi_{0} \cos \phi_{0}\left(u_{, z}+w_{, x}\right)\right] \text {, } \\
& \tilde{t}_{y y}=\alpha_{1} \operatorname{sen}^{2} \phi_{0}\left[w_{, z} \cos ^{2} \phi_{0}+v_{, y} \operatorname{sen}^{2} \phi_{0}+\frac{1}{2}\left(v_{, z}+w_{, y}\right) \operatorname{sen}\left(2 \phi_{0}\right)\right] \\
& -\left(\alpha_{2}+\alpha_{3}\right) \operatorname{sen} \phi_{0} \cos \phi_{0}\left[\frac{1}{2}\left(v_{, z}-w_{, y}\right)\right]+\alpha_{4} v_{, y} \\
& +\left(\alpha_{5}+\alpha_{6}\right)\left[v_{, y} \operatorname{sen}^{2} \phi_{0}+\frac{1}{2} \operatorname{sen} \phi_{0} \cos \phi_{0}\left(w_{, y}+v_{, z}\right)\right],
\end{aligned}
$$




$$
\begin{aligned}
& \tilde{t}_{y z}=\alpha_{1} \operatorname{sen} \phi_{0} \cos \phi_{0}\left[w_{, z} \cos ^{2} \phi_{0}+v_{, y} \operatorname{sen}^{2} \phi_{0}+\frac{1}{2}\left(w_{, y}+v_{, z}\right) \operatorname{sen}\left(2 \phi_{0}\right)\right] \\
& -\left(\alpha_{2} \cos ^{2} \phi_{0}-\alpha_{3} \operatorname{sen}^{2} \phi_{0}\right)\left[\frac{1}{2}\left(v_{, z}-w_{, y}\right)\right]+\frac{1}{2} \alpha_{4}\left(w_{, y}+v_{, z}\right) \\
& +\frac{1}{2}\left(\alpha_{5} \cos ^{2} \phi_{0}+\alpha_{6} \operatorname{sen}^{2} \phi_{0}\right)\left(w_{, y}+v_{, z}\right)+\operatorname{sen} \phi_{0} \cos \phi_{0}\left(\alpha_{5} v_{, y}+\alpha_{6} w_{, z}\right) \text {, } \\
& \tilde{t}_{z x}=\frac{1}{2} \alpha_{3}\left[\cos ^{2} \phi_{0}\left(w_{, x}-u_{, z}\right)+\operatorname{sen} \phi_{0} \cos \phi_{0}\left(v_{, x}-u_{, y}\right)\right] \\
& +\frac{1}{2} \alpha_{4}\left(u_{, z}+w_{, x}\right)+\frac{1}{2} \alpha_{6}\left[\operatorname { c o s } ^ { 2 } \phi _ { 0 } \left(u_{, z}\right.\right. \\
& \left.\left.+w_{, x}\right)+\operatorname{sen} \phi_{0} \cos \phi_{0}\left(v_{, x}+u_{, y}\right)\right] \text {, } \\
& \tilde{t}_{z y}=\alpha_{1} \operatorname{sen} \phi_{0} \cos \phi_{0}\left[w_{, z} \cos ^{2} \phi_{0}+v_{, y} \operatorname{sen}^{2} \phi_{0}+\frac{1}{2}\left(w_{, y}+v_{, z}\right) \operatorname{sen}\left(2 \phi_{0}\right)\right] \\
& -\left(\alpha_{3} \cos ^{2} \phi_{0}-\alpha_{2} \operatorname{sen}^{2} \phi_{0}\right)\left[\frac{1}{2}\left(v_{, z}-w_{, y}\right)\right]+\frac{1}{2} \alpha_{4}\left(w_{, y}+v_{, z}\right) \\
& +\frac{1}{2}\left(\alpha_{5} \operatorname{sen}^{2} \phi_{0}+\alpha_{6} \cos ^{2} \phi_{0}\right)\left(w_{, y}+v_{, z}\right)+\operatorname{sen} \phi_{0} \cos \phi_{0}\left(\alpha_{5} w_{, z}+\alpha_{6} v_{, y}\right) \text {, } \\
& \tilde{t}_{z z}=\alpha_{1} \cos ^{2} \phi_{0}\left[w_{, z} \cos ^{2} \phi_{0}+v_{, y} \operatorname{sen}^{2} \phi_{0}+\frac{1}{2}\left(w_{, y}+v_{, z}\right) \operatorname{sen}\left(2 \phi_{0}\right)\right] \\
& -\left(\alpha_{2}+\alpha_{3}\right) \operatorname{sen} \phi_{0} \cos \phi_{0}\left[\frac{1}{2}\left(w_{, y}-v_{, z}\right)\right]+\alpha_{4} w_{, z} \\
& +\left(\alpha_{5}+\alpha_{6}\right)\left[w_{, z} \cos ^{2} \phi_{0}+\frac{1}{2} \operatorname{sen} \phi_{0} \cos \phi_{0}\left(w_{, y}+v_{, z}\right)\right] .
\end{aligned}
$$

Das equações acima, observa-se que $\tilde{t}_{i j} \neq \tilde{t}_{j i}$, e portanto o tensor das tensões viscosas não é simétrico. A substituição das expressões acima nas equações (2.12) - (2.14) obtém-se as equações dinâmicas originadas das equações de quantidade de movimento (1.4). Logo, as equações dinâmicas de Ericksen-Leslie reduzem-se para quatro equações, (2.11), (2.12), (2.13) e (2.14) para determinar quatro incógnitas $u, v, w$ e $p$.

A primeira diferença de tensões normais $\sigma_{1}=\tilde{t}_{x x}-\tilde{t}_{y y}$ é não nula: esta é uma característica do comportamento não-newtoniano dos cristais líquidos nemáticos [123]. 


\subsubsection{Caso especial para $\phi_{0} \equiv 0$}

Para o caso particular em que $\phi_{0} \equiv 0$, as tensões viscosas $\tilde{t}_{i j}$ reduzem-se à

$$
\begin{aligned}
\tilde{t}_{x x} & =\alpha_{4} u_{, x}, \\
\widetilde{t}_{x y} & =\frac{1}{2} \alpha_{4}\left(u_{, y}+v_{, x}\right), \\
\widetilde{t}_{x z} & =\frac{1}{2}\left(\alpha_{2}+\alpha_{4}+\alpha_{5}\right) w_{, x}+\frac{1}{2}\left(-\alpha_{2}+\alpha_{4}+\alpha_{5}\right) u_{, z}, \\
\widetilde{t}_{y x} & =\frac{1}{2} \alpha_{4}\left(u_{, y}+v_{, x}\right), \\
\widetilde{t}_{y y} & =\alpha_{4} v_{, y} \\
\widetilde{t}_{y z} & =\frac{1}{2}\left(-\alpha_{2}+\alpha_{4}+\alpha_{5}\right) v_{, z}+\frac{1}{2}\left(\alpha_{2}+\alpha_{4}+\alpha_{5}\right) w_{, y} \\
\widetilde{t}_{z x} & =\frac{1}{2}\left(\alpha_{3}+\alpha_{4}+\alpha_{6}\right) w_{, x}+\frac{1}{2}\left(-\alpha_{3}+\alpha_{4}+\alpha_{6}\right) u_{, z}, \\
\widetilde{t}_{z y} & =\frac{1}{2}\left(-\alpha_{3}+\alpha_{4}+\alpha_{6}\right) v_{, z}+\frac{1}{2}\left(\alpha_{3}+\alpha_{4}+\alpha_{6}\right) w_{, y}, \\
\widetilde{t}_{z z} & =\left(\alpha_{1}+\alpha_{4}+\alpha_{5}+\alpha_{6}\right) w_{, z} .
\end{aligned}
$$

\subsection{Forma Adimensional das Equações Dinâmicas}

Nesse trabalho considera-se escoamentos cartesianos tridimensionais com $\mathbf{x}=(x, y, z, t), \mathbf{v}=$ $(u, v, w)$. Com o objetivo de simplificar a notação, $u(x, y, z, t)$ é denotada por $u$ e utiliza-se uma notação semelhante para $v(x, y, z, t), w(x, y, z, t)$ e $p(x, y, z, t)$.

Para a adimensionalização das equações, as unidades escalares de comprimento e de velocidade são denotadas por $L$ e $U$, respectivamente. Seguindo a metodologia apresentada em [133], a adimensionalização pode ser feita a partir da seguinte mudança de variáveis:

$$
\mathbf{x}=L \widetilde{\mathbf{x}}, \quad t=L U^{-1} \widetilde{t}, \quad \mathbf{v}=U \widetilde{\mathbf{v}}, \quad p=\rho U^{2} \widetilde{p} \quad \text { e } \tilde{t}_{i j}=\left(U^{-1} L / \eta\right) \mathbf{S}
$$

Neste trabalho, $\eta$ corresponde a viscosidade isotrópica newtoniana e é igual a $\eta_{3}=\frac{1}{2} \alpha_{4}$.

Introduzindo essas variáveis nas equações (2.12)-(2.14) obtém-se as seguintes equações de conservação de quantidade de movimento na forma adimensional:

$$
\begin{aligned}
u_{t}+u u_{, x}+v u_{, y}+w u_{, z} & =-p_{, x}+\frac{1}{R e}\left(S_{x x, x}+S_{x y, y}+S_{x z, z}\right), \\
v_{t}+u v_{, x}+v v_{, y}+w v_{, z} & =-p_{, y}+\frac{1}{R e}\left(S_{y x, x}+S_{y y, y}+S_{y z, z}\right), \\
w_{t}+u w_{, x}+v w_{, y}+w w_{, z} & =-p_{, z}+\frac{1}{R e}\left(S_{z x, x}+S_{z y, y}+S_{z z, z}\right),
\end{aligned}
$$

em que os acentos $\sim$ foram omitidos e $R e=L U \frac{\rho}{\eta}$ denota o número de Reynolds. 
A equação de conservação de massa (2.11) na forma adimensional é dada por

$$
u_{, x}+v_{, y}+w_{, z}=0
$$

Estas quatro equações formam o conjunto completo das equações dinâmicas e devem ser resolvidas sujeitas à condições de contornos apropriadas para encontrar as soluções $u, v, w$, e $p$.

\subsection{Método Numérico}

Para resolver as equações (2.34)-(2.37) primeiramente deve-se escrever as componentes das tensões $S_{i j}$ da seguinte forma:

$$
\begin{aligned}
& S_{x x}=\left[2 u_{, x}+\Phi_{x x}\right], \\
& S_{x y}=\left[\left(u_{, y}+v_{, x}\right)+\Phi_{x y}\right], \\
& S_{x z}=\left[\left(u_{, z}+w_{, x}\right)+\Phi_{x z}\right], \\
& S_{y x}=\left[\left(u_{, y}+v_{, x}\right)+\Phi_{y x}\right], \\
& S_{y y}=\left[2 v_{, y}+\Phi_{y y}\right], \\
& S_{y z}=\left[\left(v_{, z}+w_{, y}\right)+\Phi_{y z}\right], \\
& S_{z x}=\left[\left(u_{, z}+w_{, x}\right)+\Phi_{z x}\right], \\
& S_{z y}=\left[\left(v_{, z}+w_{, y}\right)+\Phi_{z y}\right], \\
& S_{z z}=\left[2 w_{, z}+\Phi_{z z}\right] .
\end{aligned}
$$

onde as funções $\Phi_{x x}, \cdots, \Phi_{z z}$, são as componentes do tensor das tensões não-newtoniano $\boldsymbol{\Phi}_{\mathrm{ij}}$ e são dadas a seguir por

$$
\Phi_{x x}=0,
$$




$$
\begin{aligned}
& \Phi_{x y}=\frac{1}{2} \alpha_{2}\left[\operatorname{sen}^{2} \phi_{0}\left(v_{, x}-u_{, y}\right)+\operatorname{sen} \phi_{0} \cos \phi_{0}\left(w_{, x}-u_{, z}\right)\right] \\
& +\frac{1}{2} \alpha_{5}\left[\operatorname{sen}^{2} \phi_{0}\left(v_{, x}+u_{, y}\right)+\operatorname{sen} \phi_{0} \cos \phi_{0}\left(u_{, z}+w_{, x}\right)\right] \text {, } \\
& \Phi_{x z}=\frac{1}{2} \alpha_{2}\left[\cos ^{2} \phi_{0}\left(w_{, x}-u_{, z}\right)+\operatorname{sen} \phi_{0} \cos \phi_{0}\left(v_{, x}-u_{, y}\right)\right] \\
& +\frac{1}{2} \alpha_{5}\left[\cos ^{2} \phi_{0}\left(u_{, z}+w_{, x}\right)+\operatorname{sen} \phi_{0} \cos \phi_{0}\left(v_{, x}+u_{, y}\right)\right] \text {, } \\
& \Phi_{y x}=\frac{1}{2} \alpha_{3}\left[\operatorname{sen}^{2} \phi_{0}\left(v_{, x}-u_{, y}\right)+\operatorname{sen} \phi_{0} \cos \phi_{0}\left(w_{, x}-u_{, z}\right)\right] \\
& +\frac{1}{2} \alpha_{6}\left[\operatorname{sen}^{2} \phi_{0}\left(v_{, x}+u_{, y}\right)+\operatorname{sen} \phi_{0} \cos \phi_{0}\left(u_{, z}+w_{, x}\right)\right], \\
& \Phi_{y y}=\alpha_{1} \operatorname{sen}^{2} \phi_{0}\left[w_{, z} \cos ^{2} \phi_{0}+v_{, y} \operatorname{sen}^{2} \phi_{0}+\frac{1}{2}\left(w_{, y}+v_{, z}\right) \operatorname{sen}\left(2 \phi_{0}\right)\right] \\
& +\left(\alpha_{2}+\alpha_{3}\right) \operatorname{sen} \phi_{0} \cos \phi_{0}\left[\frac{1}{2}\left(w_{, y}-v_{, z}\right)\right] \\
& +\left(\alpha_{5}+\alpha_{6}\right)\left[v_{, y} \operatorname{sen}^{2} \phi_{0}+\frac{1}{2} \operatorname{sen} \phi_{0} \cos \phi_{0}\left(w_{, y}+v_{, z}\right)\right], \\
& \Phi_{y z}=\alpha_{1} \operatorname{sen} \phi_{0} \cos \phi_{0}\left[w_{, z} \cos ^{2} \phi_{0}+v_{, y} \operatorname{sen}^{2} \phi_{0}\right. \\
& \left.+\frac{1}{2}\left(w_{, y}+v_{, z}\right) \operatorname{sen}\left(2 \phi_{0}\right)\right]+\left(\alpha_{3} \operatorname{sen}^{2} \phi_{0}-\alpha_{2} \cos ^{2} \phi_{0}\right)\left[\frac{1}{2}\left(v_{, z}-w_{, y}\right)\right] \\
& +\frac{1}{2}\left(\alpha_{5} \cos ^{2} \phi_{0}+\alpha_{6} \operatorname{sen}^{2} \phi_{0}\right)\left(w_{, y}+v_{, z}\right)+\operatorname{sen} \phi_{0} \cos \phi_{0}\left(\alpha_{5} v_{, y}+\alpha_{6} w_{, z}\right) \text {, } \\
& \Phi_{z x}=\frac{1}{2} \alpha_{3}\left[\cos ^{2} \phi_{0}\left(w_{, x}-u_{, z}\right)+\operatorname{sen} \phi_{0} \cos \phi_{0}\left(v_{, x}-u_{, y}\right)\right] \\
& +\frac{1}{2} \alpha_{6}\left[\cos ^{2} \phi_{0}\left(u_{, z}+w_{, x}\right)+\operatorname{sen} \phi_{0} \cos \phi_{0}\left(v_{, x}+u_{, y}\right)\right], \\
& \Phi_{z y}=\alpha_{1} \operatorname{sen} \phi_{0} \cos \phi_{0}\left[w_{, z} \cos ^{2} \phi_{0}+v_{, y} \operatorname{sen}^{2} \phi_{0}\right. \\
& \left.+\frac{1}{2}\left(w_{, y}+v_{, z}\right) \operatorname{sen}\left(2 \phi_{0}\right)\right]+\left(\alpha_{2} \operatorname{sen}^{2} \phi_{0}-\alpha_{3} \cos ^{2} \phi_{0}\right)\left[\frac{1}{2}\left(v_{, z}-w_{, y}\right)\right] \\
& +\frac{1}{2}\left(\alpha_{5} \operatorname{sen}^{2} \phi_{0}+\alpha_{6} \cos ^{2} \phi_{0}\right)\left(w_{, y}+v_{, z}\right)+\operatorname{sen} \phi_{0} \cos \phi_{0}\left(\alpha_{5} w_{, z}+\alpha_{6} v_{, y}\right) \text {, } \\
& \Phi_{z z}=\alpha_{1} \cos ^{2} \phi_{0}\left[w_{, z} \cos ^{2} \phi_{0}+v_{, y} \operatorname{sen}^{2} \phi_{0}+\frac{1}{2}\left(w_{, y}+v_{, z}\right) \operatorname{sen}\left(2 \phi_{0}\right)\right] \\
& -\left(\alpha_{2}+\alpha_{3}\right) \operatorname{sen} \phi_{0} \cos \phi_{0}\left[\frac{1}{2}\left(w_{, y}-v_{, z}\right)\right] \\
& +\left(\alpha_{5}+\alpha_{6}\right)\left[w_{, z} \cos ^{2} \phi_{0}+\frac{1}{2} \operatorname{sen} \phi_{0} \cos \phi_{0}\left(w_{, y}+v_{, z}\right)\right] .
\end{aligned}
$$

Nas equações acima, as viscosidades $\alpha_{1}, \ldots, \alpha_{6}$ foram escalonadas pelo fator $\eta$. 
Portanto, as equações de conservação de quantidade de movimento (2.34)-(2.36) podem ser escritas na forma

$$
\begin{aligned}
u_{t}+ & (u u)_{, x}+(v u)_{, y}+(w u)_{, z}=-p_{, x} \\
& +\frac{1}{R e}\left[u_{, x x}+u_{, y y}+u_{, z z}+\Phi_{x x, x}+\Phi_{x y, y}+\Phi_{x z, z}\right], \\
v_{t}+ & (u v)_{, x}+(v v)_{, y}+(w v)_{, z}=-p_{, y} \\
+ & +\frac{1}{R e}\left[v_{, x x}+v_{, y y}+v_{, z z}+\Phi_{y x, x}+\Phi_{y y, y}+\Phi_{y z, z}\right], \\
w_{t}+ & (u w)_{, x}+(v w)_{, y}+(w w)_{, z}=-p_{, z} \\
& +\frac{1}{R e}\left[w_{, x x}+w_{, y y}+w_{, z z}+\Phi_{z x, x}+\Phi_{z y, y}+\Phi_{z z, z}\right]
\end{aligned}
$$

e a equação de conservação de massa (2.37) permanece inalterada.

Portanto, para simular o escoamento de um cristal líquido nemático sob um forte campo magnético, temos que resolver as equações (2.56)-(2.58) e (2.37) juntamente com as equações (2.47)-(2.55) para as variáveis $u, v, w, p$ e as componentes do tensor das tensões não-newtoniano $\Phi_{x x}, \cdots, \Phi_{z z}$, respectivamente.

\subsection{Condições de Contorno}

Para resolver as equações $(2.37),(2.56)-(2.58)$, é necessário impor condições de contorno para o campo de velocidade, como segue.

- Em contornos rígidos emprega-se a condição de não-deslizamento $u_{i}=0$.

- Em entradas e saídas de fluido, os valores da velocidade são especificados como segue:

i) $\mathrm{Na}$ entrada de fluido ('inflows') a velocidade normal é prescrita como $u_{\nu}=U_{\text {inf }}$ enquanto que as velocidades tangenciais são nulas, ou seja, $u_{\mu_{1}}=u_{\mu_{2}}=0$, onde $\mu_{1}$ e $\mu_{2}$ denotam direções tangenciais ao inflow.

ii) Na saída de fluido ('outflow') considera-se que não há variação da velocidade e então impõe-se a condição homogênea de Neumann $u_{i, \nu}=0$.

\subsection{Método de Solução}

O método GENSMAC3D, descrito por Tomé et. al. [127], faz parte de um grupo de métodos numéricos que utilizam a estratégia do desacoplamento dos campos de velocidade e pressão das equações (2.37), (2.56)-(2.58). Esses métodos foram, originalmente, propostos por Harlow e Welch [68], Chorin [27, 28], e Temam [125] e são conhecidos como "métodos de projeção" 
ou "métodos de passo fracionário". A ideia básica da metodologia GENSMAC3D é utilizar as equações de conservação de quantidade de movimento (2.56)-(2.58) para calcular um campo de velocidade intermediária, que geralmente não satisfaz a equação de conservação de massa (2.37), por meio da equação

$$
\frac{\partial \widetilde{u}_{i}}{\partial t}+\left(u_{j} u_{i}\right), j_{j}=\frac{1}{R e}\left(\left(u_{i, j}\right),_{j}+\Phi_{i j, j}\right)
$$

Pelo Teorema da Decomposição de Helmholtz-Hodge [71], existe uma função escalar $\psi$ tal que

$$
\widetilde{u}_{i}=u_{i}+\psi_{, i}
$$

em que $u_{i, i}=0$. Aplicando o divergente em ambos os lados da equação obtém-se

$$
\widetilde{u}_{i, i}=\psi_{, i i} .
$$

Logo, a função escalar $\psi$ é obtida pela solução de (2.61) e a velocidade $u_{i}$ é calculada pela equação (2.60). A principal vantagem dos métodos tipo projeção é que os cálculos dos campos de velocidade e pressão são desacoplados. Dessa forma, a solução das equações (2.37), (2.56)-(2.58), é obtida como segue.

Assume-se que, no instante de tempo $t_{n}$, o campo de velocidade $u_{i}\left(x_{k}, t_{n}\right)$ é conhecido e que as condições de contorno para a velocidade e pressão são dadas. A velocidade $u_{i}\left(x_{k}, t_{n+1}\right)$, a pressão $p\left(x_{k}, t_{n+1}\right)$ e o tensor não newtoniano $\Phi_{i j}\left(x_{k}, t_{n+1}\right)$ são obtidos pelo seguinte procedimento.

Passo 1: Utilizando os valores de $u_{i}\left(x_{k}, t_{n}\right)$ calcula-se $\Phi_{i j}\left(x_{k}, t_{n}\right)$ utilizando as equações (2.47)-(2.55).

Passo 2: Calcula-se o campo de velocidade intermediária $\widetilde{u}_{i}\left(x_{k}, t_{n+1}\right)$ por meio da equação $(2.59)$.

Passo 3: Resolve-se a equação de Poisson (2.61) para $\psi\left(x_{k}, t_{n+1}\right)$. As condições de contorno para essa equação são: $\psi_{, \nu}=0$ em contornos rígidos e na entrada de fluido e $\psi=0$ nas saídas de fluido (para detalhes ver Tomé et al. [127]).

Passo 4: Obtém-se o campo de velocidades final, $u_{i}\left(x_{k}, t_{n+1}\right)$, por $(2.60)$.

Passo 5: Calcula-se a pressão. Pode ser mostrado que a pressão é dada por (ver Tomé et al. [131])

$$
p\left(x_{k}, t_{n+1}\right)=\frac{\psi\left(x_{k}, t_{n+1}\right)}{\delta t} .
$$

Passo 6: Calcula-se as componentes do tensor não-newtoniano $\Phi_{i j}\left(x_{k}, t_{n+1}\right)$ através das equações $(2.47)-(2.55)$. 


\subsection{Aproximação das Equações por Diferenças Finitas}

As equações que descrevem o método numérico apresentado na Seção 2.6 são resolvidas pela técnica de diferenças finitas em uma malha deslocada tridimensional. Este tipo de malha foi inicialmente introduzida por Harlow e Welch [68] e tem sido utilizada com o método MAC (Marker and Cell) por ser apropriada para o uso de métodos de projeção e computacionalmente simples.

As velocidades $u, v, w$ são localizadas nas faces da célula enquanto que as quantidades escalares e demais grandezas são posicionadas no centro da célula. A Figura 2.2 ilustra uma célula tridimensional com lados de comprimento $\delta x, \delta y, \delta z$.

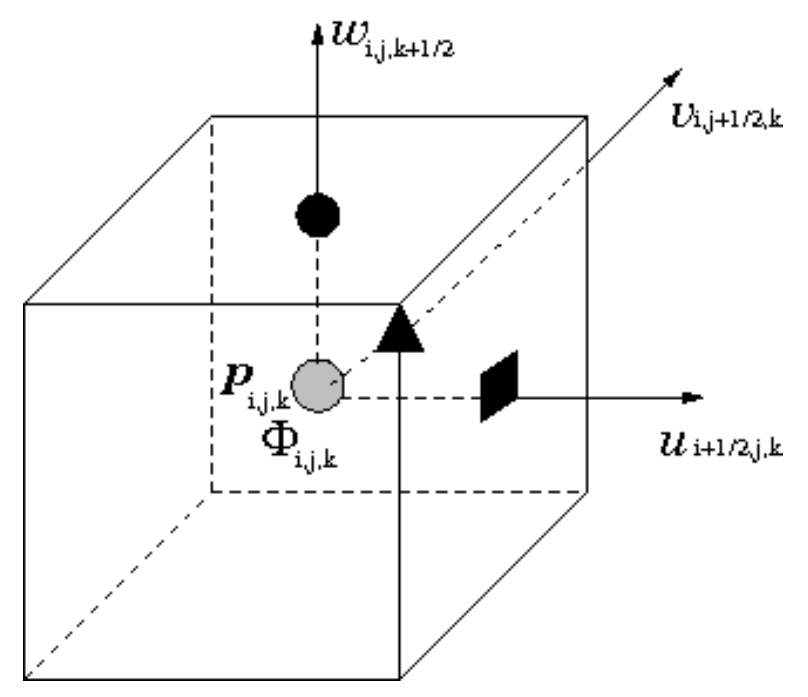

Figura 2.2: Típica célula tridimensional.

No cálculo das componentes da velocidade intermediária $\widetilde{u}_{i}$, as derivadas temporais nas equações (2.56)-(2.58) são aproximadas pelo método de Euler explícito enquanto que as derivadas espaciais são aproximadas por diferenças finitas de segunda ordem.

Em resumo, as equações de conservação de quantidade de movimento (2.56)-(2.58) são aproximadas pelas equações

$$
\begin{aligned}
\widetilde{u}_{i+1 / 2, j, k} & =u_{i+1 / 2, j, k}+\delta t\left\{-\left.\mathcal{C}(u)\right|_{i+1 / 2, j, k}+\frac{1}{R e}\left[\left(\frac{u_{i-1 / 2, j, k}-2 u_{i+1 / 2, j, k}+u_{i+3 / 2, j, k}}{\delta x^{2}}\right)\right.\right. \\
& +\left(\frac{u_{i+1 / 2, j-1, k}-2 u_{i+1 / 2, j, k}+u_{i+1 / 2, j+1, k}}{\delta y^{2}}\right)+\left(\frac{u_{i+1 / 2, j, k-1}-2 u_{i+1 / 2, j, k}+u_{i+1 / 2, j, k+1}}{\delta z^{2}}\right) \\
& +\left(\frac{\Phi_{i+1, j, k}^{x x}-\Phi_{i, j, k}^{x x}}{\delta x}\right)+\left(\frac{\Phi_{i+1 / 2, j+1 / 2, k}^{x y}-\Phi_{i+1 / 2, j-1 / 2, k}^{x y}}{\delta y}\right) \\
& \left.\left.+\left(\frac{\Phi_{i+1 / 2, j, k+1 / 2}^{x z}-\Phi_{i+1 / 2, j, k-1 / 2}^{x z}}{\delta z}\right)\right]\right\}
\end{aligned}
$$




$$
\begin{aligned}
\widetilde{v}_{i, j+1 / 2, k} & =v_{i, j+1 / 2, k}+\delta t\left\{-\left.\mathcal{C}(v)\right|_{i, j+1 / 2, k}+\frac{1}{R e}\left[\left(\frac{v_{i-1, j+1 / 2, k}-2 v_{i, j+1 / 2, k}+v_{i+1, j+1 / 2, k}}{\delta x^{2}}\right)\right.\right. \\
& +\left(\frac{v_{i, j-1 / 2, k}-2 v_{i, j+1 / 2, k}+v_{i, j+3 / 2, k}}{\delta y^{2}}\right)+\left(\frac{v_{i, j+1 / 2, k-1}-2 v_{i, j+1 / 2, k}+v_{i, j+1 / 2, k+1}}{\delta z^{2}}\right) \\
& +\left(\frac{\Phi_{i+1 / 2, j+1 / 2, k}^{y x}-\Phi_{i-1 / 2, j+1 / 2, k}^{y x}}{\delta x}\right) \\
& +\left(\frac{\Phi_{i, j+1, k}^{y y}-\Phi_{i, j, k}^{y y}}{\delta y}+\left(\frac{\Phi_{i, j+1 / 2, k+1 / 2}^{y z}-\Phi_{i, j+1 / 2, k-1 / 2}^{y z}}{\delta z}\right)\right], \\
\widetilde{w}_{i, j, k+1 / 2} & =w_{i, j, k+1 / 2}+\delta t\left\{-\left.\mathcal{C}(w)\right|_{i, j, k+1 / 2}+\frac{1}{R e}\left[\left(\frac{w_{i-1, j, k+1 / 2}-2 w_{i, j, k+1 / 2}+w_{i+1, j, k+1 / 2}}{\delta x^{2}}\right)\right.\right. \\
& +\left(\frac{w_{i, j-1, k+1 / 2}-2 w_{i, j, k+1 / 2}+w_{i, j+1, k+1 / 2}}{\delta y^{2}}\right)+\left(\frac{w_{i, j, k-1 / 2}-2 w_{i, j, k+1 / 2}+w_{i, j, k+3 / 2}}{\delta z^{2}}\right) \\
& +\left(\frac{\Phi_{i+1 / 2, j, k+1 / 2}^{z x}-\Phi_{i-1 / 2, j, k+1 / 2}^{z x}}{\delta x}\right) \\
& \left.+\left(\frac{\Phi_{i, j+1 / 2, k+1 / 2}^{z y}-\Phi_{i, j-1 / 2, k+1 / 2}^{z y}}{\delta y}\left(\frac{\Phi_{i, j, k+1}^{z z}-\Phi_{i, j, k}^{z z}}{\delta z}\right)\right]\right\}
\end{aligned}
$$

em que $\Phi_{i, j, k}^{l m}$ são as componentes do tensor das tensões não-newtoniano e são tratados na próxima Seção.

Os termos $\mathcal{C}(u), \mathcal{C}(v), \mathcal{C}(w)$ nas equações (2.63)-(2.65), representam os termos convectivos:

$$
\begin{aligned}
\mathcal{C}(u) & =(u u)_{, x}+(v u)_{, y}+(w u)_{, z} \\
\mathcal{C}(v) & =(u v)_{, x}+(v v)_{, y}+(w v)_{, z} \\
\mathcal{C}(w) & =(u w)_{, x}+(v w)_{, y}+(w w)_{, z}
\end{aligned}
$$

e $\left.\mathcal{C}(u)\right|_{i+1 / 2, j, k},\left.\mathcal{C}(v)\right|_{i, j+1 / 2, k}$ e $\left.\mathcal{C}(w)\right|_{i, j, k+1 / 2}$ representam uma discretização dos termos convectivos utilizando o método 'upwind' de alta ordem CUBISTA (ver Alves et al. [1]). Detalhes específicos da discretização fornecida pelo método CUBISTA pode ser encontrado em Ferreira et al. [50].

Nas equações acima, termos tais como $\Phi_{i+1 / 2, j+1 / 2, k}^{x y}$ e $\Phi_{i, j+1 / 2, k+1 / 2}^{y z}$ que não são definidos em seus respectivos nós são obtidos pela média aritmética dos nós vizinhos como segue:

$$
\begin{aligned}
\Phi_{i+1 / 2, j+1 / 2, k}^{x y} & =\frac{\Phi_{i, j, k}^{x y}+\Phi_{i+1, j, k}^{x y}+\Phi_{i, j+1, k}^{x y}+\Phi_{i+1, j+1, k}^{x y}}{4}, \\
\Phi_{i, j+1 / 2, k+1 / 2}^{y z} & =\frac{\Phi_{i, j, k}^{y z}+\Phi_{i, j+1, k}^{y z}+\Phi_{i, j, k+1}^{y z}+\Phi_{i, j+1, k+1}^{y z}}{4} .
\end{aligned}
$$

A equação de Poisson dada por (2.62) é discretizada no centro da célula utilizando o operador 
Laplaciano fornecendo a seguinte equação de diferenças:

$\frac{\psi_{i+1, j, k}-2 \psi_{i, j, k}+\psi_{i-1, j, k}}{\delta x^{2}}+\frac{\psi_{i, j+1, k}-2 \psi_{i, j, k}+\psi_{i, j-1, k}}{\delta y^{2}}+\frac{\psi_{i, j, k+1}-2 \psi_{i, j, k}+\psi_{i, j, k-1}}{\delta z^{2}}=\tilde{D}_{i, j, k}$

onde

$$
\tilde{D}_{i, j, k}=\frac{\tilde{u}_{i+1 / 2, j, k}-\tilde{u}_{i-1 / 2, j, k}}{\delta x}+\frac{\tilde{v}_{i, j+1 / 2, k}-\tilde{v}_{i, j-1 / 2, k}}{\delta y}+\frac{\tilde{w}_{i, j, k+1 / 2}-\tilde{w}_{i, j, k-1 / 2}}{\delta z} .
$$

A equação (2.66) resulta em um sistema linear de equações para $\psi_{i, j}$, cuja matriz associada é simétrica e positiva definida. Este sistema resultante é então resolvido eficientemente (sem a necessidade de pré-condicionadores) pelo método dos gradientes conjugados. As componentes da velocidade $u, v$, e $w$ no tempo $t_{n+1}$ são então obtidas pela discretização da equação (2.60) em seus respectivos nós:

$$
\begin{aligned}
& u_{i+1 / 2, j, k}^{(n+1)}=\tilde{u}_{i+1 / 2, j, k}-\left(\frac{\psi_{i+1, j, k}-\psi_{i, j, k}}{\delta x}\right), \\
& v_{i, j+1 / 2, k}^{(n+1)}=\tilde{v}_{i, j+1 / 2, k}-\left(\frac{\psi_{i, j+1, k}-\psi_{i, j, k}}{\delta y}\right), \\
& w_{i, j, k+1 / 2}^{(n+1)}=\tilde{w}_{i, j, k+1 / 2}-\left(\frac{\psi_{i, j, k+1}-\psi_{i, j, k}}{\delta z}\right) .
\end{aligned}
$$

A pressão é calculada por

$$
p_{i, j, k}=\frac{\psi_{i, j, k}}{\delta t}
$$

\subsubsection{Cálculo das Componentes do Tensor das Tensões não-newtoniano}

As componentes do tensor das tensões não-newtoniano dado pelas equações (2.47)-(2.55) são aproximadas por diferenças centrais. Os termos envolvendo derivadas cruzadas como por exemplo $u_{, y}$ e $v_{, x}$ são obtidos aproximados por:

$$
\left.u_{, y}\right|_{i, j, k}=\frac{u_{i, j+1 / 2, k}-u_{i, j-1 / 2, k}}{\delta y},\left.\quad v_{, x}\right|_{i, j, k}=\frac{v_{i+1 / 2, j, k}-v_{i-1 / 2, j, k}}{\delta x}
$$

em que

$$
\begin{aligned}
& u_{i, j+1 / 2, k}=0.25\left(u_{i+1 / 2, j, k}+u_{i-1 / 2, j, k}+u_{i+1 / 2, j+1, k}+u_{i-1 / 2, j+1, k}\right) \\
& u_{i, j-1 / 2, k}=0.25\left(u_{i+1 / 2, j, k}+u_{i-1 / 2, j, k}+u_{i+1 / 2, j-1, k}+u_{i-1 / 2, j-1, k}\right) \\
& v_{i+1 / 2, j, k}=0.25\left(v_{i, j+1 / 2, k}+v_{i, j-1 / 2, k}+v_{i+1, j+1 / 2, k}+v_{i+1, j-1 / 2, k}\right) \\
& v_{i-1 / 2, j, k}=0.25\left(v_{i, j+1 / 2, k}+v_{i, j-1 / 2, k}+v_{i-1, j+1 / 2, k}+v_{i-1, j-1 / 2, k}\right) .
\end{aligned}
$$


As derivadas $\left.u_{, x}\right|_{i, j, k},\left.v_{, y}\right|_{i, j, k}$ e $\left.w_{, z}\right|_{i, j, k}$ são facilmente aproximadas por diferenças finitas de 2a. ordem. Logo, as equações para as componentes do tensor das tensões não-newtoniano (2.47)-(2.55) são aproximados por

$$
\begin{aligned}
\Phi_{i, j, k}^{x x} & =0 \\
\Phi_{i, j, k}^{x y} & =\frac{1}{2} \alpha_{2}\left[\operatorname{sen}^{2} \phi_{0}\left(\frac{v_{i+1 / 2, j, k}-v_{i-1 / 2, j, k}}{\delta x}-\frac{u_{i, j+1 / 2, k}-u_{i, j-1 / 2, k}}{\delta y}\right)\right. \\
& \left.+\operatorname{sen} \phi_{0} \cos \phi_{0}\left(\frac{w_{i+1 / 2, j, k}-w_{i-1 / 2, j, k}}{\delta x}-\frac{u_{i, j, k+1 / 2}-u_{i, j, k-1 / 2}}{\delta z}\right)\right] \\
& +\frac{1}{2} \alpha_{5}\left[\operatorname{sen}^{2} \phi_{0}\left(\frac{v_{i+1 / 2, j, k}-v_{i-1 / 2, j, k}}{\delta x}+\frac{u_{i, j+1 / 2, k}-u_{i, j-1 / 2, k}}{\delta y}\right)\right. \\
& \left.+\operatorname{sen} \phi_{0} \cos \phi_{0}\left(\frac{u_{i, j, k+1 / 2}-u_{i, j, k-1 / 2}}{\delta z}+\frac{w_{i+1 / 2, j, k}-w_{i-1 / 2, j, k}}{\delta x}\right)\right]
\end{aligned}
$$

$$
\begin{aligned}
\Phi_{i, j, k}^{x z} & =\frac{1}{2} \alpha_{2}\left[\cos ^{2} \phi_{0}\left(\frac{w_{i+1 / 2, j, k}-w_{i-1 / 2, j, k}}{\delta x}-\frac{u_{i, j, k+1 / 2}-u_{i, j, k-1 / 2}}{\delta z}\right)\right. \\
& \left.+\operatorname{sen} \phi_{0} \cos \phi_{0}\left(\frac{v_{i+1 / 2, j, k}-v_{i-1 / 2, j, k}}{\delta x}-\frac{u_{i, j+1 / 2, k}-u_{i, j-1 / 2, k}}{\delta y}\right)\right] \\
& +\frac{1}{2} \alpha_{5}\left[\cos ^{2} \phi_{0}\left(\frac{u_{i, j, k+1 / 2}-u_{i, j, k-1 / 2}}{\delta z}+\frac{w_{i+1 / 2, j, k}-w_{i-1 / 2, j, k}}{\delta x}\right)\right. \\
& \left.+\operatorname{sen} \phi_{0} \cos \phi_{0}\left(\frac{v_{i+1 / 2, j, k}-v_{i-1 / 2, j, k}}{\delta x}+\frac{u_{i, j+1 / 2, k}-u_{i, j-1 / 2, k}}{\delta y}\right)\right]
\end{aligned}
$$

$$
\begin{aligned}
\Phi_{i, j, k}^{y x} & =\frac{1}{2} \alpha_{3}\left[\operatorname{sen}^{2} \phi_{0}\left(\frac{v_{i+1 / 2, j, k}-v_{i-1 / 2, j, k}}{\delta x}-\frac{u_{i, j+1 / 2, k}-u_{i, j-1 / 2, k}}{\delta y}\right)\right. \\
& \left.+\operatorname{sen} \phi_{0} \cos \phi_{0}\left(\frac{w_{i+1 / 2, j, k}-w_{i-1 / 2, j, k}}{\delta x}-\frac{u_{i, j, k+1 / 2}-u_{i, j, k-1 / 2}}{\delta z}\right)\right] \\
& +\frac{1}{2} \alpha_{6}\left[\operatorname{sen}^{2} \phi_{0}\left(\frac{v_{i+1 / 2, j, k}-v_{i-1 / 2, j, k}}{\delta x}+\frac{u_{i, j+1 / 2, k}-u_{i, j-1 / 2, k}}{\delta y}\right)\right. \\
& \left.+\operatorname{sen} \phi_{0} \cos \phi_{0}\left(\frac{u_{i, j, k+1 / 2}-u_{i, j, k-1 / 2}}{\delta z}+\frac{w_{i+1 / 2, j, k}-w_{i-1 / 2, j, k}}{\delta x}\right)\right],
\end{aligned}
$$




$$
\begin{aligned}
\Phi_{i, j, k}^{y y} & =\alpha_{1} \operatorname{sen}^{2} \phi_{0}\left[\frac{w_{i, j, k+1 / 2}-w_{i, j, k-1 / 2}}{\delta z} \cos ^{2} \phi_{0}+\frac{v_{i, j+1 / 2, k}-v_{i, j-1 / 2, k}}{\delta y} \operatorname{sen}^{2} \phi_{0}\right. \\
& \left.+\frac{1}{2}\left(\frac{w_{i, j+1 / 2, k}-w_{i, j-1 / 2, k}}{\delta y}+\frac{v_{i, j, k+1 / 2}-v_{i, j, k-1 / 2}}{\delta z}\right) \operatorname{sen}\left(2 \phi_{0}\right)\right] \\
& +\left(\alpha_{2}+\alpha_{3}\right) \operatorname{sen} \phi_{0} \cos \phi_{0}\left[\frac{1}{2}\left(\frac{w_{i, j+1 / 2, k}-w_{i, j-1 / 2, k}}{\delta y}-\frac{v_{i, j, k+1 / 2}-v_{i, j, k-1 / 2}}{\delta z}\right)\right] \\
& +\left(\alpha_{5}+\alpha_{6}\right)\left[\frac{v_{i, j+1 / 2, k}-v_{i, j-1 / 2, k}}{\delta y} \operatorname{sen}^{2} \phi_{0}+\frac{1}{2} \operatorname{sen} \phi_{0} \cos \phi_{0}\left(\frac{w_{i, j+1 / 2, k}-w_{i, j-1 / 2, k}}{\delta y}\right.\right. \\
& \left.\left.+\frac{v_{i, j, k+1 / 2}-v_{i, j, k-1 / 2}}{\delta z}\right)\right]
\end{aligned}
$$

$$
\begin{aligned}
\Phi_{i, j, k}^{y z} & =\alpha_{1} \operatorname{sen} \phi_{0} \cos \phi_{0}\left[\frac{w_{i, j, k+1 / 2}-w_{i, j, k-1 / 2}}{\delta z} \cos ^{2} \phi_{0}+\frac{v_{i, j+1 / 2, k}-v_{i, j-1 / 2, k}}{\delta y} \operatorname{sen}^{2} \phi_{0}\right. \\
& \left.+\frac{1}{2}\left(\frac{w_{i, j+1 / 2, k}-w_{i, j-1 / 2, k}}{\delta y}+\frac{v_{i, j, k+1 / 2}-v_{i, j, k-1 / 2}}{\delta z}\right) \operatorname{sen}\left(2 \phi_{0}\right)\right] \\
& +\operatorname{sen} \phi_{0} \cos \phi_{0}\left(\alpha_{5} \frac{v_{i, j+1 / 2, k}-v_{i, j-1 / 2, k}}{\delta y}+\alpha_{6} \frac{w_{i, j, k+1 / 2}-w_{i, j, k-1 / 2}}{\delta z}\right) \\
& +\frac{1}{2}\left(\alpha_{5} \cos ^{2} \phi_{0}+\alpha_{6} \operatorname{sen}^{2} \phi_{0}\right)\left(\frac{w_{i, j+1 / 2, k}-w_{i, j-1 / 2, k}}{\delta y}+\frac{v_{i, j, k+1 / 2}-v_{i, j, k-1 / 2}}{\delta z}\right) \\
& +\left(\alpha_{3} \operatorname{sen}^{2} \phi_{0} \alpha_{2} \cos ^{2} \phi_{0}\right)\left[\frac{1}{2}\left(\frac{v_{i, j, k+1 / 2}-v_{i, j, k-1 / 2}}{\delta z}-\frac{w_{i, j+1 / 2, k}-w_{i, j-1 / 2, k}}{\delta y}\right)\right]
\end{aligned}
$$

$$
\begin{aligned}
\Phi_{i, j, k}^{z x} & =\alpha_{1} \operatorname{sen} \phi_{0} \cos \phi_{0}\left[\frac{w_{i, j, k+1 / 2}-w_{i, j, k-1 / 2}}{\delta z} \cos ^{2} \phi_{0}+\frac{v_{i, j+1 / 2, k}-v_{i, j-1 / 2, k}}{\delta y} \operatorname{sen}^{2} \phi_{0}\right. \\
& \left.+\frac{1}{2}\left(\frac{w_{i, j+1 / 2, k}-w_{i, j-1 / 2, k}}{\delta y}+\frac{v_{i, j, k+1 / 2}-v_{i, j, k-1 / 2}}{\delta z}\right) \operatorname{sen}\left(2 \phi_{0}\right)\right] \\
& +\frac{1}{2}\left(\alpha_{5} \cos ^{2} \phi_{0}+\alpha_{6} \operatorname{sen}^{2} \phi_{0}\right)\left(\frac{w_{i, j+1 / 2, k}-w_{i, j-1 / 2, k}}{\delta y}+\frac{v_{i, j, k+1 / 2}-v_{i, j, k-1 / 2}}{\delta z}\right) \\
& +\operatorname{sen} \phi_{0} \cos \phi_{0}\left(\alpha_{5} \frac{v_{i, j+1 / 2, k}-v_{i, j-1 / 2, k}}{\delta y}+\alpha_{6} \frac{w_{i, j, k+1 / 2}-w_{i, j, k-1 / 2}}{\delta z}\right) \\
& +\frac{1}{2}\left(\alpha_{3} \operatorname{sen}^{2} \phi_{0}-\alpha_{2} \cos ^{2} \phi_{0}\right)\left[\left(\frac{v_{i, j, k+1 / 2}-v_{i, j, k-1 / 2}}{\delta z}-\frac{w_{i, j+1 / 2, k}-w_{i, j-1 / 2, k}}{\delta y}\right)\right],
\end{aligned}
$$




$$
\begin{aligned}
\Phi_{i, j, k}^{z y} & =\alpha_{1} \operatorname{sen} \phi_{0} \cos \phi_{0}\left[\frac{w_{i, j, k+1 / 2}-w_{i, j, k-1 / 2}}{\delta z} \cos ^{2} \phi_{0}+\frac{v_{i, j+1 / 2, k}-v_{i, j-1 / 2, k}}{\delta y} \operatorname{sen}^{2} \phi_{0}\right. \\
& \left.+\frac{1}{2}\left(\frac{w_{i, j+1 / 2, k}-w_{i, j-1 / 2, k}}{\delta y}+\frac{v_{i, j, k+1 / 2}-v_{i, j, k-1 / 2}}{\delta z}\right) \operatorname{sen}\left(2 \phi_{0}\right)\right] \\
& +\frac{1}{2}\left(\alpha_{5} \operatorname{sen}^{2} \phi_{0}+\alpha_{6} \cos ^{2} \phi_{0}\right)\left(\frac{w_{i, j+1 / 2, k}-w_{i, j-1 / 2, k}}{\delta y}+\frac{v_{i, j, k+1 / 2}-v_{i, j, k-1 / 2}}{\delta z}\right) \\
& +\operatorname{sen} \phi_{0} \cos \phi_{0}\left(\alpha_{5} \frac{w_{i, j, k+1 / 2}-w_{i, j, k-1 / 2}}{\delta z}+\alpha_{6} \frac{v_{i, j+1 / 2, k}-v_{i, j-1 / 2, k}}{\delta y}\right) \\
& +\frac{1}{2}\left(\alpha_{2} \operatorname{sen}^{2} \phi_{0}-\alpha_{3} \cos ^{2} \phi_{0}\right)\left[\left(\frac{v_{i, j, k+1 / 2}-v_{i, j, k-1 / 2}}{\delta z}-\frac{w_{i, j+1 / 2, k}-w_{i, j-1 / 2, k}}{\delta y}\right)\right] \\
\Phi_{i, j, k}^{z z} & =\alpha_{1} \cos ^{2} \phi_{0}\left[\frac{w_{i, j, k+1 / 2}-w_{i, j, k-1 / 2} \cos ^{2} \phi_{0}+\frac{v_{i, j+1 / 2, k}-v_{i, j-1 / 2, k}}{\delta z} \operatorname{sen}^{2} \phi_{0}}{\delta y}\right) \\
& \left.+\frac{1}{2}\left(\frac{w_{i, j+1 / 2, k}-w_{i, j-1 / 2, k}}{\delta y}+\frac{v_{i, j, k+1 / 2}-v_{i, j, k-1 / 2}}{\delta z}\right) \operatorname{sen}\left(2 \phi_{0}\right)\right] \\
& -\left(\alpha_{2}+\alpha_{3}\right) \operatorname{sen} \phi_{0} \cos \phi_{0}\left[\frac{1}{2}\left(\frac{w_{i, j+1 / 2, k}-w_{i, j-1 / 2, k}}{\delta y}\right)\right] \\
& \left.-\frac{v_{i, j, k+1 / 2}-v_{i, j, k-1 / 2}}{\delta z}\right)\left(\alpha_{5}+\alpha_{6}\right)\left[\frac{w_{i, j, k+1 / 2}-w_{i, j, k-1 / 2}}{\delta z} \cos ^{2} \phi_{0}\right. \\
+ & \left.\frac{1}{2} \operatorname{sen} \phi_{0} \cos \phi_{0}\left(\frac{w_{i, j+1 / 2, k}-w_{i, j-1 / 2, k}}{\delta y}+\frac{v_{i, j, k+1 / 2}-v_{i, j, k-1 / 2}}{\delta z}\right)\right]
\end{aligned}
$$

\subsubsection{Cálculo do Passo Temporal}

Devido as restrições de estabilidade, a aproximação explícita das equações de conservação de quantidade de movimento pode conduzir a tamanhos de passo no tempo muito pequenos o que pode resultar em um código computacionalmente ineficiente. Com o objetivo otimizar essas restrições, um passo temporal é escolhido de modo a ser o maior possível e satisfazendo as condições de estabilidade. Assim, o tamanho do passo temporal é obtido de modo a satisfazer as seguintes condições de estabilidade:

$$
\begin{aligned}
\delta t_{x} & \leq \frac{\delta x}{|u|_{\max }} \\
\delta t_{y} & \leq \frac{\delta y}{|v|_{\max }} \\
\delta t_{z} & \leq \frac{\delta z}{|w|_{\max }}
\end{aligned}
$$

onde $|u|_{\text {max }},|v|_{\text {max }}$ e $|w|_{\text {max }}$ são os módulos máximos das velocidades nas direções $x, y$ e $z$, respectivamente. Estas restrições são conhecidas na literatura como "condição Courant-FriedrichsLewy (CFL)" [31]. Por outro lado, a discretização explícita da equação de conservação de 
quantidade de movimento também impõe uma restrição de estabilidade. Essa restrição, no caso tridimensional é dada pela expressão

$$
\delta t_{v i s c} \leq \frac{R e}{2}\left(\frac{1}{(\delta x)^{2}}+\frac{1}{(\delta y)^{2}}+\frac{1}{(\delta z)^{2}}\right)^{-1} .
$$

A implementação dessas restrições para determinar o tamanho do passo temporal admissível é baseado nas ideias de Tomé e McKee [134]. Detalhes precisos sobre a implementação do cálculo do passo temporal pode ser encontrado em Tomé et al. [127].

\subsection{Verificação do Método Numérico}

As equações de diferenças finitas correspondentes ao método numérico apresentado nesse Capítulo foram incorporadas ao sistema Freeflow3D [22] para simular escoamentos tridimensionais de cristais líquidos nemáticos sob efeito de um forte campo magnético. Para validar a implementação desse método numérico, o escoamento entre duas placas paralelas será simulado e os resultados numéricos serão comparados com a solução analítica para escoamentos totalmente desenvolvidos entre dua placas paralelas.

\subsubsection{Solução Analítica do Escoamento entre Duas Placas}

A solução analítica do escoamento entre duas placas sujeitas a um forte campo magnético ((2.4), (2.5) e (2.8)) foi apresentada na Seção 2.1 e detalhes sobre a obtenção dessa solução analítica são encontrados em [123].

O ângulo de deflexão $\varphi$ é mostrado na Figura 2.3 e pode ser obtido da seguinte relação

$$
\tan \varphi=\frac{v}{w}=-\frac{\left(\alpha_{3}+\alpha_{6}\right) \operatorname{sen} \phi_{0} \cos \phi_{0}}{\alpha_{4}+\left(\alpha_{3}+\alpha_{6}\right) \operatorname{sen}^{2} \phi_{0}}
$$

ou

$$
\tan \varphi=\frac{\left(\eta_{3}-\eta_{1}\right) \operatorname{sen} \phi_{0} \cos \phi_{0}}{\eta_{1} \operatorname{sen}^{2} \phi_{0}+\eta_{3} \cos ^{2} \phi_{0}}
$$

utilizando (2.6) e (2.7). As soluções analíticas das componentes do tensor não-newtoniano $\Phi_{i j}$ 


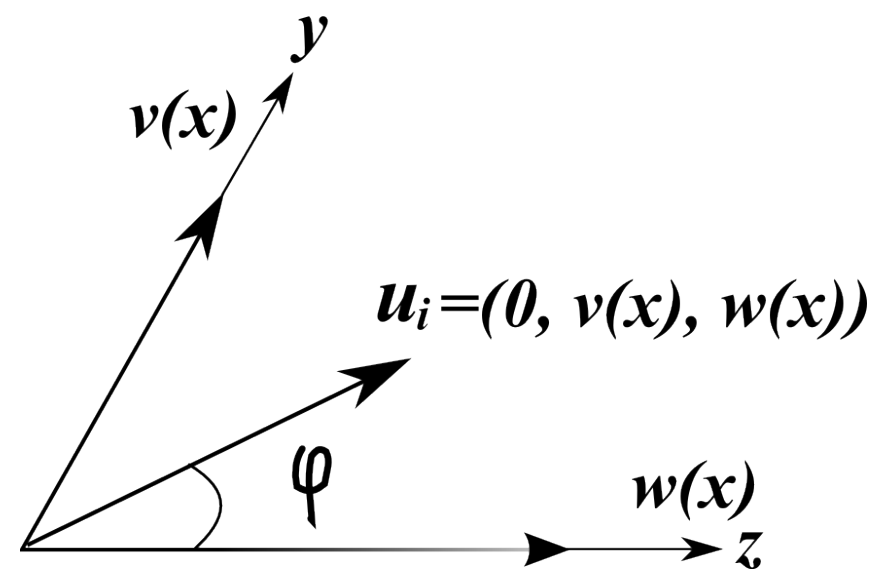

Figura 2.3: Representação do ângulo de deflexão $\varphi$.

são obtidas das equações (2.47)-(2.55) que reduzem-se à

$$
\begin{aligned}
\Phi^{x x} & =0 \\
\Phi^{x y} & =\frac{1}{2}\left(\alpha_{2}+\alpha_{5}\right)\left[\operatorname{sen}^{2} \phi_{0}\left(v_{, x}\right)+\operatorname{sen} \phi_{0} \cos \phi_{0}\left(w_{, x}\right)\right], \\
\Phi^{x z} & =\frac{1}{2}\left(\alpha_{2}+\alpha_{5}\right)\left[\cos ^{2} \phi_{0}\left(w_{, x}\right)+\operatorname{sen} \phi_{0} \cos \phi_{0}\left(v_{, x}\right)\right], \\
\Phi^{y x} & =\frac{1}{2}\left(\alpha_{3}+\alpha_{6}\right)\left[\operatorname{sen}^{2} \phi_{0}\left(v_{, x}\right)+\operatorname{sen} \phi_{0} \cos \phi_{0}\left(w_{, x}\right)\right], \\
\Phi^{y y} & =0, \\
\Phi^{y z} & =0, \\
\Phi^{z x} & =\frac{1}{2}\left(\alpha_{3}+\alpha_{6}\right)\left[\cos ^{2} \phi_{0}\left(w_{, x}\right)+\operatorname{sen} \phi_{0} \cos \phi_{0}\left(v_{, x}\right)\right], \\
\Phi^{z y} & =0, \\
\Phi^{z z} & =0,
\end{aligned}
$$

onde

$$
\begin{aligned}
v_{, x} & =-\frac{\left(\eta_{3}-\eta_{1}\right)}{2 \eta_{3} \eta_{1}} \operatorname{sen} \phi_{0} \cos \phi_{0} \mathcal{A}(D-2 x), \\
w_{, x} & =-\frac{\left(\eta_{1} \operatorname{sen}^{2} \phi_{0}+\eta_{3} \cos ^{2} \phi_{0}\right)}{2 \eta_{3} \eta_{1}} \mathcal{A}(D-2 x) .
\end{aligned}
$$

Como mencionado na Seção 2.1, a viscosidade $\eta_{3}=\frac{1}{2} \alpha_{4}$ corresponde à viscosidade isotrópica newtoniana, e quando tomamos $\eta_{1}=\eta_{3}$ as soluções analíticas (2.4) e (2.5) reduzem-se ao clássico escoamento de Poiseuille (ver equação (2.9)). As equações (2.4) e (2.5) mostram que se $\phi_{0} \neq 0$ ou $\phi_{0} \neq 90^{\circ}$ então pode-se observar que ocorre um escoamento transversal gerado pela velocidade $v(x)$. Este escoamento transversal é chamado de componente transversal do escoamento ou segunda componente do escoamento (ver [123]). 


\subsubsection{Comparação entre as Soluções Numéricas e Analítica}

Para efeito de verificação do método numérico, será simulado o escoamento de um cristal líquido nemático entre duas placas paralelas sob forte campo magnético. Assim, considere o escoamento tridimensional entre duas placas paralelas separadas por uma distância $D$, largura $L$ e comprimento $C$, como mostrado na Figura 2.4 .

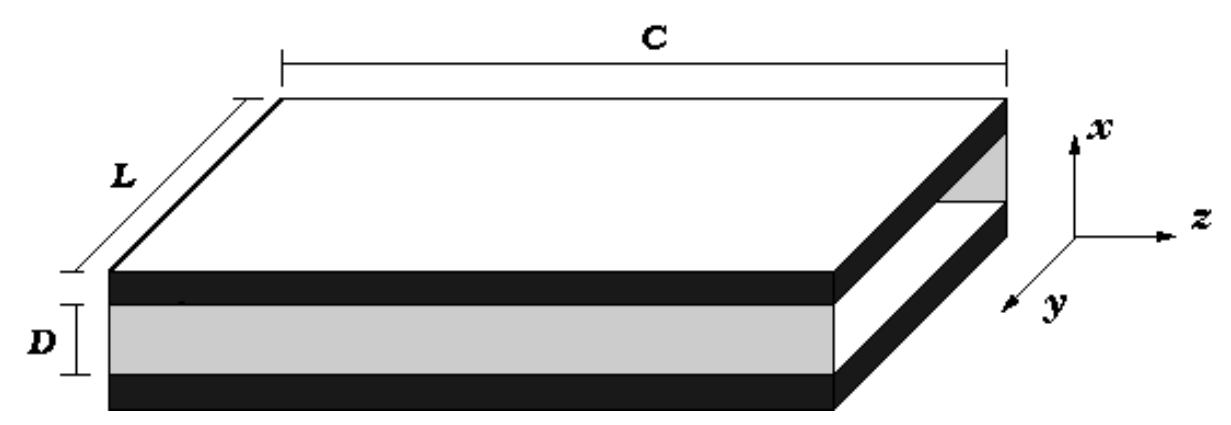

Figura 2.4: Definição do domínio de escoamento para a simulação do escoamento tridimensional entre duas placas paralelas. As paredes cinzas representam as paredes laterais fictícias.

Na entrada das placas, $0 \leq x \leq D, 0 \leq y \leq L, z=0$, as seguintes condições foram impostas:

$$
u=0, \quad v=0 \quad \text { e } \quad w=U
$$

e na saída entre as placas , $0 \leq x \leq D, 0 \leq y \leq L, z=C$, as seguintes condições foram impostas:

$$
u_{, z}=v_{, z}=w_{, z}=0 .
$$

Nas paredes das placas, $x=0,0 \leq y \leq L, 0 \leq z \leq C$ e $x=D, 0 \leq y \leq L, 0 \leq z \leq C$, a condição de não-deslizamento (no-slip condition) foi imposta:

$$
u=v=w=0 .
$$

Nas laterais (fictícias), $y=0,0 \leq x \leq D, 0 \leq z \leq C$ e $y=L, 0 \leq x \leq D, 0 \leq z \leq C$, adotamos as seguintes condições:

$$
u=0, \quad v_{, y}=w_{, y}=0 .
$$

Entre as placas, $0 \leq x \leq D, 0 \leq y \leq L, 0 \leq z \leq C$, a velocidade inicial do fluido foi prescrita como $u=v=0$ e $w=U$.

As simulações foram realizadas utilizando os seguintes parâmetros físicos associados com o cristal líquido $\mathrm{MBBA}$ à $25^{\circ} \mathrm{C}$ :

- viscosidade: $\mu=\frac{1}{2} \alpha_{4}=0.0413$ Pa. s; 
- parâmetros físicos, por exemplo as viscosidades de Leslie, densidade, etc, são dadas na Tabela A.2 no Apêndice A.

Os parâmetros restantes especificando o escoamento são:

- largura das placas: $L=0.5 \mathrm{~m}$;

- comprimento das placas: $C=1.0 \mathrm{~m}$;

- distância entre as placas: $D=0.1 \mathrm{~m}$;

- tempo inicial $=0$ s; tempo final $=200.0 \mathrm{~s}$;

- velocidade no inflow: $U=0.009550 \mathrm{~ms}^{-1}$;

- passo temporal inicial: $\delta t=0.000001 \mathrm{~s}$;

- ângulos do campo magnético: $\phi_{0}=0^{\circ}, 15^{\circ}, 30^{\circ}, 45^{\circ}, 60^{\circ}, 75^{\circ}$.

Com estes dados obtém-se

$$
R e=\frac{\rho U D}{\mu} \approx 25.158
$$

Para analisar a convergência do método numérico desenvolvido, esse problema foi simulado utilizando três malhas:

- Malha 1 (M1): $10 \times 50 \times 100$ células $(\delta x=\delta y=\delta z=0.01) \mathrm{m}$;

- Malha $2($ M2): $20 \times 100 \times 200$ células $(\delta x=\delta y=\delta z=0.005) \mathrm{m}$;

- Malha 3 (M3): $25 \times 125 \times 250$ células $(\delta x=\delta y=\delta z=0.004) \mathrm{m}$.

Os resultados dessas simulações são mostrados e analisados a seguir.

Utilizando estes dados o código Freeflow3D simulou o escoamento tridimensional entre duas placas até $t=200$. Para cada malha seis simulações foram realizadas correspondendo aos seis valores do ângulo do campo magnético $\phi_{0}$ especificado acima. Espera-se que no tempo $t=200$ o estado estacionário tenha sido alcançado e que as soluções numéricas aproximem a solução analítica.

De fato, para demonstrar que a solução estacionária foi encontrada, a velocidade transversal $v$ e as respectivas curvas de nível em diferentes planos ao longo do escoamento nos tempos $t=10$, 50 e 200, para $\phi_{0}=30^{\circ}$, são mostrados nas Figuras 2.5, 2.6 e 2.7. Espera-se que a solução numérica varie em função de $x$ para valores de $z$ próximo ao outflow $(z=C)$.

Para verificar este fato, as Figuras 2.5, 2.6 e 2.7 exibem as curvas de nível da velocidade $v$ no plano $x y$ para três valores de $z$ juntamente com as curvas de nível da velocidade $v$ nos planos $y z$ e $x z$. Pode-se notar que em $t=10$ s (Figura 2.5) o escoamento ainda não está totalmente desenvolvido e a velocidade $v$ é praticamente nula em todo o domínio. Entretanto, para $t=50 \mathrm{~s}$ 
(Figura 2.6) pode ser observado que a velocidade está em desenvolvimento e tem valores não nulos entre as placas. Finalmente, para $t=200$ s (Figura 2.7) pode-se observar que a velocidade $v$ próximo da saídas das placas varia somente como uma função de $x$.

Por outro lado, as Figuras 2.8, 2.9 e 2.10 mostram a velocidade $w$ e as respectivas curvas de nível nos tempos $t=10 \mathrm{~s}, 50 \mathrm{~s}$ e $200 \mathrm{~s}$, nos mesmos planos onde foi mostrada a velocidade $v$. Novamente, pode-se observar nas Figuras 2.8, 2.9 e 2.10 que para valores próximos a saída das placas $(z=C)$, a velocidade $w$ varia somente como uma função de $x$. De maneira análoga, a Figura 2.11 exibem as curvas de nível da pressão (isobars) $p$ em $t=200$ s e pode ser visto que a pressão varia somente como uma função de $z$.

Portanto, os resultados das Figuras $(2.5,2.6$ e 2.7) e $(2.8,2.9$ e 2.10) mostram que para $t=200$ s o estado estacionário foi alcançado e que as soluções numéricas,para valores próximo $z=C$, variam somente como uma função de $x$. Os resultados numéricos obtidos, para outros valores do ângulo $\phi_{0}$, mostraram um comportamento semelhante.

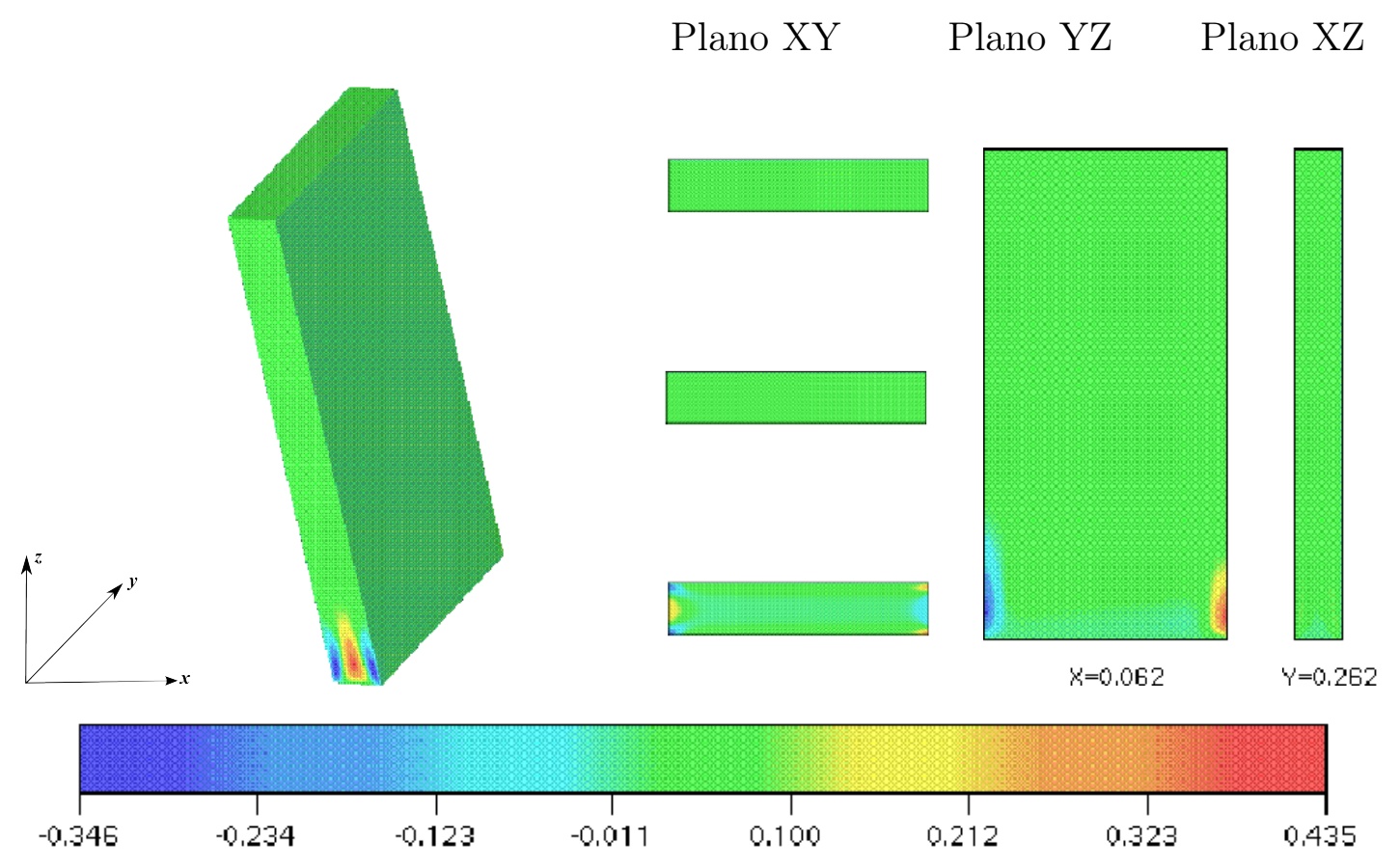

Figura 2.5: Isolinhas da velocidade $v$ em $t=10$ s: curvas de nível obtidas em vários planos ao longo das placas. - $\phi_{0}=30^{\circ}$. Entrada de fluido pela parte inferior. 


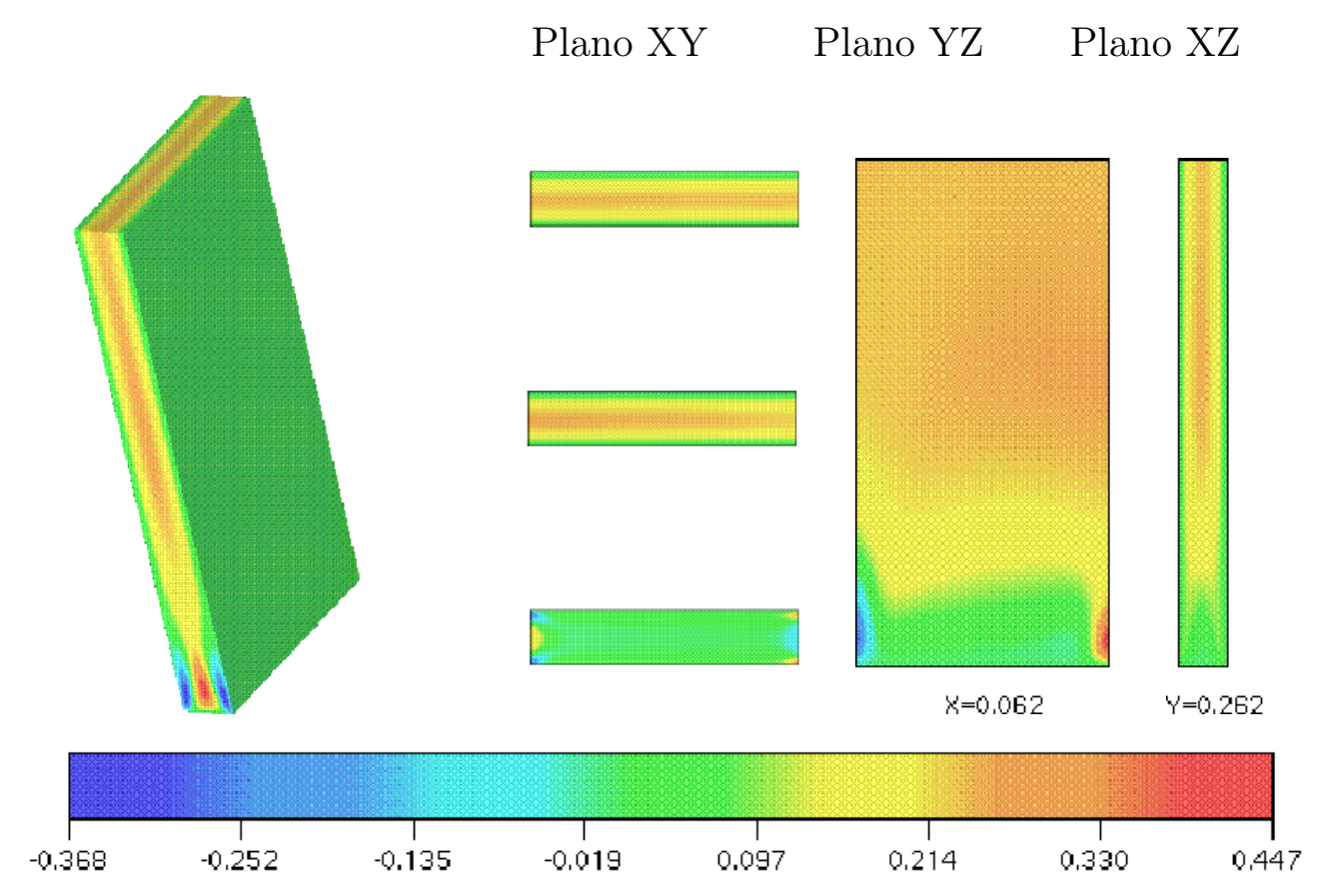

Figura 2.6: Isolinhas da velocidade $v$ em $t=50 \mathrm{~s}$ : curvas de nível obtidas em vários planos ao longo das placas. $-\phi_{0}=30^{\circ}$. Entrada de fluido pela parte inferior.

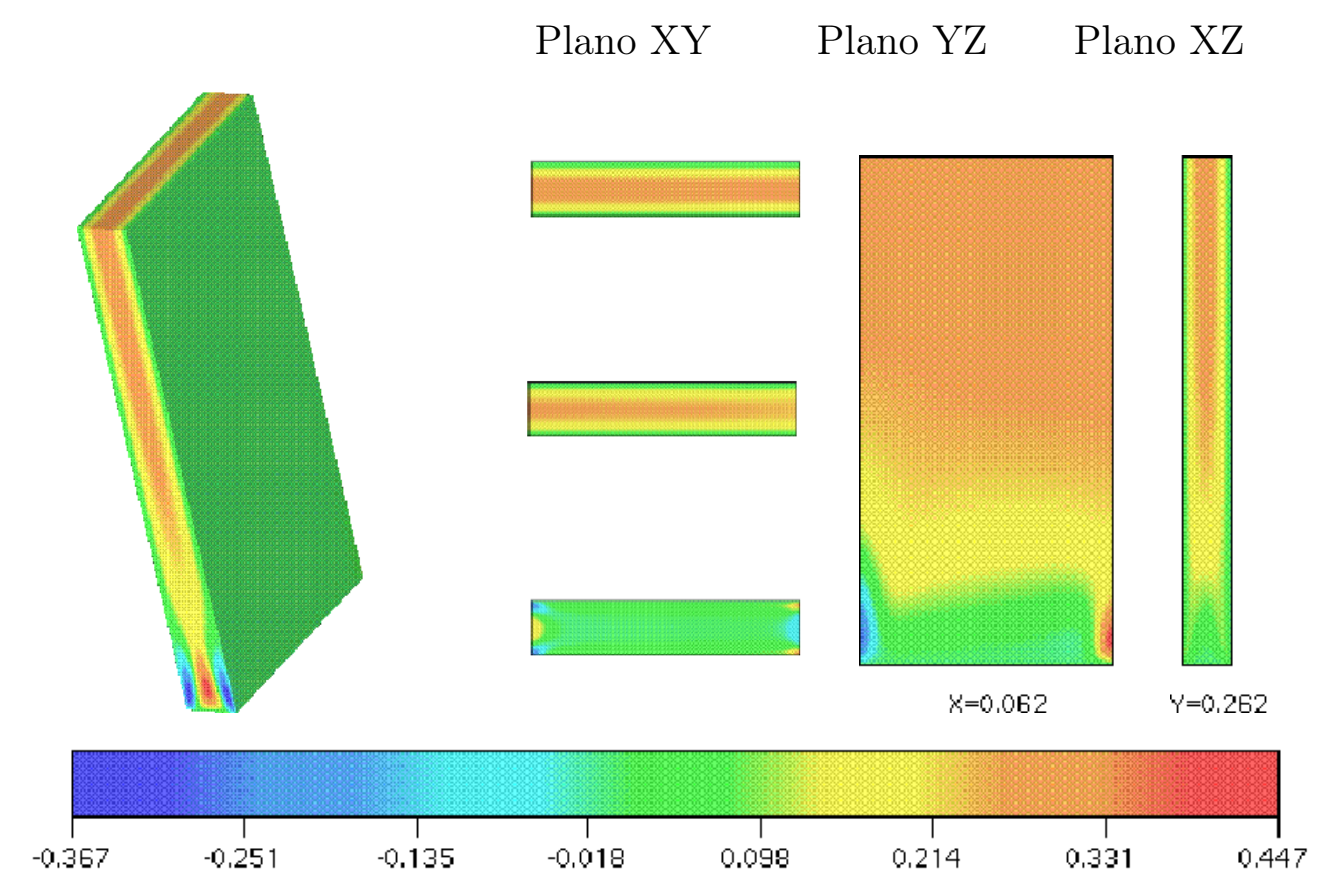

Figura 2.7: Isolinhas da velocidade $v$ em $t=200$ s: curvas de nível obtidas em vários planos ao longo das placas. - $\phi_{0}=30^{\circ}$. Entrada de fluido pela parte inferior. 


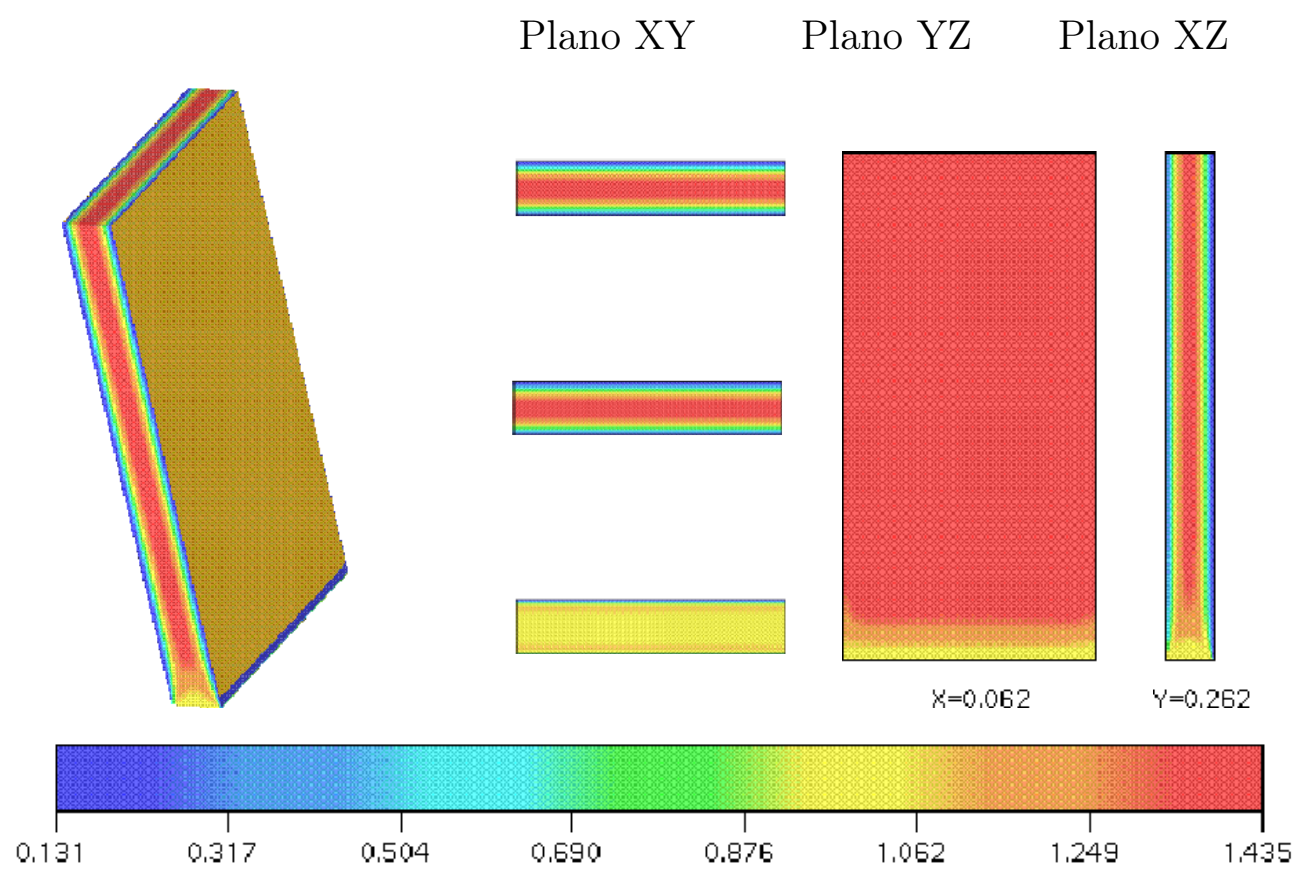

Figura 2.8: Isolinhas da velocidade $w$ em $t=10 \mathrm{~s}$ : curvas de nível obtidas em vários planos ao longo das placas. $-\phi_{0}=30^{\circ}$. Entrada de fluido pela parte inferior.

Plano XY Plano YZ Plano XZ
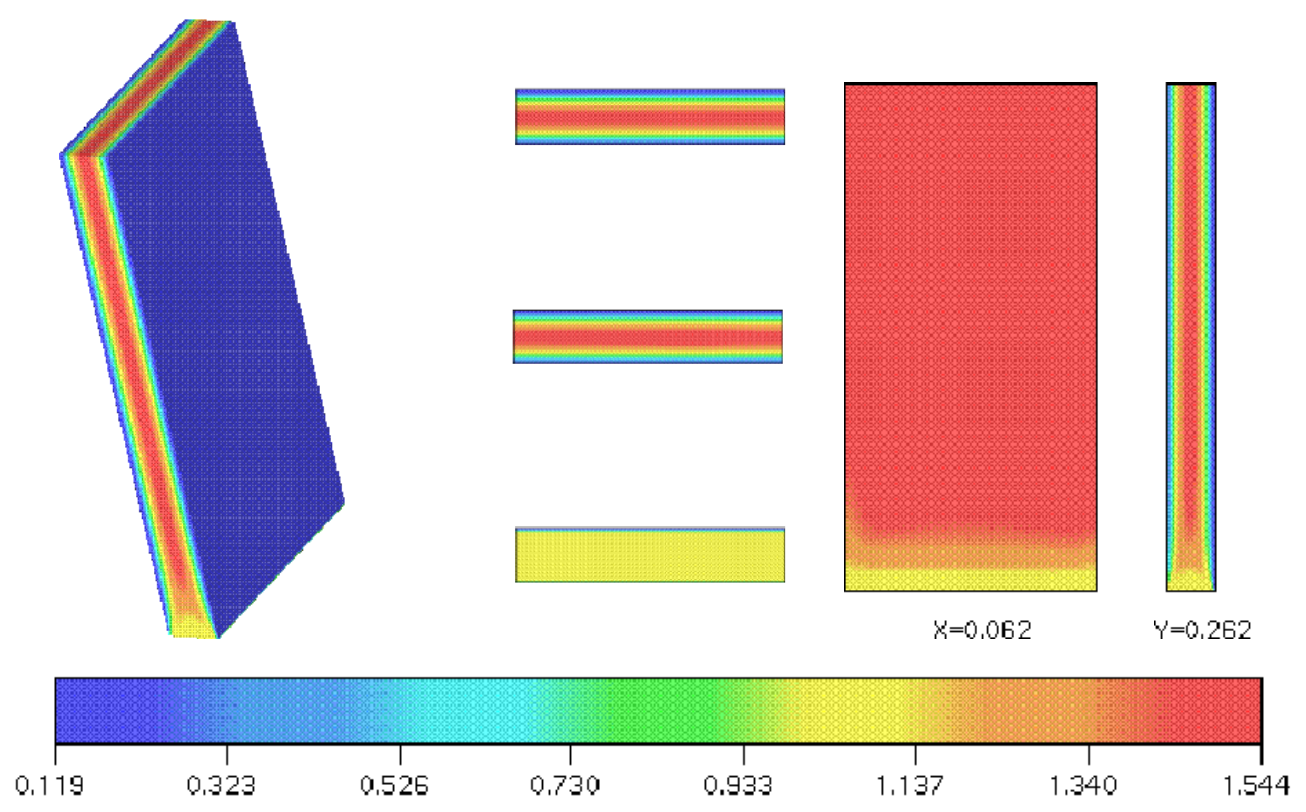

Figura 2.9: Isolinhas da velocidade $w$ em $t=50$ s: curvas de nível obtidas em vários planos ao longo das placas. $-\phi_{0}=30^{\circ}$. Entrada de fluido pela parte inferior. 


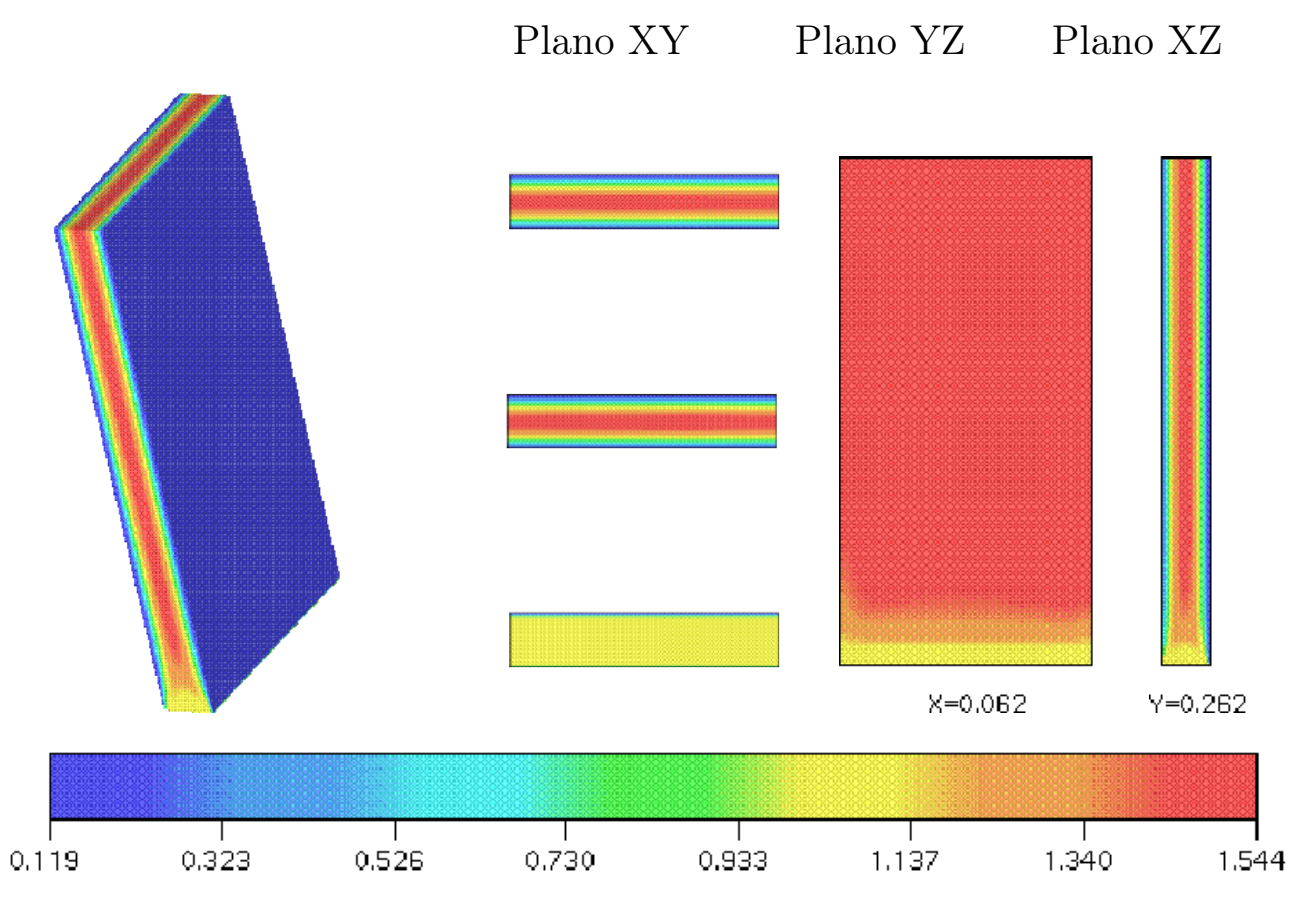

Figura 2.10: Isolinhas da velocidade $w$ em $t=200$ s: curvas de nível obtidas em vários planos ao longo das placas. - $\phi_{0}=30^{\circ}$. Entrada de fluido pela parte inferior.

$$
\text { Plano XY Plano YZ Plano XZ }
$$
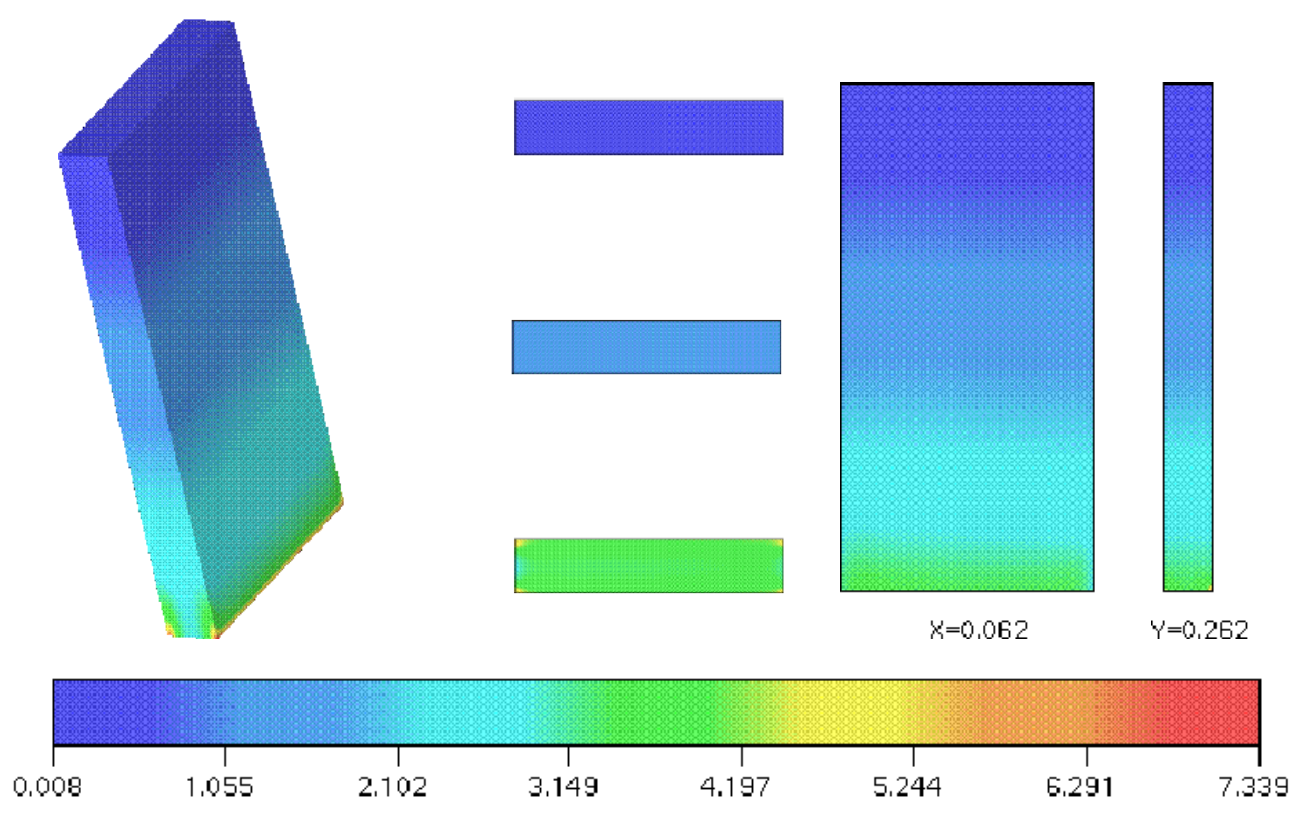

Figura 2.11: Isolinhas da pressão $p$ em $t=200$ s: curvas de nível obtidas em vários planos ao longo das placas. Entrada de fluido pela parte inferior. 


\subsubsection{Avaliação da Solução Analítica}

Para avaliar a solução analítica dada pelas equações (2.4) e (2.5), o gradiente de pressão precisa ser conhecido. Um valor apropriado de $\mathcal{A}$ pode ser determinado calculando a vazão na saída entre as placas usando $w(x)$ dado por (2.5) e iguala-se com a vazão obtida na entrada entre as placas. Por exemplo, para $\phi_{0}=30^{\circ}$, este procedimento encontra que $\mathcal{A}=-0.3066539$. Para outros valores do ângulo $\phi_{0}$, o gradiente de pressão foi determinado de maneira semelhante.

A Figura 2.12 compara a solução analítica para as componentes da velocidade $v$ e $w$ com suas respectivas soluções numéricas obtidas nas malhas M1, M2 e M3 para $\phi_{0}=0^{\circ}, 15^{\circ}, 30^{\circ}$, $\phi_{0}=45^{\circ}, 60^{\circ}$ e $\phi_{0}=75^{\circ}$. Pode-se observar que as soluções numéricas concordam com suas respectivas soluções analíticas. Além disso, observa-se que as soluções numéricas convergem para a solução analítica quando a malha é refinada.

Para mostrar quantitativamente que o método numérico é convergente, o erro relativo da solução numérica $\Pi_{\text {Numerica }}\left(\operatorname{ver}(2.96)\right.$ ), para cada valor do ângulo $\phi_{0}$, foi calculado nas três malhas pela equação

$$
E(\Pi)=\sqrt{\frac{\sum_{i j}\left(\Pi_{\text {Exata }}-\Pi_{\text {Numerica }}\right)^{2}}{\sum_{i j}\left(\Pi_{\text {Exata }}\right)^{2}}} .
$$

Os erros foram calculados no plano $y z$ para $y=0.25 \mathrm{~m}$ e $z=(1.0-3 \delta z) \mathrm{m}$. Os valores dos erros relativos obtidos nas malhas M1, M2 e M3 são mostrados nas Tabelas 2.1 e 2.2. Pode ser notado nas Tabelas 2.1 e 2.2 que os erros relativos decrescem à medida que a malha é refinada, indicando que o método numérico é de fato convergente. Além disso, utilizando os resultados mostrados nas Tabelas 2.1 e 2.2, ângulo $\phi_{0}=30^{\circ}$, a ordem de convergência aproximada do método foi calculada pela fórmula

$$
N_{i}(*)=\frac{\log \left[\frac{E(*)_{M_{i+1}}}{E(*)_{M_{i}}}\right]}{\log \left[\frac{\delta x_{M_{i+1}}}{\delta x_{M_{i}}}\right]}, i=1,2 .
$$

Para a velocidade $v$ obteve-se $N_{1}(v)=0.65$ e $N_{2}(v)=1.32$ enquanto para a velocidade $w$ foi obtido $N_{1}(w)=1.56$ e $N_{2}(w)=0.94$. Estes resultados confirmam que o método numérico desenvolvido nesse trabalho é convergente.

Um outro resultado importante obtido nesse trabalho é a determinação do ângulo de deflexão $\varphi$ dado pela equação (2.83) (ver Stewart [123]). Pieranski e Guyon [105] determinaram experimentalmente este ângulo para cristais líquidos nemáticos MBBA e foi constatado que o ângulo de deflexão $\varphi$ determinado pela solução analítica concorda com o valor obtido experimentalmente. A Figura 2.13 compara a solução analítica (2.83) com a solução numérica obtida nas três malhas M1, M2 e M3. Pode-se observar da Figura 2.13 que a solução numérica está em boa concordância com a solução analítica. Finalmente, as Figuras 2.14, 2.15 e 2.16 exibem 


\begin{tabular}{|c|c|c|c|c|c|}
\hline \multirow[b]{2}{*}{$\phi_{0}$} & \multirow{2}{*}{$\begin{array}{c}\text { Erro relativo } \\
\text { M1 }\end{array}$} & \multirow{2}{*}{$\begin{array}{c}\text { Erro relativo } \\
\text { M2 }\end{array}$} & \multirow{2}{*}{$\begin{array}{c}\text { Erro relativo } \\
\text { M3 }\end{array}$} & \multicolumn{2}{|c|}{ Ordem de convergência } \\
\hline & & & & $N_{1}$ & $N_{2}$ \\
\hline 0 & 0.000 & 0.000 & 0.000 & 0 & 0 \\
\hline 15 & $4.3046 \times 10^{-2}$ & $3.2204 \times 10^{-2}$ & $2.1062 \times 10^{-2}$ & 0.42 & 1.90 \\
\hline 30 & $7.6209 \times 10^{-2}$ & $4.8532 \times 10^{-2}$ & $3.6122 \times 10^{-2}$ & 0.65 & 1.32 \\
\hline 45 & $8.1976 \times 10^{-2}$ & $6.8247 \times 10^{-2}$ & $5.3888 \times 10^{-2}$ & 0.26 & 1.06 \\
\hline 60 & $1.1402 \times 10^{-1}$ & $9.4843 \times 10^{-2}$ & $7.6157 \times 10^{-2}$ & 0.27 & 0.98 \\
\hline 75 & $1.4553 \times 10^{-1}$ & $1.1803 \times 10^{-1}$ & $9.8473 \times 10^{-2}$ & 0.30 & 0.81 \\
\hline
\end{tabular}

Tabela 2.1: Erro relativo na velocidade $v$ nas malhas M1, M2, M3 e ordem de convergência.

\begin{tabular}{|c||c|c|c||c|c|}
\hline \multicolumn{1}{|c||}{} & Erro relativo & Erro relativo & Erro relativo & \multicolumn{2}{c|}{ Ordem de Convergência } \\
\cline { 5 - 6 }$\phi_{0}$ & M1 & M2 & M3 & $N_{1}$ & $N_{2}$ \\
\hline \hline 0 & $8.7279 \times 10^{-3}$ & $3.3542 \times 10^{-3}$ & $3.0390 \times 10^{-3}$ & 1.38 & 0.44 \\
\hline 15 & $8.2253 \times 10^{-3}$ & $3.1962 \times 10^{-3}$ & $2.9023 \times 10^{-3}$ & 1.36 & 0.43 \\
\hline 30 & $1.5588 \times 10^{-2}$ & $5.2912 \times 10^{-3}$ & $4.2912 \times 10^{-3}$ & 1.56 & 0.94 \\
\hline 45 & $2.0312 \times 10^{-2}$ & $5.6595 \times 10^{-3}$ & $5.0131 \times 10^{-3}$ & 1.84 & 0.54 \\
\hline 60 & $1.7834 \times 10^{-2}$ & $6.4032 \times 10^{-3}$ & $5.5072 \times 10^{-3}$ & 1.48 & 0.68 \\
\hline 75 & $1.8331 \times 10^{-2}$ & $5.4201 \times 10^{-3}$ & $5.0104 \times 10^{-3}$ & 1.76 & 0.27 \\
\hline
\end{tabular}

Tabela 2.2: Erro relativo na velocidade $w$ nas malhas M1, M2, M3 e ordem de convergência.

a comparação da solução analítica para as componentes do tensor das tensões não-newtoniano (equações (2.85)-(2.87) e (2.90) com suas respectivas soluções numéricas para $\phi_{0}=0^{\circ}, 30^{\circ} \mathrm{e}$ $60^{\circ}$; novamente, boa concordância entre as duas soluções foi obtida. Observa-se também que a solução numérica converge para sua respectiva solução analítica quando a malha é refinada. Para outros valores do ângulo $\phi_{0}$ resultados similares foram obtidos.

Estes resultados fornecem uma validação do método numérico (e respectivo software associado) para resolver escoamentos tridimensionais de cristais líquidos nemáticos sujeitos à um forte campo magnético. 

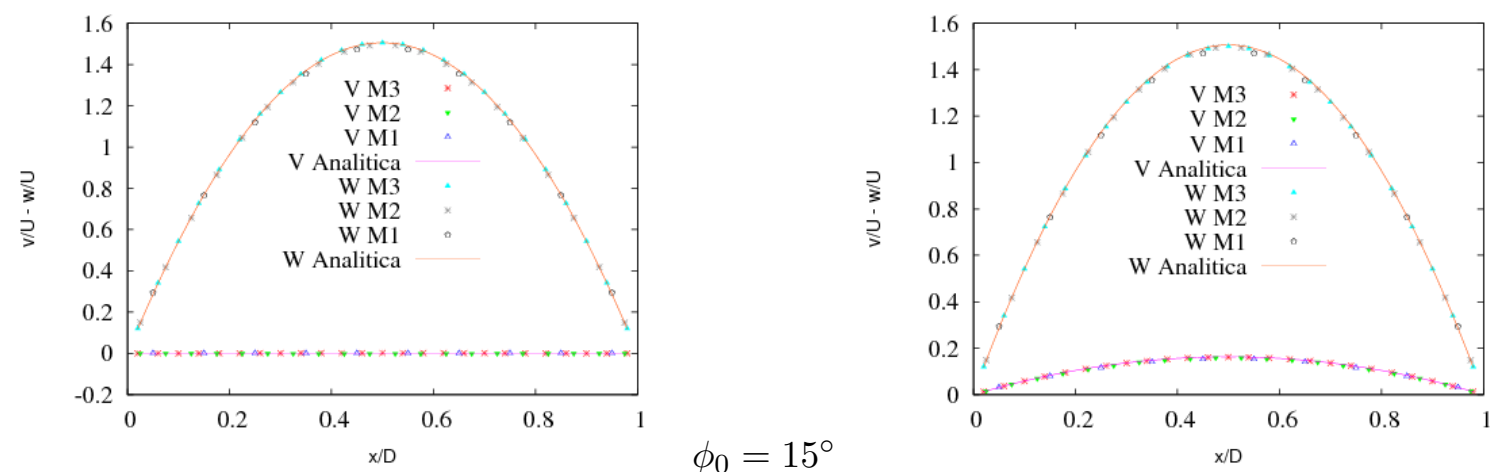

$\phi_{0}=0^{\circ}$

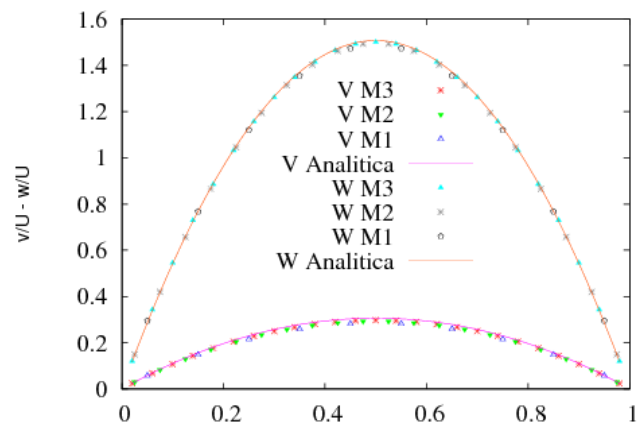

$\phi_{0}=15^{\circ}$

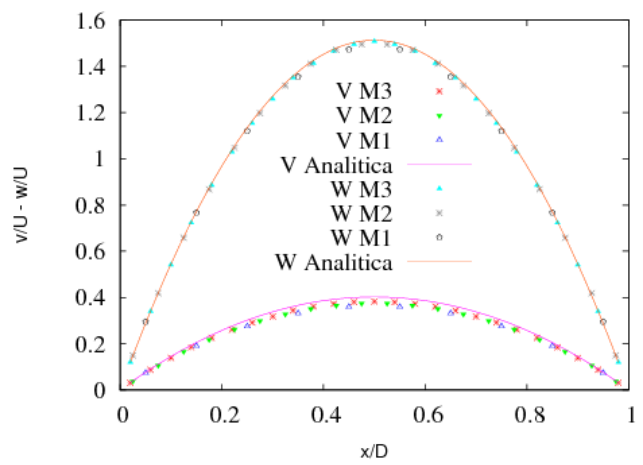

$\phi_{0}=30^{\circ}$

$x / D$

$\phi_{0}=45^{\circ}$

$\mathrm{x} / \mathrm{D}$

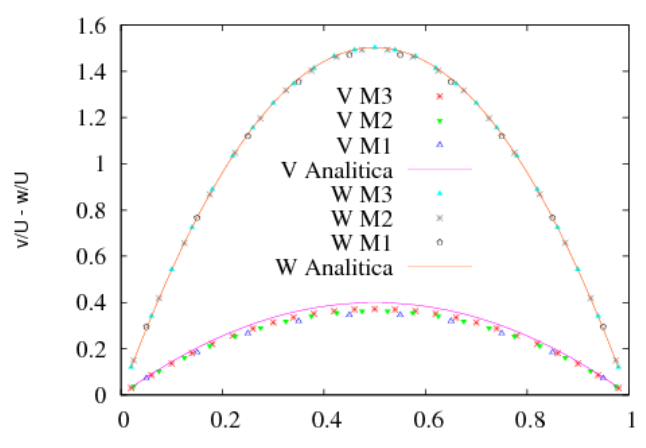

$\phi_{0}=60^{\circ}$

$x / D$

$\phi_{0}=75^{\circ}$

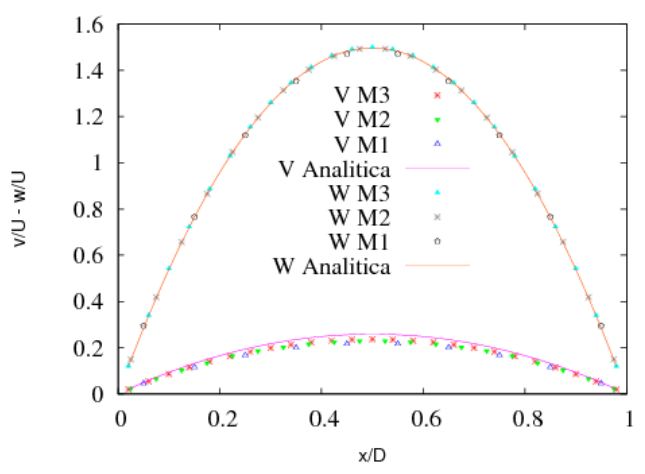

Figura 2.12: Soluções numéricas e analítica das velocidades $v$ e $w$ diversos valores do ângulos $\phi_{0}$. 


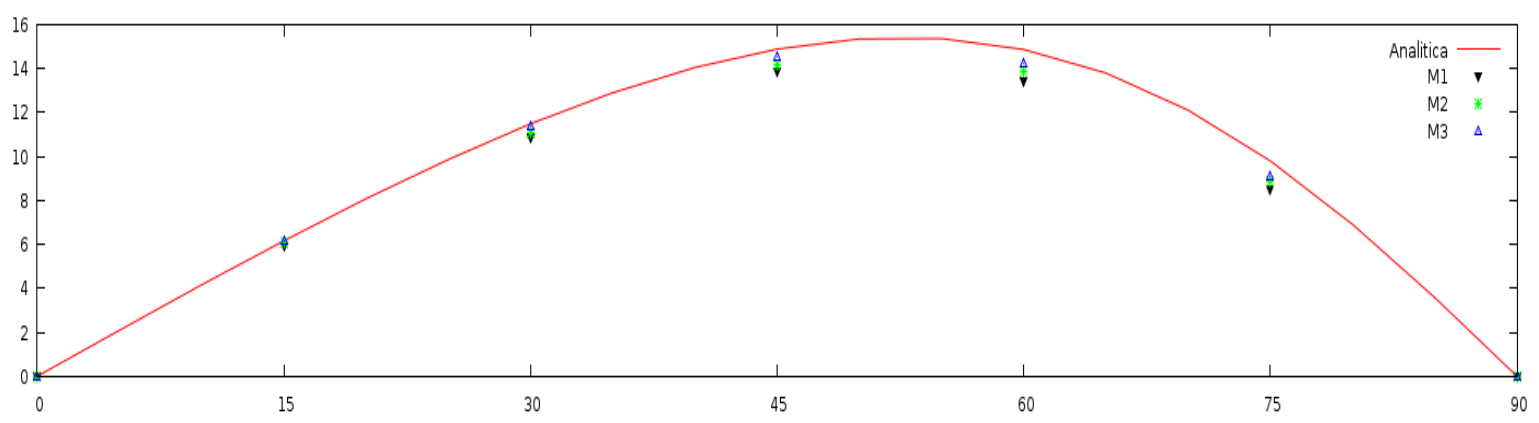

Figura 2.13: Variação do ângulo de deflexão $\varphi$ em função do ângulo $\phi$. Comparação entre resultados numéricos e valores analíticos dado pela equação (2.83).
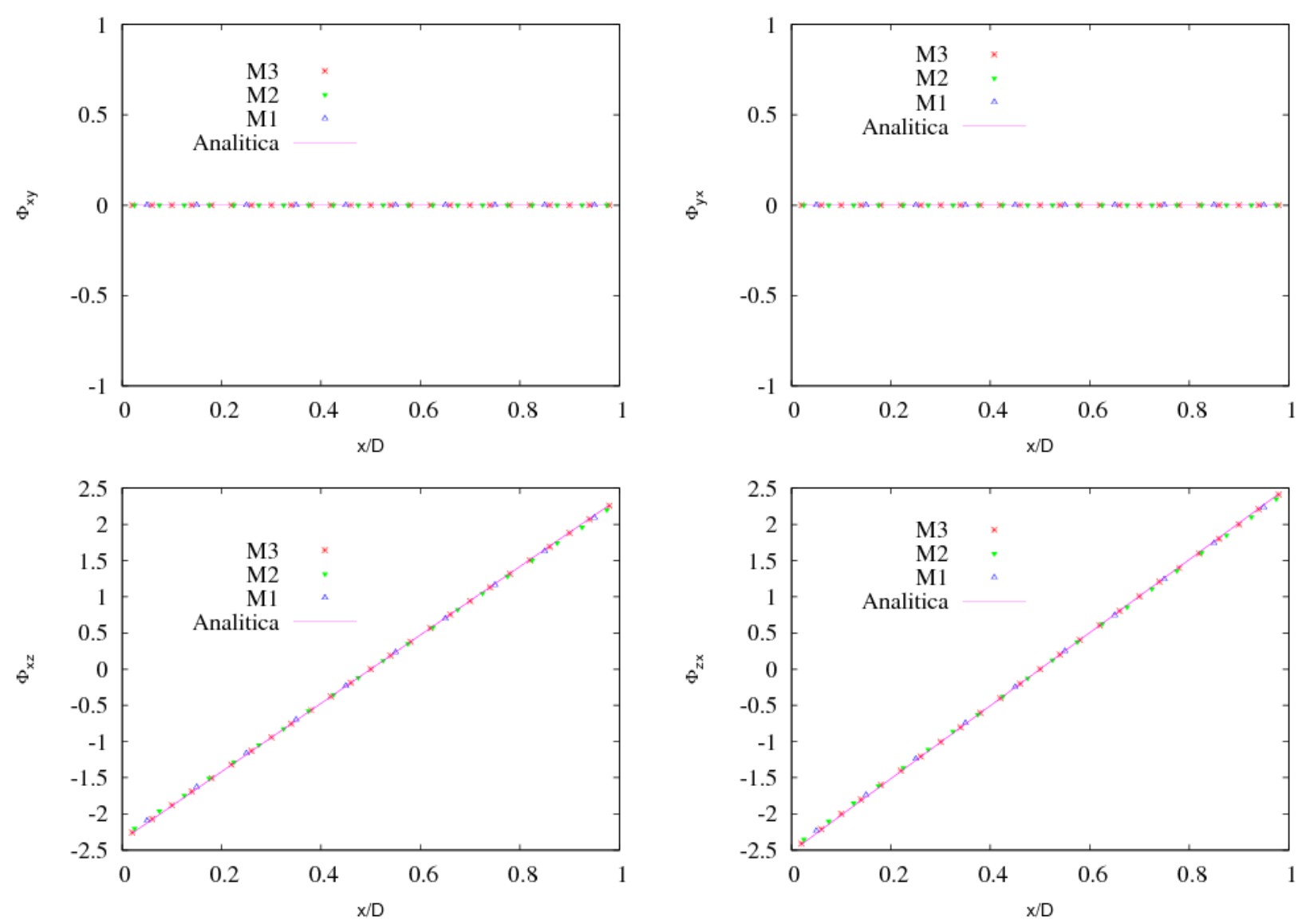

Figura 2.14: Comparação entre os resultados numéricos e analíticos das componentes do tensor das tensões não newtoniano $\Phi^{x y}, \Phi^{y x}, \Phi^{x z}$ e $\Phi^{z x}$ : Ângulo $\phi_{0}=0^{\circ}$. 

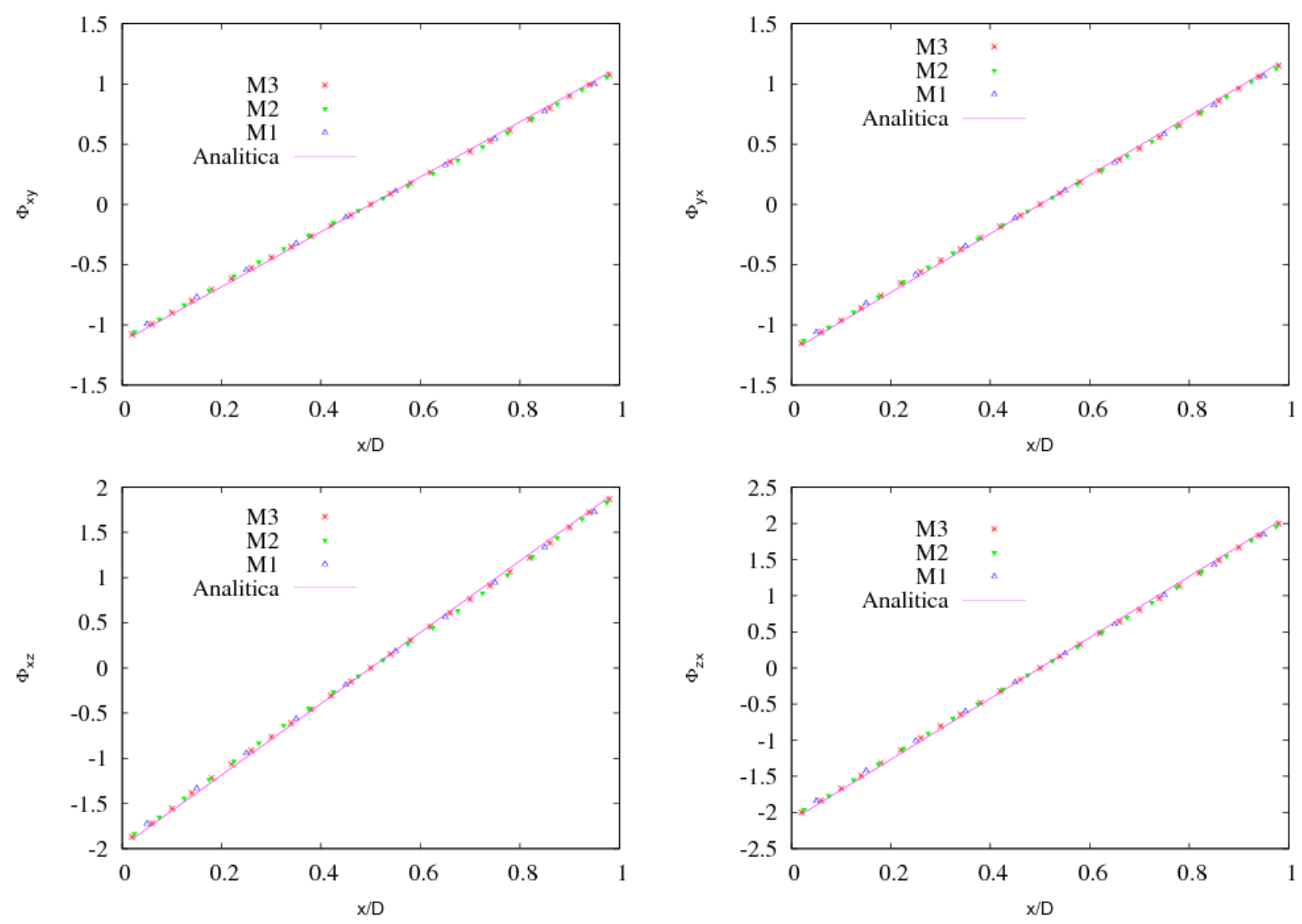

Figura 2.15: Comparação entre os resultados numéricos e analíticos das componentes do tensor das tensões não newtoniano $\Phi^{x y}, \Phi^{y x}, \Phi^{x z}$ e $\Phi^{z x}$ : Ângulo $\phi_{0}=30^{\circ}$. 

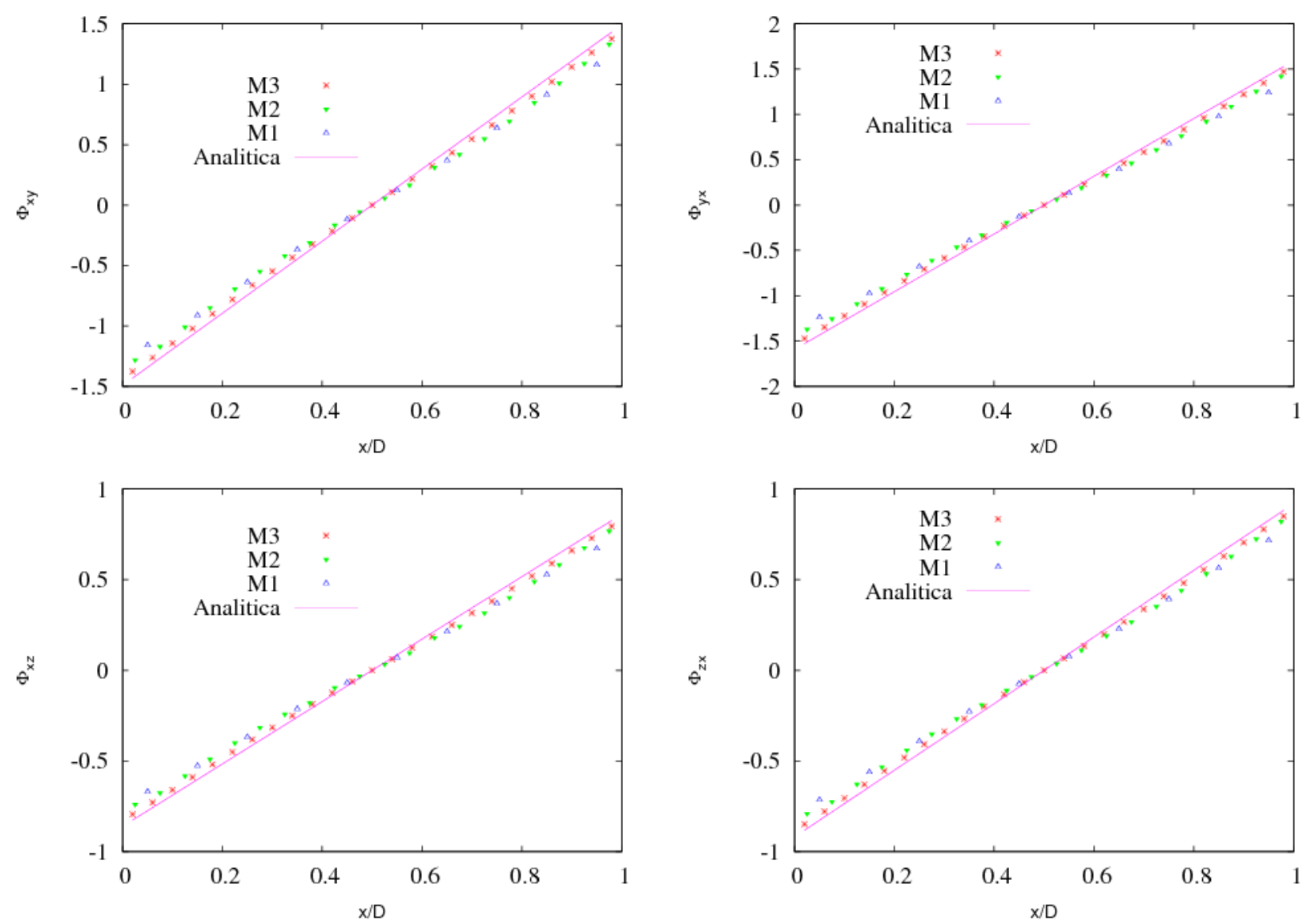

Figura 2.16: Comparação entre os resultados numéricos e analíticos das componentes do tensor das tensões não newtoniano $\Phi^{x y}, \Phi^{y x}, \Phi^{x z}$ e $\Phi^{z x}$ : Ângulo $\phi_{0}=60^{\circ}$. 


\subsubsection{Simulação Numérica do Escoamento em um Canal Tridimensi- onal}

Os dados de entrada empregados para simular o escoamento entre duas placas paralelas na Seção 2.8.2 foram utilizados para simular o escoamento de um cristal líquido nemático no interior de um canal tridimensional formado por quatro paredes sólidas. O domínio do escoamento foi o mesmo empregado no escoamento entre placas paralelas exceto que no lugar de paredes com lados fictícios, definiu-se duas paredes sólidas com a condição de não-deslizamento imposta sobre elas, ou seja, em $y=0,0 \leq x \leq D, 0 \leq z \leq C$ e $y=L, 0 \leq x \leq D, 0 \leq z \leq C$ foi imposta

$$
u=v=w=0 .
$$

As simulações foram realizadas utilizando os parâmetros físicos do cristal líquido nemático MBBA à $25^{\circ} \mathrm{C}$ e a malha M3 $(25 \times 25 \times 250$ células $)$. Todos os outros parâmetros utilizados no escoamento entre as placas paralelas foram mantidos; por exemplo $L, C, D, U, \mu(R e=25.158)$ e considerou-se o caso $\phi_{0}=30^{\circ}$. A simulação iniciou-se no tempo $t=0$ s e terminou em $t=200$ s. Os resultados numéricos obtidos nesta simulação em diferentes tempos são exibidos nas Figuras 2.17-2.21 para $t=200 \mathrm{~s}$. Das Figuras 2.18, 2.20 e 2.21 pode-se observar que no tempo $t=200 \mathrm{~s}$ o escoamento próximo a saída do canal está completamente desenvolvido.

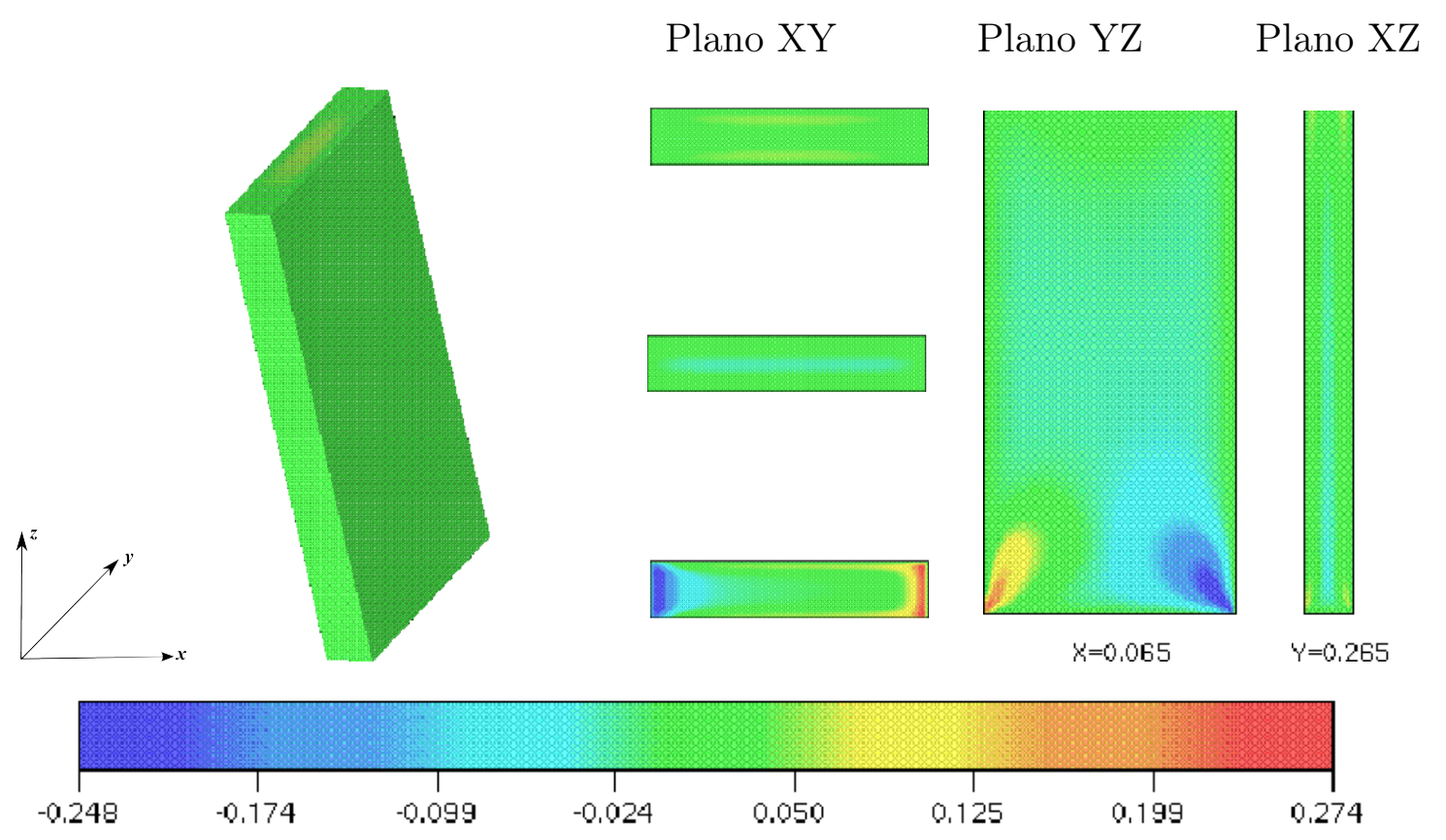

Figura 2.17: Isolinhas da velocidade $v$ em $t=10$ s: curvas de nível obtidas em vários planos ao longo do canal tridimensional. - $\phi_{0}=30^{\circ}$. Entrada de fluido pela parte inferior. 


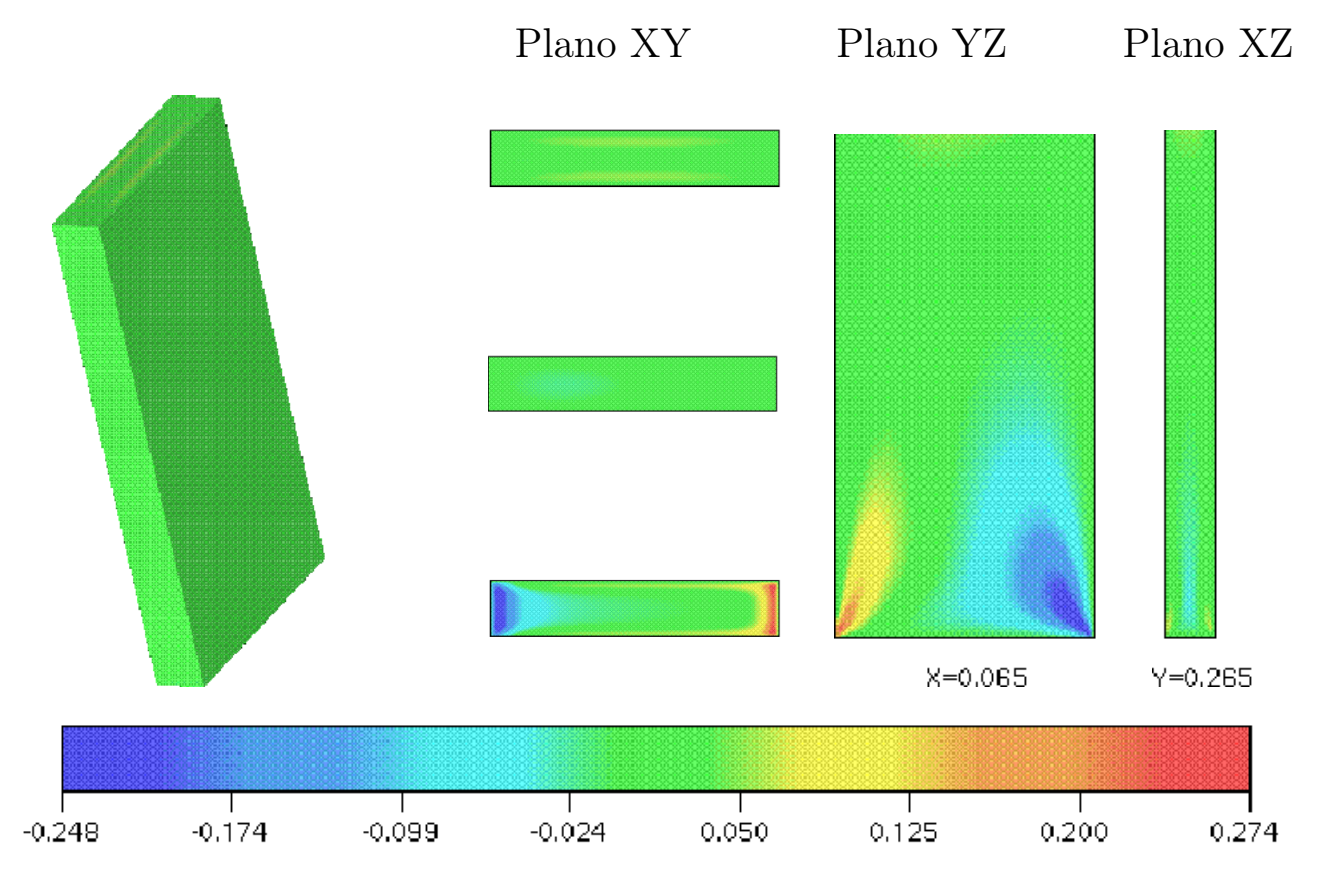

Figura 2.18: Isolinhas da velocidade $v$ em $t=200 \mathrm{~s}$ : curvas de nível obtidas em vários planos ao longo do canal tridimensional. $-\phi_{0}=30^{\circ}$. Entrada de fluido pela parte inferior. 


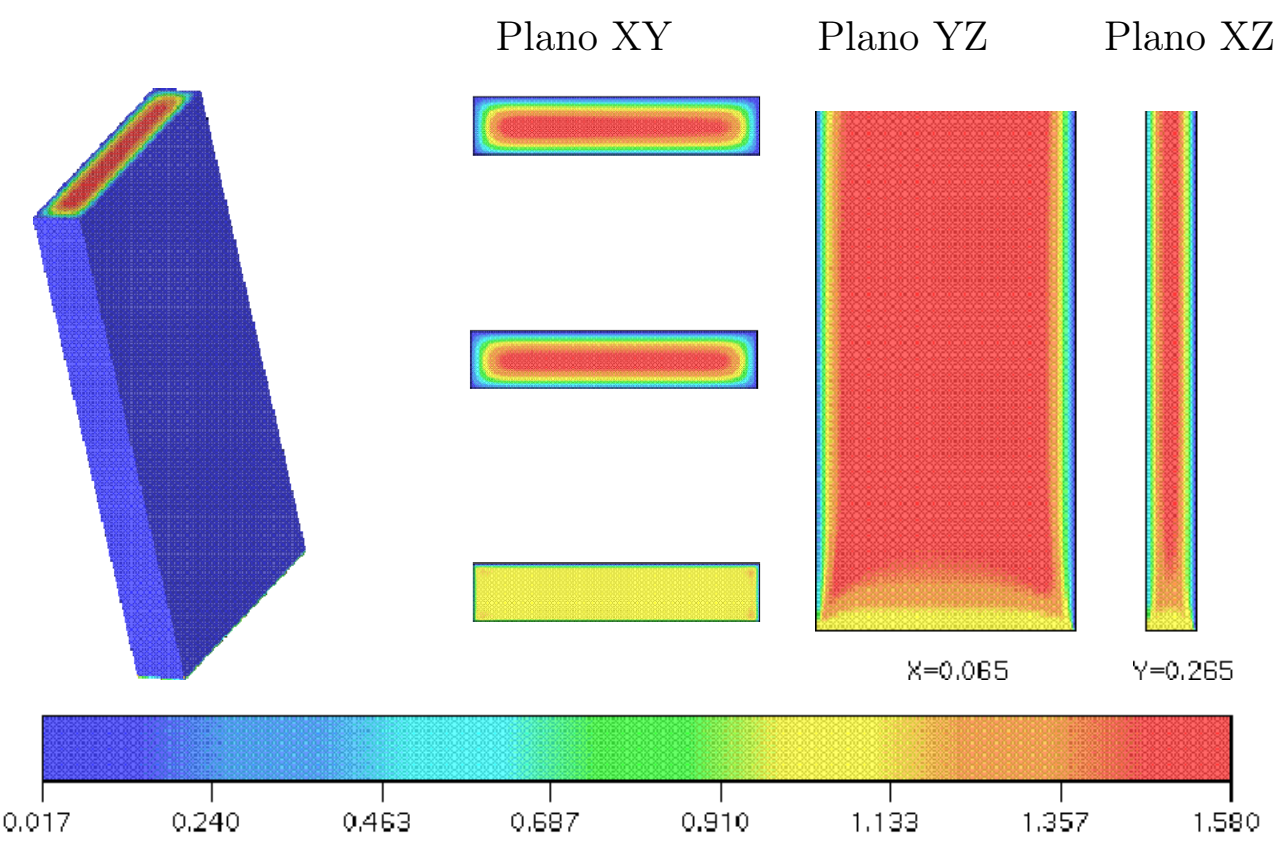

Figura 2.19: Isolinhas da velocidade $w$ em $t=10 \mathrm{~s}$ : curvas de nível obtidas em vários planos ao longo do canal tridimensional.- $\phi_{0}=30^{\circ}$. Entrada de fluido pela parte inferior.

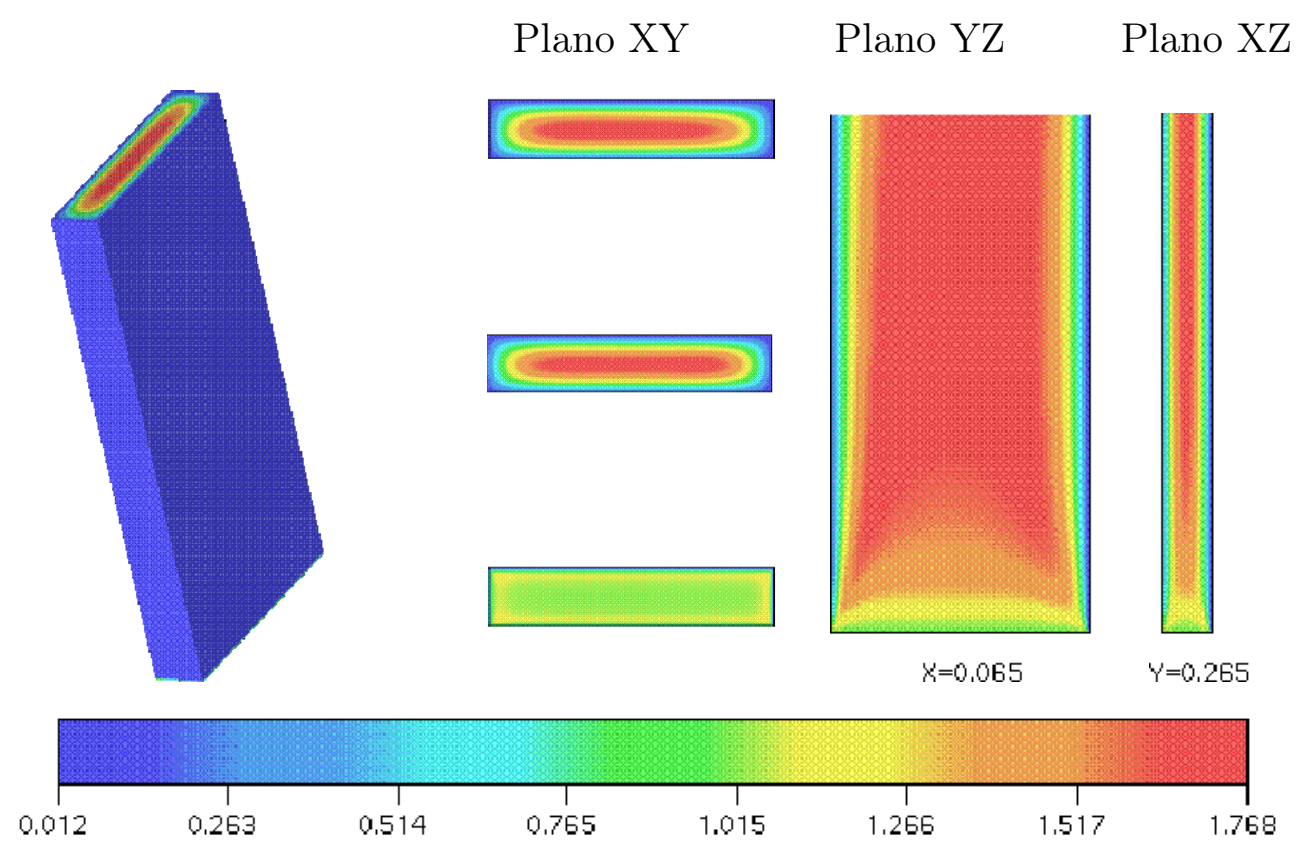

Figura 2.20: Isolinhas da velocidade $w$ em $t=200 \mathrm{~s}$ : curvas de nível obtidas em vários planos ao longo do canal tridimensional. - $\phi_{0}=30^{\circ}$. Entrada de fluido pela parte inferior. 


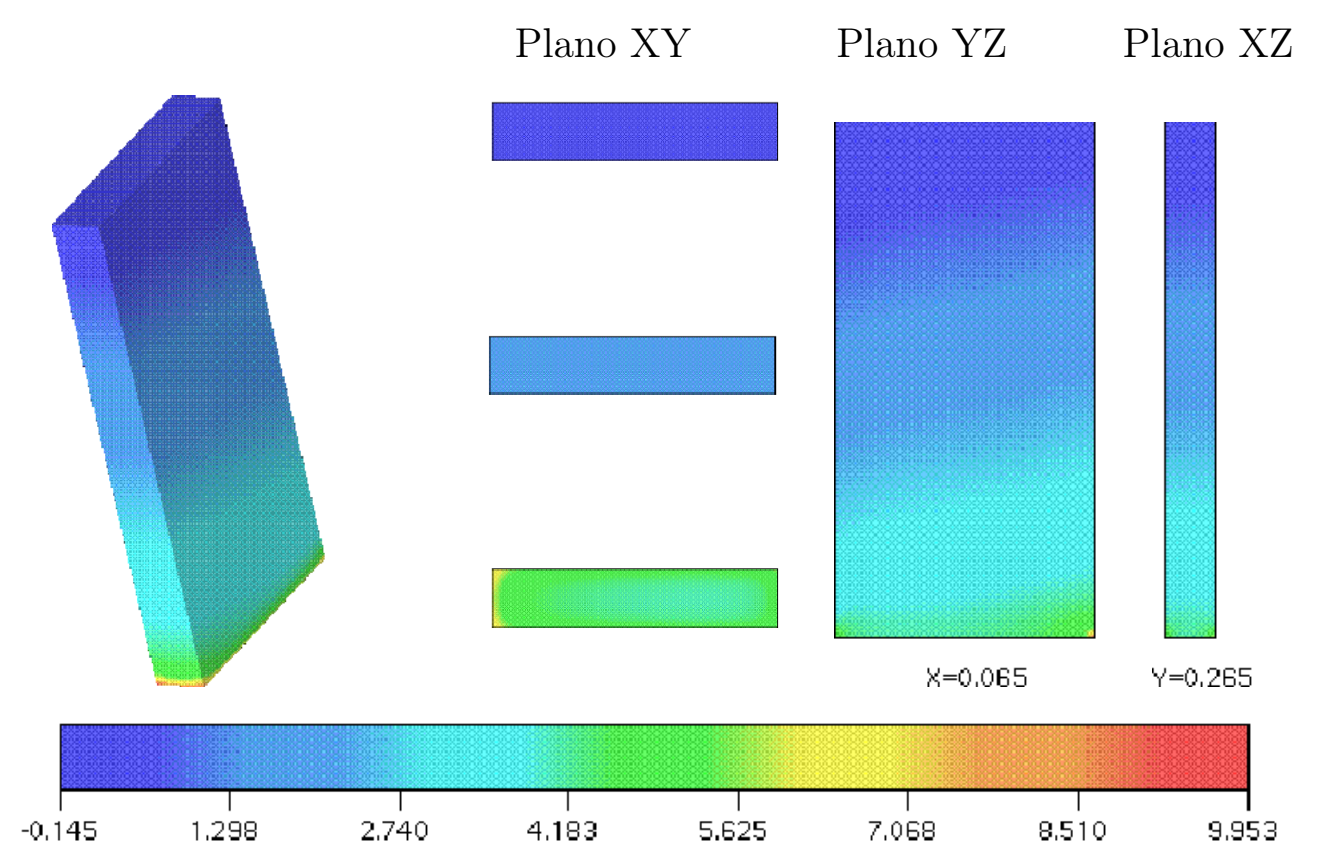

Figura 2.21: Isolinhas da pressão $p$ em $t=200$ s: curvas de nível obtidas em vários planos ao longo do canal tridimensional. - $\phi_{0}=30^{\circ}$. Entrada de fluido pela parte inferior. 


\section{Método Numérico para Escoamentos de Cristais}

\section{Líquidos Nemáticos em Duas Dimensões}

Nesse Capítulo é apresentado um método numérico de diferenças finitas para simular escoamentos bidimensionais de cristais líquidos nemáticos, sob o efeito de um campo magnético, governados pelas equações dinâmicas de Ericksen-Leslie [123]. As equações básicas são apresentadas e são resolvidas por um método numérico baseado na metodologia GENSMAC [134]. As equações que descrevem a técnica numérica desenvolvida nesse Capítulo foram incorporadas ao sistema de simulação Freeflow2D [42]. Empregando as hipóteses de escoamento desenvolvido e pequeno ângulo de orientação do diretor (ver Carou et al. [19, 20, 21]), uma solução analítica para o escoamento em um canal bidimensional foi encontrada. O método numérico foi então validado utilizando essa solução analítica e empregando refinamento de malha, resultados de convergência são apresentados.

\subsection{Equações Dinâmicas de Ericksen-Leslie em Duas Di- mensões}

Considere o escoamento bidimensional de um cristal líquido nemático. Um campo magnético $\mathbf{H}$ é aplicado ao escoamento e assume-se que o ângulo $\theta$ de orientação do diretor é nulo. Desse modo, o diretor $\mathbf{n}$ e a velocidade $\mathbf{v}$ podem ser escritos nas formas gerais

$$
\begin{aligned}
& \mathbf{n}=(\cos \phi, \operatorname{sen} \phi, 0), \\
& \mathbf{v}=(u(x, y, t), v(x, y, t), 0),
\end{aligned} \quad \phi=\phi(x, y, t),
$$

onde $\phi$ é o ângulo de orientação do diretor. 
O potencial do campo magnético (igual ao negativo da energia magnética) é dado por

$$
\Psi=\frac{1}{2} \mu_{0} \Delta \chi\left(n_{i} \cdot H_{i}\right)^{2}, \quad \mathbf{H}=H\left(\cos \phi_{0}, \operatorname{sen} \phi_{0}, 0\right), \quad \phi_{0}=\text { constante }, \quad|\mathbf{H}|=H<\infty,
$$

onde $\Delta \chi>0$ é a anisotropia magnética e $H$ é a magnitude do campo magnético. A força externa generalizada $G_{i}$ é dada pela equação

$$
G_{i}=\frac{\partial \Psi}{\partial n_{i}}=\mu_{0} \Delta \chi\left(n_{i} \cdot \mathbf{H}\right) H_{i}
$$

onde $\mu_{0}>0$ é a permeabilidade do espaço livre.

As equações relevantes são obtidas da teoria dinâmica dos cristais líquidos pelas equações (1.2) à (1.24). Iniciamos pela investigação da equação de conservação de massa, equações de conservação de quantidade de movimento e equação de conservação de momento angular. A adimensionalização destas equações são apresentadas na Seção 3.1.3. Os efeitos da gravidade são desprezados.

\subsubsection{Equações de Conservação de Massa e de Quantidade de Movi- mento}

Utilizando coordenadas cartesianas e a forma da velocidade (3.2), a equação de conservação de massa $(1.2)_{2}$ é escrita da seguinte forma

$$
u_{, x}+v_{, y}=0
$$

A densidade de energia elástica é calculada, utilizando a equação (1.9) e a forma geral do diretor (3.1), pela equação

$$
w_{F}=\frac{1}{2} K\left[\left(\phi_{, x}\right)^{2}+\left(\phi_{, y}\right)^{2}\right]
$$

As componentes da equação de conservação de quantidade de movimento (1.4) são dadas por (utilizando a equação (3.5) onde necessário),

$$
\begin{aligned}
\rho\left(u_{t}+\left(u^{2}\right)_{, x}+(u v)_{, y}\right) & =-p_{, x}-w_{F, x}+\tilde{g}_{j} n_{j, x}+G_{j} n_{j, x}+\tilde{t}_{x x, x}+\tilde{t}_{x y, y} \\
\rho\left(v_{t}+(u v)_{, y}+\left(v^{2}\right)_{, y}\right) & =-p_{, y}-w_{F, y}+\tilde{g}_{j} n_{j, y}+G_{j} n_{j, y}+\tilde{t}_{y x, x}+\tilde{t}_{y y, y}
\end{aligned}
$$

onde apenas o sufixo $j$ em $\tilde{g}_{j}$ e $G_{j}$ será somado. Após alguns cálculos envolvendo as expressões nas equações (1.12) à (1.15) obtém-se que

$$
\begin{aligned}
G_{j} n_{j, x} & =\frac{1}{2} \mu_{0} \Delta \chi H^{2} \phi_{, x} \operatorname{sen}\left(2\left(\phi_{0}-\phi\right)\right) \\
G_{j} n_{j, y} & =\frac{1}{2} \mu_{0} \Delta \chi H^{2} \phi_{, y} \operatorname{sen}\left(2\left(\phi_{0}-\phi\right)\right) .
\end{aligned}
$$


As componentes do vetor $\tilde{g}_{j}$ são dadas por:

$$
\begin{aligned}
\tilde{g}_{x}= & \gamma_{1} \operatorname{sen} \phi\left[\phi_{t}+u \phi_{, x}+v \phi_{, y}+\frac{1}{2}\left(u_{, y}-v_{, x}\right)\right] \\
& -\gamma_{2}\left[u_{, x} \cos \phi+\frac{1}{2} \operatorname{sen} \phi\left(u_{, y}+v_{, x}\right)\right] \\
\tilde{g}_{y}= & -\gamma_{1} \cos \phi\left[\phi_{t}+u \phi_{, x}+v \phi_{, y}+\frac{1}{2}\left(u_{, y}-v_{, x}\right)\right] \\
& -\gamma_{2}\left[v_{, y} \operatorname{sen} \phi+\frac{1}{2} \cos \phi\left(u_{, y}+v_{, x}\right)\right]
\end{aligned}
$$

e portanto,

$$
\begin{aligned}
\tilde{g}_{j} n_{j, x}= & -\gamma_{1} \phi_{, x}\left[\phi_{t}+u \phi_{, x}+v \phi_{, y}+\frac{1}{2}\left(u_{, y}-v_{, x}\right)\right] \\
& -\frac{1}{2} \gamma_{2} \phi_{, x} \cos (2 \phi)\left(u_{, y}+v_{, x}\right)+\frac{1}{2} \gamma_{2} \phi_{, x} \operatorname{sen}(2 \phi)\left(u_{, x}-v_{, y}\right), \\
\tilde{g}_{j} n_{j, y}= & -\gamma_{1} \phi_{, y}\left[\phi_{t}+u \phi_{, x}+v \phi_{, y}+\frac{1}{2}\left(u_{, y}-v_{, x}\right)\right] \\
& -\frac{1}{2} \gamma_{2} \phi_{, y} \cos (2 \phi)\left(u_{, y}+v_{, x}\right)+\frac{1}{2} \gamma_{2} \phi_{, y} \operatorname{sen}(2 \phi)\left(u_{, x}-v_{, y}\right) .
\end{aligned}
$$

As componentes do tensor das tensões viscosas $\tilde{t}_{i j}$ são dadas por

$$
\begin{aligned}
\tilde{t}_{x x}= & \alpha_{1} \cos ^{2} \phi\left[u_{, x} \cos ^{2} \phi+v_{, y} \operatorname{sen}^{2} \phi+\frac{1}{2}\left(u_{, y}+v_{, x}\right) \operatorname{sen}(2 \phi)\right] \\
& -\left(\alpha_{2}+\alpha_{3}\right) \operatorname{sen} \phi \cos \phi\left[\phi_{t}+u \phi_{, x}+v \phi_{, y}+\frac{1}{2}\left(u_{, y}-v_{, x}\right)\right]+\alpha_{4} u_{, x} \\
& +\left(\alpha_{5}+\alpha_{6}\right)\left[u_{, x} \cos ^{2} \phi+\frac{1}{2} \operatorname{sen} \phi \cos \phi\left(u_{, y}+v_{, x}\right)\right]
\end{aligned}
$$




$$
\begin{aligned}
& \tilde{t}_{x y}=\alpha_{1} \operatorname{sen} \phi \cos \phi\left[u_{, x} \cos ^{2} \phi+v_{, y} \operatorname{sen}^{2} \phi+\frac{1}{2}\left(u_{, y}+v_{, x}\right) \operatorname{sen}(2 \phi)\right] \\
& +\left(\alpha_{3} \cos ^{2} \phi-\alpha_{2} \operatorname{sen}^{2} \phi\right)\left[\phi_{t}+u \phi_{, x}+v \phi_{, y}+\frac{1}{2}\left(u_{, y}-v_{, x}\right)\right] \\
& +\frac{1}{2} \alpha_{4}\left(u_{, y}+v_{, x}\right)+\frac{1}{2}\left(\alpha_{5} \operatorname{sen}^{2} \phi+\alpha_{6} \cos ^{2} \phi\right)\left(u_{, y}+v_{, x}\right) \\
& +\left(\alpha_{5} u_{, x}+\alpha_{6} v_{, y}\right) \operatorname{sen} \phi \cos \phi \text {, } \\
& \tilde{t}_{y x}=\alpha_{1} \operatorname{sen} \phi \cos \phi\left[u_{, x} \cos ^{2} \phi+v_{, y} \operatorname{sen}^{2} \phi+\frac{1}{2}\left(u_{, y}+v_{, x}\right) \operatorname{sen}(2 \phi)\right] \\
& +\left(\alpha_{2} \cos ^{2} \phi-\alpha_{3} \operatorname{sen}^{2} \phi\right)\left[\phi_{t}+u \phi_{, x}+v \phi_{, y}+\frac{1}{2}\left(u_{, y}-v_{, x}\right)\right] \\
& +\frac{1}{2} \alpha_{4}\left(u_{, y}+v_{, x}\right)+\frac{1}{2}\left(\alpha_{5} \cos ^{2} \phi+\alpha_{6} \operatorname{sen}^{2} \phi\right)\left(u_{, y}+v_{, x}\right) \\
& +\left(\alpha_{5} v_{, y}+\alpha_{6} u_{, x}\right) \operatorname{sen} \phi \cos \phi \text {, } \\
& \tilde{t}_{y y}=\alpha_{1} \operatorname{sen}^{2} \phi\left[u_{, x} \cos ^{2} \phi+v_{, y} \operatorname{sen}^{2} \phi+\frac{1}{2}\left(u_{, y}+v_{, x}\right) \operatorname{sen}(2 \phi)\right] \\
& +\left(\alpha_{2}+\alpha_{3}\right) \operatorname{sen} \phi \cos \phi\left[\phi_{t}+u \phi_{, x}+v \phi_{, y}+\frac{1}{2}\left(u_{, y}-v_{, x}\right)\right]+\alpha_{4} v_{, y} \\
& +\left(\alpha_{5}+\alpha_{6}\right)\left[v_{, y} \operatorname{sen}^{2} \phi+\frac{1}{2} \operatorname{sen} \phi \cos \phi\left(u_{, y}+v_{, x}\right)\right] .
\end{aligned}
$$

Das equações acima, observa-se que $\tilde{t}_{i j} \neq \tilde{t}_{j i}$, e assim o tensor das tensões viscosas não é simétrico. Substituindo as expressões acima nas equações (3.7) e (3.8) obtém-se as equações dinâmicas, as quais foram originadas das equações de conservação de quantidade de movimento.

\subsubsection{Equações de Conservação de Momento Angular}

Sob as hipóteses antes mencionadas, adotando a aproximação constante para as constantes elásticas (1.8) e, usando a condição (1.10) e a forma geral do diretor (3.1), as equações de conservação de momento angular (1.5) podem ser escritas como:

$$
\begin{aligned}
K\left[(\cos \phi)_{, x x}+(\cos \phi)_{, y y}\right]+\tilde{g}_{x}+G_{x} & =\lambda \cos \phi \\
K\left[(\operatorname{sen} \phi)_{, x x}+(\operatorname{sen} \phi)_{, y y}\right]+\tilde{g}_{y}+G_{y} & =\lambda \operatorname{sen} \phi .
\end{aligned}
$$

Multiplicando (3.19) por sen $\phi$ e (3.20) por $\cos \phi$, e subtraindo uma da outra, obtém-se a equação 


$$
\begin{aligned}
\operatorname{sen} \phi\{K[ & \left.\left.(\cos \phi)_{, x x}+(\cos \phi)_{, y y}\right]+\tilde{g}_{x}+G_{x}\right\} \\
& -\cos \phi\left\{K\left[(\operatorname{sen} \phi)_{, x x}+(\operatorname{sen} \phi)_{, y y}\right]+\tilde{g}_{y}+G_{y}\right\}=0 .
\end{aligned}
$$

Agora, empregando as expressões (3.4), (3.11) e (3.12), e após efetuar alguns cálculos na equação (3.21), obtém-se a equação dinâmica para a conservação de momento angular

$$
\begin{aligned}
K\left(\phi_{, x x}+\right. & \left.\phi_{, y y}\right)=\gamma_{1}\left[\phi_{t}+u \phi_{, x}+v \phi_{, y}+\frac{1}{2}\left(u_{, y}-v_{, x}\right)\right] \\
& +\frac{1}{2} \gamma_{2}\left[\left(u_{, y}+v_{, x}\right) \cos (2 \phi)+\left(v_{, y}-u_{, x}\right) \operatorname{sen}(2 \phi)\right]+\frac{1}{2} \mu_{0} \Delta \chi H \operatorname{sen}\left(2\left(\phi-\phi_{0}\right)\right) .
\end{aligned}
$$

Resumindo, as equações (3.5), (3.7) e (3.8) devem ser resolvidas em conjunto com a equação (3.22) sujeitas à condições de contorno apropriadas para encontrar soluções para $u, v, p$ e $\phi$.

\subsubsection{Equações Dinâmicas Adimensionais}

Para a adimensionalização das equações, é seguida a metodologia apresentada em [133], e é empregada a seguinte mudança de variáveis

$$
\begin{gathered}
x_{i}=L \widehat{x}_{i}, \quad v_{i}=U \widehat{v}_{i}, \quad t=L U^{-1} \widehat{t}, \quad p=\rho U^{2} \widehat{p}, \quad \widetilde{t}_{i j}=\eta \widehat{S}_{i j}, \\
w_{F}=\rho U^{2} \widehat{w}_{F}, \quad \widetilde{g}_{j}=\rho U^{2} \widehat{R}_{j}, \quad G_{j}=\rho U^{2} \widehat{G}_{j},
\end{gathered}
$$

onde os fatores de escala para comprimento, velocidade e viscosidade, são denotados por $L, U$ e $\eta$, respectivamente. Neste trabalho utiliza-se o valor $\frac{1}{2} \alpha_{4}$ para a viscosidade de escala $\eta$.

Introduzindo essas variáveis nas equações (3.5), (1.9) e (1.4) obtém-se as seguintes equações na forma adimensional:

$$
\begin{aligned}
u_{, x} & +v_{, y}=0, \\
w_{F} & =\frac{1}{2} \frac{1}{\operatorname{ReEr}}\left[\left(\phi_{, x}\right)^{2}+\left(\phi_{, y}\right)^{2}\right], \\
u_{t} & +u u_{, x}+v u_{, y}=-p_{, x}-w_{F, x}+R_{j} n_{j, x}+G_{j} n_{j, x}+\frac{1}{R e}\left(S_{x x, x}+S_{x y, y}\right), \\
v_{t} & +u v_{, x}+v v_{, y}=-p_{, y}-w_{F, y}+R_{j} n_{j, y}+G_{j} n_{j, y}+\frac{1}{R e}\left(S_{y x, x}+S_{y y, y}\right),
\end{aligned}
$$

onde os circunflexos foram omitidos com o intuito de simplificar a notação e $R e=\frac{\rho U L}{\eta} \mathrm{e}$ $E r=U L \frac{\eta}{K}$ denotam os números de Reynolds e de Ericksen, respectivamente. Os termos 
$R_{j} n_{j, x}, R_{j} n_{j, y}, G_{j} n_{j, x}$ e $G_{j} n_{j, y}$ são dados por

$$
\begin{aligned}
& R_{j} n_{j, x}= \frac{1}{R e}\left\{-\gamma_{1} \phi_{, x}\left[\phi_{, t}+u \phi_{, x}+v \phi_{, y}+\frac{1}{2}\left(u_{, y}-v_{, x}\right)\right]\right. \\
&\left.-\frac{1}{2}\left[\gamma_{2} \phi_{, x} \cos (2 \phi)\left(u_{, y}+v_{, x}\right)+\gamma_{2} \phi_{, x} \operatorname{sen}(2 \phi)\left(u_{, x}-v_{, y}\right)\right]\right\}, \\
& R_{j} n_{j, y}= \frac{1}{R e}\left\{-\gamma_{1} \phi_{, y}\left[\phi_{, t}+u \phi_{, x}+v \phi_{, y}+\frac{1}{2}\left(u_{, y}-v_{, x}\right)\right]\right. \\
&\left.-\frac{1}{2}\left[\gamma_{2} \phi_{, y} \cos (2 \phi)\left(u_{, y}+v_{, x}\right)+\gamma_{2} \phi_{, y} \operatorname{sen}(2 \phi)\left(u_{, x}-v_{, y}\right)\right]\right\}, \\
& G_{j} n_{j, x}=\frac{1}{2} \mu_{0} \Delta \chi H^{2} \phi_{, x} \operatorname{sen}\left(2\left(\phi_{0}-\phi\right)\right), \\
& G_{j} n_{j, y}=\frac{1}{2} \mu_{0} \Delta \chi H^{2} \phi_{, y} \operatorname{sen}\left(2\left(\phi_{0}-\phi\right)\right) .
\end{aligned}
$$

e as componentes do tensor extra-tensão adimensional são dados por

$$
\begin{aligned}
& S_{x x}=\alpha_{1} \cos ^{2} \phi\left[u_{, x} \cos ^{2} \phi+v_{, y} \operatorname{sen}^{2} \phi+\frac{1}{2}\left(u_{, y}+v_{, x}\right) \operatorname{sen}(2 \phi)\right] \\
& -\left(\alpha_{2}+\alpha_{3}\right) \operatorname{sen} \phi \cos \phi\left[\phi_{t}+u \phi_{, x}+v \phi_{, y}+\frac{1}{2}\left(u_{, y}-v_{, x}\right)\right]+2 u_{, x} \\
& +\left(\alpha_{5}+\alpha_{6}\right)\left[u_{, x} \cos ^{2} \phi+\frac{1}{2} \operatorname{sen} \phi \cos \phi\left(u_{, y}+v_{, x}\right)\right], \\
& S_{x y}=\alpha_{1} \operatorname{sen} \phi \cos \phi\left[u_{, x} \cos ^{2} \phi+v_{, y} \operatorname{sen}^{2} \phi+\frac{1}{2}\left(u_{, y}+v_{, x}\right) \operatorname{sen}(2 \phi)\right] \\
& +\left(\alpha_{3} \cos ^{2} \phi-\alpha_{2} \operatorname{sen}^{2} \phi\right)\left[\phi_{t}+u \phi_{, x}+v \phi_{, y}+\frac{1}{2}\left(u_{, y}-v_{, x}\right)\right] \\
& +\left(u_{, y}+v_{, x}\right)+\frac{1}{2}\left(\alpha_{5} \operatorname{sen}^{2} \phi+\alpha_{6} \cos ^{2} \phi\right)\left(u_{, y}+v_{, x}\right) \\
& +\left(\alpha_{5} u_{, x}+\alpha_{6} v_{, y}\right) \operatorname{sen} \phi \cos \phi \text {, } \\
& S_{y x}=\alpha_{1} \operatorname{sen} \phi \cos \phi\left[u_{, x} \cos ^{2} \phi+v_{, y} \operatorname{sen}^{2} \phi+\frac{1}{2}\left(u_{, y}+v_{, x}\right) \operatorname{sen}(2 \phi)\right] \\
& +\left(\alpha_{2} \cos ^{2} \phi-\alpha_{3} \operatorname{sen}^{2} \phi\right)\left[\phi_{t}+u \phi_{, x}+v \phi_{, y}+\frac{1}{2}\left(u_{, y}-v_{, x}\right)\right] \\
& +\left(u_{, y}+v_{, x}\right)+\frac{1}{2}\left(\alpha_{5} \cos ^{2} \phi+\alpha_{6} \operatorname{sen}^{2} \phi\right)\left(u_{, y}+v_{, x}\right) \\
& +\left(\alpha_{5} v_{, y}+\alpha_{6} u_{, x}\right) \operatorname{sen} \phi \cos \phi \text {, }
\end{aligned}
$$




$$
\begin{aligned}
S_{y y}= & \alpha_{1} \operatorname{sen}^{2} \phi\left[u_{, x} \cos ^{2} \phi+v_{, y} \operatorname{sen}^{2} \phi+\frac{1}{2}\left(u_{, y}+v_{, x}\right) \operatorname{sen}(2 \phi)\right] \\
& +\left(\alpha_{2}+\alpha_{3}\right) \operatorname{sen} \phi \cos \phi\left[\phi_{t}+u \phi_{, x}+v \phi_{, y}+\frac{1}{2}\left(u_{, y}-v_{, x}\right)\right]+2 v_{, y} \\
& +\left(\alpha_{5}+\alpha_{6}\right)\left[v_{, y} \operatorname{sen}^{2} \phi+\frac{1}{2} \operatorname{sen} \phi \cos \phi\left(u_{, y}+v_{, x}\right)\right]
\end{aligned}
$$

Nas equações acima, as viscosidades $\alpha_{1}, \ldots, \alpha_{6}$ foram adimensionalizadas pelo fator $\eta$. A equação de conservação de momento angular (3.22) transforma-se na equação

$$
\begin{aligned}
\phi_{t}+u \phi_{, x}+v \phi_{, y} & =\frac{1}{E r \gamma_{1}}\left[\phi_{, x x}+\phi_{, y y}\right]-\frac{1}{2}\left(u_{, y}-v_{, x}\right) \\
& -\frac{1}{2} \frac{\gamma_{2}}{\gamma_{1}}\left[\left(u_{, y}+v_{, x}\right) \cos (2 \phi)+\left(v_{, y}-u_{, x}\right) \operatorname{sen}(2 \phi)\right]-\frac{1}{2} \frac{R e}{\gamma_{1}} \mu_{0} \Delta \chi H^{2} \operatorname{sen}\left(2\left(\phi_{0}-\phi\right)\right) .
\end{aligned}
$$

As equações (3.24), (3.26), (3.27) e (3.36) formam o conjunto de equações dinâmicas e devem ser resolvidas sujeitas à condições de contorno para encontrar soluções para $u, v, p$ e $\phi$.

\subsection{Método Numérico 2D}

Para resolver as equações (3.24), (3.26), (3.27) e (3.36) primeiramente escrevemos as componentes do tensor das tensões $S_{i j}$ como:

$$
\begin{aligned}
S_{x x} & =\left[2 u_{, x}+\Phi^{x x}\right], \\
S_{x y} & =\left[\left(u_{, y}+v_{, x}\right)+\Phi_{x y}\right], \\
S_{y x} & =\left[\left(u_{, y}+v_{, x}\right)+\Phi_{y x}\right], \\
S_{y y} & =\left[2 v_{, y}+\Phi_{y y}\right],
\end{aligned}
$$

onde as funções $\Phi_{x x}, \cdots, \Phi_{y y}$, são as componentes do tensor das tensões não-newtoniano $\boldsymbol{\Phi}$ dadas por

$$
\begin{aligned}
\Phi_{x x}= & \alpha_{1} \cos ^{2} \phi\left[u_{, x} \cos ^{2} \phi+v_{, y} \operatorname{sen}^{2} \phi+\frac{1}{2}\left(u_{, y}+v_{, x}\right) \operatorname{sen}(2 \phi)\right] \\
& -\left(\alpha_{2}+\alpha_{3}\right) \operatorname{sen} \phi \cos \phi\left[\phi_{t}+u \phi_{, x}+v \phi_{, y}+\frac{1}{2}\left(u_{, y}-v_{, x}\right)\right] \\
& +\left(\alpha_{5}+\alpha_{6}\right)\left[u_{, x} \cos ^{2} \phi+\frac{1}{2} \operatorname{sen} \phi \cos \phi\left(u_{, y}+v_{, x}\right)\right],
\end{aligned}
$$




$$
\begin{aligned}
& \Phi_{x y}=\alpha_{1} \operatorname{sen} \phi \cos \phi\left[u_{, x} \cos ^{2} \phi+v_{, y} \operatorname{sen}^{2} \phi+\frac{1}{2}\left(u_{, y}+v_{, x}\right) \operatorname{sen}(2 \phi)\right] \\
& +\left(\alpha_{3} \cos ^{2} \phi-\alpha_{2} \operatorname{sen}^{2} \phi\right)\left[\phi_{t}+u \phi_{, x}+v \phi_{, y}+\frac{1}{2}\left(u_{, y}-v_{, x}\right)\right] \\
& +\frac{1}{2}\left(\alpha_{5} \operatorname{sen}^{2} \phi+\alpha_{6} \cos ^{2} \phi\right)\left(u_{, y}+v_{, x}\right)+\left(\alpha_{5} u_{, x}+\alpha_{6} v_{, y}\right) \operatorname{sen} \phi \cos \phi, \\
& \Phi_{y x}=\alpha_{1} \operatorname{sen} \phi \cos \phi\left[u_{, x} \cos ^{2} \phi+v_{, y} \operatorname{sen}^{2} \phi+\frac{1}{2}\left(u_{, y}+v_{, x}\right) \operatorname{sen}(2 \phi)\right] \\
& +\left(\alpha_{2} \cos ^{2} \phi-\alpha_{3} \operatorname{sen}^{2} \phi\right)\left[\phi_{t}+u \phi_{, x}+v \phi_{, y}+\frac{1}{2}\left(u_{, y}-v_{, x}\right)\right] \\
& +\frac{1}{2}\left(\alpha_{5} \cos ^{2} \phi+\alpha_{6} \operatorname{sen}^{2} \phi\right)\left(u_{, y}+v_{, x}\right)+\left(\alpha_{5} v_{, y}+\alpha_{6} u_{, x}\right) \operatorname{sen} \phi \cos \phi, \\
& \Phi_{y y}=\alpha_{1} \operatorname{sen}^{2} \phi\left[u_{, x} \cos ^{2} \phi+v_{, y} \operatorname{sen}^{2} \phi+\frac{1}{2}\left(u_{, y}+v_{, x}\right) \operatorname{sen}(2 \phi)\right] \\
& +\left(\alpha_{2}+\alpha_{3}\right) \operatorname{sen} \phi \cos \phi\left[\phi_{t}+u \phi_{, x}+v \phi_{, y}+\frac{1}{2}\left(u_{, y}-v_{, x}\right)\right] \\
& +\left(\alpha_{5}+\alpha_{6}\right)\left[v_{, y} \operatorname{sen}^{2} \phi+\frac{1}{2} \operatorname{sen} \phi \cos \phi\left(u_{, y}+v_{, x}\right)\right] .
\end{aligned}
$$

Assim, as equações de conservação de quantidade de movimento (3.26) e (3.27) podem ser escritas na forma adimensional

$$
\begin{aligned}
u_{t}+u u_{, x}+v u_{, y}= & -p_{, x}-w_{F, x}+R_{j} n_{j, x}+G_{j} n_{j, x} \\
& +\frac{1}{R e}\left[u_{, x x}+u_{, y y}\right]+\frac{1}{R e}\left[\Phi_{x x, x}+\Phi_{x y, y}\right], \\
v_{t}+u v_{, x}+v v_{, y}= & -p_{, y}-w_{F, y}+R_{j} n_{j, y}+G_{j} n_{j, y} \\
& +\frac{1}{R e}\left[v_{, x x}+v_{, y y}\right]+\frac{1}{R e}\left[\Phi_{y x, x}+\Phi_{y y, y}\right]
\end{aligned}
$$

e a equação de conservação de massa (3.24) permanece inalterada.

Portanto, para simular o escoamento de um cristal líquido nemático para um valor finito do campo magnético $H$, temos que resolver as equações (3.24), (3.45), (3.46) em conjunto com (3.36) para encontrar soluções para as componentes da velocidade $u, v, p$ e para o ângulo de orientação do diretor $\phi$, junto com as equações $(3.41)-(3.44)$ para as variáveis $\Phi_{x x}, \cdots, \Phi_{y y}$, respectivamente. 


\subsubsection{Condições de Contorno}

Para resolver as equações (3.24), (3.45), (3.46) e (2.37) é necessário impor condições de contorno para o campo de velocidades como segue.

- Para contornos rígidos emprega-se a condição de não deslizamento $u_{i}=0$.

- Para entrada e saídas de fluido, os valores da velocidade são especificados como segue:

i) $\mathrm{Na}$ entrada de fluido a velocidade normal é prescrita como $u_{\nu}=U_{\text {inf }}$ enquanto que a velocidade tangencial é imposta com valor nulo, ou seja, $u_{\mu}=0$, onde $\nu$ denota a direção normal ao inflow e $\mu$ denota a direção tangencial ao inflow.

ii) Na saída de fluido impõe-se a condição homogênea de Neumann $u_{i, \nu}=0$.

O diretor é fortemente ancorado nos contornos rígidos. Em outras palavras, o ângulo de ancoragem é atribuído de acordo com a orientação do contorno rígido. Detalhes do ângulo de ancoragem do diretor são dados nos Capítulos que tratam dos resultados numéricos.

A escolha do ângulo do diretor na entrada de fluidos é $\phi=0$, e na saída de fluidos atribuímos $\phi_{, \nu}=0$.

\subsubsection{Procedimento Numérico 2D}

As equações de conservação de quantidade de movimento (3.45)-(3.46), a equação de conservação de massa (3.24) e a equação de conservação de momento angular (3.36) são resolvidas por uma metodologia baseada no algoritmo GENSMAC introduzido por Tomé et al. [133] como segue.

Assume-se que, no instante de tempo $t_{n}$, o campo de velocidades $u_{i}\left(x_{k}, t_{n}\right)$ e o ângulo de orientação do diretor $\phi\left(x_{k}, t_{n}\right)$ são conhecidos e que as condições de contorno para a velocidade e pressão são conhecidas. Para calcular o campo de velocidades $u_{i}\left(x_{k}, t_{n+1}\right)$, a pressão $p\left(x_{k}, t_{n+1}\right)$, o tensor não-newtoniano $\Phi_{i j}\left(x_{k}, t_{n+1}\right)$ e o ângulo de orientação do diretor $\phi\left(x_{k}, t_{n+1}\right)$, procedemos da seguinte maneira:

Passo 1: Utilizando os valores de $u_{i}\left(x_{k}, t_{n}\right)$ e $\phi\left(x_{k}, t_{n}\right)$, resolve-se a equação (3.25) para $w_{F}\left(x_{k}, t_{n}\right)$ e calcula-se $w_{F, i}\left(x_{k}, t_{n}\right), \Phi_{i j}\left(x_{k}, t_{n}\right), R_{j} n_{j, i}\left(x_{k}, t_{n}\right), G_{j} n_{j, i}\left(x_{k}, t_{n}\right)$ das equações (3.41)(3.44) e (3.28)-(3.31), respectivamente.

Passo 2: Calcula-se o campo de velocidade intermediária $\widetilde{u}_{i}\left(x_{k}, t_{n+1}\right)$ por

$$
\frac{\partial \widetilde{u}_{i}}{\partial t}=-\left(u_{j} u_{i}\right), j_{j}-w_{F, i}+R_{j} n_{j, i}+G_{j} n_{j, i}+\frac{1}{R e}\left[\left(u_{i, j}\right)_{, j}+\Phi_{i j, j}\right]
$$

com $\widetilde{u}_{i}\left(x_{k}, t_{n}\right)=u_{i}\left(x_{k}, t_{n}\right)$ utilizando as mesmas condições de contorno para a velocidade $u_{i}\left(x_{k}, t_{n}\right)$. Esta equação é resolvida por um método explícito de diferenças finitas. Pode ser 
mostrado que $\widetilde{u}_{i}$ possui a vorticidade correta no tempo $t_{n+1}$ (ver Tomé et al. [131]).

Passo 3: Resolve-se a equação de Poisson

$$
\psi_{, i i}\left(x_{k}, t_{n+1}\right)=\widetilde{u}_{i, i}\left(x_{k}, t_{n+1}\right)
$$

sujeita as condições de contorno (ver Tomé e McKee [134]): $\psi_{, \nu}=0$ nos contornos rígidos e entradas de fluido e $\psi=0$ nas saídas de fluido.

Passo 4: Calcula-se o campo de velocidades final

$$
u_{i}\left(x_{k}, t_{n+1}\right)=\widetilde{u}_{i}\left(x_{k}, t_{n+1}\right)-\psi_{, i}\left(x_{k}, t_{n+1}\right) .
$$

Passo 5: Determina-se o campo de pressões $p\left(x_{k}, t_{n+1}\right)$ (ver Tomé et al. [131])

$$
p\left(x_{k}, t_{n+1}\right)=\frac{\psi\left(x_{k}, t_{n+1}\right)}{\delta t}
$$

Passo 6: Calcula-se o ângulo do diretor $\phi\left(x_{k}, t_{n+1}\right)$ da equação (3.36). Esta equação é resolvida por um método explícito de diferenças finitas.

Passo 7: Calcula-se os componentes do tensor não newtoniano $\Phi_{i j}\left(x_{k}, t_{n+1}\right)$ por meio das equações (3.41)-(3.44).

\subsection{Aproximação das Equações por Diferenças Finitas}

As equações contidas no procedimento numérico apresentado na seção anterior são resolvidas pelo método de diferenças finitas como segue.

As velocidades $u$ e $v$ estão localizadas no meio das faces das células enquanto que as outras quantidades $\left(\phi, \Phi, p, w_{F}\right)$ estão posicionadas no centro das células. A Figura 3.1 ilustra uma célula bidimensional de dimensões $\delta x \times \delta y$. As equações de conservação de quantidade de movimento (3.45)-(3.46) e a equação de conservação de momento angular (3.36) são resolvidas pelo método de Euler explícito. As derivadas espaciais na equação de conservação de quantidade de movimento são discretizadas nos pontos $\left(\left(i+\frac{1}{2}\right) \delta x, j \delta y\right)$ e $\left(i \delta x,\left(j+\frac{1}{2}\right) \delta y\right)$ enquanto a equação de conservação de momento angular (3.36), a densidade de energia elástica (3.25) e o tensor não newtoniano $\Phi_{i j}$ são calculados nos centros das células $(i \delta x, j \delta y)$.

O gradiente de pressão, o divergente do tensor das tensões não-newtoniano, o gradiente da densidade de energia elástica, a força externa e os termos $R_{j} n_{j, x}, R_{j} n_{j, y}$ são aproximados por diferenças centrais. 


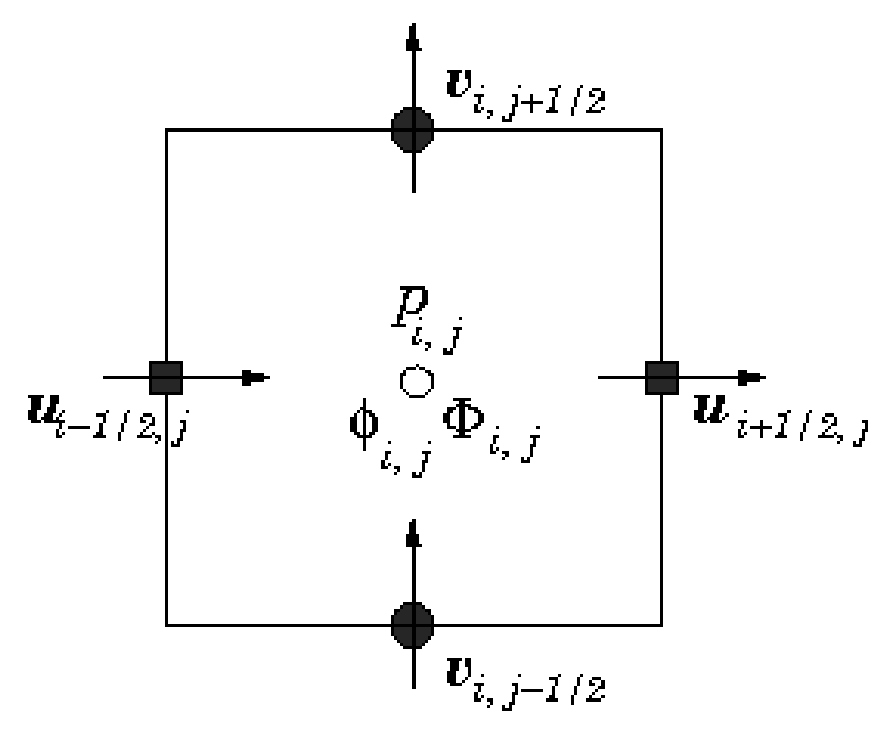

Figura 3.1: Típica célula deslocada bidimensional.

Em resumo, a equação de conservação de momento angular e de quantidade de movimento são aproximadas pelas seguintes equações de diferenças finitas:

$$
\begin{aligned}
\phi_{i, j}\left(t_{n+1}\right)= & \phi_{i, j}\left(t_{n}\right)+\delta t\left\{\frac{1}{E r \gamma_{1}}\left[\left(\frac{\phi_{i-1, j}-2 \phi_{i, j}+\phi_{i+1, j}}{\delta x^{2}}\right)+\left(\frac{\phi_{i, j-1}-2 \phi_{i, j}+\phi_{i, j+1}}{\delta y^{2}}\right)\right]\right. \\
& -\left.\mathcal{C}(\phi)\right|_{i, j}-\left.\frac{1}{2}\left(u_{, y}-v_{, x}\right)\right|_{i, j}-\frac{1}{2} \frac{\gamma_{2}}{\gamma_{1}}\left[\left.\left(u_{, y}+v_{, x}\right)\right|_{i, j} \cos \left(2 \phi_{i, j}\right)\right. \\
& \left.\left.+\left.\left(v_{, y}-u_{, x}\right)\right|_{i, j} \operatorname{sen}\left(2 \phi_{i, j}\right)\right]-\frac{1}{2} \frac{R e}{\gamma_{1}} \mu_{0} \Delta \chi H^{2} \operatorname{sen}\left(2\left(\phi_{0}-\phi_{i, j}\right)\right)\right\}_{t=t_{n}}, \\
\widetilde{u}_{i+1 / 2, j}= & u_{i+1 / 2, j}+\delta t\left\{-\left.\mathcal{C}(u)\right|_{i+1 / 2, j}-\left.w_{F, x}\right|_{i+1 / 2, j}+\left.R_{j} n_{j, x}\right|_{i+1 / 2, j}+\left.G_{j} n_{j, x}\right|_{i+1 / 2, j}\right. \\
+ & \frac{1}{R e}\left[\left(\frac{u_{i-1 / 2, j}-2 u_{i+1 / 2, j}+u_{i+3 / 2, j}}{\left.\delta x^{2}+\frac{u_{i+1 / 2, j-1}-2 u_{i+1 / 2, j}+u_{i+1 / 2, j+1}}{\delta y^{2}}\right)}\right)\right. \\
+ & \left.\left.\left(\frac{\Phi_{i+1, j}^{x x}-\Phi_{i, j}^{x x}}{\delta x}\right)+\left(\frac{\Phi_{i+1 / 2, j+1 / 2}^{x y}-\Phi_{i+1 / 2, j-1 / 2}^{x y}}{\delta y}\right)\right]\right\}_{t=t_{n}} \\
\widetilde{v}_{i, j+1 / 2} & v_{i, j+1 / 2}+\delta t\left\{-\left.\mathcal{C}(v)\right|_{i, j+1 / 2}-\left.\widetilde{w}_{F, x}\right|_{i, j+1 / 2}+\left.R_{j} n_{j, x}\right|_{i, j+1 / 2}+\left.\widetilde{G}_{j} n_{j, x}\right|_{i, j+1 / 2}\right. \\
+ & \frac{1}{R e}\left[\left(\frac{v_{i-1, j+1 / 2}-2 v_{i, j+1 / 2}+v_{i+1, j+1 / 2}}{\delta x^{2}}+\frac{v_{i, j-1 / 2}-2 v_{i, j+1 / 2}+v_{i, j+3 / 2}}{\delta y^{2}}\right)\right. \\
+ & \left.\left.\left(\frac{\Phi_{i, j+1}^{y y}-\Phi_{i, j}^{y y}}{\delta y}\right)\left(\frac{\Phi_{i+1 / 2, j+1 / 2}^{y x}-\Phi_{i-1 / 2, j+1 / 2}^{y x}}{\delta x}\right)\right]\right\}_{t=t_{n}}
\end{aligned}
$$

onde $\Phi_{i, j}^{l m}$ são as componentes do tensor das tensões não newtoniano. Por exemplo, o termo $\left.R_{j} n_{j, x}\right|_{i+1 / 2, j}$ é calculado por 


$$
\begin{aligned}
\left.R_{j} n_{j, x}\right|_{i+1 / 2, j}= & \frac{1}{R e}\left\{-\left.\gamma_{1} \phi_{, x}\right|_{i+1 / 2, j}\left[\left.\phi_{, t}\right|_{i+1 / 2, j}+\left.\mathcal{C}(\phi)\right|_{i+1 / 2, j}+\frac{1}{2}\left(\left.u_{, y}\right|_{i+1 / 2, j}-\left.v_{, x}\right|_{i+1 / 2, j}\right)\right]\right. \\
& -\frac{1}{2}\left[\left.\gamma_{2} \phi_{, x}\right|_{i+1 / 2, j} \cos \left(\left.2 \phi\right|_{i+1 / 2, j}\right)\left(\left.u_{, y}\right|_{i+1 / 2, j}+\left.v_{, x}\right|_{i+1 / 2, j}\right)\right. \\
& \left.\left.+\left.\gamma_{2} \phi_{, x}\right|_{i+1 / 2, j} \operatorname{sen}\left(\left.2 \phi\right|_{i+1 / 2, j}\right)\left(\left.u_{, x}\right|_{i+1 / 2, j}-\left.v_{, y}\right|_{i+1 / 2, j}\right)\right]\right\} .
\end{aligned}
$$

As derivadas na equação acima são aproximadas por

$$
\begin{aligned}
\left.\phi_{, x}\right|_{i+1 / 2, j} & =\left(\phi_{i+1, j}-\phi_{i, j}\right) / \delta x,\left.\quad u_{, x}\right|_{i+1 / 2, j}=\left(u_{i+}-u_{i-}\right) / \delta x \\
\left.v_{, x}\right|_{i+1 / 2, j} & =\left(v_{i+}-v_{i-}\right) / \delta x
\end{aligned}
$$

em que

$$
\begin{array}{ll}
u_{i+}=0.5 *\left(u_{i+1 / 2, j}+u_{i+3 / 2, j}\right), & u_{i-}=0.5 *\left(u_{i-1 / 2, j}+u_{i+1 / 2, j}\right), \\
v_{i+}=0.5 *\left(v_{i+1, j+1 / 2}+v_{i+1, j-1 / 2}\right), & v_{i-}=0.5 *\left(v_{i, j+1 / 2}+v_{i, j-1 / 2}\right) .
\end{array}
$$

Os outros termos, $\left.R_{j} n_{j, y}\right|_{i, j+1 / 2},\left.G_{j} n_{j, x}\right|_{i+1 / 2, j},\left.G_{j} n_{j, y}\right|_{i, j+1 / 2}$, são calculados de forma similar. Os termos $\left.\mathcal{C}(u)\right|_{i+1 / 2, j},\left.\mathcal{C}(v)\right|_{i, j+1 / 2},\left.\mathcal{C}(\phi)\right|_{i, j},\left.\mathcal{C}(\phi)\right|_{i+1 / 2, j}$ e $\left.\mathcal{C}(\phi)\right|_{i, j+1 / 2}$ representam a discretização dos termos convectivos utilizando o método CUBISTA (ver Alves et al. [1]). Detalhes específicos da discretização fornecida pelo método CUBISTA pode ser encontrado em Ferreira et al. [50]. Mais detalhes das equações de diferenças finitas obtidas podem ser encontrados em Cruz et al. [32]. Nas equações de conservação de quantidade de movimento (3.52) e (3.53), termos como $\Phi_{i+1 / 2, j+1 / 2}^{x y}$ e $\Phi_{i+1 / 2, j+1 / 2}^{y x}$ que não são definidos em seus respectivos nós são obtidos tomando a média aritmética dos nós vizinhos como segue:

$$
\Phi_{i+1 / 2, j+1 / 2}^{x y}=\frac{\Phi_{i, j}^{x y}+\Phi_{i+1, j}^{x y}+\Phi_{i, j+1}^{x y}+\Phi_{i+1, j+1}^{x y}}{4} .
$$

A equação de Poisson dada por (3.50) é discretizada no centro da célula utilizando o operador laplaciano o qual fornece a seguinte equação de diferenças:

$$
\frac{\psi_{i+1, j}-2 \psi_{i, j}+\psi_{i-1, j}}{\delta x^{2}}+\frac{\psi_{i, j+1}-2 \psi_{i, j}+\psi_{i, j-1}}{\delta y^{2}}=\tilde{D}_{i, j}
$$

onde

$$
\tilde{D}_{i, j}=\frac{\tilde{u}_{i+1 / 2, j}-\tilde{u}_{i-1 / 2, j}}{\delta x}+\frac{\tilde{v}_{i, j+1 / 2}-\tilde{v}_{i, j-1 / 2}}{\delta y}
$$


A equação (3.55) resulta em um sistema linear de equações, cuja matriz associada é positiva definida. Este sistema resultante é então resolvido eficientemente (sem a necessidade de pré-condicionadores) pelo método dos gradientes conjugados.

As componentes da velocidade $u$ e $v$ no tempo $t_{n+1}$ são então obtidas pela discretização da equação (3.49) em seus respectivos nós:

$$
\begin{aligned}
& u_{i+1 / 2, j}^{(n+1)}=\tilde{u}_{i+1 / 2, j}-\left(\frac{\psi_{i+1, j}-\psi_{i, j}}{\delta x}\right), \\
& v_{i, j+1 / 2}^{(n+1)}=\tilde{v}_{i, j+1 / 2}-\left(\frac{\psi_{i, j+1}-\psi_{i, j}}{\delta y}\right) .
\end{aligned}
$$

A pressão é dada por (ver Tomé et al. [131])

$$
p_{i, j}=\frac{\psi_{i, j}}{\delta t}
$$

\subsubsection{Aproximação do Tensor das Tensões não-newtoniano}

As componentes do tensor das tensões não-newtoniano, dadas pelas equações (3.41)-(3.44), são aproximadas por diferenças centrais. Os termos envolvendo as derivadas cruzadas como por exemplo $u_{, y}$ e $v_{, x}$ são obtidos como segue:

$$
\left.u_{, y}\right|_{i, j}=\frac{u_{i, j+1 / 2}-u_{i, j-1 / 2}}{\delta y},\left.\quad v_{, x}\right|_{i, j}=\frac{v_{i+1 / 2, j}-v_{i-1 / 2, j}}{\delta x}
$$

em que

$$
\begin{aligned}
& u_{i, j+1 / 2}=0.25\left(u_{i+1 / 2, j}+u_{i-1 / 2, j}+u_{i+1 / 2, j+1}+u_{i-1 / 2, j+1}\right), \\
& u_{i, j-1 / 2}=0.25\left(u_{i+1 / 2, j}+u_{i-1 / 2, j}+u_{i+1 / 2, j-1}+u_{i-1 / 2, j-1}\right), \\
& v_{i+1 / 2, j}=0.25\left(v_{i, j+1 / 2}+v_{i, j-1 / 2}+v_{i+1, j+1 / 2}+v_{i+1, j-1 / 2}\right), \\
& v_{i-1 / 2, j}=0.25\left(v_{i, j+1 / 2}+v_{i, j-1 / 2}+v_{i-1, j+1 / 2}+v_{i-1, j-1 / 2}\right) .
\end{aligned}
$$

Finalmente, as equações para as componentes do tensor das tensões não newtoniano (3.41)-(3.44) são aproximados por 


$$
\begin{aligned}
& \Phi_{i, j}^{x x}=\alpha_{1} \cos ^{2} \phi_{i, j}\left[\frac{u_{i+1 / 2, j}-u_{i-1 / 2, j}}{\delta x} \cos ^{2} \phi_{i, j}+\frac{v_{i, j+1 / 2}-v_{i, j-1 / 2}}{\delta y} \operatorname{sen}^{2} \phi_{i, j}\right. \\
& \left.+\frac{1}{2}\left(\frac{u_{i, j+1 / 2}-u_{i, j-1 / 2}}{\delta y}+\frac{v_{i+1 / 2, j}-v_{i-1 / 2, j}}{\delta x}\right) \operatorname{sen}\left(2 \phi_{i, j}\right)\right] \\
& -\left(\alpha_{2}+\alpha_{3}\right) \operatorname{sen} \phi_{i, j} \cos \phi_{i, j}\left[\left(\frac{\widetilde{\phi}_{i, j}-\phi_{i, j}}{\delta t}\right)+\left.\mathcal{C}(\phi)\right|_{i, j}\right. \\
& \left.+\frac{1}{2}\left(\frac{u_{i, j+1 / 2}-u_{i, j-1 / 2}}{\delta y}+\frac{v_{i+1 / 2, j}-v_{i-1 / 2, j}}{\delta x}\right)\right] \\
& +\left(\alpha_{5}+\alpha_{6}\right)\left[\frac{u_{i+1 / 2, j}-u_{i-1 / 2, j}}{\delta x} \cos ^{2} \phi_{i, j}\right. \\
& \left.+\frac{1}{2} \operatorname{sen} \phi_{i, j} \cos \phi_{i, j}\left(\frac{u_{i, j+1 / 2}-u_{i, j-1 / 2}}{\delta y}+\frac{v_{i+1 / 2, j}-v_{i-1 / 2, j}}{\delta x}\right)\right], \\
& \Phi_{i, j}^{x y}=\alpha_{1} \operatorname{sen} \phi_{i, j} \cos \phi_{i, j}\left[\frac{u_{i+1 / 2, j}-u_{i-1 / 2, j}}{\delta x} \cos ^{2} \phi_{i, j}+\frac{v_{i, j+1 / 2}-v_{i, j-1 / 2}}{\delta y} \operatorname{sen}^{2} \phi_{i, j}\right. \\
& \left.+\frac{1}{2}\left(\frac{u_{i, j+1 / 2}-u_{i, j-1 / 2}}{\delta y}+\frac{v_{i+1 / 2, j}-v_{i-1 / 2, j}}{\delta x}\right) \operatorname{sen}\left(2 \phi_{i, j}\right)\right] \\
& +\operatorname{sen} \phi_{i, j} \cos \phi_{i, j}\left(\alpha_{5} \frac{u_{i+1 / 2, j}-u_{i-1 / 2, j}}{\delta x}+\alpha_{6} \frac{v_{i, j+1 / 2}-v_{i, j-1 / 2}}{\delta y}\right) \\
& +\frac{1}{2}\left(\alpha_{6} \cos ^{2} \phi_{i, j}+\alpha_{5} \operatorname{sen}^{2} \phi_{i, j}\right)\left(\frac{u_{i, j+1 / 2}-u_{i, j-1 / 2}}{\delta y}+\frac{v_{i+1 / 2, j}-v_{i-1 / 2, j}}{\delta x}\right) \\
& +\left(\alpha_{3} \cos ^{2} \phi_{i, j}-\alpha_{2} \operatorname{sen}^{2} \phi_{i, j}\right)\left[\left(\frac{\widetilde{\phi}_{i, j}-\phi_{i, j}}{\delta t}\right)+\left.\mathcal{C}(\phi)\right|_{i, j}\right. \\
& \left.+\frac{1}{2}\left(\frac{u_{i, j+1 / 2}-u_{i, j-1 / 2}}{\delta y}-\frac{v_{i+1 / 2, j}-v_{i-1 / 2, j}}{\delta x}\right)\right] \text {, }
\end{aligned}
$$




$$
\begin{aligned}
& \Phi_{i, j}^{y x}=\alpha_{1} \operatorname{sen} \phi_{i, j} \cos \phi_{i, j}\left[\frac{u_{i+1 / 2, j}-u_{i-1 / 2, j}}{\delta x} \cos ^{2} \phi_{i, j}+\frac{v_{i, j+1 / 2}-v_{i, j-1 / 2}}{\delta y} \operatorname{sen}^{2} \phi_{i, j}\right. \\
& \left.+\frac{1}{2}\left(\frac{u_{i, j+1 / 2}-u_{i, j-1 / 2}}{\delta y}+\frac{v_{i+1 / 2, j}-v_{i-1 / 2, j}}{\delta x}\right) \operatorname{sen}\left(2 \phi_{i, j}\right)\right] \\
& +\operatorname{sen} \phi_{i, j} \cos \phi_{i, j}\left(\alpha_{6} \frac{u_{i+1 / 2, j}-u_{i-1 / 2, j}}{\delta x}+\alpha_{5} \frac{v_{i, j+1 / 2}-v_{i, j-1 / 2}}{\delta y}\right) \\
& +\frac{1}{2}\left(\alpha_{5} \cos ^{2} \phi_{i, j}+\alpha_{6} \operatorname{sen}^{2} \phi_{i, j}\right)\left(\frac{u_{i, j+1 / 2}-u_{i, j-1 / 2}}{\delta y}+\frac{v_{i+1 / 2, j}-v_{i-1 / 2, j}}{\delta x}\right) \\
& +\left(\alpha_{2} \cos ^{2} \phi_{i, j}-\alpha_{3} \operatorname{sen}^{2} \phi_{i, j}\right)\left[\left(\frac{\widetilde{\phi}_{i, j}-\phi_{i, j}}{\delta t}\right)+\left.\mathcal{C}(\phi)\right|_{i, j}\right. \\
& \left.+\frac{1}{2}\left(\frac{u_{i, j+1 / 2}-u_{i, j-1 / 2}}{\delta y}-\frac{v_{i+1 / 2, j}-v_{i-1 / 2, j}}{\delta x}\right)\right] \text {, } \\
& \Phi_{i, j}^{y y}=\alpha_{1} \operatorname{sen}^{2} \phi_{i, j}\left[\frac{u_{i+1 / 2, j}-u_{i-1 / 2, j}}{\delta x} \cos ^{2} \phi_{i, j}+\frac{v_{i, j+1 / 2}-v_{i, j-1 / 2}}{\delta y} \operatorname{sen}^{2} \phi_{i, j}\right. \\
& \left.+\frac{1}{2}\left(\frac{u_{i, j+1 / 2}-u_{i, j-1 / 2}}{\delta y}+\frac{v_{i+1 / 2, j}-v_{i-1 / 2, j}}{\delta x}\right) \operatorname{sen}\left(2 \phi_{i, j}\right)\right] \\
& +\left(\alpha_{2}+\alpha_{3}\right) \operatorname{sen} \phi_{i, j} \cos \phi_{i, j}\left[\left(\frac{\widetilde{\phi}_{i, j}-\phi_{i, j}}{\delta t}\right)+\left.\mathcal{C}(\phi)\right|_{i, j}+\frac{1}{2}\left(\frac{u_{i, j+1 / 2}-u_{i, j-1 / 2}}{\delta y}\right.\right. \\
& \left.\left.-\frac{v_{i+1 / 2, j}-v_{i-1 / 2, j}}{\delta x}\right)\right]+\left(\alpha_{5}+\alpha_{6}\right)\left[\frac{v_{i, j+1 / 2}-v_{i, j-1 / 2}}{\delta y} \operatorname{sen}^{2} \phi_{i, j}\right. \\
& \left.+\frac{1}{2} \operatorname{sen} \phi_{i, j} \cos \phi_{i, j}\left(\frac{u_{i, j+1 / 2}-u_{i, j-1 / 2}}{\delta y}+\frac{v_{i+1 / 2, j}-v_{i-1 / 2, j}}{\delta x}\right)\right] .
\end{aligned}
$$

\subsection{Cálculo do Passo Temporal}

Em cada ciclo de cálculo, o método GENSMAC [134] determina o tamanho do passo temporal, a cada tempo $t_{n}$, que é calculado segundo as seguintes restrições de estabilidade:

$$
\begin{aligned}
\delta t_{x} & \leq \frac{\delta x}{|u|_{\text {max }}} \\
\delta t_{y} & \leq \frac{\delta y}{|v|_{\max }}
\end{aligned}
$$

onde $|u|_{\max }$ e $|v|_{\max }$ são os módulos máximos das velocidades nas direções $x$ e $y$ respectivamente. Essa restrição garante que nenhuma partícula possa cruzar mais do que uma célula em um dado intervalo de tempo.

Devido à discretização explícita da equação de conservação de quantidade de movimento, 
adota-se a seguinte restrição de estabilidade que envolve o número de Reynolds e a viscosidade

$$
\delta t_{v i s c} \leq \frac{R e}{2}\left(\frac{1}{(\delta x)^{2}}+\frac{1}{(\delta y)^{2}}\right)^{-1}
$$

O passo temporal escolhido é

$$
\delta t=\min \left\{\delta t_{x}, \delta t_{y}, \delta t_{v i s c}\right\}
$$

A implementação dessas restrições para determinar o tamanho do passo temporal seguem as mesmas ideias de Tomé e McKee [134].

\subsection{Validação do Método Numérico Bidimensional}

As equações de diferenças finitas correspondentes ao método numérico apresentado na Seção 3.3 foram incorporadas ao sistema de simulação Freeflow2D [42] para simular escoamentos bidimensionais de cristais líquidos nemáticos governados pelas equações dinâmicas de Ericksen-Leslie.

Para validar a implementação desse método numérico, considera-se o escoamento em um canal bidimensional. Recentemente, Cruz et al. [34] encontraram uma solução analítica para escoamento totalmente desenvolvido em um canal bidimensional. Para obter a solução analítica para este problema foram impostas as hipóteses de escoamento totalmente desenvolvido e, na ausência de torques externos (ver Carou et. al. [19, 20, 21]), foi considerado que o ângulo de orientação do diretor é pequeno. Nessas condições, o sistema de equações resultantes foi resolvido analiticamente e obteve-se expressões para a velocidade $u$, para o ângulo de orientação do diretor $\phi$ e consequentemente, obteve-se expressões para as componentes do tensor das tensões não-newtoniano $\Phi_{i j}$. Detalhes de como essa solução analítica foi obtida são apresentados a seguir (para mais detalhes ver [34] ).

\subsubsection{Solução Analítica para Escoamentos Desenvolvidos 2D}

Considere o escoamento de um cristal líquido nemático em um canal bidimensional, como apresentado na Figura 3.2.

Em geral, as equações governantes para este tipo de escoamento são equações diferenciais acopladas que envolvem funções não lineares de $\phi$, o que dificulta a obtenção de soluções exatas para as mesmas. Entretanto, uma solução analítica pode ser obtida como segue.

Assume-se escoamento completamente desenvolvido de modo que as seguintes condições são válidas

$$
\frac{\partial}{\partial t} \equiv \frac{\partial}{\partial x} \equiv 0, \quad v=0, \quad u=u(y) \quad \text { e } \quad p_{, x}=\text { constante } .
$$

Portanto, a equação de conservação de massa é satisfeita e introduzindo essas hipóteses nas equações de conservação de quantidade de movimento e de conservação de momento angular 


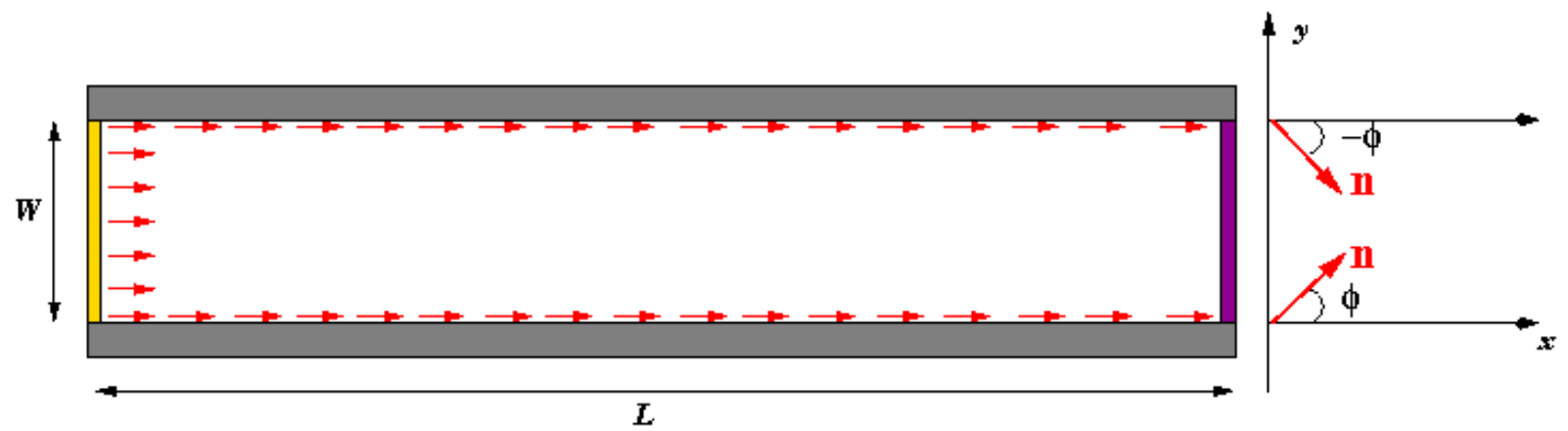

Figura 3.2: Descrição do escoamento em um Canal 2D. As setas vermelhas representam as condições de contorno utilizadas para o cálculo do ângulo $\phi$.

(3.26), (3.27) e (3.36), obtém-se as seguintes equações

$$
\begin{aligned}
0 & =-p_{, x}+\frac{1}{R e}\left(u_{, y}[1+h(\phi)]\right)_{, y}, \\
0 & =-\left(p+w_{F}\right)_{, y}+R_{j} n_{j, y}+G_{j} n_{j, y}+\frac{1}{R e} S_{y y, y}, \\
0 & =\phi_{, y y}-\frac{1}{2} u_{, y} \operatorname{Er}\left(\gamma_{1}+\gamma_{2} \cos (2 \phi)\right)+\frac{1}{2} \operatorname{Re} \operatorname{Er} \mu_{0} \Delta \chi H^{2} \operatorname{sen}\left(2\left(\phi-\phi_{0}\right)\right),
\end{aligned}
$$

em que

$$
h(\phi)=\alpha_{1} \operatorname{sen}^{2} \phi \cos ^{2} \phi+\frac{1}{2}\left(\alpha_{3}+\alpha_{6}\right) \cos ^{2} \phi+\frac{1}{2}\left(\alpha_{5}-\alpha_{2}\right) \operatorname{sen}^{2} \phi .
$$

Note que, na equação de conservação de momento angular simplificada (3.68), o termo

$$
\frac{1}{2} \operatorname{Re} \operatorname{Er} \mu_{0} \Delta \chi H \operatorname{sen}\left(2\left(\phi-\phi_{0}\right)\right) \approx O\left(10^{-11}\right)
$$

e portanto, esse termo pode ser desprezado.

Equações (3.66)-(3.68) formam um sistema não-linear acoplado para as incógnitas $u(y), p$ e $\phi(y)$. Entretanto, essas não-linearidades fazem com que a solução analítica desse sistema seja de difícil obtenção. Para superar essa dificuldade, a seguir, considera-se uma aproximação para o tamanho do ângulo $\phi$.

\subsubsection{Aproximação do Ângulo do Diretor}

Carou et. al.[19, 20, 21] mostraram que na ausência de torques externos (tal como um torque induzido por um campo elétrico), o ângulo de orientação $\phi$ do diretor é pequeno. Logo, na ausência de torques externos, o único torque que atua sobre o ângulo do diretor $\phi$ é devido ao escoamento do fluido. Um escoamento onde $\alpha_{2} \alpha_{3}>0$ é chamado de escoamento alinhado ("flow-aligning materials") e nesses tipos de escoamentos o valor da viscosidade $\alpha_{3}$ é, em geral, duas ordens de magnitude menor que $\alpha_{2}$, ou seja, $\alpha_{3} \ll \alpha_{2}$. Nesta definição, assume-se sempre 
que $\alpha_{2}<0$ e portanto deve-se ter $\alpha_{3}<0$. O ângulo de um escoamento alinhado ou ângulo de Leslie (ver $[82,123]), \phi_{L}$, definido por $\phi_{L}=\tan ^{-1}\left(\alpha_{3} / \alpha_{2}\right)^{\frac{1}{2}}$, é o valor máximo que o escoamento alinhado pode alcançar em um escoamento cisalhante simples. Desde que, $\alpha_{3} \ll \alpha_{2}$ podemos assumir que $\phi_{L} \ll 1$. Em um escoamento alinhado, os efeitos do escoamento tendem a alinhar o diretor de tal forma que $\phi= \pm \phi_{L}$. Logo, o ângulo máximo do diretor no escoamento não será maior que $\phi_{L}$. Portanto, o ângulo $\phi$ do diretor será extremamente pequeno $(\phi \ll 1)$.

Agora considere os seguintes parâmetros: ângulo do escoamento alinhado $\phi_{L}$ e uma escala para o ângulo do diretor, $\beta$, que é definido por $\phi=\beta \phi^{*}$, em que $\phi^{*}=O(1)$. Considerando os parâmetros $\phi_{L}$ e $\beta$, deve-se considerar vários casos dependendo do tamanho do parâmetro $\phi_{L}$.

i) Assumindo que o escoamento tenha apenas um fraco efeito no diretor, então $\beta \ll \phi_{L}$;

ii) Se, entretanto, o escoamento alinha o diretor ao ângulo de Leslie, então $\beta=O\left(\phi_{L}\right)$;

iii) Finalmente, o diretor é alinhado a um ângulo maior que o ângulo de Leslie, assim $\beta \gg \phi_{L}$.

$1^{\circ}$ Caso: $\beta \ll \phi_{L}$ : Os efeitos do escoamento são insuficientes para aumentar $\phi$ significativamente e, desta forma o ângulo de Leslie $\phi_{L}$ não é alcançado. Tem-se que $\beta \ll \phi_{L} \ll 1$, assim, no limite $\beta \rightarrow 0$, as equações (3.66) e (3.68) tornam-se

$$
\begin{aligned}
0 & =-p_{, x}+\frac{1}{R e} u_{, y y}\left(1+\frac{1}{2}\left(\alpha_{3}+\alpha_{6}\right)\right), \\
0 & =\phi_{, y y}-\frac{1}{2} \operatorname{Er}\left(\gamma_{1}+\gamma_{2}\right) u_{, y} .
\end{aligned}
$$

$2^{\circ}$ Caso: $\beta \approx \phi_{L}$ : Os efeitos do escoamento são suficientemente fortes para que o ângulo de Leslie $\phi_{L}$ seja alcançado no escoamento. Logo tomando $\beta=\phi_{L}$ então, no limite $\phi_{L} \rightarrow 0$, as equações (3.66) e (3.68) tornam-se

$$
\begin{aligned}
0 & =-p_{, x}+\frac{1}{R e} u_{, y y}\left(1+\frac{1}{2}\left(\alpha_{3}+\alpha_{6}\right)\right), \\
0 & =\phi_{, y y}-\frac{1}{2} \operatorname{Er}\left(\gamma_{1}+\gamma_{2}\right)\left(1-\frac{\alpha_{2}}{\alpha_{3}} \phi^{2}\right) u_{, y} .
\end{aligned}
$$

$3^{\circ}$ Caso: $\beta \gg \phi_{L}$ : Essa situação não é fisicamente possível se o cristal líquido nemático é do tipo alinhado ("flow-aligning") e nenhum torque externo está presente.

Das equações acima fica claro que as equações para a velocidade $u$ são desacopladas das equações para o ângulo do diretor $\phi$. Portanto, as soluções para o escoamento serão idênticas em cada um dos casos descritos acima e, além disso, serão independentes do ângulo do diretor. Além disso, desde que as equações para o escoamento não envolvam o diretor, a solução para o escoamento será a mesma solução que para um fluido newtoniano e portanto todos os resultados sobre o escoamento podem ser aplicados a fluidos newtonianos. 


\subsubsection{Soluções}

As equações acima (3.70)-(3.71)( $1^{\circ}$ Caso) formam um sistema $2 \times 2$ para as incógnitas $u(y)$ e $\phi(y)$, respectivamente. Para resolver estas equações, as seguintes condições de contorno são utilizadas (ver Figura 3.2.)

$$
\begin{array}{lll}
u=\phi=0 & \text { em } & y=0 \\
u=\phi=0 & \text { em } & y=W
\end{array}
$$

\section{Solução para o perfil de velocidade $u$}

Recordando que a equação para a velocidade $u$ é desacoplada da equação para o ângulo do diretor $\phi$, o perfil da velocidade $u$ pode ser calculado diretamente da equação (3.70). Assim, integrando (3.70) duas vezes e aplicando as condições de contorno acima, obtém-se

$$
u=\frac{R e}{2\left[1+\frac{1}{2}\left(\alpha_{3}+\alpha_{6}\right)\right]} p_{, x} y(y-W)
$$

e portanto,

$$
u_{, y}=\frac{R e}{2\left[1+\frac{1}{2}\left(\alpha_{3}+\alpha_{6}\right)\right]} p_{, x}(2 y-W) .
$$

\section{Solução para o ângulo do diretor $\phi$}

Recordando que, nada pode ser feito em relação ao $3^{\circ}$ Caso, pois é uma situação fisicamente impossível quando se trata de escoamento alinhado ("flow-aligning"), e que pouco progresso analítico pode ser obtido para o $2^{\circ}$ Caso devido a presença do termo $\phi^{2}$ na equação (3.73), nossas atenções serão concentradas no $1^{\circ}$ Caso.

$1^{\circ}$ Caso: $\quad \beta \ll \phi_{L} \ll 1$

Nesse caso, a equação governante é dada pela equação (3.71). Introduzindo o gradiente de velocidade (3.77) na equação de conservação de momento angular (3.71) e integrando-a duas vezes e aplicando as condições de contorno (3.74) e (3.75), encontra-se

$$
\phi(y)=\frac{E r}{2}\left(\gamma_{1}+\gamma_{2}\right) \frac{R e}{2\left[1+\frac{1}{2}\left(\alpha_{3}+\alpha_{6}\right)\right]} p_{, x}\left[\frac{y(W-y)(W-2 y)}{6}\right]
$$

A solução (3.78) mostra que, se $E r=0$ então $\phi=0$; se $E r \neq 0$ então a solução para o ângulo do diretor $\phi$ é uma função cúbica de $y$ diretamente proporcional a Er. Se for necessário calcular a pressão então introduz-se $u(y)$ e $\phi(y)$ na equação (3.67) e integra-se com relação a $y$.

Tendo obtido $\phi$ e $u_{, y}$, as soluções analíticas para as componentes do tensor das tensões não newtoniano $\Phi_{i j}$ são obtidas das equações (3.41)-(3.44), as quais se reduzem à 


$$
\begin{aligned}
\Phi_{x x} & =u_{, y}\left[\left(\alpha_{1}+\alpha_{5}\right) \operatorname{sen} \phi \cos \phi\right] \\
\Phi_{x y} & =\frac{1}{2} u_{, y}\left(\alpha_{3}+\alpha_{6}\right) \\
\Phi_{y x} & =\frac{1}{2} u_{, y}\left(\alpha_{2}+\alpha_{5}\right) \\
\Phi_{y y} & =u_{, y} \alpha_{6} \operatorname{sen} \phi \cos \phi
\end{aligned}
$$

e assim obtém-se a seguinte expressão simplificada para a diferença das tensões normais

$$
\sigma=\Phi_{x x}-\Phi_{y y}=\left(\alpha_{1}+\alpha_{5}-\alpha_{6}\right) \operatorname{sen} \phi \cos \phi u_{, y}
$$

Na equação (3.71), o termo envolvendo $\phi_{\text {,yy }}$ representa os efeitos elásticos enquanto que o termo envolvendo o gradiente de velocidade $u_{, y}$ representa os efeitos do escoamento (hidrodinâmico), devido a viscosidade do cristal líquido nemático. Como dito anteriormente, nesse caso os efeitos do escoamento foram insuficientes para aumentar $\phi$ significativamente de forma que o ângulo de Leslie $\phi_{L}$ não fosse alcançado. Assim os efeitos elásticos afetaram o diretor. Da equação (3.71), nota-se que esse fato é possível somente se o termo

$$
-\frac{1}{2} \operatorname{Er}\left(\gamma_{1}+\gamma_{2}\right) \leq 1
$$

Isto é claro, pois se é desejado assegurar que os efeitos elásticos são no mínimo comparáveis aos efeitos do escoamento, então os termos viscosos não podem dominar os termos elásticos e assim não pode-se ter $-\frac{1}{2} \operatorname{Er}\left(\gamma_{1}+\gamma_{2}\right) \gg 1$. Substituindo os valores adimensionais de $\gamma_{1}$ e $\gamma_{2}$ é possível encontrar que a equação (3.84) é satisfeita para $E r \leq 37.41$.

\subsubsection{Comparação entre as Soluções Numéricas e Analíticas}

Com o objetivo de validar o método numérico apresentado nesse Capítulo, o escoamento de um cristal líquido nemático MBBA à $25 C^{\circ}$ em um canal bidimensional (como descrito na Figura 3.2) foi simulado e as soluções numéricas foram comparadas com as respectivas soluções analíticas. As condições de contorno para o campo de velocidade foram especificadas na Seção 3.2.1 . No contorno de entrada de fluido foi imposto um escoamento newtoniano completamente desenvolvido dado por

$$
U_{\text {inf }}=u(y)=-4 \frac{U}{W^{2}}\left(y-\frac{W}{2}\right)^{2}+U
$$

Para simular este problema, os seguintes dados foram empregados:

- Distância entre as placas: $W=0.001 \mathrm{~m}=1 \mathrm{~mm}$;

- Comprimento do canal: $L=20 W=0.02 \mathrm{~m}=20 \mathrm{~mm}$; 
- Velocidade: $U=0.0005 \mathrm{~ms}^{-1}=0.5 \mathrm{mms}^{-1}$;

- Viscosidade: $\eta=\frac{1}{2} \alpha_{4}=0.0413$ Pa s;

- Densidade: $\rho=1088 \mathrm{Kg} / \mathrm{m}^{3}$

Os parâmetros físicos, especificando o cristal líquido nemático $\mathrm{MBBA}$ à $25 \mathrm{C}^{\circ}$ são dados nas Tabelas A.1 e A.2 no apêndice A. Com estes dados obtém-se $R e=0.0132$ e $E r=10$.

Para demonstrar a convergência do método numérico apresentado nesse trabalho, simulamos o escoamento em um canal bidimensional utilizando três malhas:

- $\mathrm{M}_{0}: \delta x=\delta y=0.000125 \mathrm{~m}(160 \times 8$ células $)$

- $\mathrm{M}_{1}: \delta x=\delta y=0.0000625 \mathrm{~m}(320 \times 16$ células $)$

- $\mathrm{M}_{2}: \delta x=\delta y=0.00003125 \mathrm{~m}(640 \times 32$ células $)$

Com esses dados, o escoamento no canal bidimensional foi simulado até $L / U t=80$ s (tempo adimensional $t=160$ ). Espera-se que no tempo $t=80 \mathrm{~s}$ o estado estacionário tenha sido alcançado e que as soluções numéricas aproximem as respectivas soluções analíticas.

Para demonstrar que as soluções estacionárias foram alcançadas, a Figura 3.3 mostra as isolinhas de $u, v$ e $p$ em $t=80$ s. Pode-se observar na Figura 3.3 que a velocidade $v$ é nula em todo o domínio e que as isolinhas da velocidade $u$ estão paralelas e variam somente com $y$ enquanto o gradiente de pressão varia com $x$. Para confirmar, quantitativamente, que as condições de escoamento totalmente desenvolvido foram encontradas, a variação da pressão e do ângulo do diretor na linha central do canal são mostrados na Figura 3.4, enquanto as velocidades $u$ e $v$, na seção do canal em $x=10$, são mostradas na Figura 3.5. Pode-se observar nas Figuras 3.4 e 3.5 que $p, \phi, u$ e $v$ que, de fato, as condições de escoamento totalmente desenvolvido foram alcançadas em $t=80 \mathrm{~s}$. Portanto, pode-se concluir que em $t=80 \mathrm{~s}, \mathrm{o}$ estado estacionário foi atingido.

Para avaliar as soluções analíticas por meio das equações (3.76), (3.78), e (3.79)- (3.82), o valor do gradiente de pressão precisa ser conhecido. Um valor apropriado de $\frac{\partial p}{\partial x}$ pode ser determinado tomando a componente $u$ da velocidade (3.76) igual a velocidade correspondente da entrada do canal dada pela equação (3.85). Seguindo este procedimento e utilizando os dados de entrada descritos acima, encontrou-se $p_{, x}=-351.4272$.

Para mostrar que as soluções numéricas aproximam as soluções analíticas (ver equações (3.76), (3.78), e (3.79)- (3.82)), as Figuras 3.7, 3.8, 3.9 e 3.10 mostram as soluções numéricas obtidas no meio do canal $(x=10 \mathrm{~W})$ juntamente com as soluções analíticas. Pode-se observar nas Figuras 3.7, 3.8, 3.9 e 3.10 que há uma boa concordância entre as soluções numéricas e as soluções analíticas nas três malhas utilizadas. Além disso, os resultados numéricos mostram que quando a malha é refinada as soluções numéricas convergem para as respectivas soluções analíticas. 

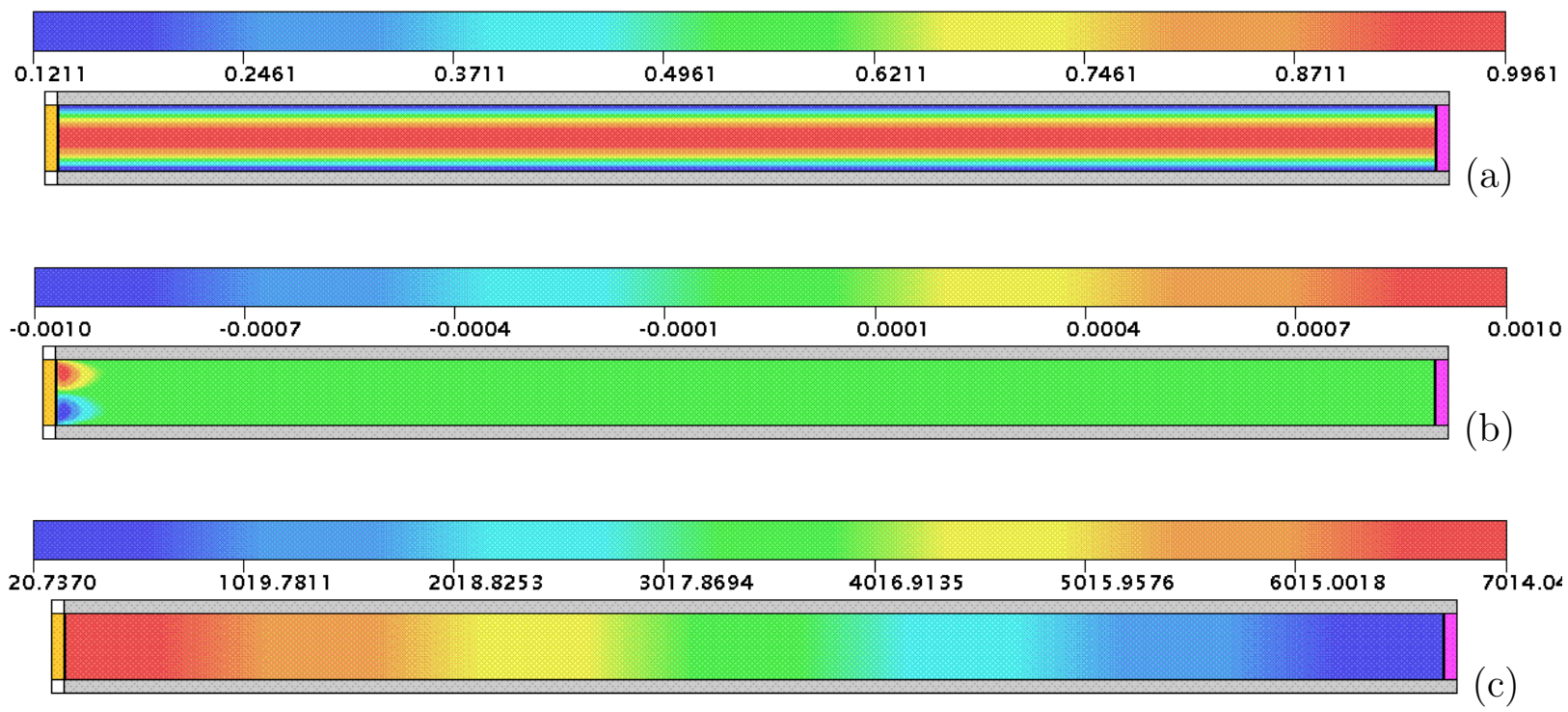

Figura 3.3: Isolinhas de: (a) $u$, (b) $v$ e (c) $p$ em $t=80$ s.

Para demonstrar quantitativamente que a convergência é obtida, o erro relativo da solução numérica $\left(\operatorname{Sol}_{N U M}\right)$ foi calculado nas três malhas através da fórmula

$$
E\left(\operatorname{Sol}_{N U M}\right)=\sqrt{\frac{\sum_{i j}\left(\operatorname{Sol}_{E X A T A}-\mathrm{Sol}_{N U M}\right)^{2}}{\sum_{i j}\left(\operatorname{Sol}_{E X A T A}\right)^{2}}} .
$$

em que $\operatorname{Sol}_{E X A T A}$ representa a solução definida pelas equações (3.76), (3.78), e (3.79)- (3.82). Os valores dos erros relativos obtidos nas três malhas $\mathrm{M}_{0}, \mathrm{M}_{1}$ e $\mathrm{M}_{2}$ são mostrados na Tabela 3.1 e a Figura 3.6 mostra o decaimento dos erros relativos com o refinamento da malha. Pode-se observar na Tabela 3.1 que os erros decrescem à medida que a malha é refinada; esses resultados mostram que o método numérico é de fato convergente. Além disso, utilizando os resultados da Tabela 3.1, uma estimativa da ordem de convergência do método numérico foi calculada pela fórmula

$$
N_{i}(*)=\frac{\log \left[\frac{E(*) M_{i+1}}{E(*) M_{i}}\right]}{\log \left[\frac{\delta x M_{i+1}}{\delta x M_{i}}\right]}, i=0,1 .
$$

Para a componente $u$ da velocidade encontramos $N_{0}(u)=2.32$ e $N_{1}(u)=1.48$. Estes resultados mostram que o método numérico apresentado nesse trabalho é convergente. Mais ainda, esses resultados validam o método numérico (e o software associado) para resolver escoamentos bidimensionais de cristais líquidos nemáticos sob efeito de campo magnético. 

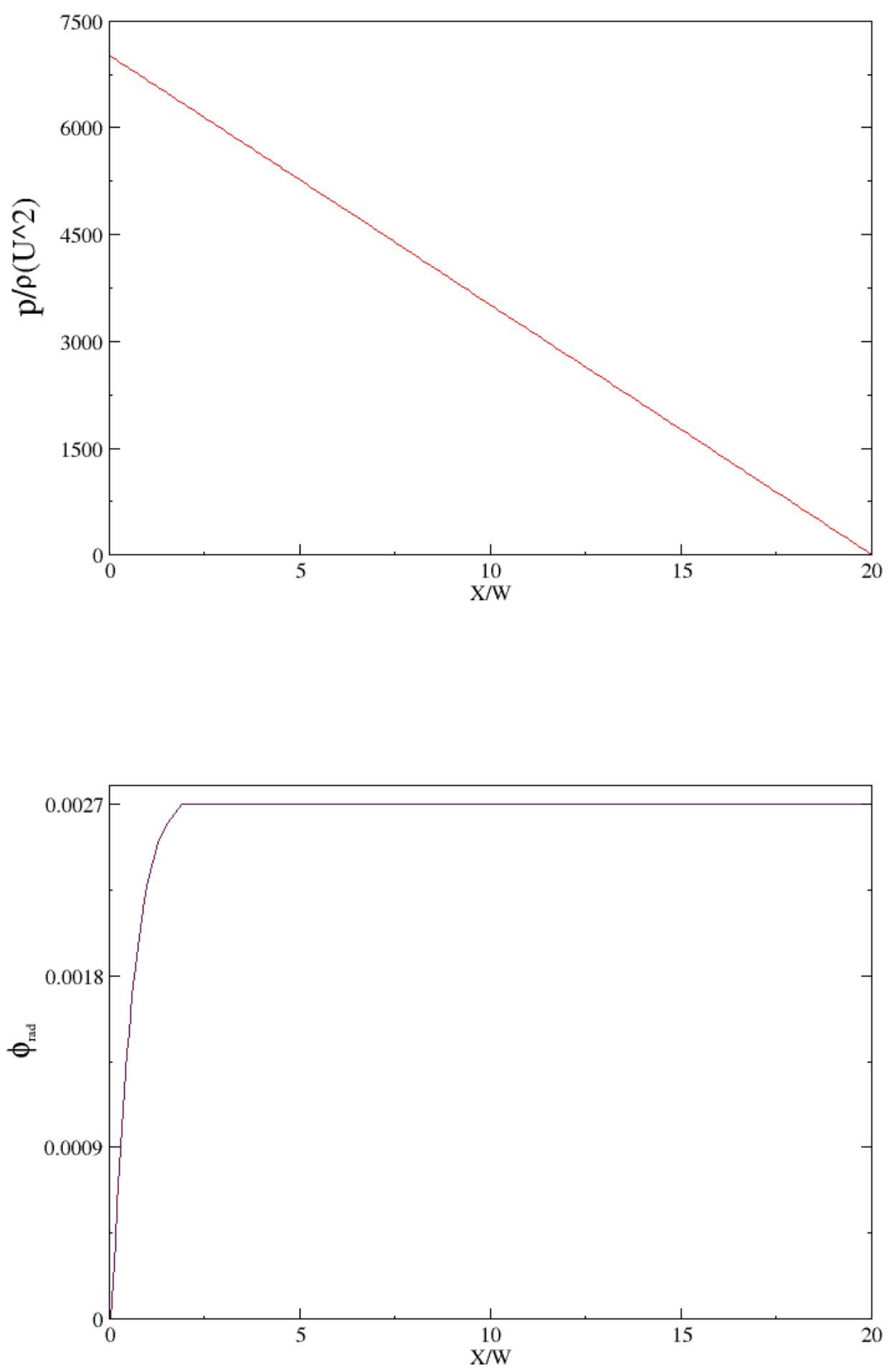

Figura 3.4: Simulação numérica do escoamento de um cristal líquido nemático em um canal. Variação de $p(x, y c)$ e $\phi\left(x, y_{c}\right), 0 \leq x \leq 20, y_{c}=0.5+0.5 \delta y$, no tempo $t=80$ s. Note que $x$ e $y$ foram adimensionalizadas pela largura do canal $W$. 


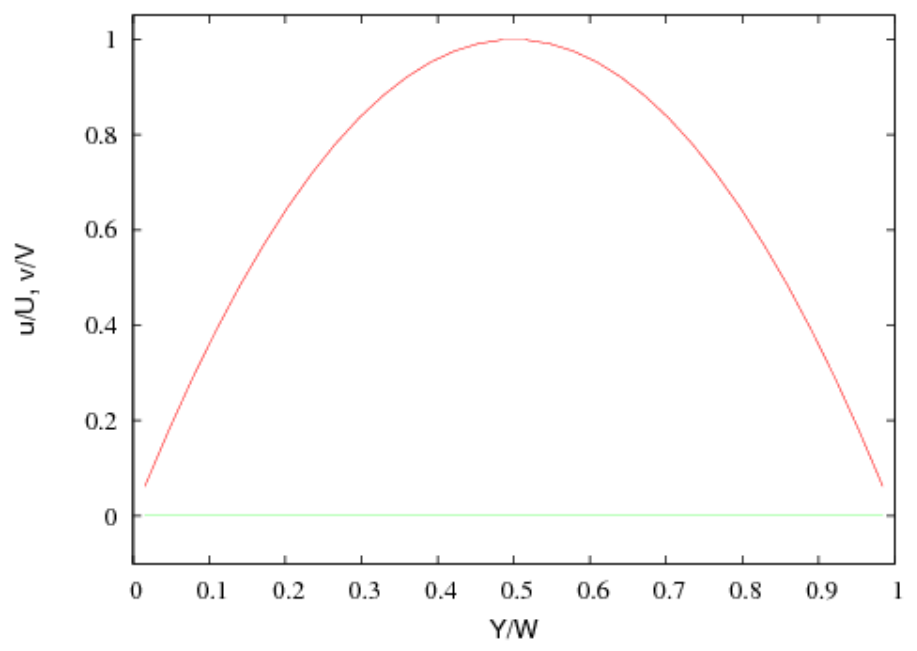

Figura 3.5: Simulação numérica do escoamento de um cristal líquido nemático em um canal. Variação de $u\left(x_{c}, y\right)$ e $v\left(x_{c}, y\right), 0 \leq y \leq 1, x_{c}=10+0.5 \delta x$, no tempo $t=80$ s. Note que $x$ e $y$ foram adimensionalizadas pela largura do canal $W$.

Tabela 3.1: Erros Relativos obtido nas malhas $\mathrm{M}_{0}, \mathrm{M}_{1}$ e $\mathrm{M}_{2}$.

\begin{tabular}{|c||c|c|c||c|c|}
\hline \multirow{2}{*}{ Variáveis } & \multirow{2}{*}{$\begin{array}{c}\text { Erro Relativo } \\
\mathrm{M}_{0}\end{array}$} & $\begin{array}{c}\text { Erro Relativo } \\
\mathrm{M}_{1}\end{array}$ & $\begin{array}{c}\text { Erro Relativo } \\
\mathrm{M}_{2}\end{array}$ & \multicolumn{2}{|c|}{ Ordem de Convergência } \\
\cline { 5 - 6 } & $N_{0}$ & $N_{1}$ \\
\hline \hline$U$ & $1.36752 \times 10^{-2}$ & $2.73927 \times 10^{-3}$ & $9.84150 \times 10^{-4}$ & 2.32 & 1.48 \\
\hline$\Phi_{x x}$ & $6.03090 \times 10^{-2}$ & $1.95730 \times 10^{-2}$ & $8.18080 \times 10^{-3}$ & 1.62 & 1.26 \\
\hline$\Phi_{x y}$ & $2.40981 \times 10^{-2}$ & $6.81500 \times 10^{-3}$ & $2.58160 \times 10^{-3}$ & 1.82 & 1.40 \\
\hline$\Phi_{y x}$ & $2.29802 \times 10^{-2}$ & $5.80770 \times 10^{-3}$ & $1.58004 \times 10^{-3}$ & 1.98 & 1.88 \\
\hline$\Phi_{y y}$ & $5.89102 \times 10^{-2}$ & $1.77806 \times 10^{-2}$ & $7.28004 \times 10^{-3}$ & 1.72 & 1.29 \\
\hline$\phi$ & $5.7260 \times 10^{-2}$ & $1.53670 \times 10^{-2}$ & $9.8370 \times 10^{-3}$ & 1.90 & 0.64 \\
\hline
\end{tabular}

A Figura 3.11 mostra o gráfico para diferentes números de Ericksen para a solução dada pela equação (3.78). Pode-se observar na Figura 3.11 que quando $E r=0$ temos a solução nula e, quando $E r \neq 0$ tem-se que a solução para o ângulo do diretor $\phi$ é uma função cúbica de $y$ diretamente proporcional ao número de Ericksen.

A Figura 3.12 apresenta a comparação da solução numérica com a solução analítica para o ângulo do diretor $\phi$. Pode-se observar que para $\operatorname{Er}=20$ existe um boa concordância entre a solução obtida numericamente e a solução analítica, enquanto que para $E r=35$ essa boa concordância deixa de existir, pois a solução dada pela equação (3.78) é válida apenas quando os efeitos do escoamento são menores que os efeitos elásticos. 
(a)

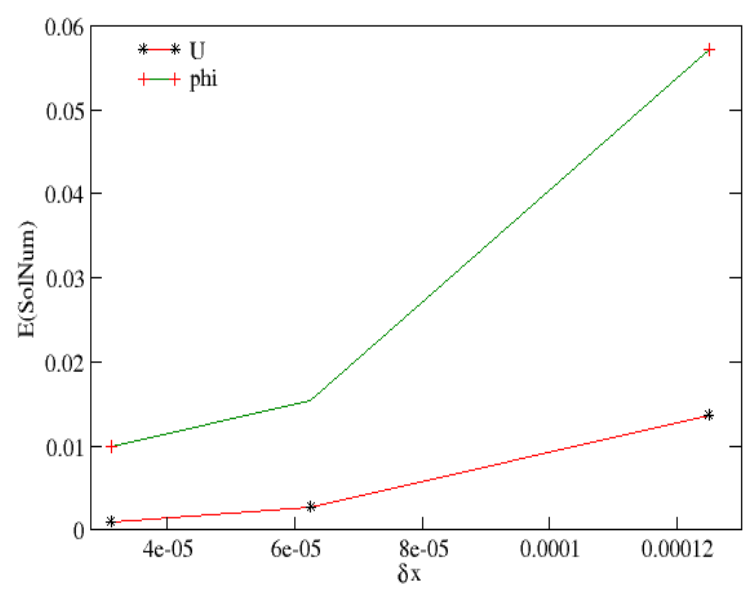

(b)

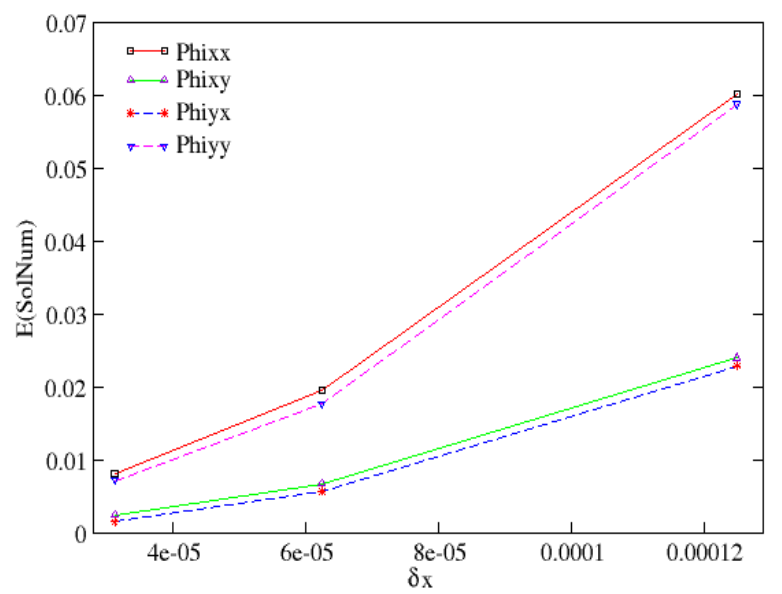

Figura 3.6: Decaimento dos erros relativos com o refinamento da malha: (a) componente $u$ da velocidade e ângulo do diretor $\phi$ e (b) componentes do tensor não-newtoniano $\Phi_{x x}, \Phi_{x y}, \Phi_{y x}$, e $\Phi_{y y}$.

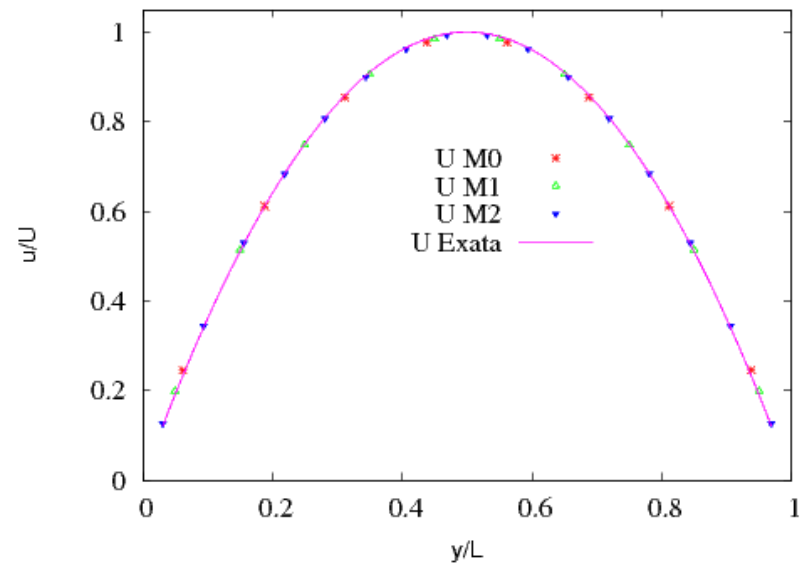

Figura 3.7: Solução numérica e analítica da componente $u\left(x_{c}, y\right), x_{c}=10$, da velocidade para $R e=0.0132$ e $E r=10$. 

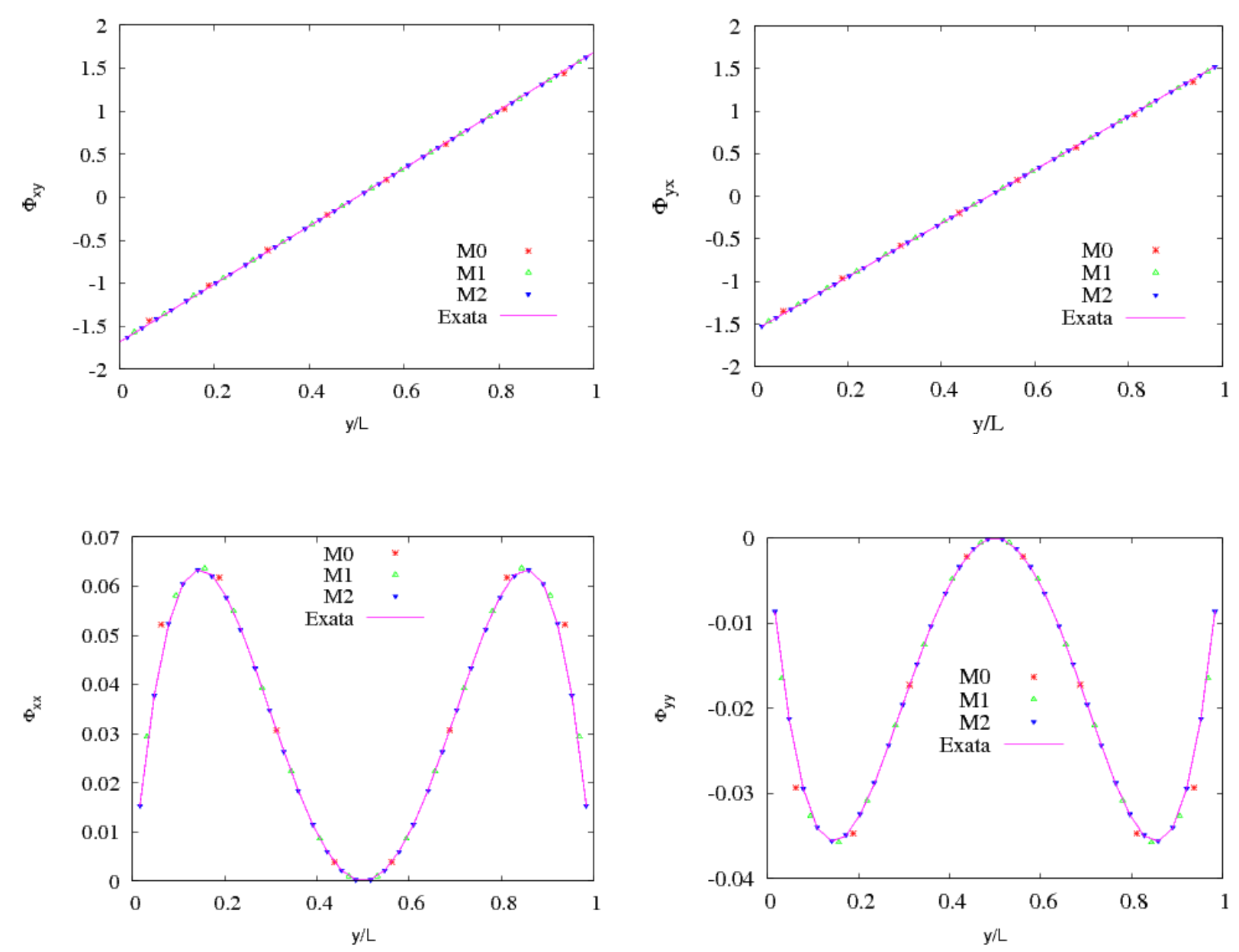

Figura 3.8: Comparação entre os resultados numéricos e analítico das componentes do tensor das tensões não-newtoniano $\Phi_{x y}\left(x_{c}, y\right), \Phi_{y x}\left(x_{c}, y\right), \Phi_{x x}\left(x_{c}, y\right)$ e $\Phi_{y y}\left(x_{c}, y\right), x_{c}=10$. 


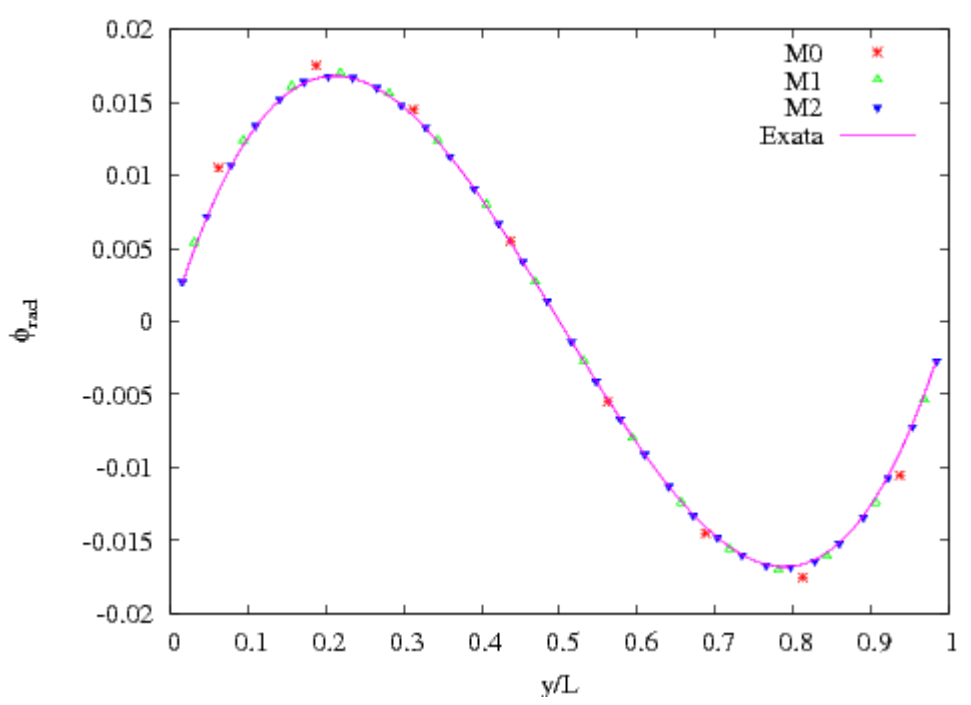

Figura 3.9: Comparação das soluções numérica e analítica do ângulo do diretor $\phi\left(x_{c}, y\right), x_{c}=10$ para $R e=0.0132$ e $E r=10$ nas malhas $\mathrm{M}_{0}, \mathrm{M}_{1}, \mathrm{M}_{2}$.
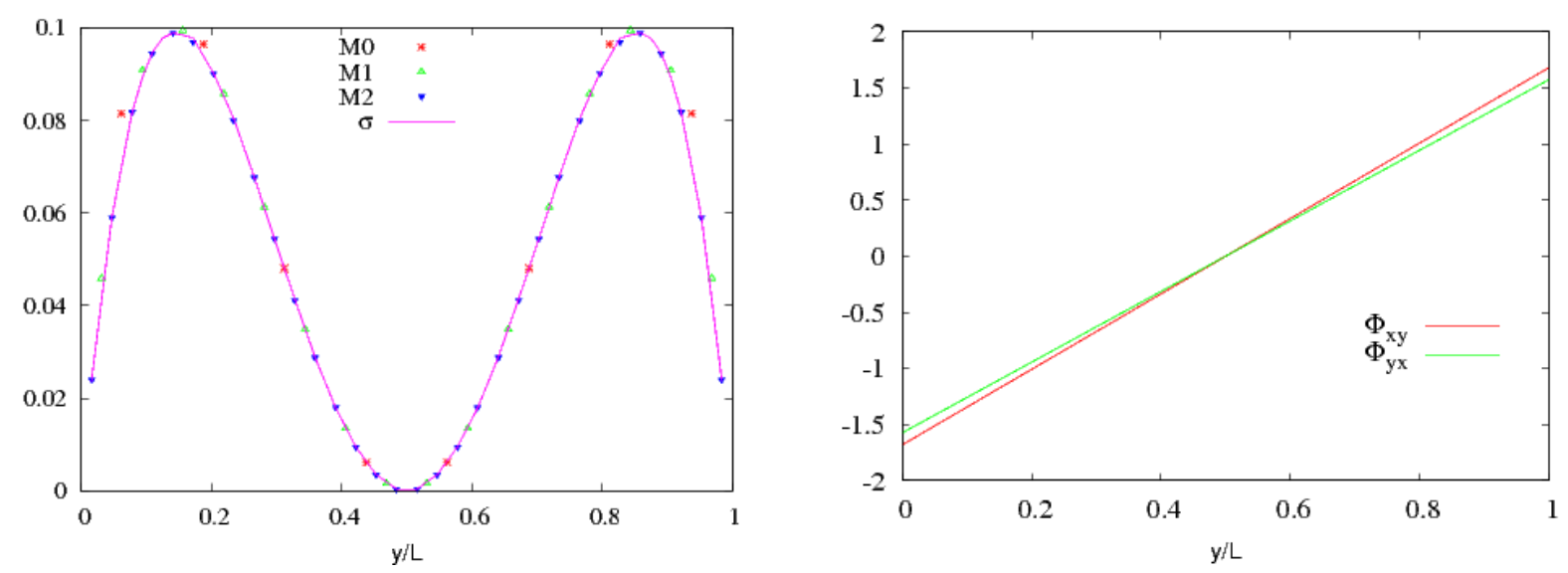

Figura 3.10: Comparação das soluções numéricas e analítica para a primeira diferença de tensões $\sigma=u_{, y}\left(\alpha_{1}+\alpha_{5}-\alpha_{6}\right) \operatorname{sen} \phi \cos \phi$ (à esquerda) e comparação dos tensores $\Phi_{x y}$ e $\Phi_{y x}$ (à direita). 

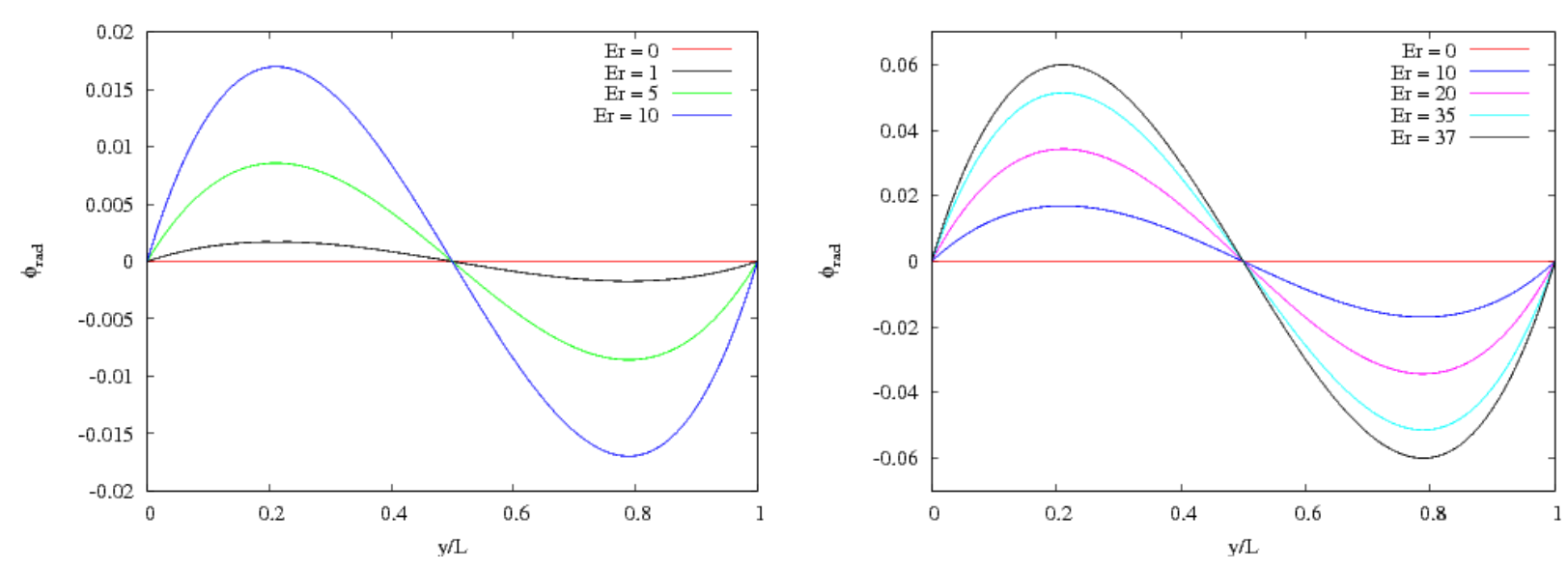

Figura 3.11: Gráfico da soluções analíticas para a equação (3.78) obtida na Seção 3.5.1.2 para vários números de Ericksen.
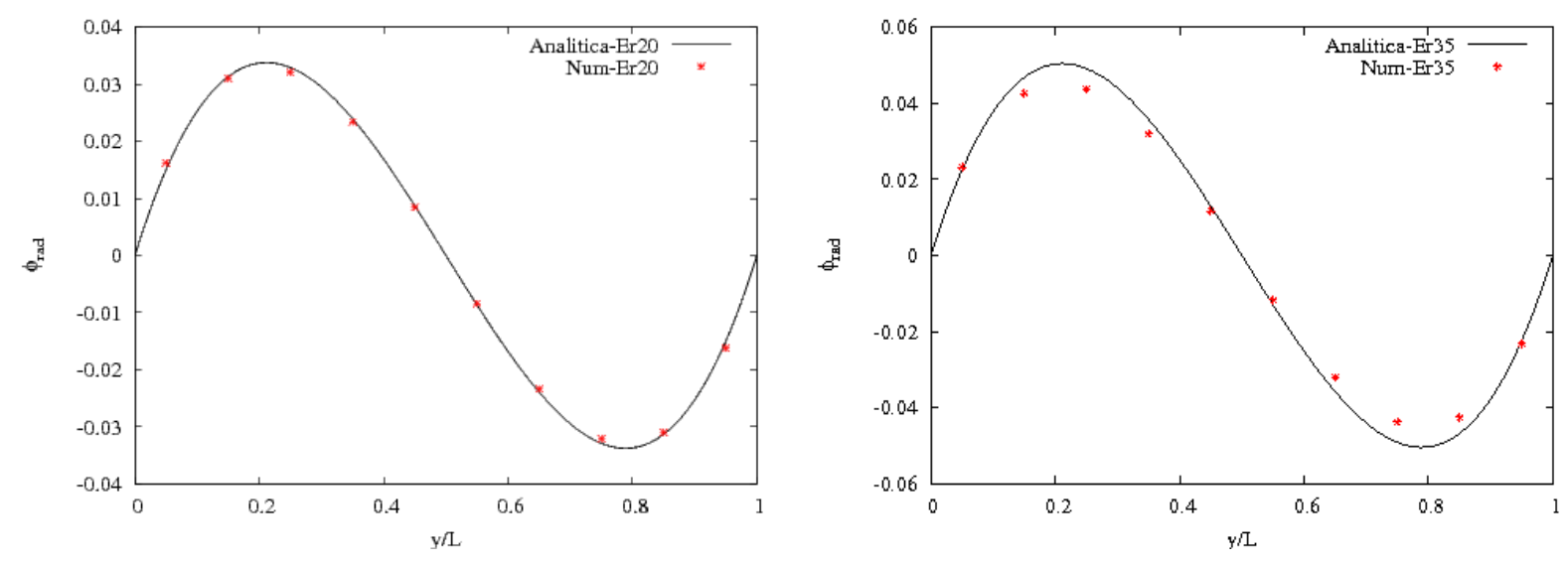

Figura 3.12: Comparação das soluções numéricas obtidas na saída do canal com a solução analítica dada pela equação (3.78) para: $E r=20$ e $E r=35$. 


\section{Simulação Numérica de Escoamentos}

\section{Bidimensionais de Cristais Líquidos Nemáticos}

Neste Capítulo, a técnica numérica apresentada no Capítulo 3 é utilizada para simular escoamentos bidimensionais de cristais líquidos nemáticos sob efeito de um campo magnético. Os escoamentos de um cristal líquido nemático em geometrias complexas, tais como L-canal (canal em forma de L), contração planar 4:1 e expansão planar 4:1, são considerados.

\subsection{Investigação Numérica do Escoamento em L-canais}

Nessa seção considera-se o escoamento de cristal líquido nemático em um L-canal como mostrado na Figura 4.1. Nas paredes do canal tomou-se $u_{i}=0$ enquanto que na entrada do canal a velocidade foi dada pela equação (3.85) apresentada na Seção 3.5.2. Na saída do canal a velocidade obedeceu a condição homogênea de Neumann $u_{, y}=v_{, y}=0$.

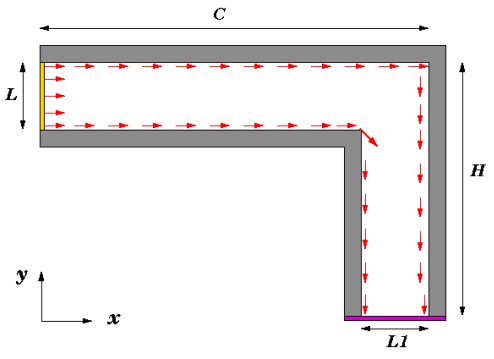

(a)

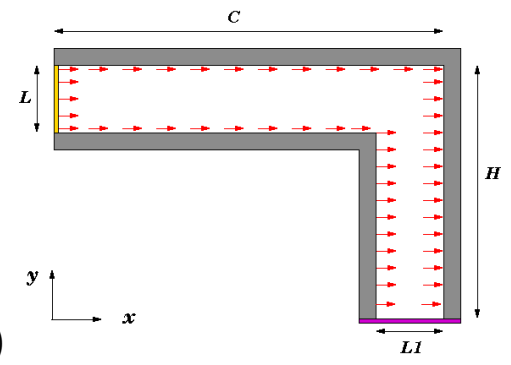

(b)

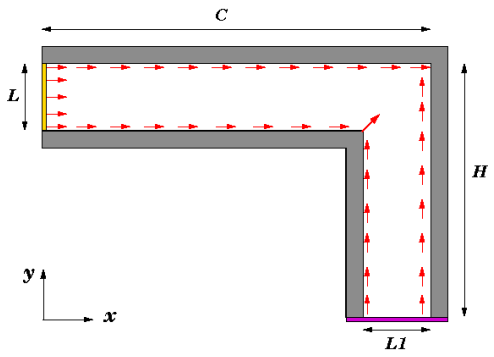

(c)

Figura 4.1: Definição do domínio de simulação do escoamento em um L-canal. As setas vermelhas representam as condições de contorno utilizadas para o cálculo do ângulo $\phi$ por meio da equação (3.36). 


\subsubsection{Efeito das condições de contorno para o ângulo $\phi$}

Pretende-se estudar o efeito das condições de contorno no escoamento e para isso, as seguintes condições de contorno para o ângulo $\phi$ foram especificadas:

1. Ao longo das paredes horizontais, o ângulo de ancoragem foi tomado como nulo, implicando em alinhamento paralelo nas paredes.

2. Na quina formada na mudança de orientação do canal, utilizou-se:
(a) $\phi=-45^{\circ}$ (veja Figura 4.1(a));
(b) $\phi=0$ (veja Figura 4.1(b));
(c) $\phi=45^{\circ}($ veja Figura 4.1(c)).

3. Nas paredes verticais, adotou-se as seguintes condições:

(a) $\phi=-90^{\circ}$ em $x=C, 0 \leq y \leq H$ e $x=C-L 1,0 \leq y \leq H-L$ (veja Figura 4.1(a));

(b) $\phi=0$ em $x=C, 0 \leq y \leq H$ e $x=C-L 1,0 \leq y \leq H-L$ (veja Figura 4.1(b));

(c) $\phi=90^{\circ}$ em $x=C, 0 \leq y \leq H$ e $x=C-L 1,0 \leq y \leq H-L$ (veja Figura 4.1(c)).

4. Na entrada do canal tomou-se $\phi=0$ e na saída foi especificado $\phi_{, y}=0$.

Os parâmetros físicos referentes ao cristal líquido nemático MBBA a $25 C^{\circ}$ empregados para simular este problema são apresentados nas Tabelas A.1, A.2 e A.3 do apêndice A.

Os outros parâmetros utilizados foram:

- Malha: $60 \times 60$ células $(\delta x=\delta y=0.0001 \mathrm{~m})$;

- Largura da entrada do canal: $L=0.001 \mathrm{~m}$;

- Comprimento horizontal do canal: $C=0.006 \mathrm{~m}$;

- Comprimento vertical do canal: $H=0.006 \mathrm{~m}$;

- Velocidade na entrada do canal: $V_{\text {inf }}=0.001 \mathrm{~ms}^{-1}$;

- Velocidade de escala: $U=0.001 \mathrm{~ms}^{-1}$;

Nestas simulações, variou-se a largura da saída do canal $(L 1)$ e esse problema foi simulado para as seguintes razões $(R i=L 1 / L): R 1=1, R 2=0.4$ e $R 3=0.3$. Os parâmetros de escala utilizados foram a velocidade $U$, a largura da entrada do canal $L$ e a viscosidade $\eta$, e assim obteve-se $R e=0.026$. Nos resultados a seguir os seguintes números de Ericksen $\left(E r=\frac{U L \eta}{K}\right)$ foram empregados: 


\begin{tabular}{|c|c|c|c|}
\hline Número de Ericksen & \multicolumn{3}{|c|}{ Constantes Elásticas } \\
\hline Er & $R 1=1$ & $R 2=0.4$ & $R 3=0.3$ \\
\hline 5 & $K=8.26 \times 10^{-9}$ & - & - \\
\hline 10 & - & $K=4.13 \times 10^{-9}$ & $K=4.13 \times 10^{-9}$ \\
\hline 50 & $-\quad-$ & $K=8.26 \times 10^{-10}$ & $K=8.26 \times 10^{-10}$ \\
\hline 100 & - & $K=4.13 \times 10^{-10}$ & $K=4.13 \times 10^{-10}$ \\
\hline 150 & - & $K=2.753 \times 10^{-10}$ & $K=2.753 \times 10^{-10}$ \\
\hline 200 & - & $K=2.065 \times 10^{-10}$ & $K=2.065 \times 10^{-10}$ \\
\hline 250 & - & $K=1.652 \times 10^{-10}$ & $K=1.652 \times 10^{-10}$ \\
\hline
\end{tabular}

Para demonstrar que as condições de contorno aplicadas para o cálculo do ângulo $\phi$ podem afetar o campo dos diretores, realizou-se três simulações com $R e=0.026$ e $E r=5$. Na primeira simulação utilizou-se as condições de contorno apresentadas na Figura 4.1(a) e na segunda e terceira simulações empregamos as condições apresentadas na Figura 4.1(b) e Figura 4.1(c), respectivamente. Essas simulações foram realizadas até o estado estacionário ser alcançado.

A Figura 4.2 mostra as isolinhas da pressão e das velocidades $u$ e $v$ obtidas na simulação utilizando as condições de contorno para o diretor apresentadas na Figura 4.1(a). Na Figura $4.2(\mathrm{a})$, as isolinhas na entrada do canal indicam que a pressão varia somente com $x$ e as isolinhas na saída do canal mostram que a pressão varia somente com $y$. Isso é confirmado nas Figuras 4.3(a) e 4.3(b) que mostram a variação da pressão nos respectivos canais.

Por outro lado, as isolinhas na Figura 4.2(b) mostram que a velocidade $u$ é zero à jusante do canal e são paralelas a montante do canal. Um resultado semelhante foi obtido para a velocidade $v$ conforme é mostrado na Figura 4.2(c). Esses fatos são confirmados nas Figuras 4.4(a) e 4.4(b) onde pode-se observar que ambas as velocidades $u$ e $v$ possuem o mesmo perfil parabólico nos respectivos canais. Esses resultados mostram que o estado estacionário foi atingido. Resultados semelhantes para os perfis de $u$ e $v$ foram obtidos quando as condições de contorno especificadas nas Figuras 4.1(b) e 4.1(c) foram utilizadas.

Um zoom das soluções obtidas para os diretores, próximo a quina formada pela mudança de direção canal, é mostrado na Figura 4.5. Pode-se observar na Figura 4.5 que as condições de contorno aplicadas no ângulo $\phi$ produziram um efeito no campo dos diretores. Na primeira simulação (veja a Figura 4.5(a)), o perfil do diretor não mostrou grandes variações, o ângulo de orientação do diretor é quase uniforme no L-canal e concorda com as condições de contorno impostas. Na segunda simulação (veja a Figura 4.5(b)), o perfil do diretor, à jusante do canal, foi fortemente afetado pelas condições de contorno que forçaram o ângulo do diretor tornar-se $0^{\circ}$ ao longo das paredes à jusante do canal. De forma similar, na terceira simulação, quando o ângulo de orientação $\phi$ ao longo das paredes à jusante do canal foi tomado no sentido oposto a direção do escoamento principal $\left(90^{\circ}\right)$, o ângulo do diretor foi novamente afetado fortemente pelas condições de contorno que fizeram o diretor ficar a $90^{\circ}$ ao longo das paredes a jusante do canal. As Figuras 4.6, 4.7 e 4.8 mostram a variação do ângulo do diretor no centro do L-canal 
com as condições de contorno apresentadas nas Figuras 4.1(a), 4.1(b) e 4.1(c). Pode-se observar nessas figuras que as condições de contorno afetaram o campo dos diretores que por sua vez adquiriram valores de acordo com as condições de contorno impostas.

A Figura 4.9 mostra que as várias condições de contorno aplicadas ao ângulo do diretor não produziram maiores mudanças nas linhas de corrente da velocidade no L-canal: as linhas de corrente são suaves ao longo do L-canal. Tal comportamento foi semelhante aos resultados observados por Chono et al. [24] para certos fluidos viscoelásticos.

(a)

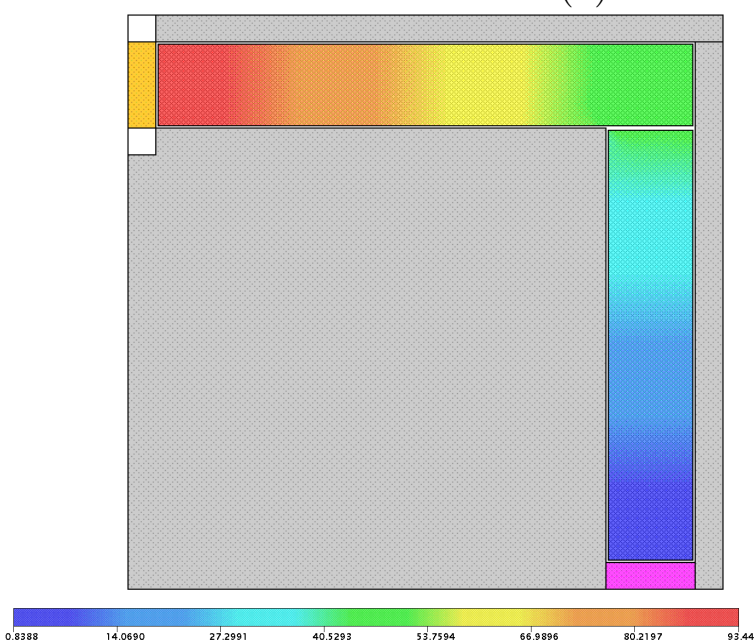

(b)

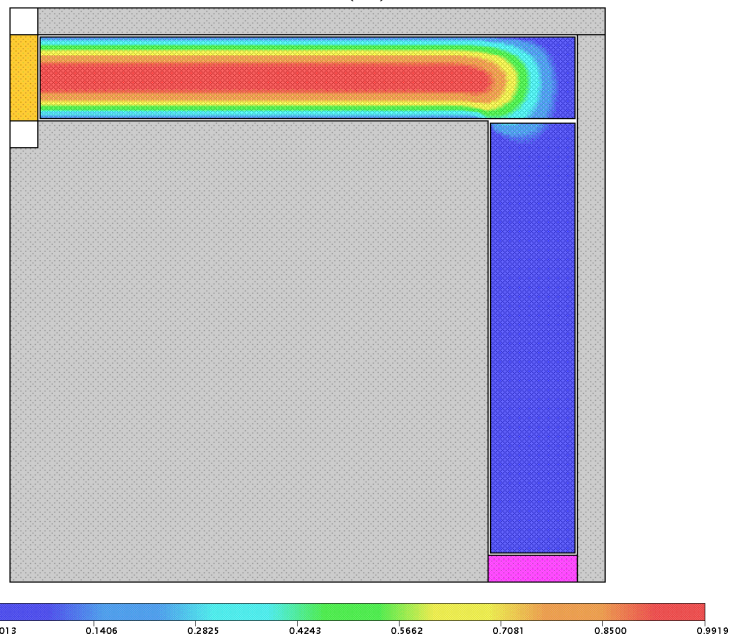

(c)

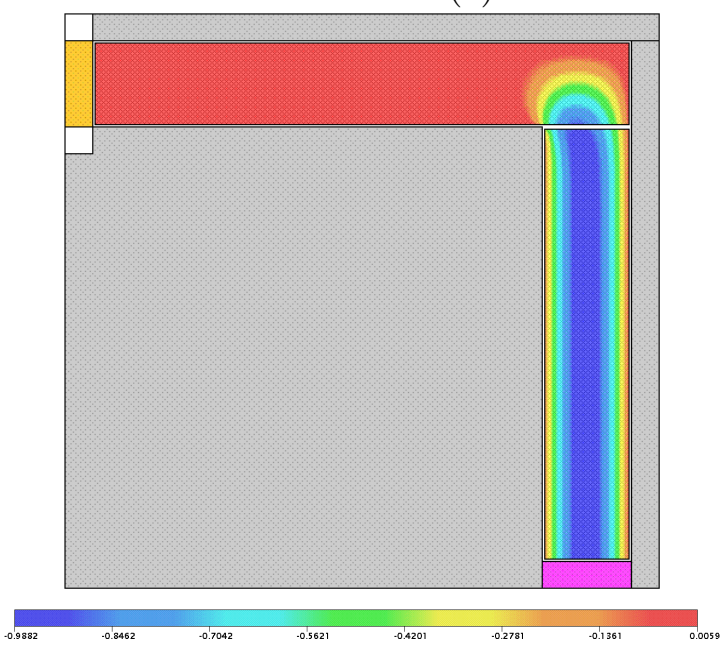

Figura 4.2: Simulação Numérica do escoamento através de um L-canal com $R e=0.026$ e $E r=5$. Isolinhas: (a) Pressão, (b) velocidade $u$ e (c) velocidade $v$. 


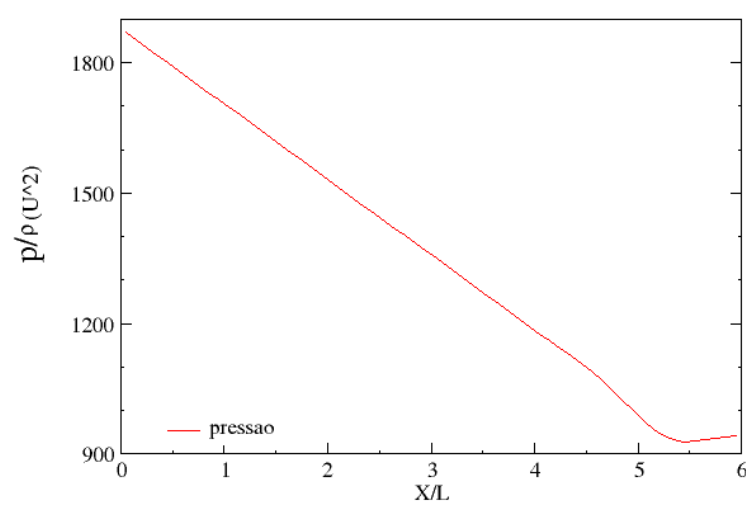

(a)

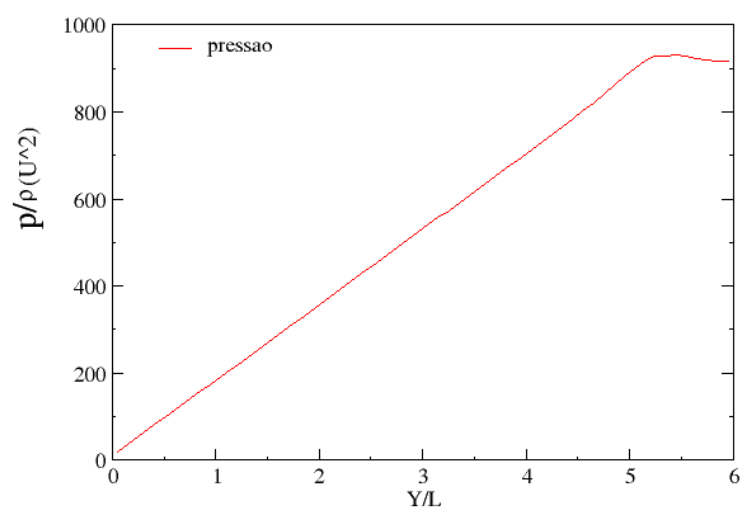

Figura 4.3: Variação da pressão no L-canal com $R e=0.026$ e $E r=5$ e condição de contorno $\phi=-90^{\circ}$. (a) Pressão no canal de entrada para $y=5.55$ e $0 \leq x \leq 6$. (b) Pressão no canal de saída para $x=5.55$ e $0 \leq y \leq 6$.

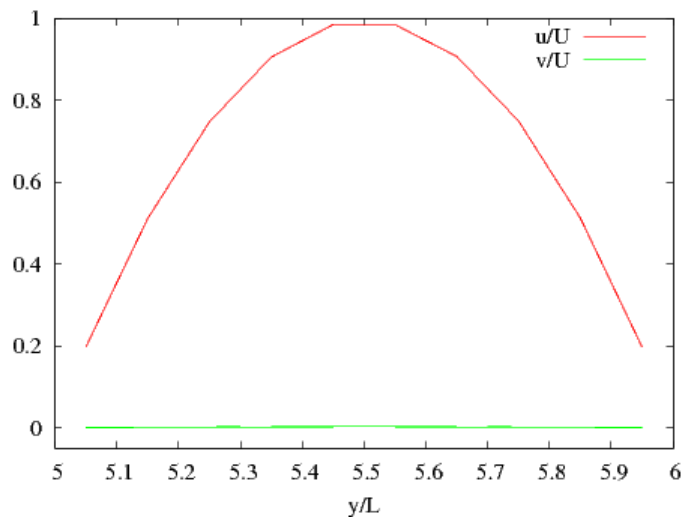

(a)

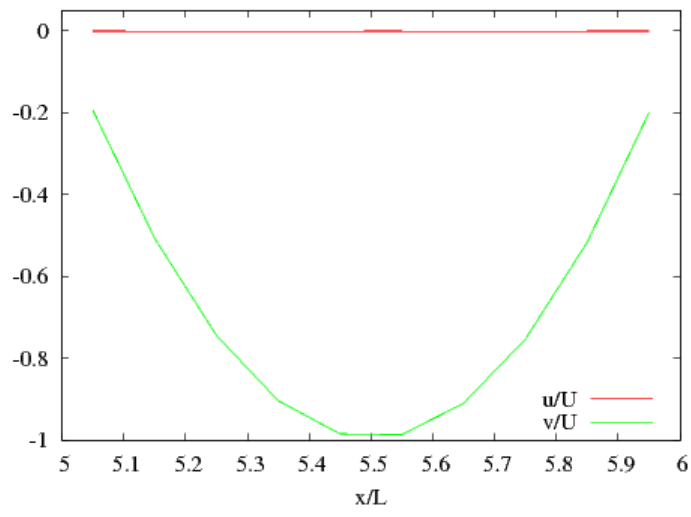

(b)

Figura 4.4: Variação das velocidades no L-canal com $R e=0.026$ e $E r=5$ e condição de contorno $\phi=-90^{\circ}$. (a) Velocidade no canal de entrada para $x=4.55$ e $5 \leq y \leq 6$. (b) Velocidade no canal de saída para $y=4.55$ e $5 \leq x \leq 6$. 

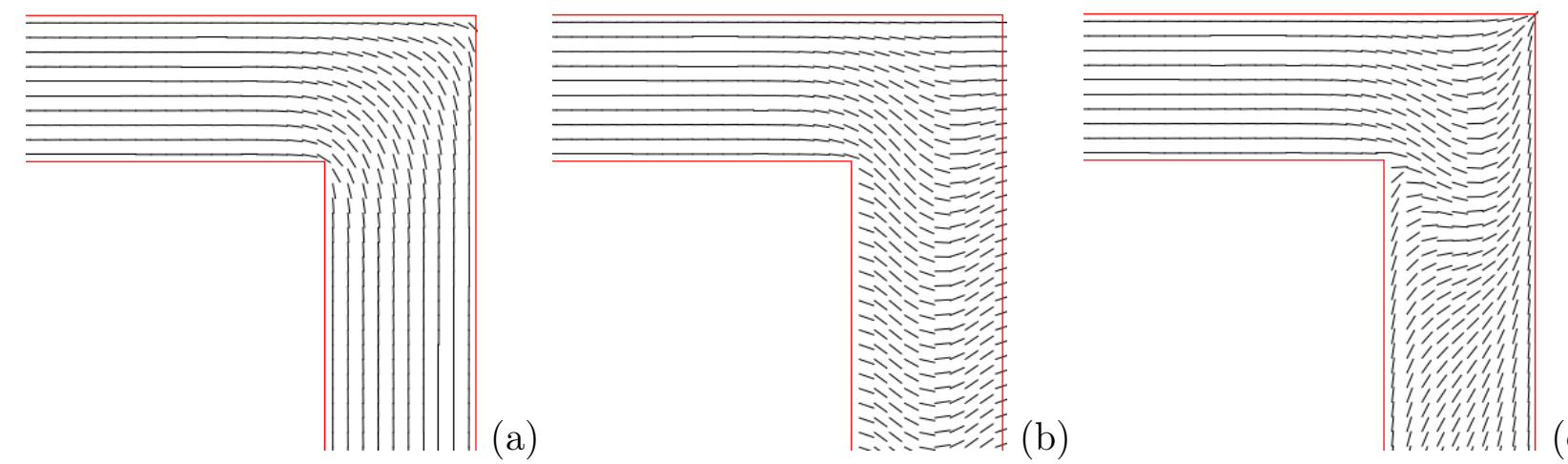

(c)

Figura 4.5: Simulação numérica do diretor através de um L-canal com $R e=0.026$ e $E r=5$. Distribuição do diretor para diferentes condições de contorno para o ângulo $\phi$ : (a) $\phi=-90^{\circ}$, (b) $\phi=0$, e (c) $\phi=90^{\circ}$.

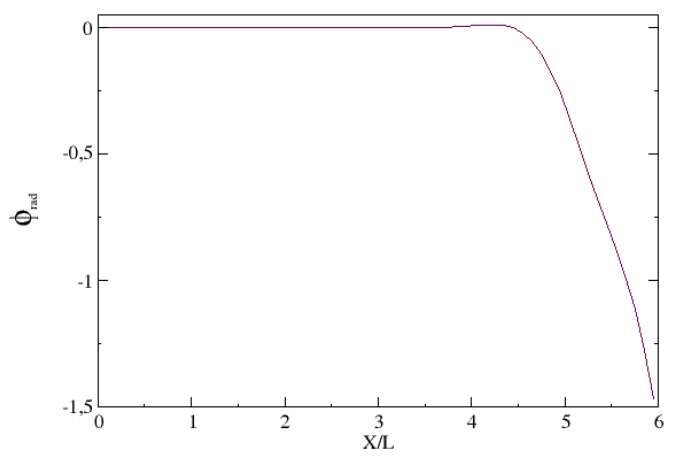

(a)

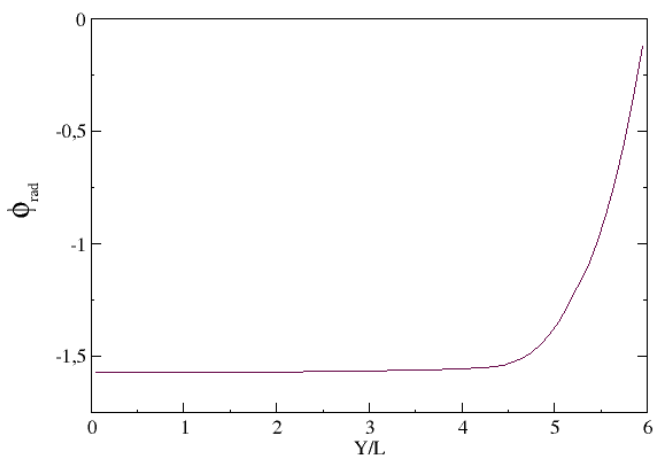

(b)

Figura 4.6: Variação do ângulo do diretor no L-canal com $R e=0.026$ e $E r=5$ e condição de contorno $\phi=-90^{\circ}$. (a) $y=5.55$ e $0 \leq x \leq 6$; (b) $x=5.55$ e $0 \leq y \leq 6$.

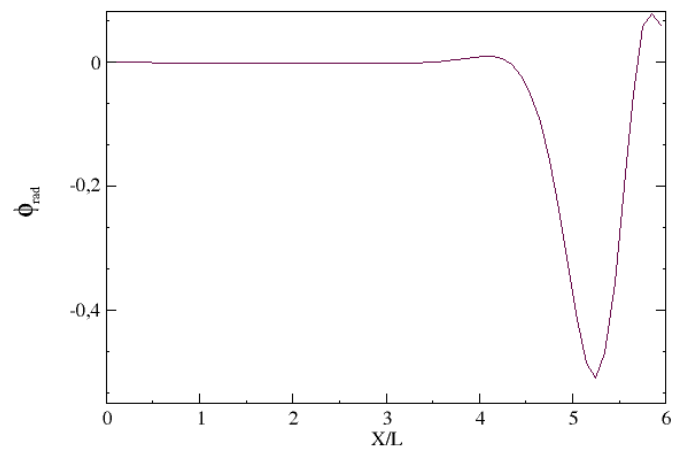

(a)

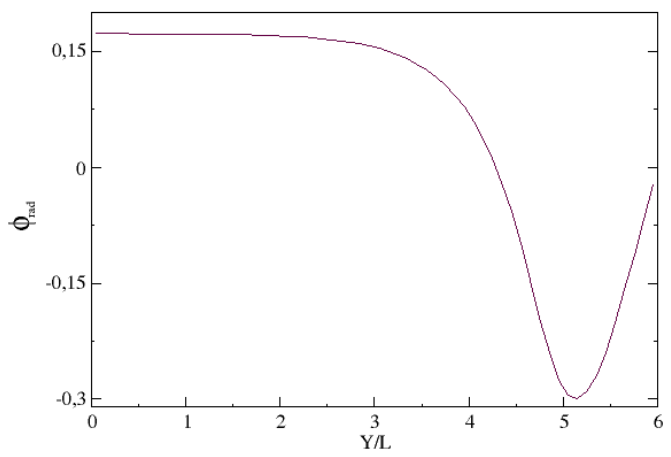

(b)

Figura 4.7: Variação do ângulo do diretor no L-canal com $R e=0.026$ e $E r=5$ e condição de contorno $\phi=0^{\circ}$. (a) $y=5.55$ e $0 \leq x \leq 6$; (b) $x=5.55$ e $0 \leq y \leq 6$. 


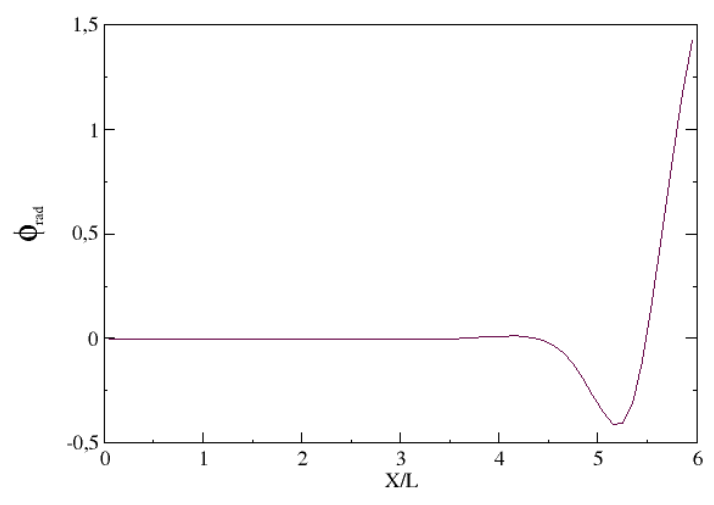

(a)

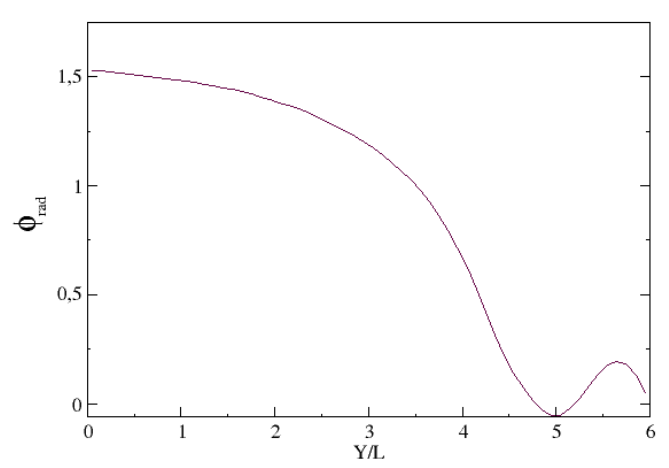

(b)

Figura 4.8: Variação do ângulo do diretor no L-canal com $R e=0.026$ e $E r=5$ e condição de contorno $\phi=90^{\circ}$. (a) $y=5.55$ e $0 \leq x \leq 6$; (b) $x=5.55$ e $0 \leq y \leq 6$.
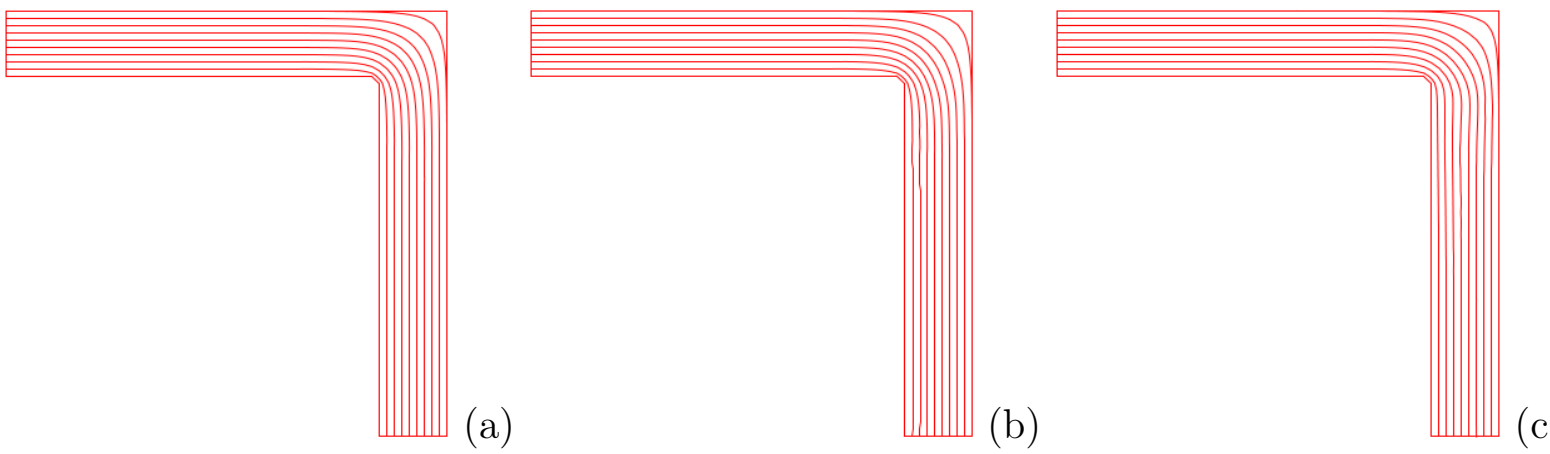

Figura 4.9: Gráficos das linhas de corrente para $\operatorname{Re}=0.026$ e $E r=5$ : (a) $\left(\phi=-90^{\circ}\right)$, (b) $(\phi=0)$ e $(\mathrm{c})\left(\phi=90^{\circ}\right)$. 


\subsubsection{Estudo do número de Ericksen e mudança de geometria do canal}

Para investigar o efeito do número de Ericksen $\operatorname{Er}$ e o efeito da mudança de geometria do L-canal, $R i=L 1 / L$, no escoamento, realizou-se várias simulações utilizando $R e=0.026 \mathrm{e}$ $E r=10,50,100,150,200$ e 250; e variou-se a razão $R i$. Foram considerados os valores $R 2=0.4$ e $R 3=0.3$, o que forneceu um total de 12 simulações. As condições de contorno utilizadas foram aquelas mostradas na Figura 4.1(a). Nessas simulações utilizou os mesmos dados empregados na simulação do efeito das condições de contorno (veja seção 4.1.1).

Um zoom das soluções obtidas, com $R 2=0.4$, para o diretor próximo a entrada do canal de saída é mostrado na Figura 4.10 enquanto que a Figura 4.11 apresenta as linhas de corrente da velocidade no L-canal. Pode-se observar na Figura 4.10 que o diretor satisfaz as condições de contorno impostas para o ângulo $\phi$ para os valores de $E r$ utilizados. As linhas de corrente correspondentes, apresentadas na Figura 4.11, não mostram grandes variações, com exceção das linhas próximas ao canto superior do canal.
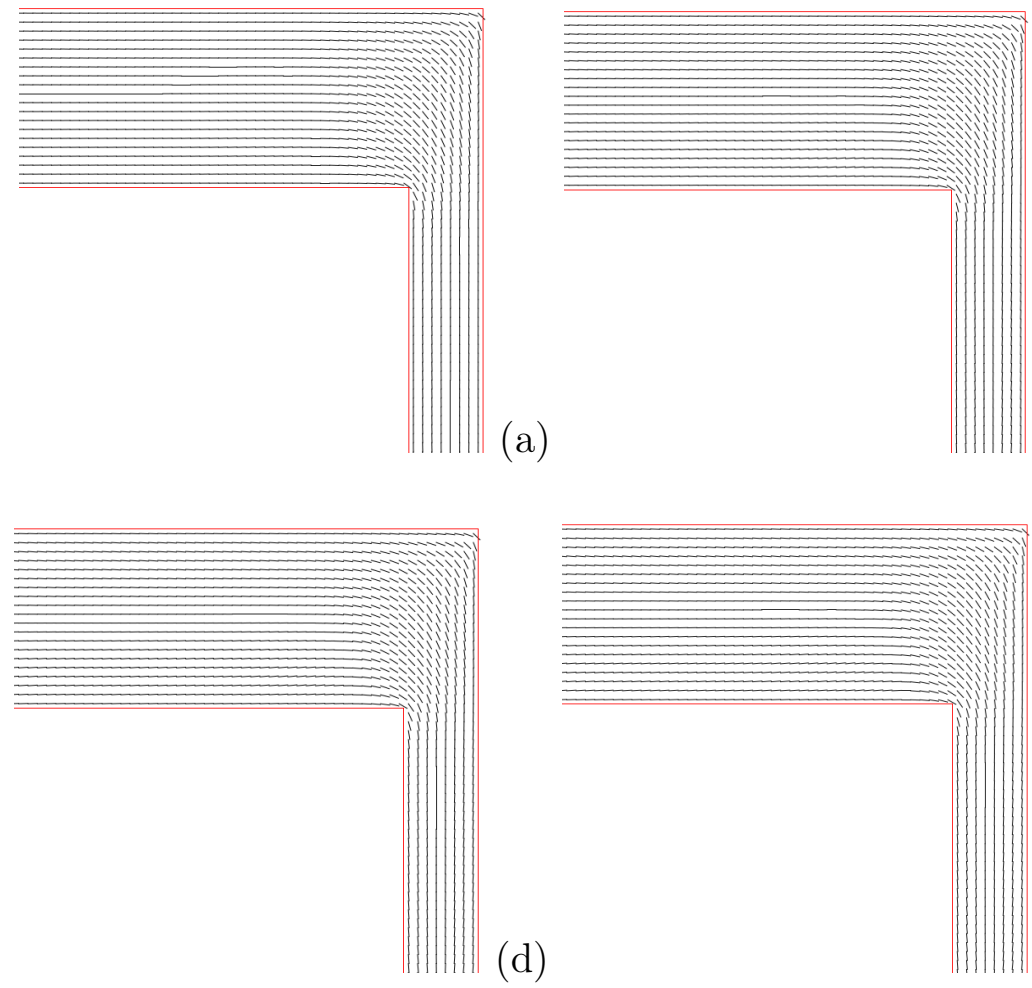

(e)

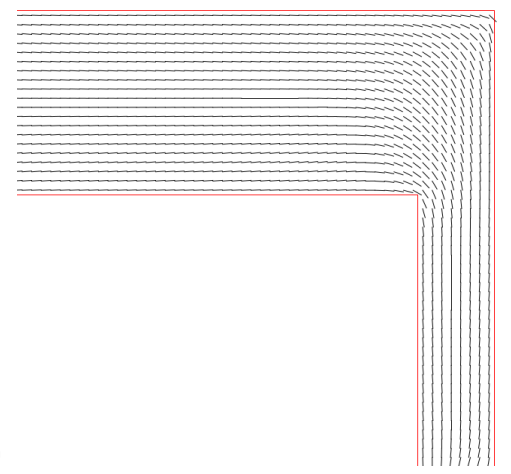

Figura 4.10: Distribuição do diretor no L-canal para $R e=0.026$ e $R 2=0.4$ e diferentes números de Ericksen: (a) $E r=10$, (b) $E r=50$, (c) $E r=100$, (d) $E r=150$, (e) $E r=200$, e (f) $E r=250$.

Com relação aos resultados obtidos com $R 3=0.3$, a Figura 4.12 mostra um zoom do diretor próximo a entrada do canal de saída enquanto as linhas de corrente são apresentadas na Figura 4.13. Pode-se observar que os perfis do diretor, para os números de Ericksen considerados, foram semelhantes aos perfis obtidos para $R 2=0.4$ (ver Figura 4.10). Entretanto, como pode 

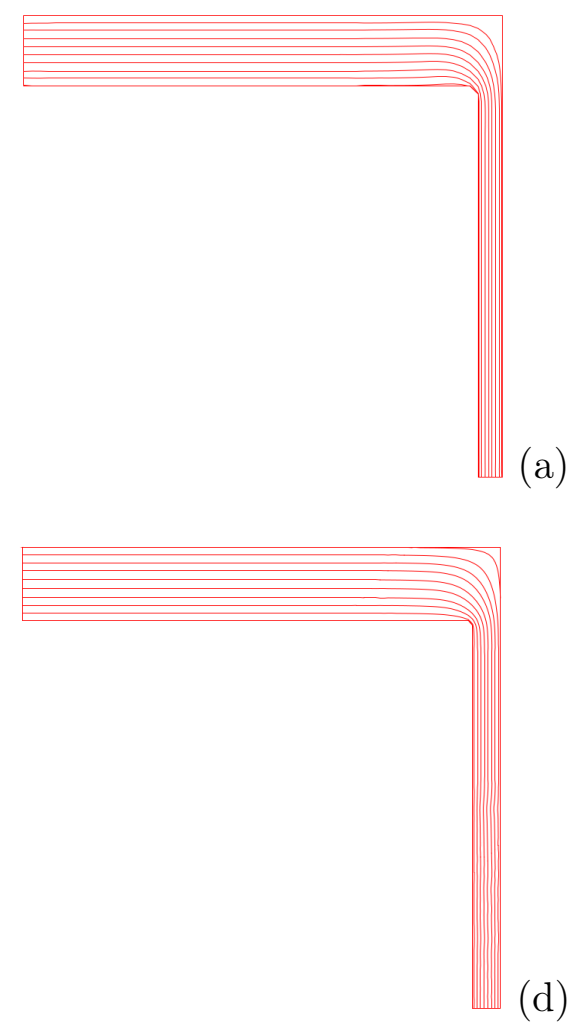
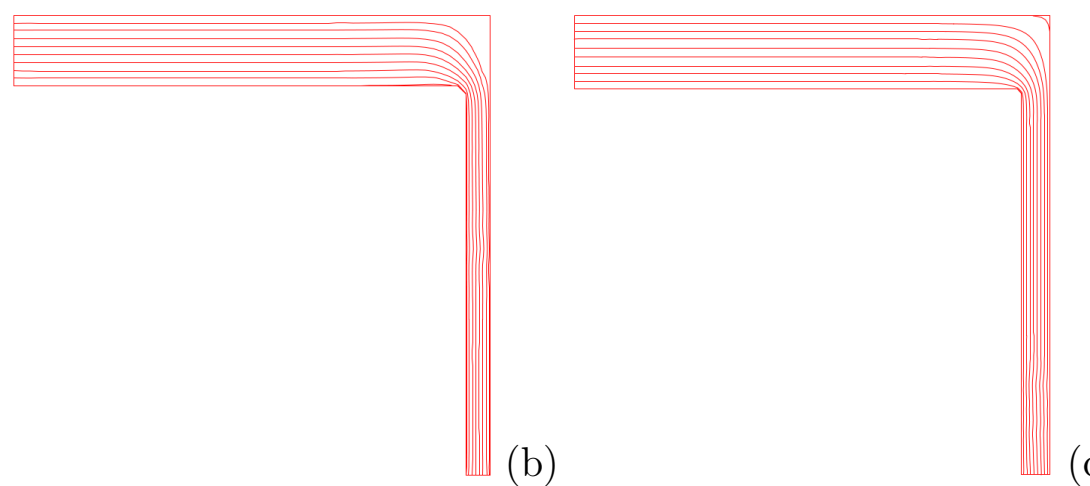

(c)

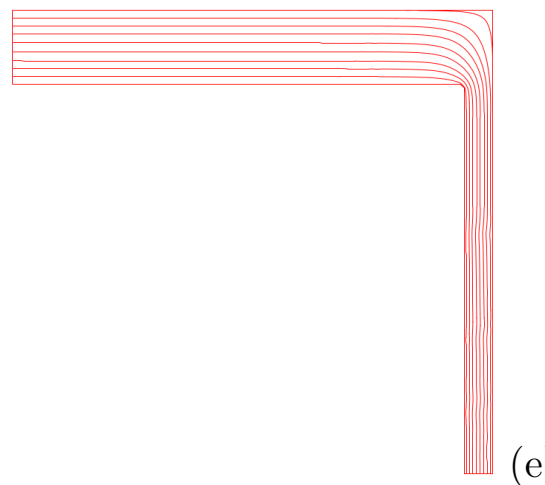

Figura 4.11: Gráficos das linhas de corrente para $R e=0.026$ e $R 2=0.4$ e diferentes números de Ericksen: (a) $E r=10$, (b) $E r=50$, (c) $E r=100$, (d) $E r=150$, (e) $E r=200$, e (f) $E r=250$.

ser visto na Figura 4.13(a) para $E r=10$, o efeito da mudança de geometria ocasionou o surgimento de um "lip vortex" na saída do canal de entrada. Nas figuras subsequentes (veja Figuras 4.13(b)-4.13(f)), observa-se que quando o número de Ericksen é aumentado, o tamanho desse "lip vortex" diminui e desaparece quando $E r=250$. Acreditamos que o aparecimento do "lip vortex" foi devido a mudança de geometria do L-canal e a diminuição da elasticidade (ocasionado pelo aumento de Er) ocasionou a atenuação desse "lip vortex". Um resultado semelhante foi obtido por Chono e Iemoto [24] utilizando o modelo de White Metzner para simular o escoamento de uma solução de polyacrylamid em um L-canal. 

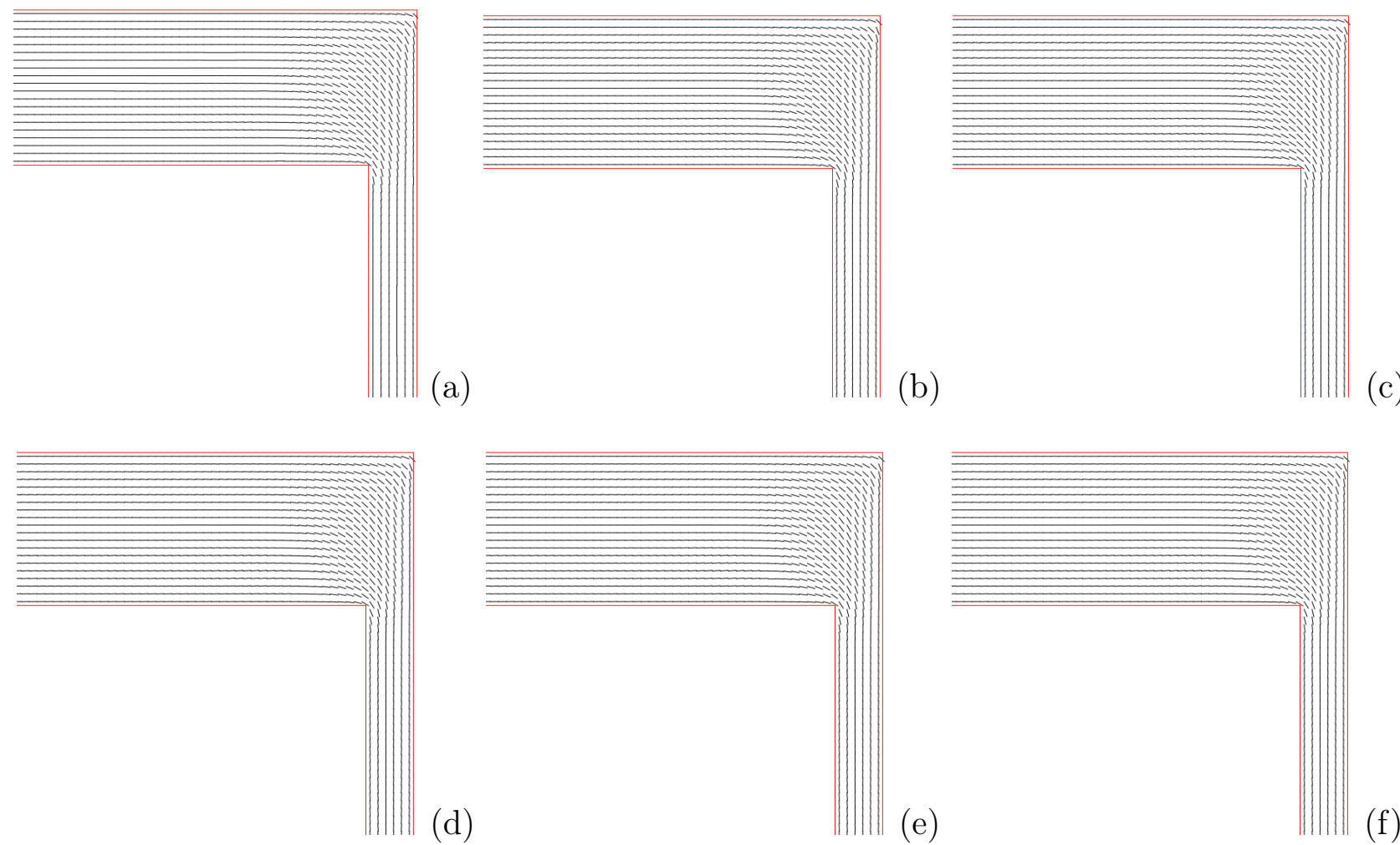

Figura 4.12: Distribuição do diretor no L-canal para $R e=0.026$ e $R 2=0.3$ e vários números de Ericksen: (a) $E r=10$, (b) $E r=50$, (c) $E r=100$, (d) $E r=150$, (e) $E r=200$, e (f) $E r=250$. 

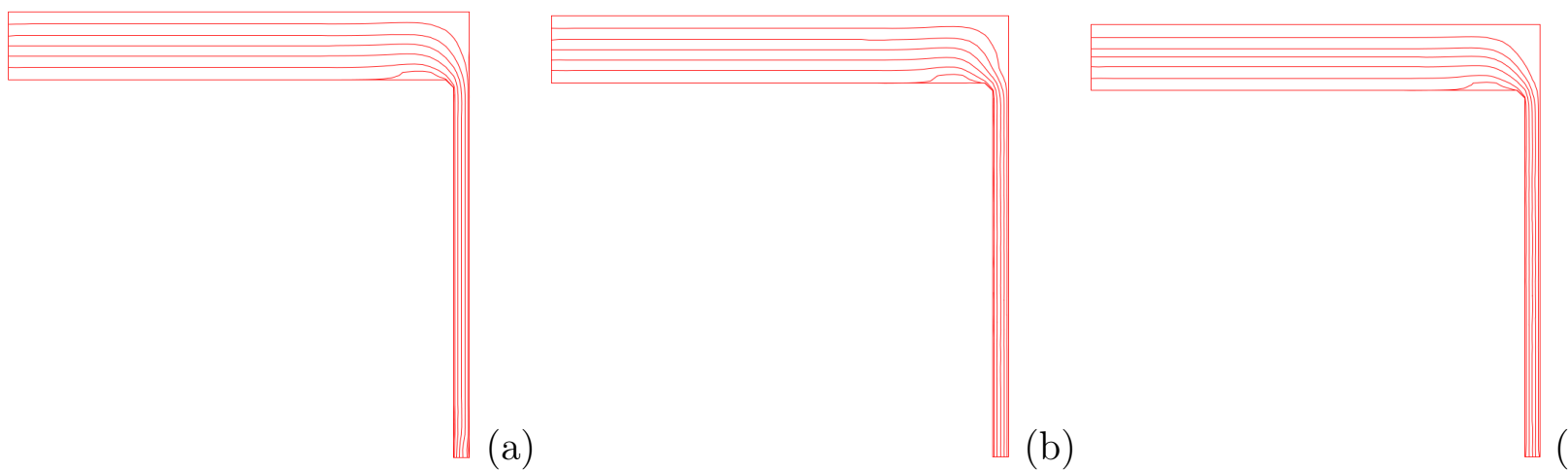

(c)
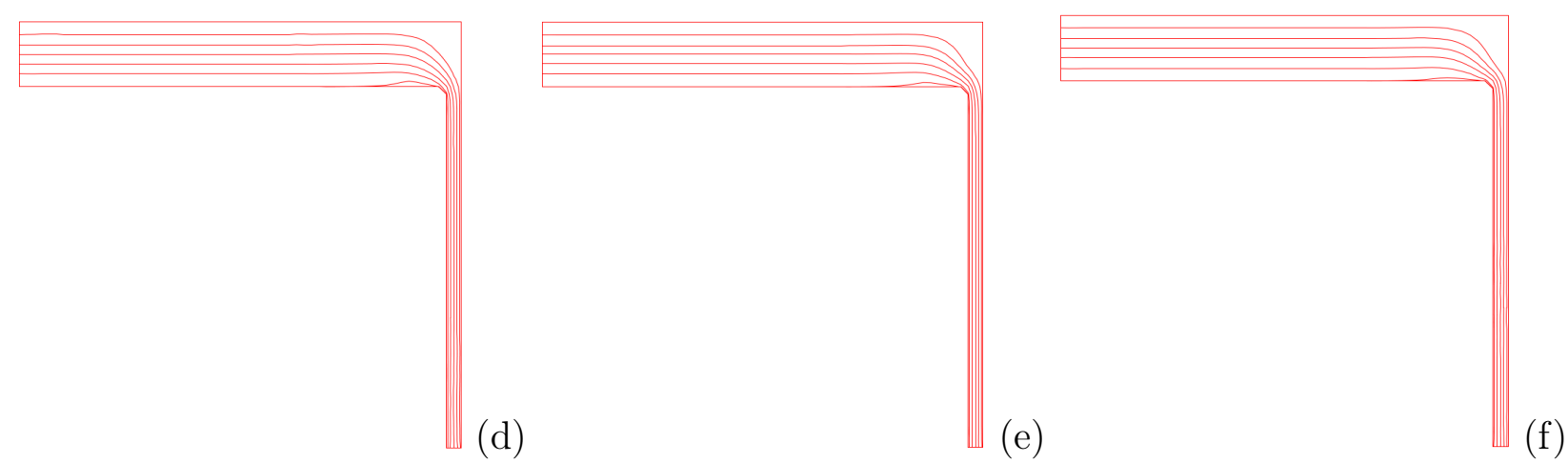

Figura 4.13: Gráficos das linhas de corrente para $R e=0.026$ e $R 3=0.3$ e diferentes números de Ericksen: (a) $E r=10$, (b) $E r=50$, (c) $E r=100$, (d) $E r=150$, (e) $E r=200$, e (f) $E r=250$. 


\subsection{Simulação Numérica do Escoamento através de uma Contração Planar 4:1}

Nesta seção são apresentados resultados numéricos da simulação do escoamento de um cristal líquido nemático através de uma contração planar 4:1. Nesse problema, o fluido escoa por um canal com largura $8 L$ para um outro canal com largura $2 L$, de tal forma que a razão entre as larguras dos canais é 4:1. A geometria do escoamento é mostrada na Figura 4.14.

Esse problema é muito utilizado como " benchmark" no desenvolvimento de métodos numéricos para a simulação de fluidos não-newtonianos $[6,99,126]$. O principal interesse na simulação deste tipo de escoamento vem do fato que fluidos não-newtonianos apresentam comportamento variado quando sujeitos à geometrias complexas, como por exemplo uma contração planar. Um efeito bastante estudado é o tamanho dos vórtices nos cantos e de vórtices que são gerados próximo as paredes verticais na entrada da contração (conhecidos como lip vórtices). O comprimento desses vórtices pode ser afetado pelos números de Reynolds e de Ericksen, o tipo de contração bem como pelas propriedades reológicas de cada material tais como viscosidade e constantes elásticas. Existem vários trabalhos que estudaram numericamente o comportamento de fluidos viscoelásticos em uma contração 4:1 [142, 140], bem como diversos trabalhos experimentais mostrando que o efeito da viscoelasticidade produz padrões de vorticidade diferentes quando sujeitos a esses tipos de geometria (ver Boger et al. [13]).

As condições de contorno para o campo de velocidades foram especificadas na Seção 3.2.1 enquanto que na entrada do canal a velocidade foi especificada por um perfil completamente desenvolvido dado pela equação (3.85).

\section{Definição das condições de contorno para o ângulo $\phi$}

As condições de contorno para o ângulo $\phi$ foram especificadas como segue abaixo, para mais detalhes veja a Figura 4.14:

1. Ao longo das paredes horizontais, o ângulo de ancoragem é nulo e, desse modo obtém-se alinhamento paralelo as paredes.

2. Na entrada de contração, foram utilizados:
(a) $\phi=-45^{\circ}$ em $x=C$ e $y=5 L$;
(b) $\phi=45^{\circ}$ em $x=C$ e $y=3 L$ (detalhes na Figura 4.14).

3. Nas paredes verticais, ou seja, nas paredes da contração, foram empregados:

(a) $\phi=-90^{\circ}$ em $x=C$ e $5 L \leq y \leq 8 L$;

(b) $\phi=90^{\circ}$ em $x=C$ e $0 \leq y \leq 3 L$.

4. Na entrada do canal definido por $x=0$ e $0 \leq y \leq 8 L$, tomou-se $\phi=0$ enquanto que na saída do canal, $x=2 C$ e $3 L \leq y \leq 5 L$, adotou-se $\phi_{, x}=0$. 
(a)

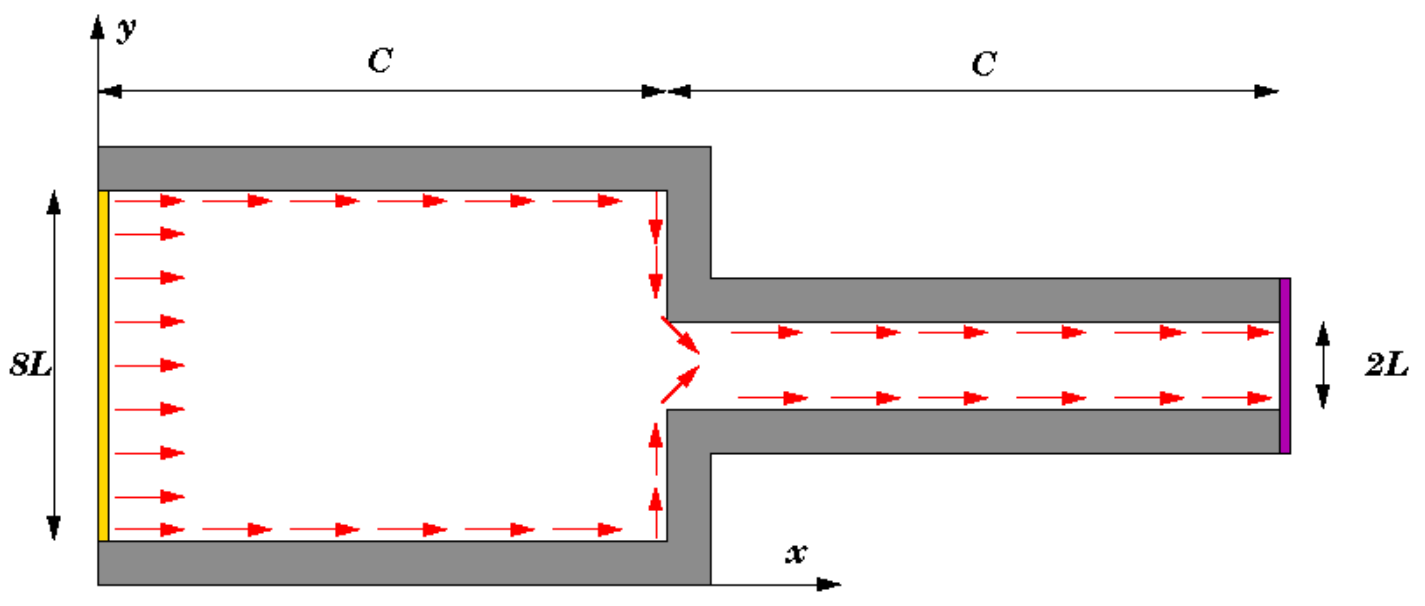

(b)

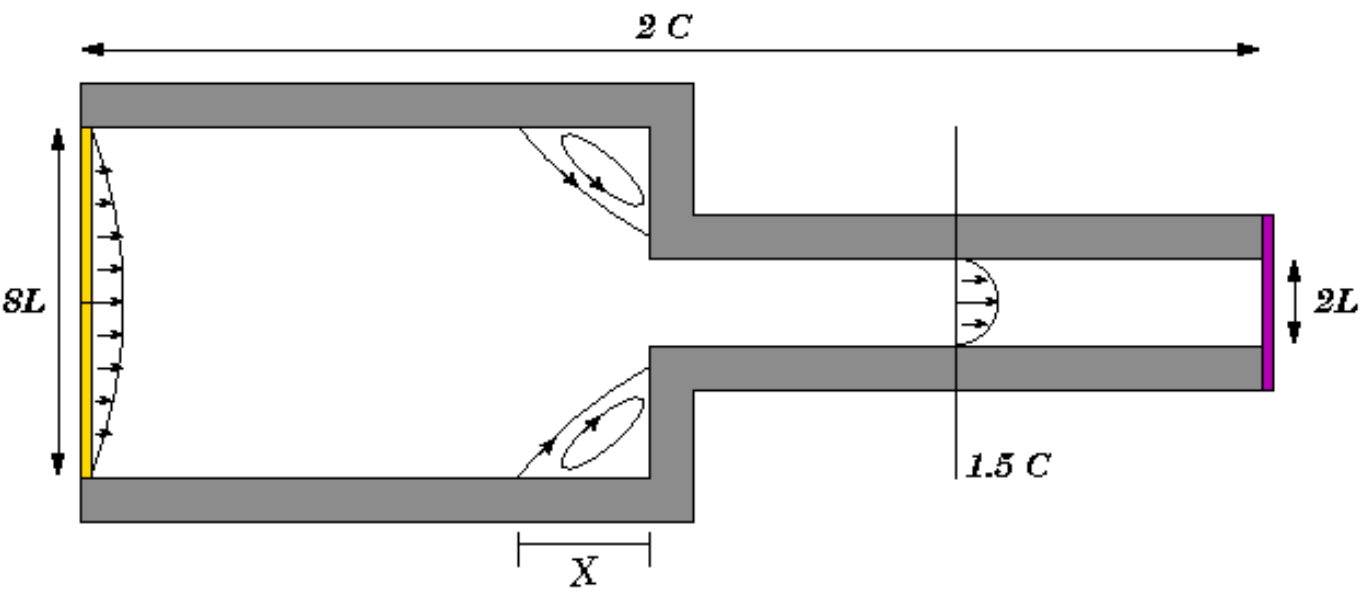

Figura 4.14: Descrição do domínio para a simulação do escoamento em uma contração 4:1. (a) As setas vermelhas representam as condições de contorno utilizadas para o cálculo do ângulo $\phi$ dado pela equação (3.36). (b) $X$ é o comprimento dos vórtices de canto (Lvortex).

Os parâmetros físicos específicos para o cristal líquido nemático MBBA a $25^{\circ}$ empregado para simular este problema são apresentados nas Tabelas A.1, A.2 e A.3 do apêndice A. Os parâmetros restantes que especificam o escoamento são:

- Viscosidade: $\eta=\frac{1}{2} \alpha_{4}=0.0413$ Pa.s;

- Malha: $200 \times 40$ células $(\delta x=\delta y=0.0001) \mathrm{m}$;

- Largura da entrada do canal: $8 L=0.004 m$;

- Comprimento dos canais: $C=0.01 \mathrm{~m}$;

- Largura da saída do canal: $2 L=0.001 \mathrm{~m}$;

- Escala de Comprimento: $L=0.0005 \mathrm{~m}$; 
- Escalas de velocidade: $U_{1}=0.001 \mathrm{~ms}^{-1}, U_{2}=0.002 \mathrm{~ms}^{-1}$ e $U_{3}=0.005 \mathrm{~ms}^{-1}$. Essas velocidades foram utilizadas para especificar a velocidade na entrada do canal, conforme a equação (3.85).

Os números de Reynolds foram calculados utilizando metade da largura da saída do canal $L$, a viscosidade $\eta$ e as escalas de velocidade $U_{1}, U_{2}$ e $U_{3}$ e assim foram obtidos $R e=0.013$, $R e=0.026$ e $R e=0.066$, respectivamente. Nos resultados apresentados a seguir, os seguintes números de Ericksen foram empregados:

\begin{tabular}{c|l|l|l}
\hline Número de Ericksen & \multicolumn{3}{|c}{ Constantes Elásticas } \\
\hline$E r$ & $R e=0.013$ & $R e=0.026$ & $R e=0.066$ \\
\hline 5 & $K=4.130 \times 10^{-9}$ & $K=8.260 \times 10^{-9}$ & $K=2.065 \times 10^{-8}$ \\
10 & $K=2.065 \times 10^{-9}$ & $K=4.130 \times 10^{-9}$ & $K=1.033 \times 10^{-8}$ \\
20 & $K=1.033 \times 10^{-9}$ & $K=2.065 \times 10^{-9}$ & $K=5.163 \times 10^{-9}$ \\
30 & $K=6.883 \times 10^{-10}$ & $K=1.377 \times 10^{-9}$ & $K=3.442 \times 10^{-9}$ \\
40 & $K=5.163 \times 10^{-10}$ & $K=1.033 \times 10^{-9}$ & $K=2.581 \times 10^{-9}$ \\
50 & $K=4.130 \times 10^{-10}$ & $K=8.260 \times 10^{-10}$ & $K=2.065 \times 10^{-9}$ \\
70 & $K=2.950 \times 10^{-10}$ & $K=5.900 \times 10^{-10}$ & $K=1.475 \times 10^{-9}$ \\
100 & $K=2.065 \times 10^{-10}$ & $K=4.130 \times 10^{-10}$ & $K=1.033 \times 10^{-9}$ \\
150 & $K=1.377 \times 10^{-10}$ & $K=2.753 \times 10^{-10}$ & $K=6.883 \times 10^{-10}$ \\
200 & $K=1.033 \times 10^{-10}$ & $K=2.065 \times 10^{-10}$ & $K=5.163 \times 10^{-10}$ \\
\hline
\end{tabular}

A seguir são apresentados os resultados obtidos nessas simulações.

Com o objetivo de observar os efeitos elásticos e viscosos (tais como a diminuição do tamanho dos vórtices) no escoamento, o problema da contração foi simulado para os valores dos números de Reynolds e de Ericksen dados na Tabela acima até que o estado estacionário foi atingido.

De fato, para ilustrar que a solução estacionária foi encontrada, as velocidades $u$ e $v$ e a pressão $p$ para $R e=0.013$ e $E r=10$ são apresentadas na Figura 4.15. Pode-se observar na Figura 4.15 que a componente $v$ da velocidade é nula em quase todo o domínio, a componente $u$ da velocidade apresenta perfil parabólico no canal de saída e que a pressão, varia apenas como uma função de $x$. Esses resultados indicam que o estado estacionário foi atingido. Para confirmar esse fato, a Figura 4.16(a) mostra a variação da pressão na linha central do canal, $y c=4.1$ e a Figura 4.16(b) ilustra os resultados obtidos para $p_{, x}\left(y_{c}, x\right)$. O perfis das velocidades $u\left(x_{1}, y\right), v\left(x_{1}, y\right), u\left(x_{2}, y\right), v\left(x_{2}, y\right)$ e $u\left(x_{3}, y\right), v\left(x_{3}, y\right)$, em que $x_{1}=10.1, x_{2}=19.9, x_{3}=$ 30.1 são mostrados na Figura 4.17. Pode-se observar na Figura 4.16 que, com exceção de pontos próximos a entrada da contração, a pressão varia linearmente com $x$ e a Figura 4.17 mostra que, com exceção de pontos próximos a entrada da contração, a velocidade $v$ é nula e 
a velocidade $u$ tem perfil parabólico. Mais ainda, a Figura 4.18(a) mostra o perfil do ângulo $\phi\left(y_{c}, x\right)$ enquanto que a Figura 4.18(b) fornece uma comparação dos valores obtidos em $\phi\left(x_{3}, y\right)$ com a solução analítica para escoamentos totalmente desenvolvidos (ver equação (3.78)). A Figura 4.18(a) mostra que o ângulo $\phi\left(y_{c}, x\right)$ assume valores pequenos na linha no centro do canal e a Figura 4.18(b) mostra que a solução numérica obtida concorda com a solução analítica para escoamentos totalmente desenvolvidos. Portanto, os resultados mostrados nas Figuras 4.16, 4.17 e 4.18 confirmam que, nessa simulação, o estado estacionário foi alcançado.

Para os demais valores dos números de Reynolds e Ericksen, os perfis da velocidade e pressão obtidos foram semelhantes.

Um zoom das soluções obtidas para o diretor e as respectivas linhas de corrente próximas a entrada da contração para $R e=0.013$ são apresentadas nas Figuras 4.19 e 4.20 enquanto as Figuras 4.21 e 4.22 mostram os resultados obtidos utilizando $R e=0.026$, e os resultados obtidos com $R e=0.066$ são apresentados nas Figuras 4.23 e 4.24. Pode-se observar nas Figuras 4.19(a), 4.19(b), 4.19(c) para $E r=10,50$ e 70 ( $R e=0.013)$, que o diretor concorda com as condições de contorno impostas. Os resultados obtidos para o diretor com $E r=100,150$, e 200 (veja as Figuras 4.19(d), 4.19(e), 4.19(f)) foram semelhantes aos resultados obtidos com os números de Ericksen menores, ou seja, concordam com as condições de contorno impostas. A principal diferença ocorre com os diretores na entrada da contração e após a contração onde, pode-se observar que o diretor começa a convergir para a linha central do canal.

Os resultados obtidos para o perfil do diretor para $R e=0.026$ e $R e=0.066$ foram semelhantes aos resultados obtidos com $R e=0.013$ como pode ser visto nas Figuras 4.21 e 4.23, respectivamente.

As correspondentes linhas de corrente apresentadas nas Figuras 4.20, 4.22 e 4.24 são interessantes e mostram que o vórtice de canto diminui à medida que o número de Ericksen aumenta. Estes resultados mostram que se o números de Reynolds está fixo então o tamanho do vórtice de canto diminui se o número de Ericksen é aumentado, isso equivale dizer, que o tamanho do vórtice de canto diminui se a constante elástica é diminuída. A Tabela 4.1 mostra o tamanho dos vórtices obtidos nas simulações e a Figura 4.25 ilustra a variação do comprimento do vórtice de canto à medida que o número de Ericksen é aumentado. Além disso, acreditamos que a diminuição do tamanho do vórtice de canto foi devido aos efeitos viscoelásticos. De fato, esse efeito tem sido mencionado em trabalhos na comunidade científica que trabalha com simulação numérica e experimental de escoamentos viscoelásticos (veja por exemplo [93, 104, 114, 130]). 

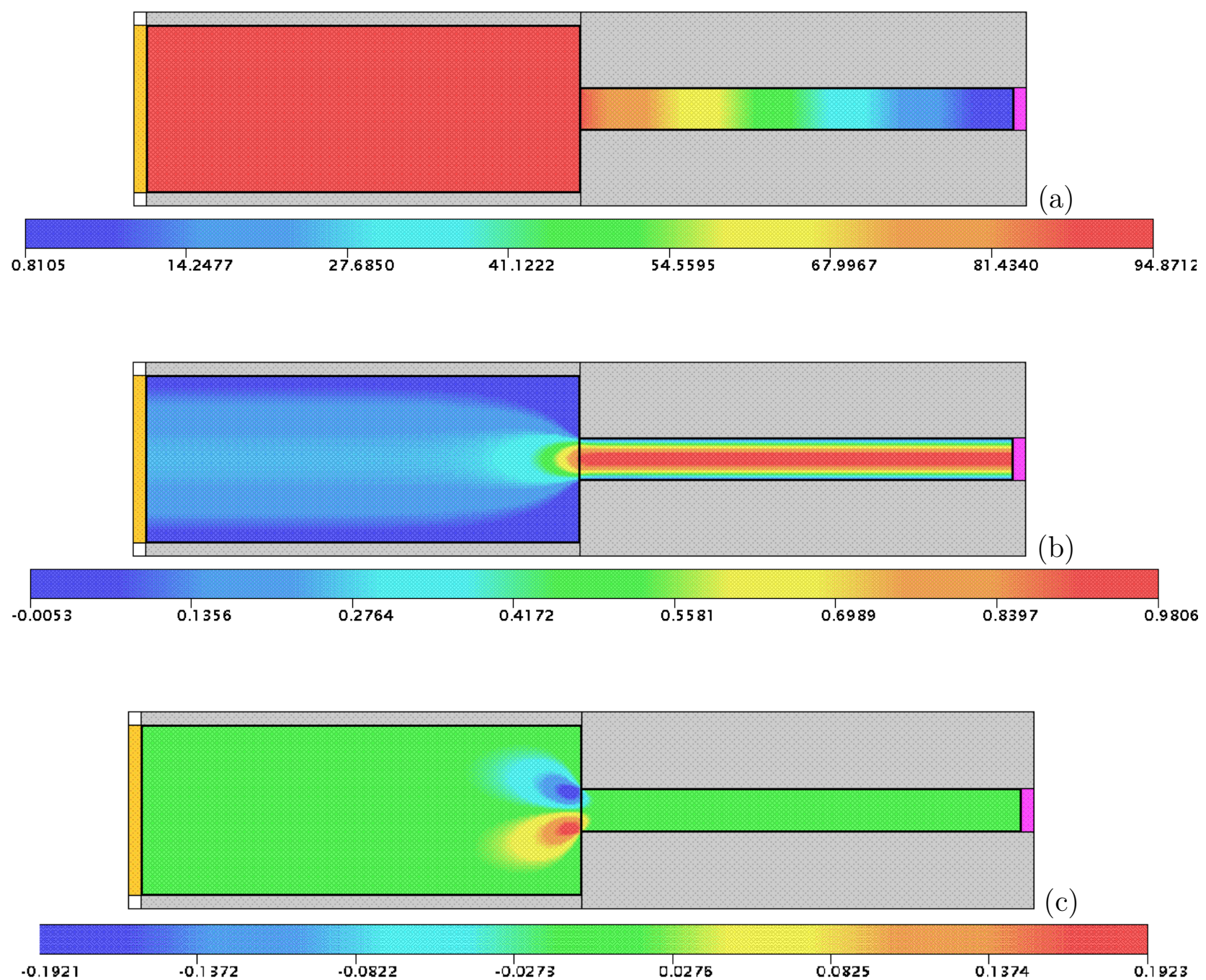

Figura 4.15: Simulação numérica do escoamento através de uma contração com $R e=0.013$ e $E r=10$. Isolinhas: (a) pressão, (b) componente $u$ e (c) componente $v$ da velocidade.

\subsection{Simulação Numérica do Escoamento de um Cristal Líquido Nemático em uma Expansão}

Nesta seção são apresentados resultados numéricos obtidos na simulação do escoamento de um cristal líquido nemático em uma expansão planar 1:4 (veja Figura 4.26). Para esse problema, o fluido escoa em um canal com largura $2 L$, para um outro canal com largura $8 L$. Será realizado um estudo do comportamento de vórtices gerados nos cantos e de vórtices gerados nas paredes da expansão ("lip vortices") em consequência do aumento dos números de Reynolds e de Ericksen. Com o objetivo de estudar o efeito da viscosidade nemática no escoamento, serão empregados dois tipos de cristais líquidos nemáticos MBBA nessas simulações . 
(a)

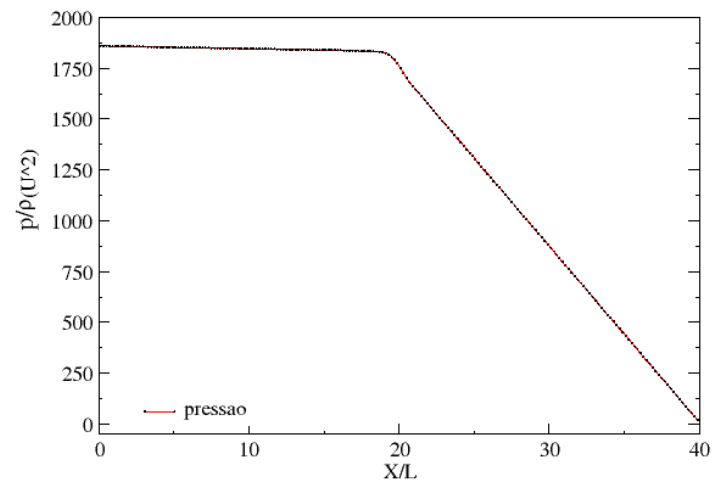

(b)

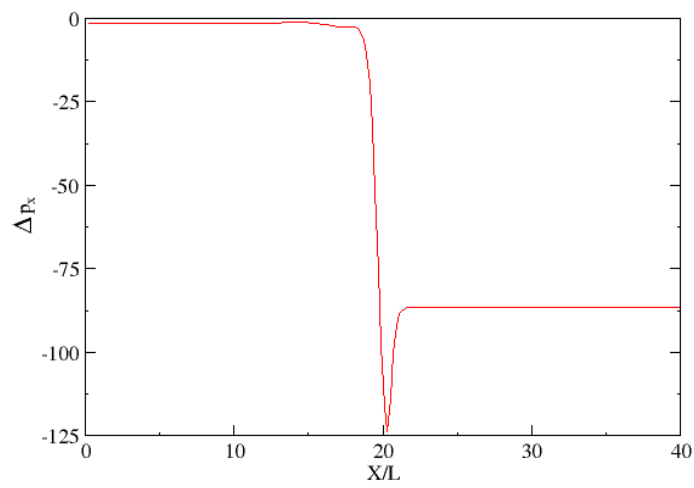

Figura 4.16: Variação da pressão e do gradiente de pressão através de uma contração com $R e=0.013$ e $E r=10$. (a) pressão, (b) gradiente de pressão.

(a)

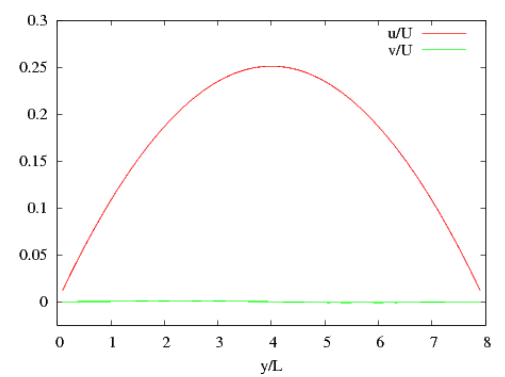

(b)

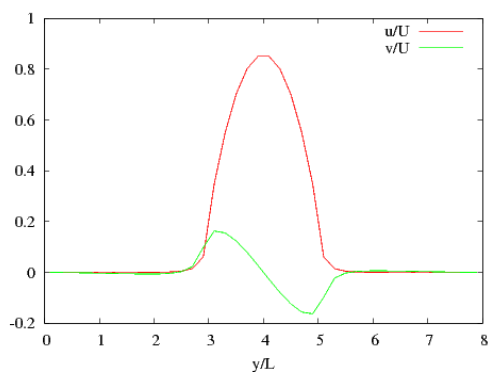

(c)

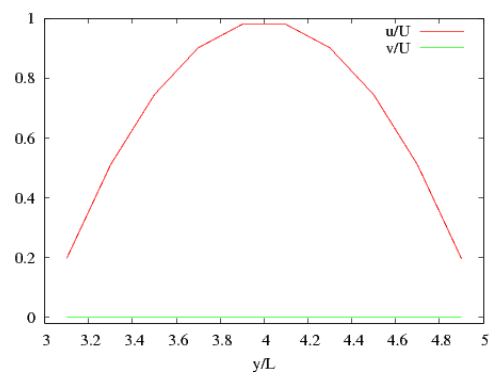

Figura 4.17: Perfis das velocidades $u$ e $v$ em diferentes seções do canal com $R e=0.013$ e $E r=10$. (a) $x=10.1$, (b) $x=19.9$ e (c) $x=30.1$.

(a)

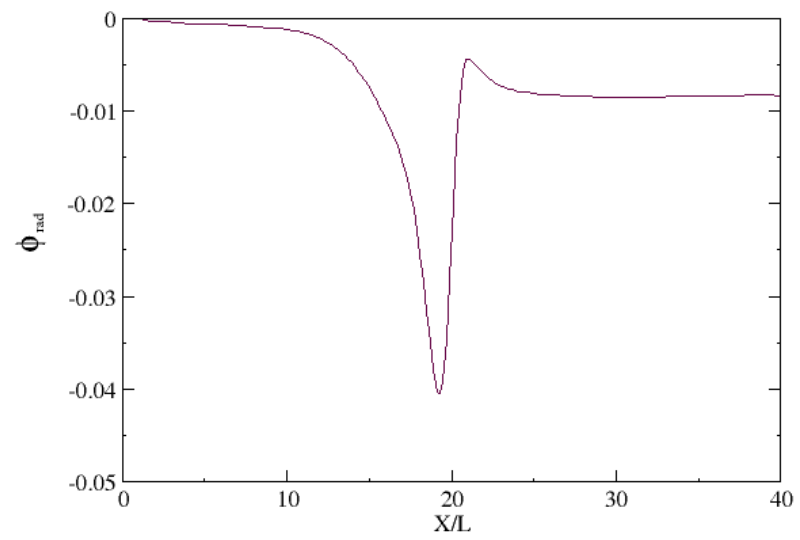

(b)

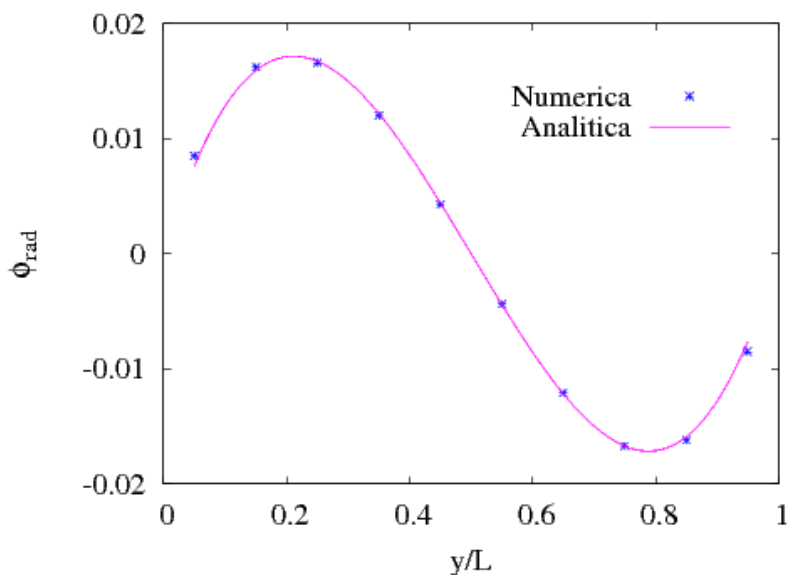

Figura 4.18: Simulação através de uma contração com $R e=0.013$ e $E r=10$. (a) Variação do ângulo do diretor $\phi$ na direção $x$ para $y_{c}=4.1$. (b) Comparação das soluções numérica e analítica do ângulo do diretor $\phi$ para $x=30.1$. 
(a)

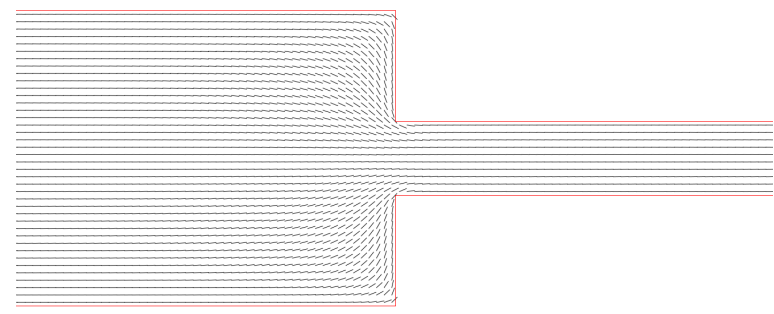

(c)

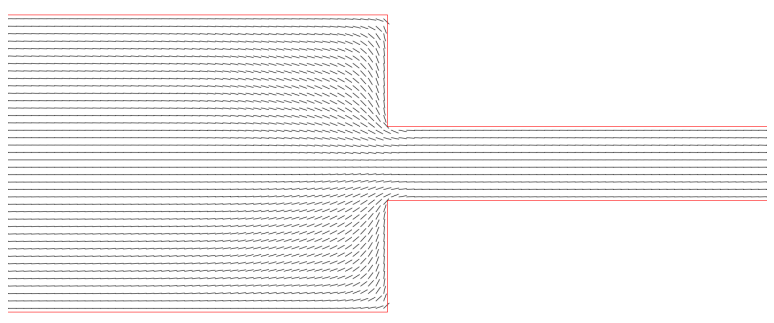

(e)

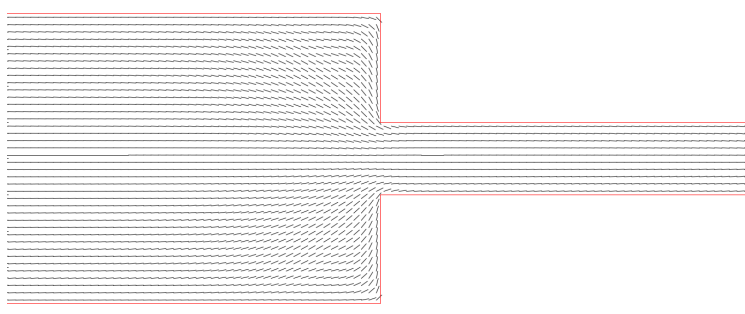

(d)

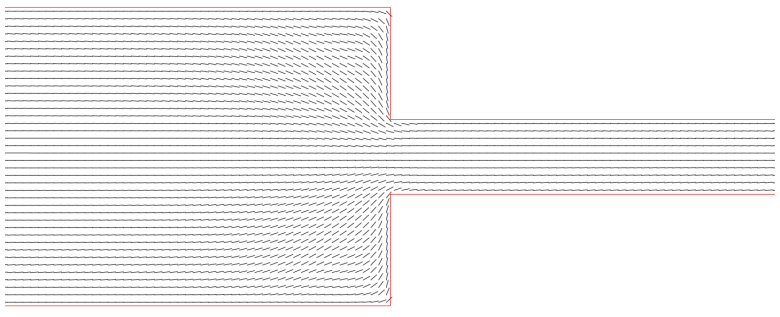

(b)

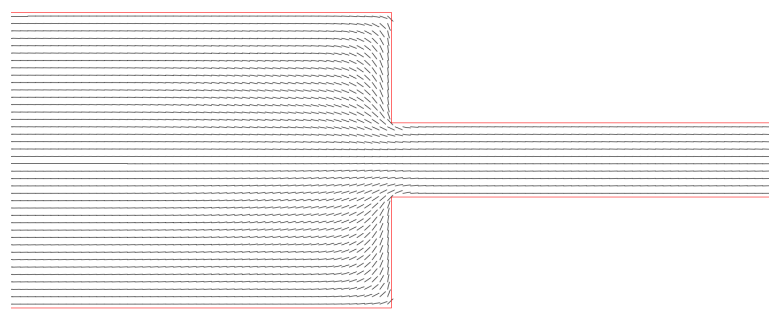

(f)

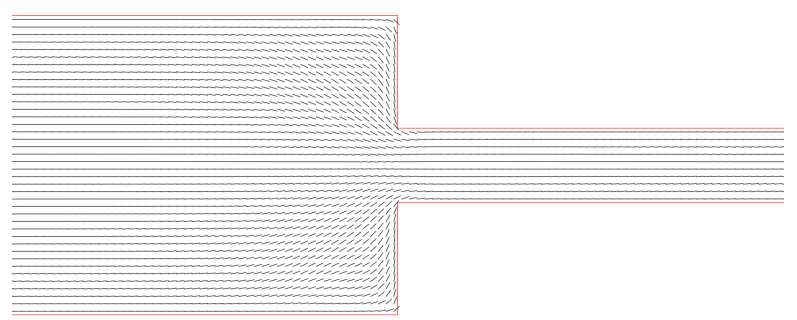

Figura 4.19: Simulação numérica do diretor através de uma contração planar 4:1 com $R e=$ 0.013. Gráficos do diretor para diferentes números de Ericksen: (a) $E r=10$, (b) $E r=50$, (c) $E r=70,(d) E r=100$, (e) $E r=150$ e (f) $E r=200$. 


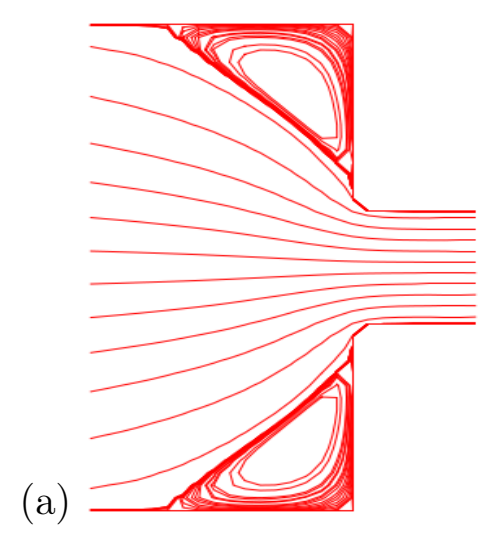

(b)

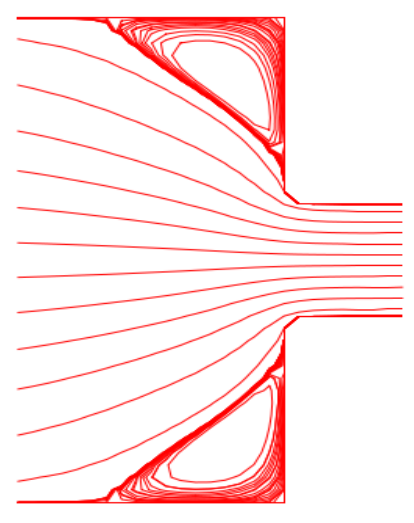

(c)

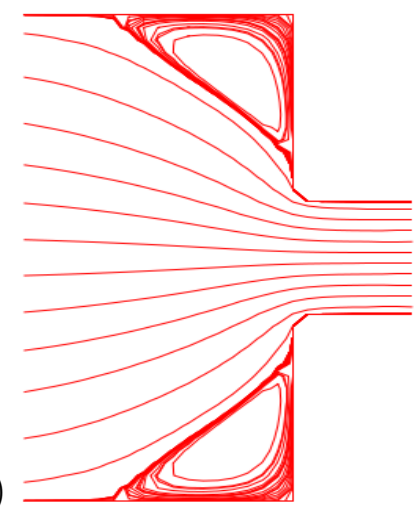

(d)

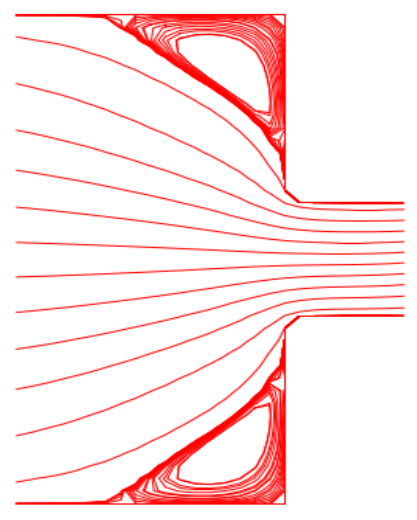

(e)

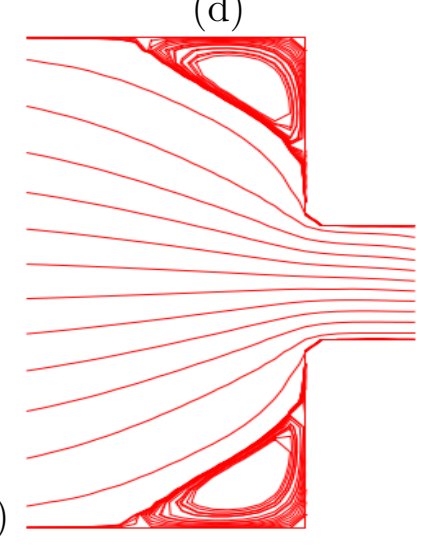

(f)

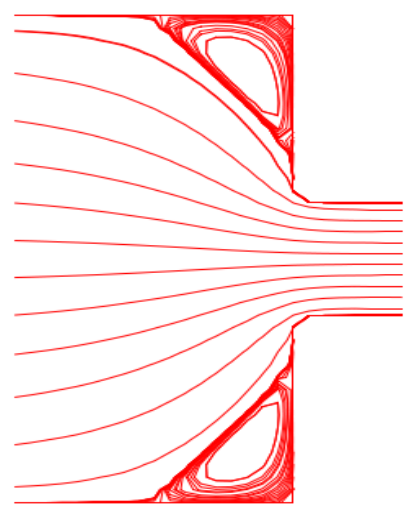

Figura 4.20: Gráficos das linhas de corrente para $R e=0.013$ para diferentes números de Ericksen: (a) $E r=10$, (b) $E r=50$, (c) $E r=70$, (d) $E r=100$, (e) $E r=150$ e (f) $E r=200$. 
(a)

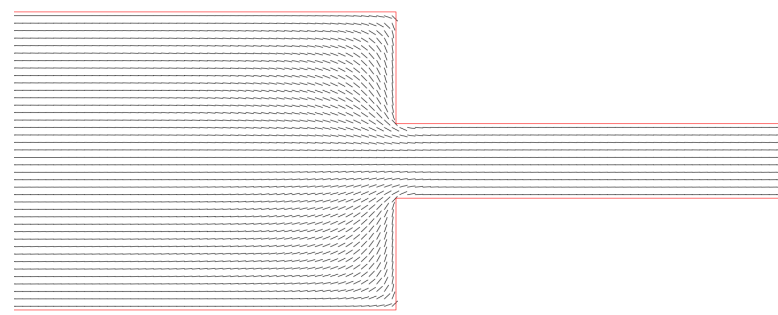

(c)

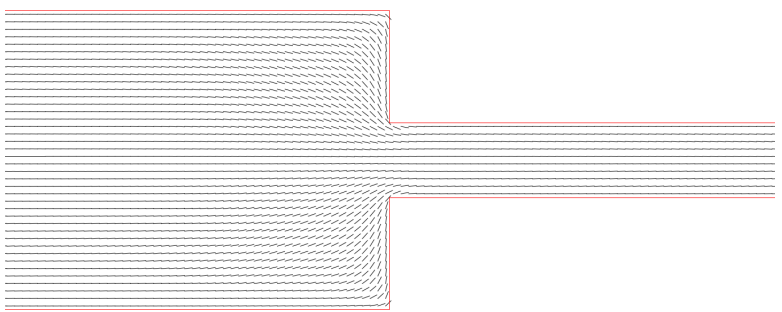

(b)

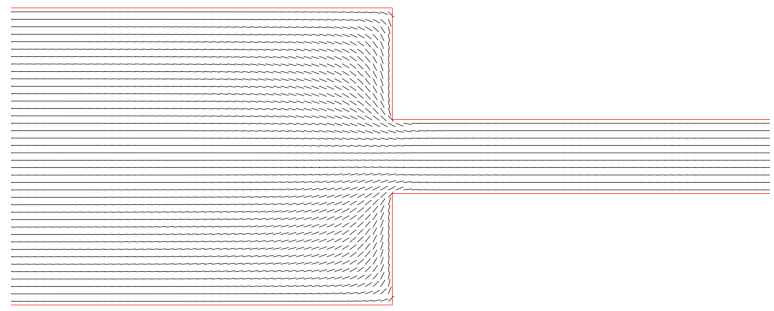

(d)

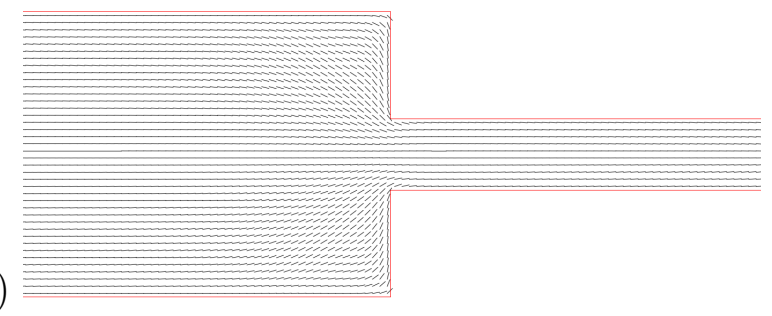

(f)

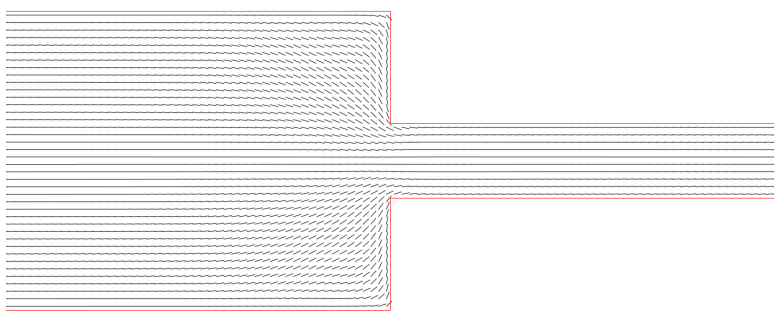

(e)

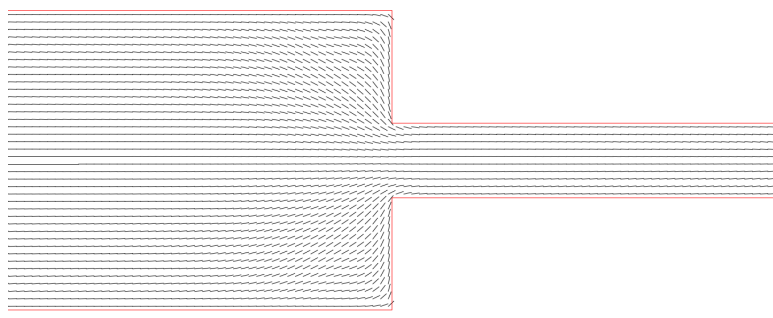

Figura 4.21: Simulação numérica do diretor através de uma contração planar 4:1 com $R e=$ 0.026. Gráficos do diretor para diferentes números de Ericksen: (a) $E r=10$, (b) $E r=50$, (c) $E r=70$, (d) $E r=100$, (e) $E r=150$ e (f) $E r=200$. 
(a)

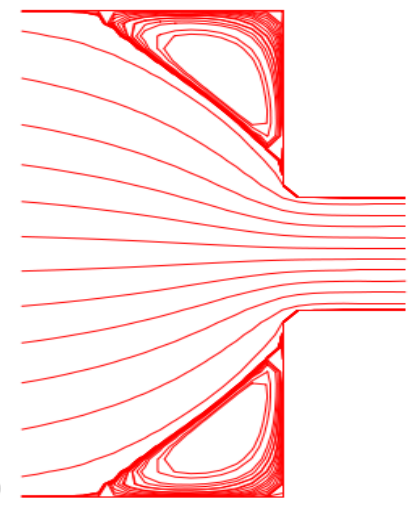

(d)

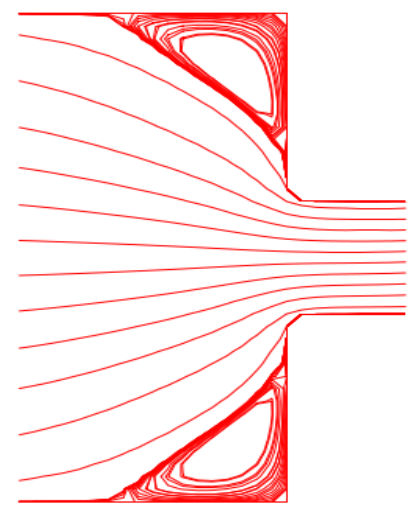

(b)

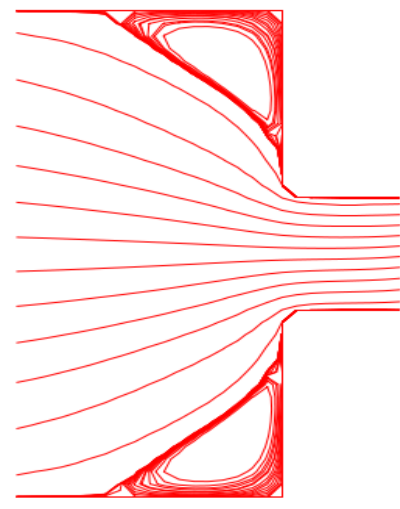

(c)

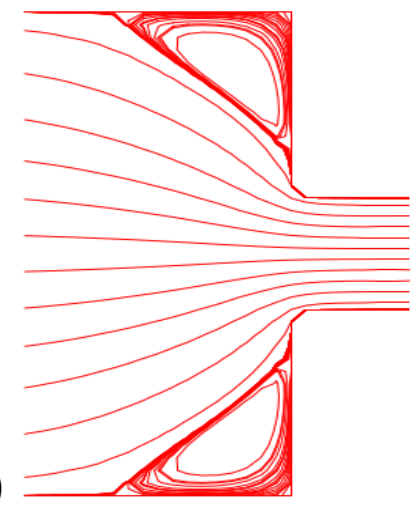

(f)

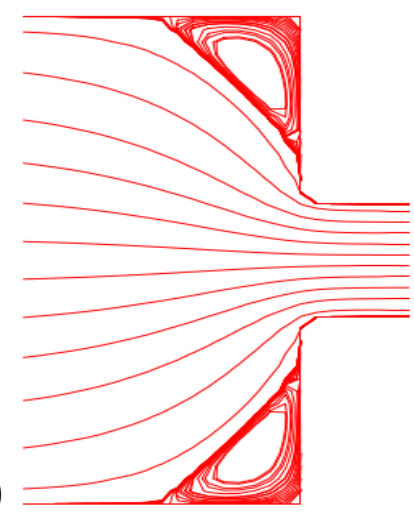

Figura 4.22: Gráficos das linhas de corrente para $R e=0.026$ para diferentes números de Ericksen: (a) $E r=10$, (b) $E r=50$, (c) $E r=70$, (d) $E r=100$, (e) $E r=150$ e (f) $E r=200$. 
(a)

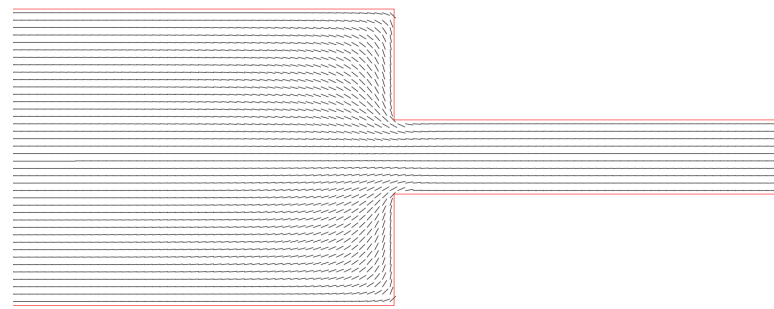

(c)

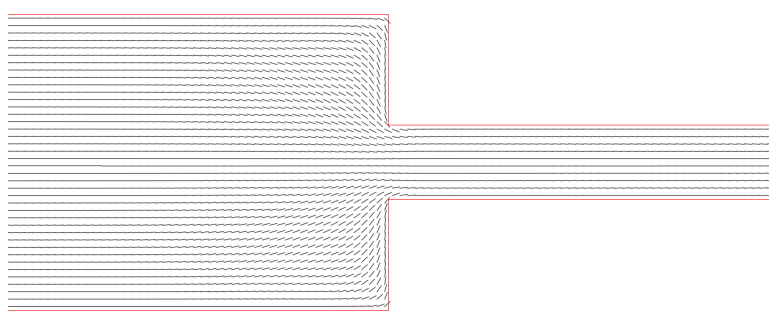

(d)

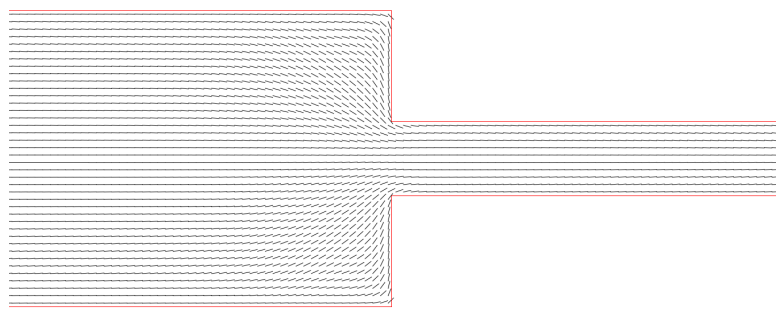

(b)

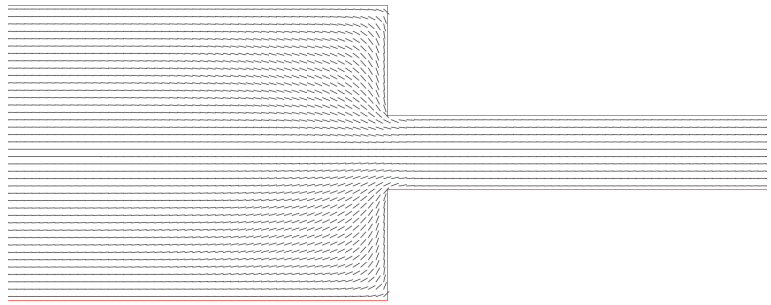

(e)

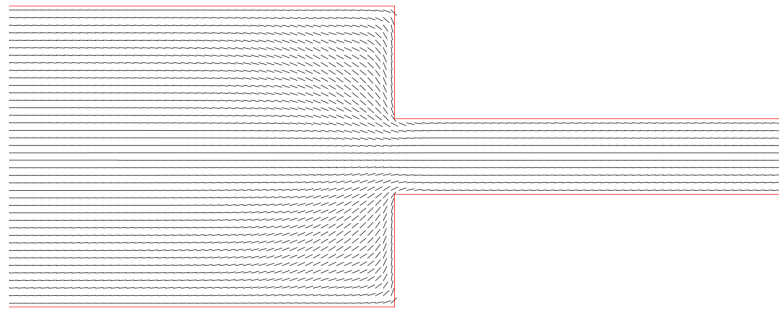

(f)

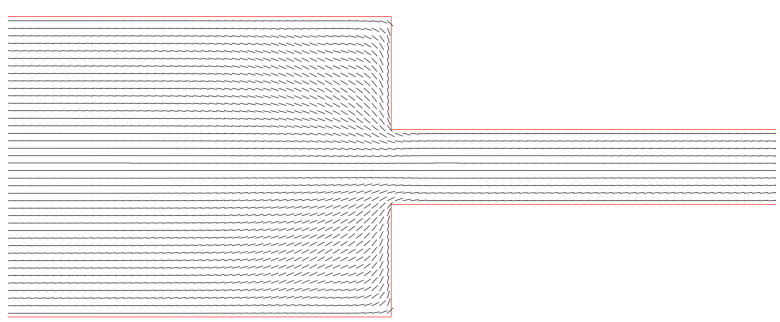

Figura 4.23: Simulação numérica do diretor através de uma contração planar 4:1 com $R e=$ 0.066. Gráficos do diretor para diferentes números de Ericksen: (a) $E r=10$, (b) $E r=50$, (c) $E r=70$, (d) $E r=100$, (e) $E r=150$ e (f) $E r=200$. 
(a)

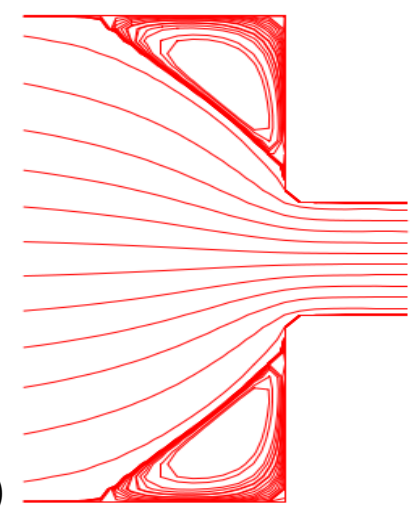

(b)

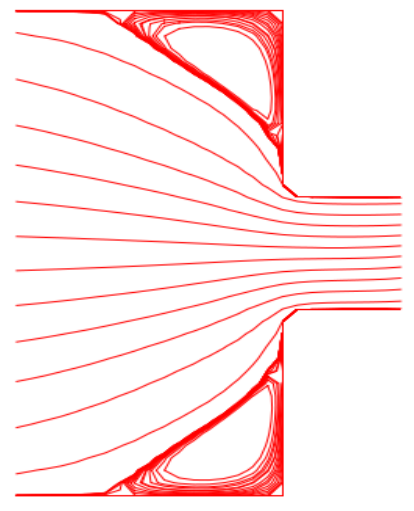

(c)

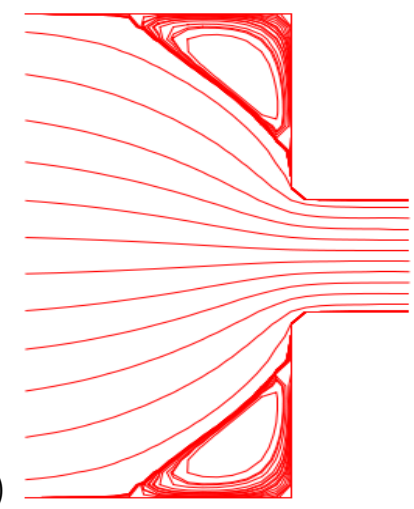

(f)

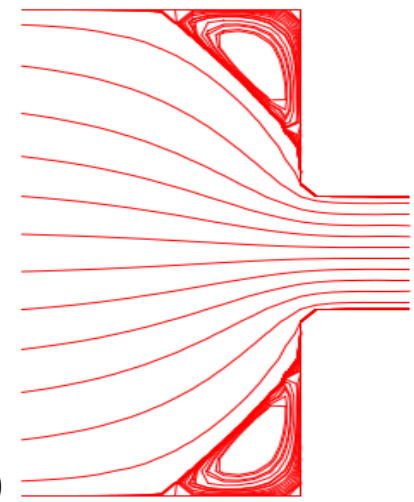

Figura 4.24: Gráficos das linhas de corrente para $R e=0.066$ para diferentes números de Ericksen: (a) $E r=10$, (b) $E r=50$, (c) $E r=70$, (d) $E r=100$, (e) $E r=150$ e (f) $E r=200$.

\begin{tabular}{c|l|l|l}
\hline Número de Ericksen & \multicolumn{3}{|c}{ Comprimento do vórtice - Lvortex } \\
\hline$E r$ & $R e=0.013$ & $R e=0.026$ & $R e=0.066$ \\
\hline 5 & 2.523 & 2.518 & 2.503 \\
10 & 2.516 & 2.509 & 2.496 \\
20 & 2.494 & 2.487 & 2.479 \\
30 & 2.482 & 2.478 & 2.466 \\
40 & 2.468 & 2.459 & 2.447 \\
50 & 2.396 & 2.382 & 2.353 \\
70 & 2.343 & 2.312 & 2.270 \\
100 & 2.276 & 2.243 & 2.187 \\
150 & 2.109 & 2.081 & 2.058 \\
200 & 1.841 & 1.811 & 1.763 \\
\hline
\end{tabular}

Tabela 4.1: Comprimento dos vórtices em função do número de Ericksen. 


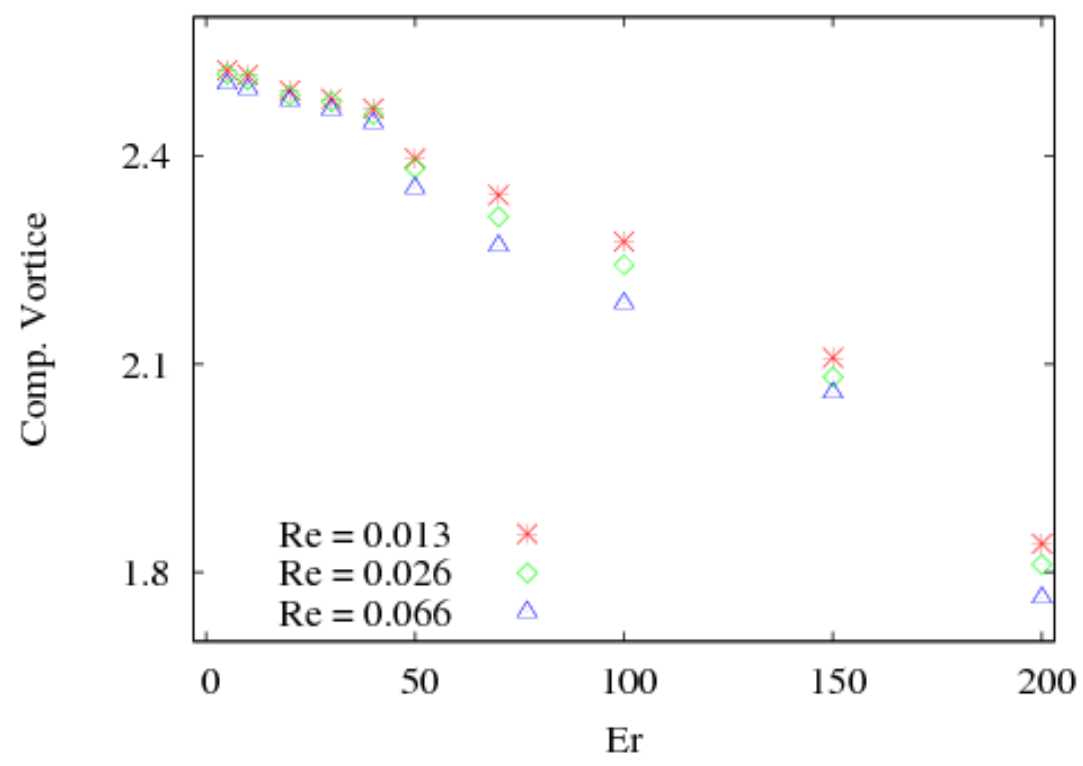

Figura 4.25: Variação do comprimento do vórtice (Lvortex $=X / L$ ver Figura 4.14) em função do número de Ericksen.

As condições de contorno para o campo de velocidade foram especificados na Seção 3.2.1 e a velocidade na entrada do canal foi especificada por um perfil desenvolvido dado pela equação (3.85).

\section{Definição das condições de contorno para o ângulo do diretor $\phi$}

As condições de contorno para o ângulo $\phi$ foram especificadas como segue (para detalhes veja Figura 4.26):

1. Nas paredes horizontais, o ângulo de ancoragem é nulo, e assim tem-se alinhamento paralelo nas paredes.

2. Na entrada da expansão, foram utilizados:

(a) $\phi=45^{\circ}$ em $x=C$ e $y=5 L$;

(b) $\phi=-45^{\circ}$ em $x=C$ e $y=3 L$ (detalhes na Figure 4.26).

3. Nas paredes verticais, foram empregados:

(a) $\phi=-90^{\circ}$ em $x=C$ e $0 \leq y \leq 3 L$;

(b) $\phi=90^{\circ}$ em $x=C$ e $5 L \leq y \leq 8 L$.

4. Na entrada do canal definido por $x=0$ e $3 L \leq y \leq 5 L$, foi imposto $\phi=0$ e na saída do canal, $x=2 C$ e $0 \leq y \leq 8 L$, adotou-se $\phi_{, x}=0$. 


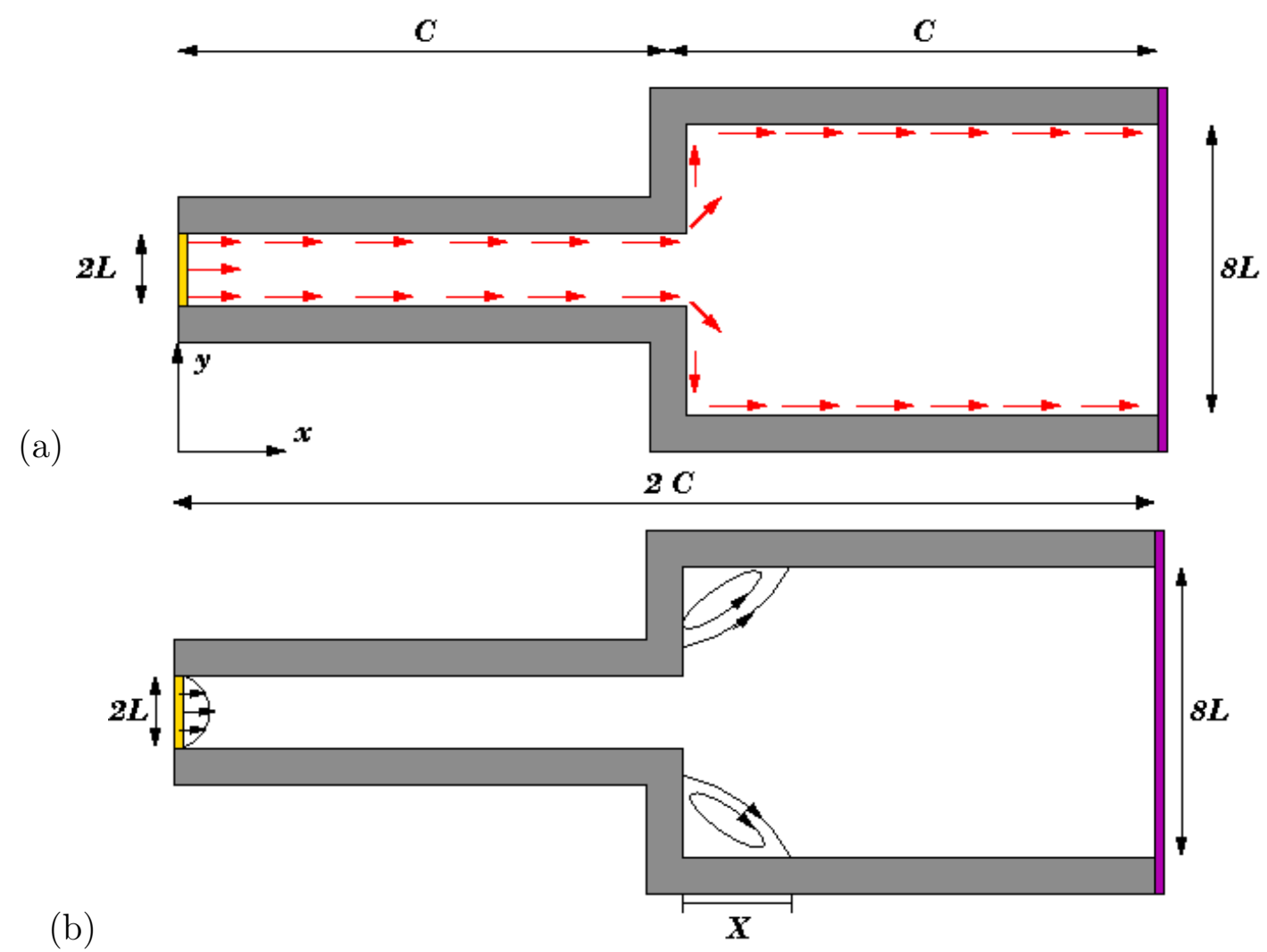

Figura 4.26: Descrição do domínio computacional para a simulação do escoamento em uma expansão planar 1:4. (a) As setas vermelhas representam as condições de contorno utilizadas para o cálculo do ângulo $\phi$ dado pela equação (3.36). (b) $X$ representa o comprimento dos vórtices de canto (Lvortex).

Os parâmetros físicos específicos para o cristal líquido nemático MBBA à $25^{\circ}$ e à $42^{\circ}$ empregados para simular esse problema são apresentados nas Tabelas A.1, A.2 e A.3, respectivamente. Os parâmetros restantes que especificam o escoamento foram:

- Viscosidades: $\eta_{25^{\circ}}=0.0413$ Pa.s e $\eta_{42^{\circ}}=0.0197$ Pa.s;

- Malha: $200 \times 40$ células $(\delta x=\delta y=0.0001) \mathrm{m}$;

- Largura da saída do canal: $8 L=0.004 \mathrm{~m}$;

- Comprimento dos canais: $C=0.01 \mathrm{~m}$;

- Largura da entrada do canal: $2 L=0.001 \mathrm{~m}$;

- Escala de comprimento utilizada: $L=0.0005 \mathrm{~m}$;

- Escalas de velocidade utilizadas: $U_{1}=0.001 \mathrm{~ms}^{-1}$ e $U_{2}=0.002 \mathrm{~ms}^{-1}$; esses valores foram utilizados para calcular a velocidade na entrada do canal por meio da equação (3.85). 
Os números de Reynolds foram calculados utilizando as viscosidades $\eta_{25^{\circ}}$ e $\eta_{42^{\circ}}$ e as escalas de velocidade $U_{1}=0.001 \mathrm{~ms}^{-1}$ e $U_{2}=0.002 \mathrm{~ms}^{-1}$ e obteve-se $R e=0.013$ e $R e=0.026$ para $\eta_{25^{\circ}}$ e, $R e=0.028$ e $R e=0.055$ para $\eta_{42^{\circ}}$, respectivamente. Nos resultados apresentados a seguir, os seguintes números de Ericksen $\left(E r=\frac{U L \eta}{K}\right)$ foram empregados:

\begin{tabular}{c|l|l}
\hline Número de Ericksen & \multicolumn{2}{|l}{ Constantes Elásticas utilizadas para $\eta_{25^{\circ}}$} \\
\hline$E r$ & $R e=0.013$ & $R e=0.026$ \\
\hline 10 & $K=2.065 \times 10^{-9}$ & $K=4.130 \times 10^{-9}$ \\
20 & $K=1.0325 \times 10^{-9}$ & $K=2.065 \times 10^{-9}$ \\
50 & $K=4.130 \times 10^{-10}$ & $K=8.260 \times 10^{-10}$ \\
100 & $K=2.065 \times 10^{-10}$ & $K=4.130 \times 10^{-10}$ \\
200 & $K=1.0325 \times 10^{-10}$ & $K=2.065 \times 10^{-10}$ \\
500 & $K=4.130 \times 10^{-11}$ & $K=8.260 \times 10^{-11}$ \\
1000 & $K=2.065 \times 10^{-11}$ & $K=4.130 \times 10^{-11}$ \\
\hline
\end{tabular}

\begin{tabular}{c|l|l}
\hline Número de Ericksen & \multicolumn{2}{|l}{ Constantes Elásticas utilizadas para $\eta_{42^{\circ}}$} \\
\hline$E r$ & $R e=0.028$ & $R e=0.055$ \\
\hline 10 & $K=9.850 \times 10^{-10}$ & $K=1.970 \times 10^{-9}$ \\
20 & $K=4.925 \times 10^{-10}$ & $K=9.850 \times 10^{-10}$ \\
50 & $K=1.970 \times 10^{-10}$ & $K=3.940 \times 10^{-10}$ \\
100 & $K=9.850 \times 10^{-11}$ & $K=1.970 \times 10^{-10}$ \\
200 & $K=4.925 \times 10^{-11}$ & $K=9.850 \times 10^{-11}$ \\
500 & $K=1.970 \times 10^{-11}$ & $K=3.940 \times 10^{-11}$ \\
1000 & $K=9.850 \times 10^{-12}$ & $K=1.970 \times 10^{-11}$ \\
\hline
\end{tabular}

O objetivo destas simulações é observar os efeitos elásticos e viscosos no escoamento, tal como o surgimento de vórtices de canto e "lip vórtices" no escoamento e, estudar a distribuição do diretor no escoamento. Os resultados obtidos nessas simulações são apresentados e analisados a seguir.

Utilizando os valores dos números de Reynolds e Ericksen apresentados nas Tabelas acima, o código Freeflow2D simulou o problema da expansão até o estado estacionário ser atingido. Para ilustrar que o estado estacionário foi alcançado, as isolinhas das velocidades $u$ e $v$ e da pressão $p$ para $E r=10$ e $R e=0.013$ são mostradas na Figura 4.27, enquanto que a Figura 4.28 mostra a variação da pressão e do gradiente de pressão na linha central do canal, respectivamente. $\mathrm{O}$ perfil das velocidades $u$ e $v$ para $x=10.1$ (meio do canal de entrada), $x=20.1$ (imediatamente após a entrada no canal da expansão) e $x=30.1$ (meio do canal da expansão) são mostradas 
na Figura 4.29. Pode-se observar que na Figura 4.28 que a pressão, com exceção de uma região próxima a entrada da expansão, varia linearmente com $x$ e a Figura 4.29 mostra que a velocidade $v$ é nula na linha central do canal (com exceção de uma região próxima a entrada da expansão) e a velocidade $u$ assume um perfil parabólico no canal de entrada e na expansão. Esses resultados mostram que o estado estacionário foi de fato alcançado. Resultados semelhantes foram obtidos para $E r=10$ e os números de Reynolds $R e=0.026, R e=0.028$ e $R e=0.055$. Resultados similares foram também obtidos para os outros números de Ericksen simulados.
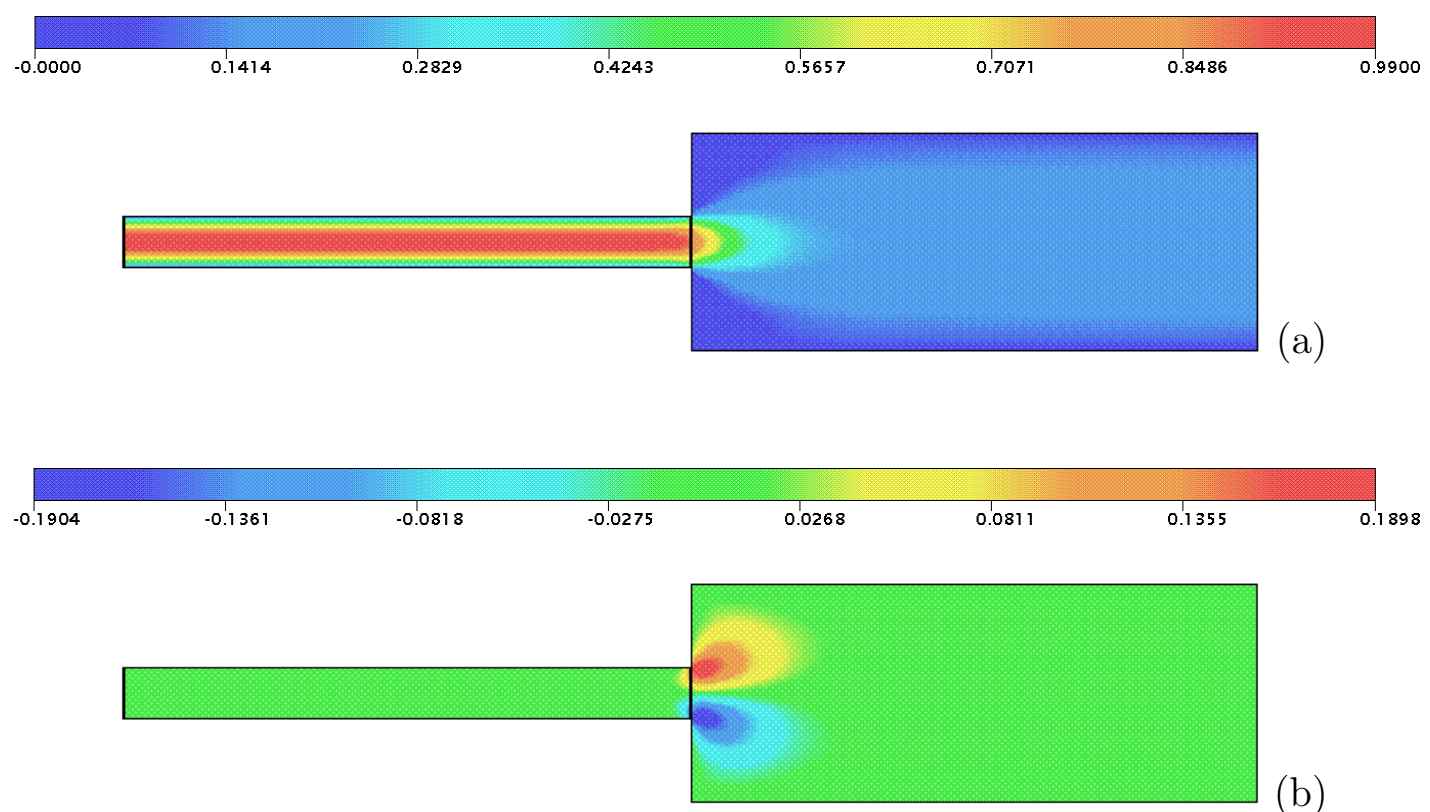

(b)
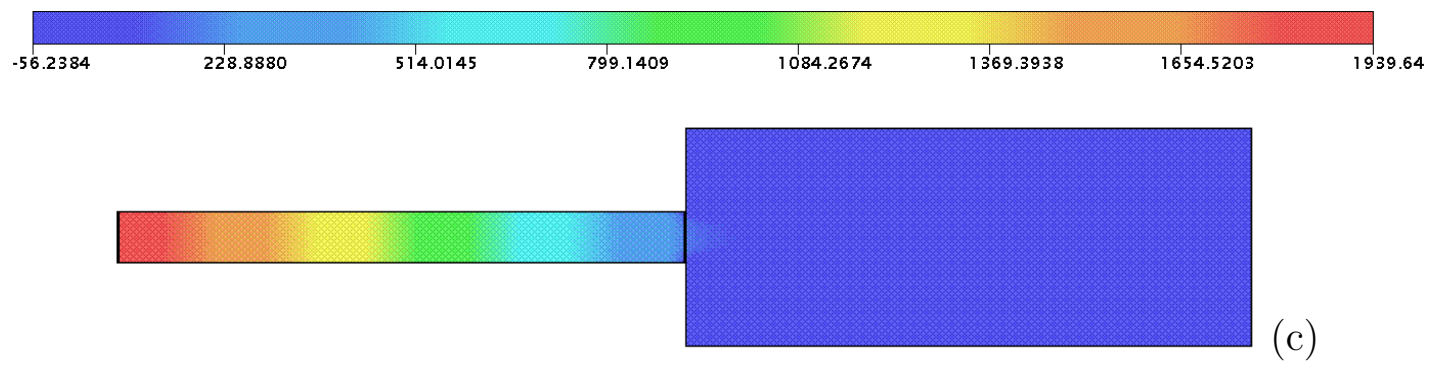

Figura 4.27: Simulação Numérica do escoamento em uma expansão planar 4:1 com $R e=0.013$ e $E r=10$. Isolinhas: (a) velocidade $u$, (b) velocidade $v$, (c) pressão.

Um zoom das soluções obtidas para o diretor próximas a entrada da expansão para $R e=0.013$ e $R e=0.028$ são apresentados nas Figuras 4.30-4.33, respectivamente. A distribuição dos diretores para $R e=0.026$ e $R e=0.055$ foram semelhantes aos obtidos com $R e=0.013$ e $R e=0.028$ e não são mostrados. As respectivas linhas de correntes são mostradas nas Figuras 4.31, 4.32, 4.34 e 4.35. Nas Figuras 4.30-4.33 pode-se observar que os diretores não apresentam grandes diferenças com relação ao número de Reynolds. Porém, a medida 
(a)

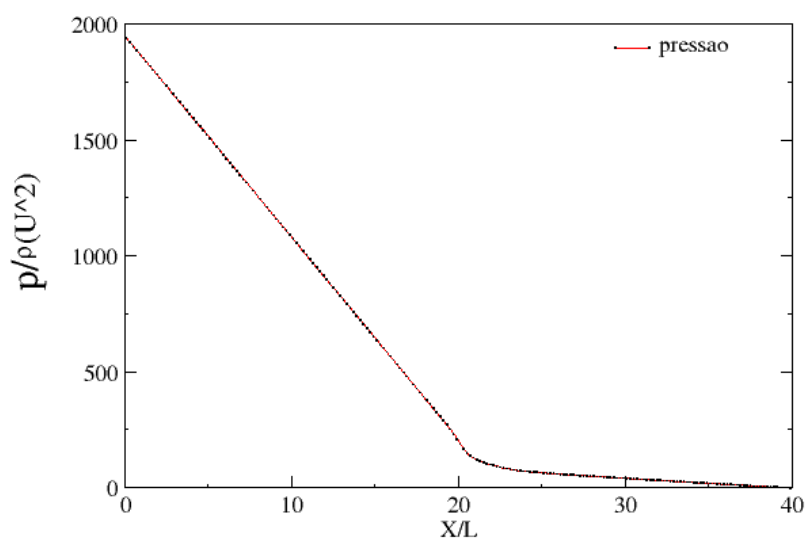

(b)

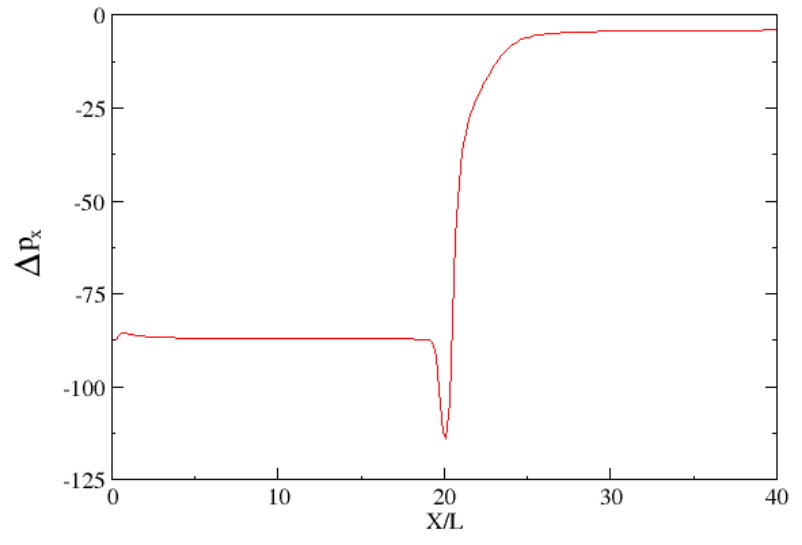

Figura 4.28: Variação da pressão e do gradiente de pressão na linha central do canal com $R e=0.013$ e $E r=10$. (a) pressão, (b) gradiente de pressão.

(a)

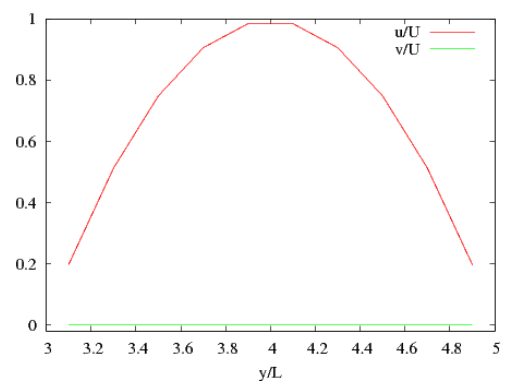

(b)

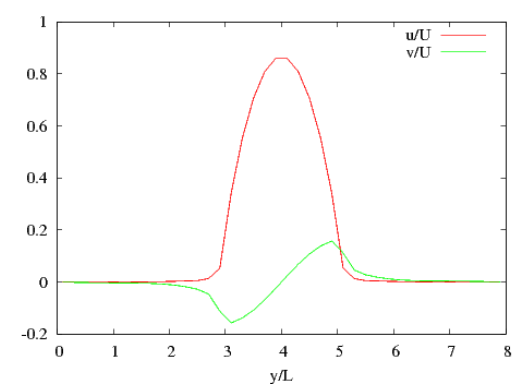

(c)

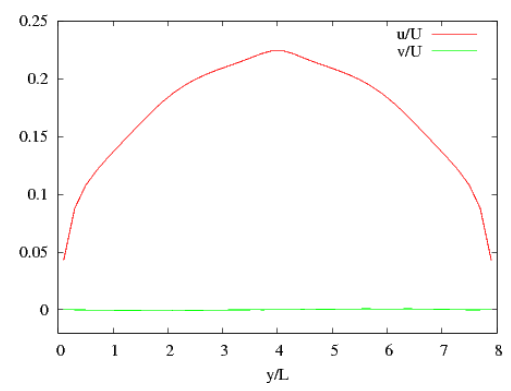

Figura 4.29: Perfis das velocidades $u$ e $v$ em diferentes seções do canal com $R e=0.013$ e $E r=10$. (a) $x=10.1$, (b) $x=20.1$ e (c) $x=30.1$. 
que o número de Ericksen aumenta, observa-se que os diretores aparentam convergir paralelamente a linha central do canal para $R e=0.013$ (também para $R e=0.026$ ) enquanto que para $R e=0.028$ (também para $R e=0.055$ ), os diretores tendem a convergir perpendicularmente a linha central. Esses comportamentos do diretor foram também obtidos por Baleo et al. [10] na simulação de alguns fluidos anisotrópicos.

As Figuras 4.31, 4.32, 4.34 e 4.35 mostram que, para números de Ericksen baixos, as linhas de corrente são suaves e não apresentam nenhum vórtice. Porém, a medida que o número de Ericksen é aumentado, um vórtice no meio da parede da expansão é gerado, o qual se funde com o vórtice que é criado no canto da expansão. Para altos números de Ericksen as linhas de corrente apresentam somente vórtice de canto que aumenta de tamanho com o aumento do número de Ericksen (veja Tabelas 4.2 e 4.3). Pode-se notar nas Tabelas 4.2 e 4.3 e nas Figuras 4.36(a) e 4.36(b), que o aumento do número de Reynolds produz um aumento no tamanho do vórtice de canto. Nas Figuras 4.36(c) e 4.36(d) nota-se que o cristal líquido nemático MBBA à $25^{\circ} \mathrm{C}$ produz vórtices de canto maiores que o nemático $\mathrm{MBBA}$ à $42^{\circ} \mathrm{C}$ quando utiliza-se a mesma escala de velocidade para ambos.

\begin{tabular}{c|l|l}
\hline Número de Ericksen & \multicolumn{2}{|l}{ Comprimento do vórtice de canto. } \\
\hline$E r$ & $R e=0.013$ & $R e=0.026$ \\
\hline 10 & 0.0000 & 0.0000 \\
20 & 0.1000 & 0.1000 \\
50 & 0.2263 & 0.2385 \\
100 & 0.3348 & 0.3687 \\
200 & 0.5953 & 0.6291 \\
500 & 0.7464 & 0.7833 \\
1000 & 0.8929 & 0.9337 \\
\hline
\end{tabular}

Tabela 4.2: Comprimento do vórtice de canto (LVortex) em função do número de Ericksen para o cristal líquido nemático - MBBBA à $25^{\circ}$.

\begin{tabular}{c|l|l}
\hline Número de Ericksen & \multicolumn{2}{|l}{ Comprimento do vórtice de canto. } \\
\hline$E r$ & $R e=0.028$ & $R e=0.055$ \\
\hline 10 & 0.0000 & 0.0000 \\
20 & 0.1000 & 0.1000 \\
50 & 0.2072 & 0.2091 \\
100 & 0.2822 & 0.2906 \\
200 & 0.5073 & 0.5224 \\
500 & 0.6798 & 0.7032 \\
1000 & 0.7509 & 0.7857 \\
\hline
\end{tabular}

Tabela 4.3: Comprimento do vórtice de canto (LVortex) em função do número de Ericksen para o cristal líquido nemático - MBBA à $42^{\circ}$. 

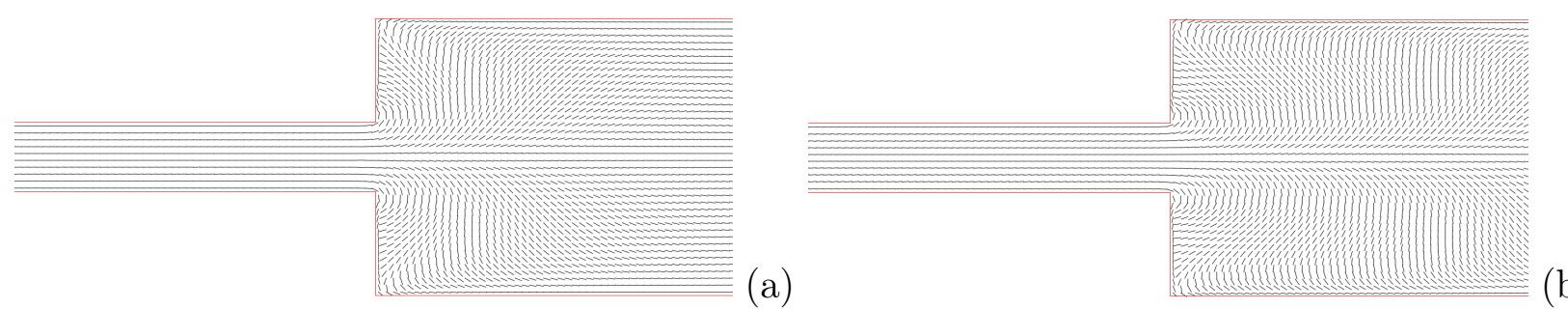

(b)
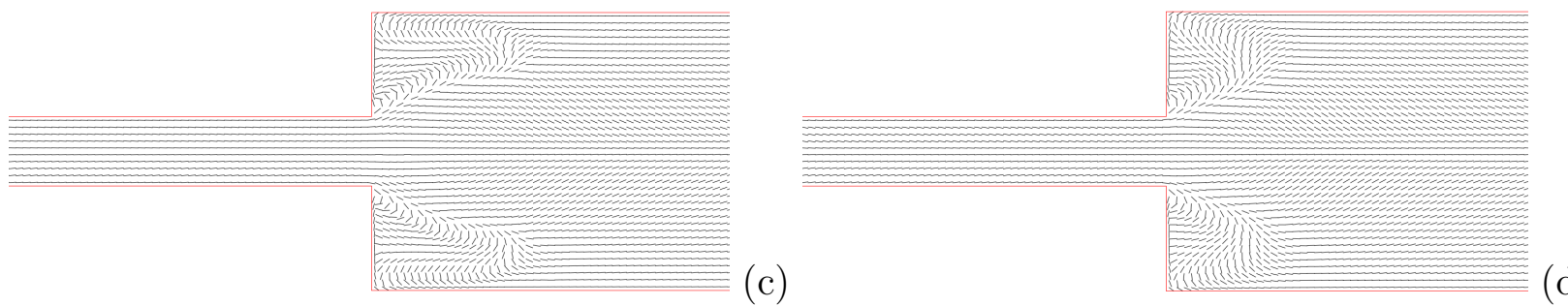

(d)
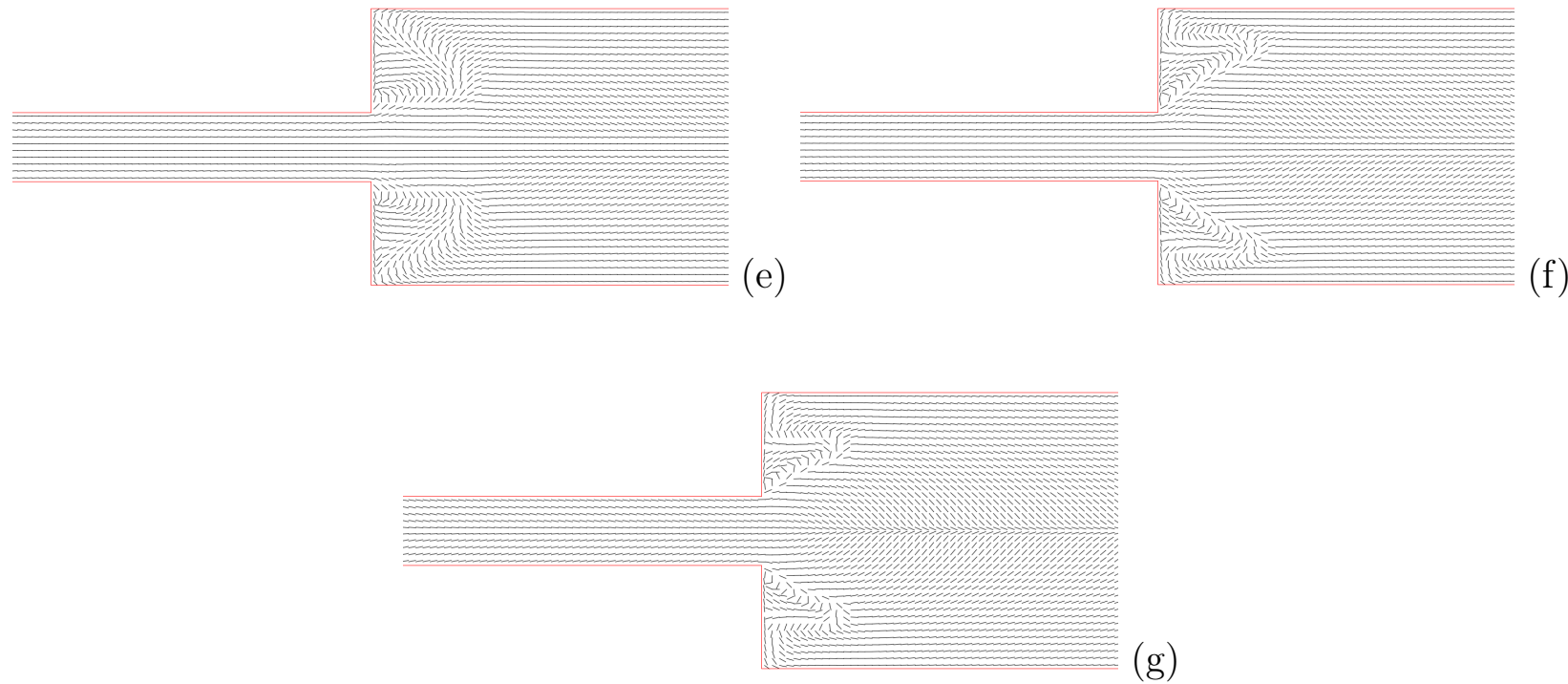

Figura 4.30: Simulação numérica do diretor através de uma expansão planar 4:1 com $R e=$ 0.013. Gráficos do diretor para diferentes números de Ericksen: (a) $E r=10$, (b) $E r=20$, (c) $E r=50,(d) E r=100,($ e) $E r=200,(f) E r=500$ e (g) $E r=1000$. 

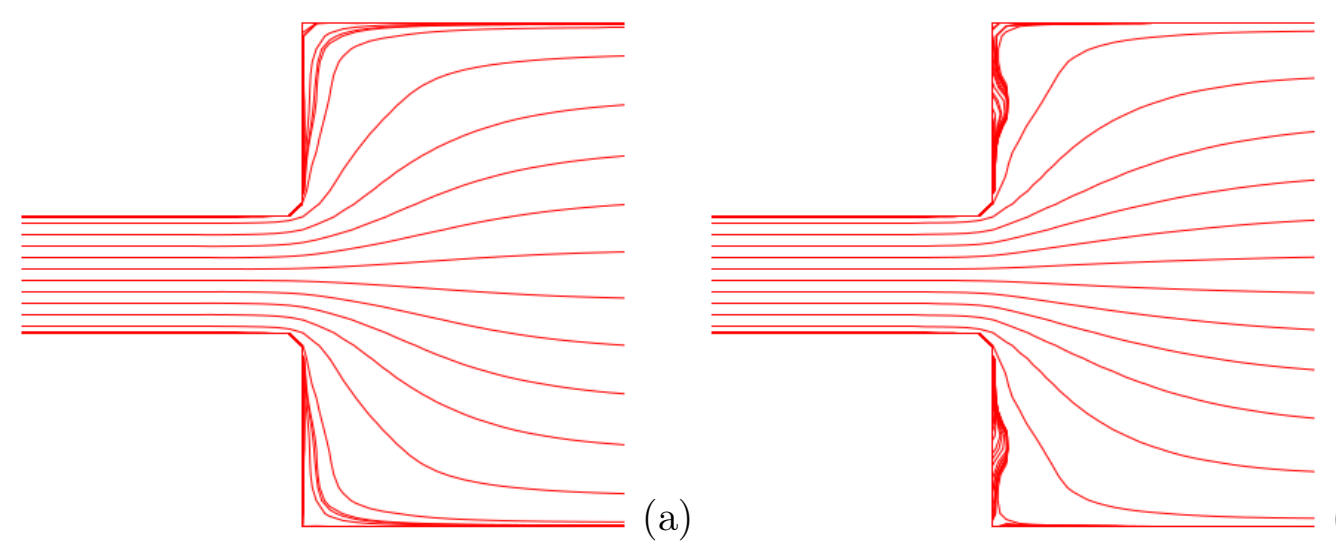

(b)
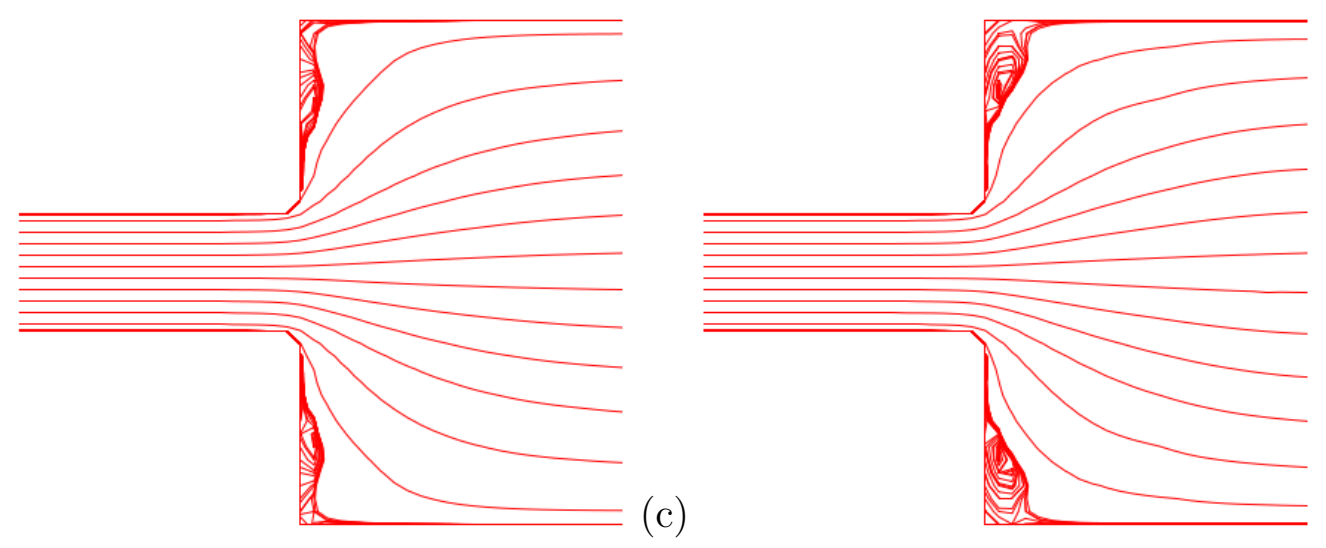

(d)
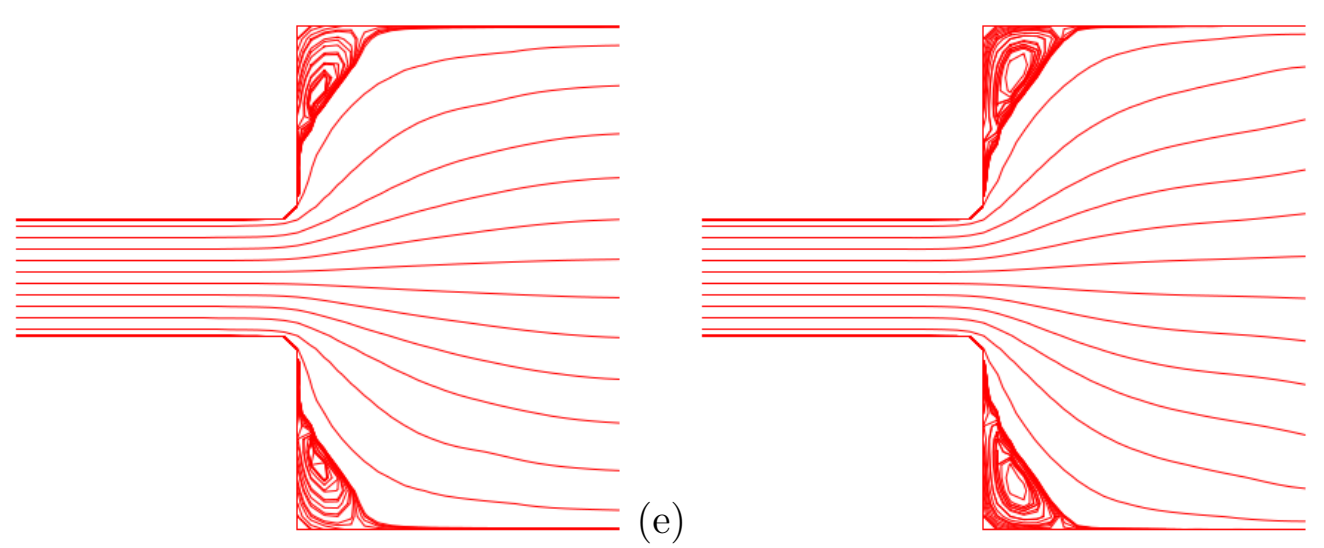

(f)

Figura 4.31: Gráficos das linhas de corrente para $R e=0.013$ e diferentes números de Ericksen: (a) $E r=10$, (b) $E r=50$, (c) $E r=100$, (d) $E r=200$, (e) $E r=500$ e (f) $E r=1000$. 

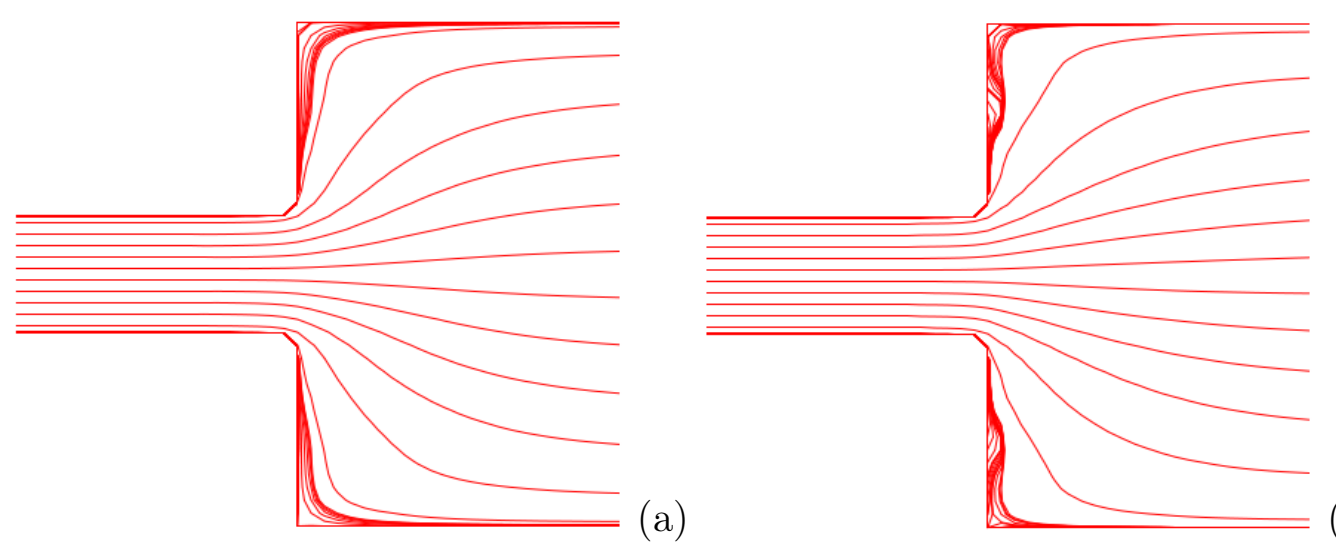

(b)
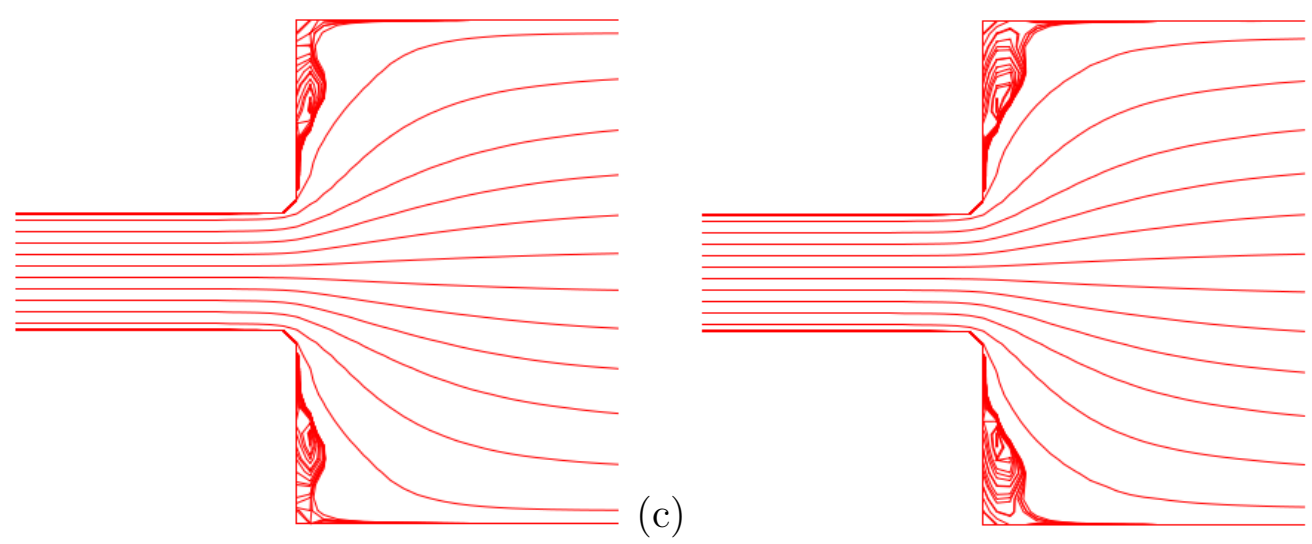

$(d)$
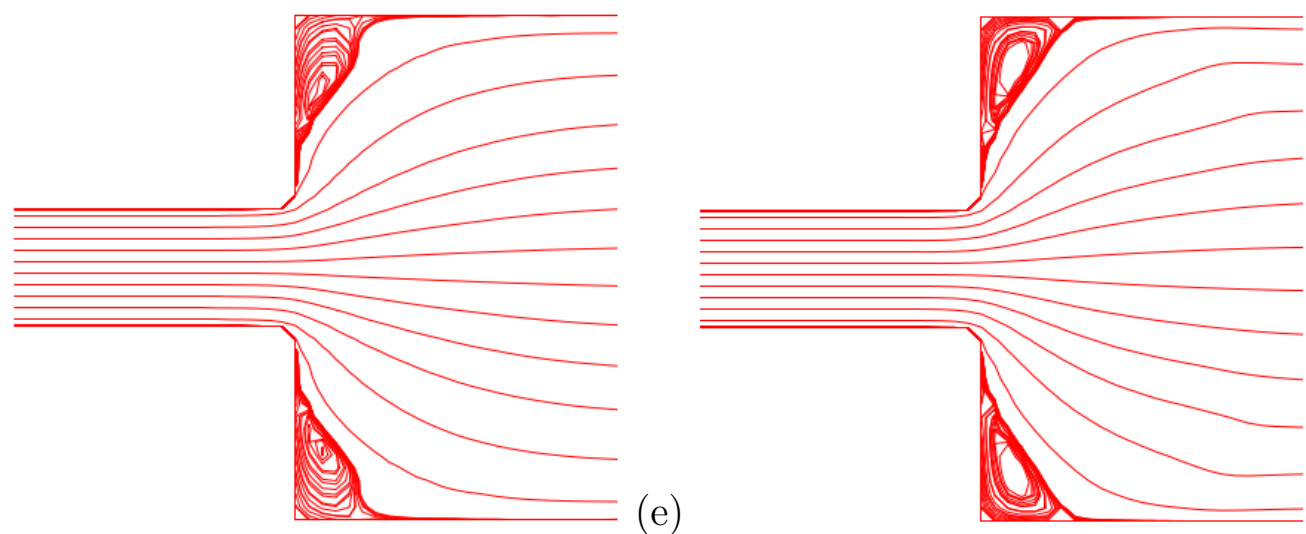

(f)

Figura 4.32: Gráficos das linhas de corrente para $R e=0.026$ e diferentes números de Ericksen: (a) $E r=10$, (b) $E r=50$, (c) $E r=100$, (d) $E r=200$, (e) $E r=500$ e (f) $E r=1000$. 

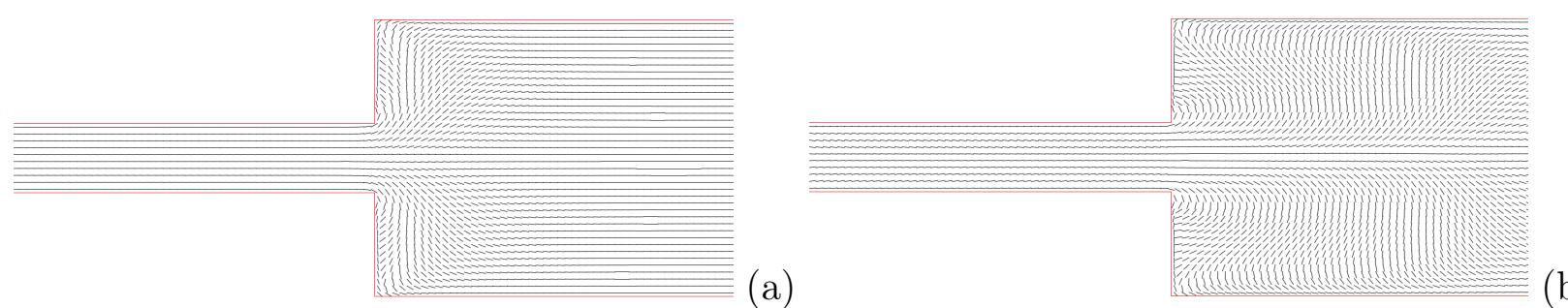

(b)
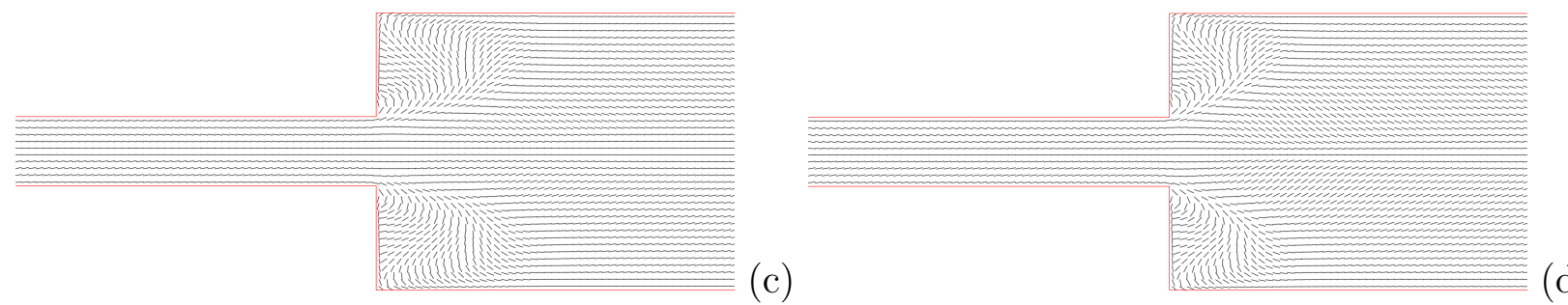

(d)
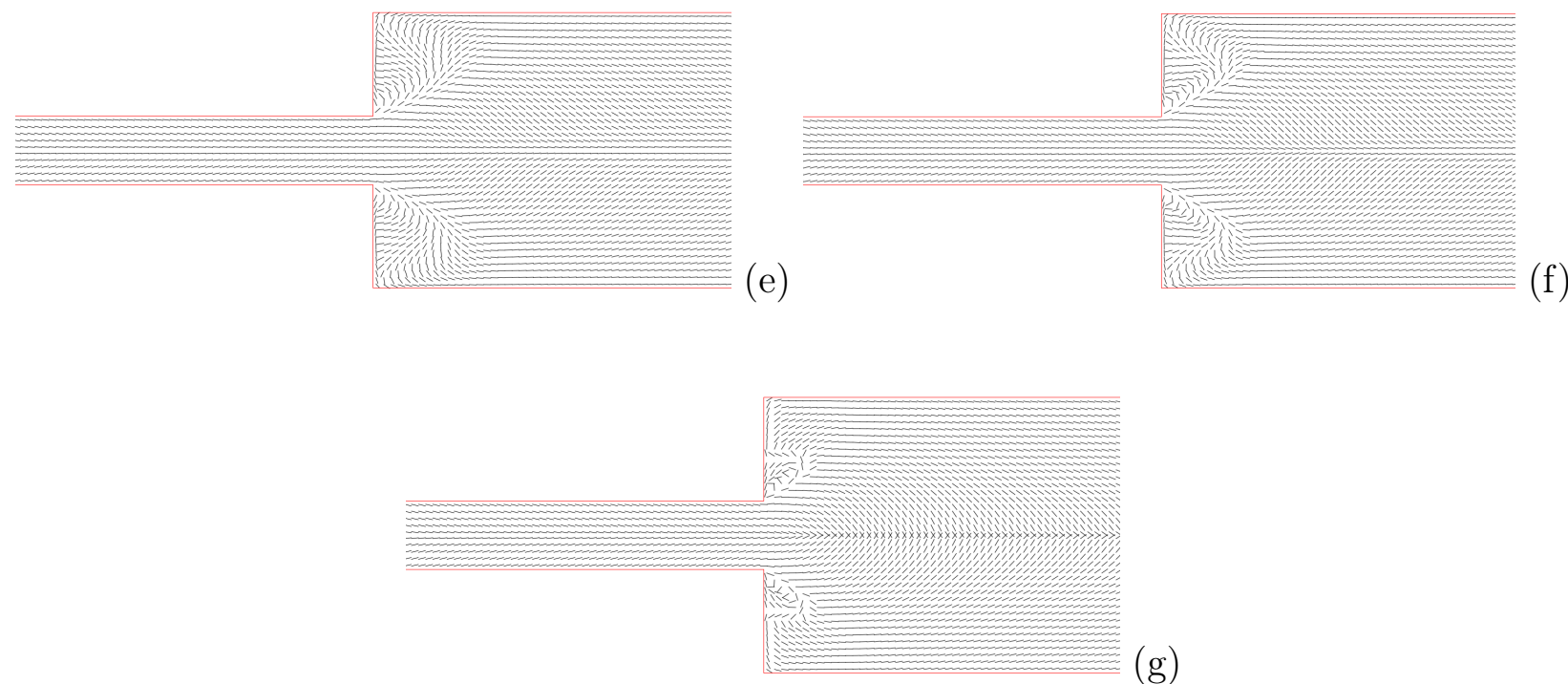

Figura 4.33: Simulação numérica do diretor através de uma expansão planar 4:1 com $R e=$ 0.028. Gráficos do diretor para diferentes números de Ericksen: (a) $E r=10$, (b) $E r=20$ (c) $E r=50,(d) E r=100,($ e) $E r=200$, (f) $E r=500$ e (g) $E r=1000$. 

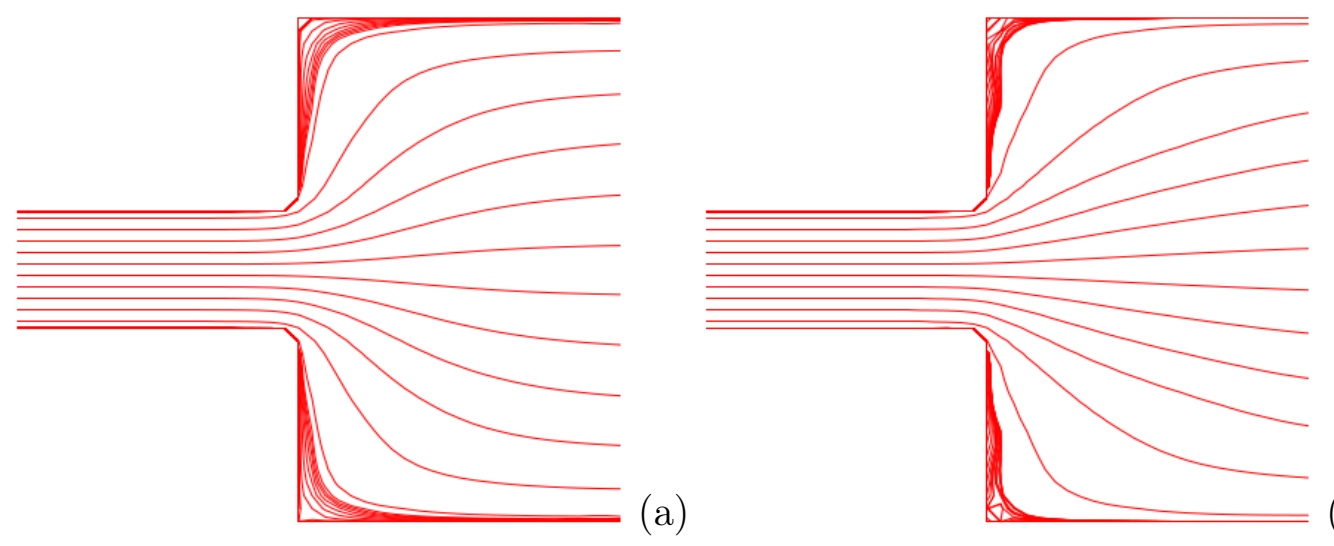

(b)
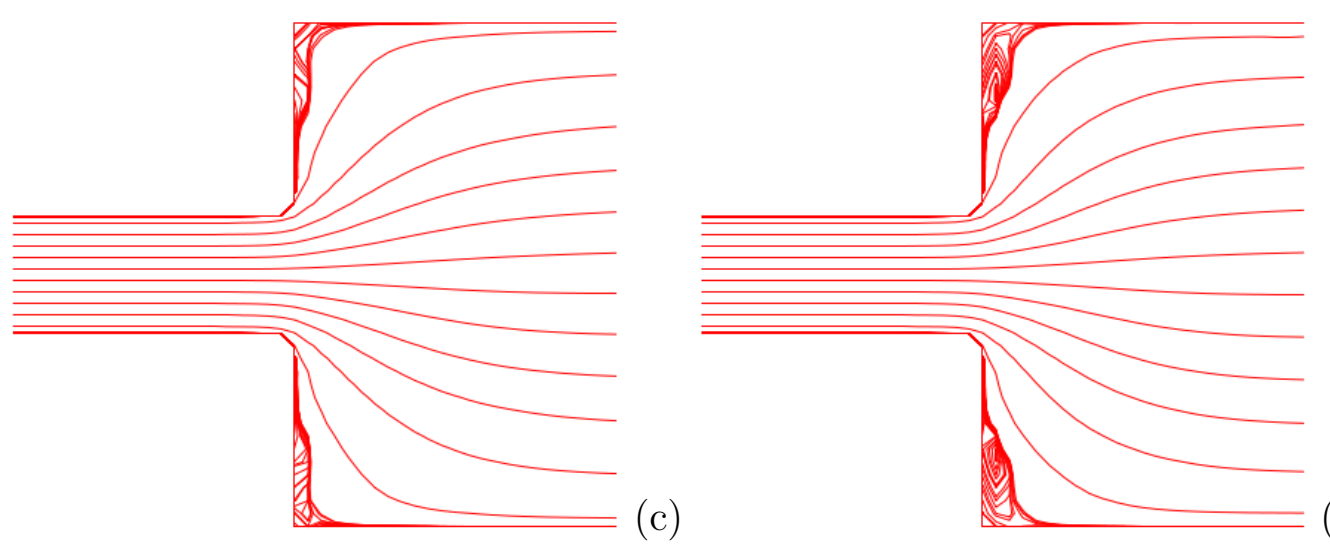

(d)
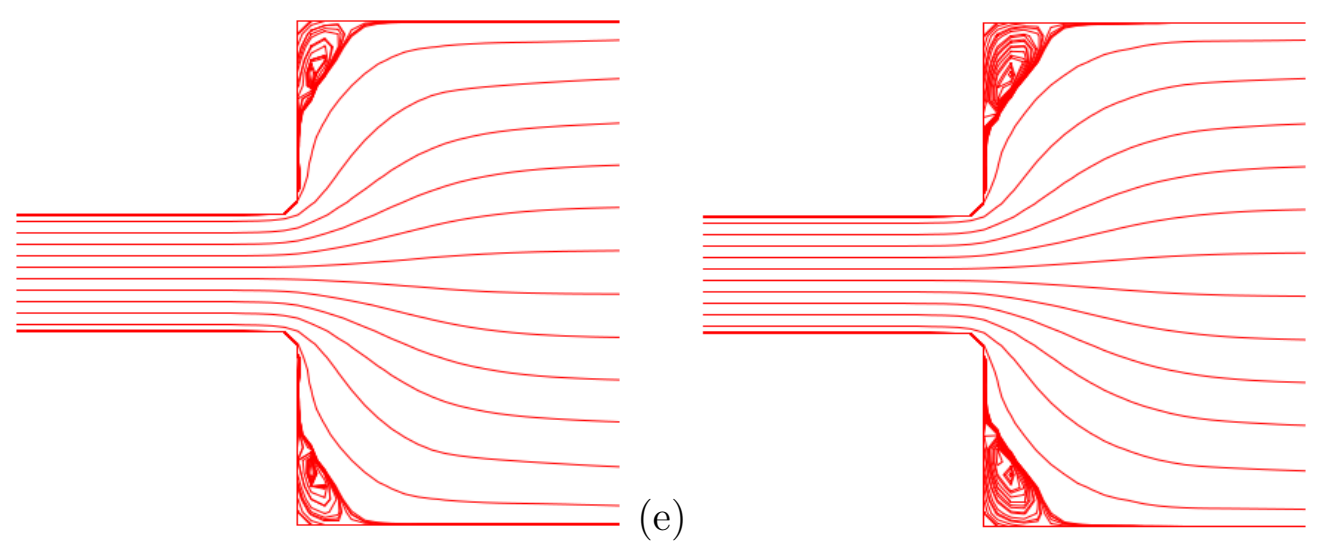

(f)

Figura 4.34: Gráficos das linhas de corrente para $R e=0.028$ e diferentes números de Ericksen: (a) $E r=10$, (b) $E r=50$, (c) $E r=100$, (d) $E r=200$, (e) $E r=500$ e (f) $E r=1000$. 

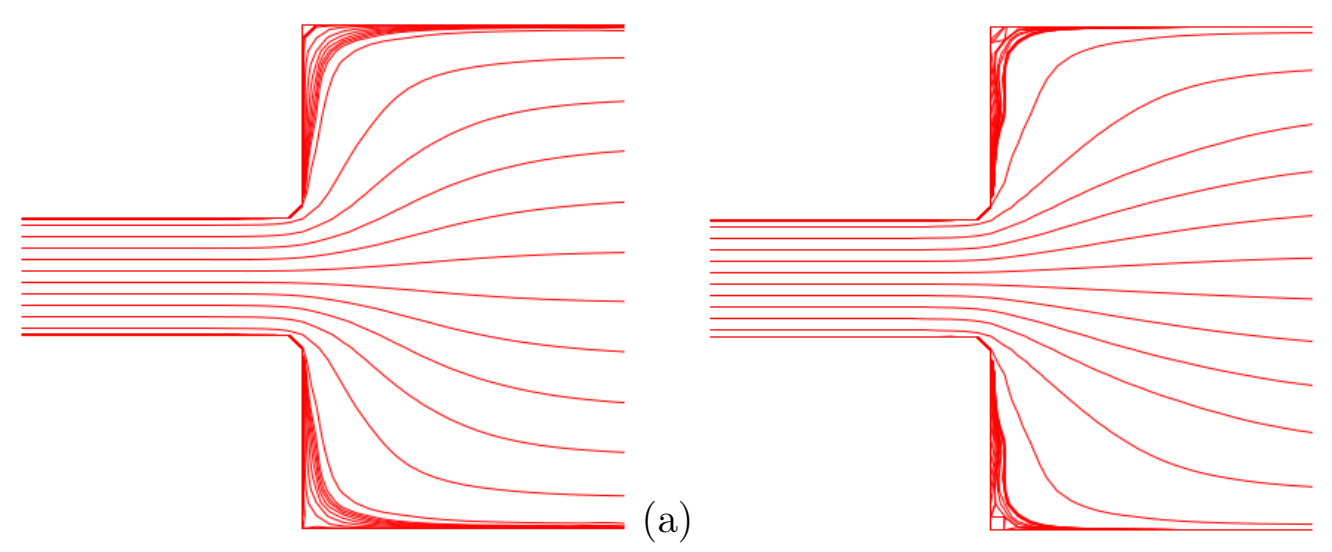

(b)
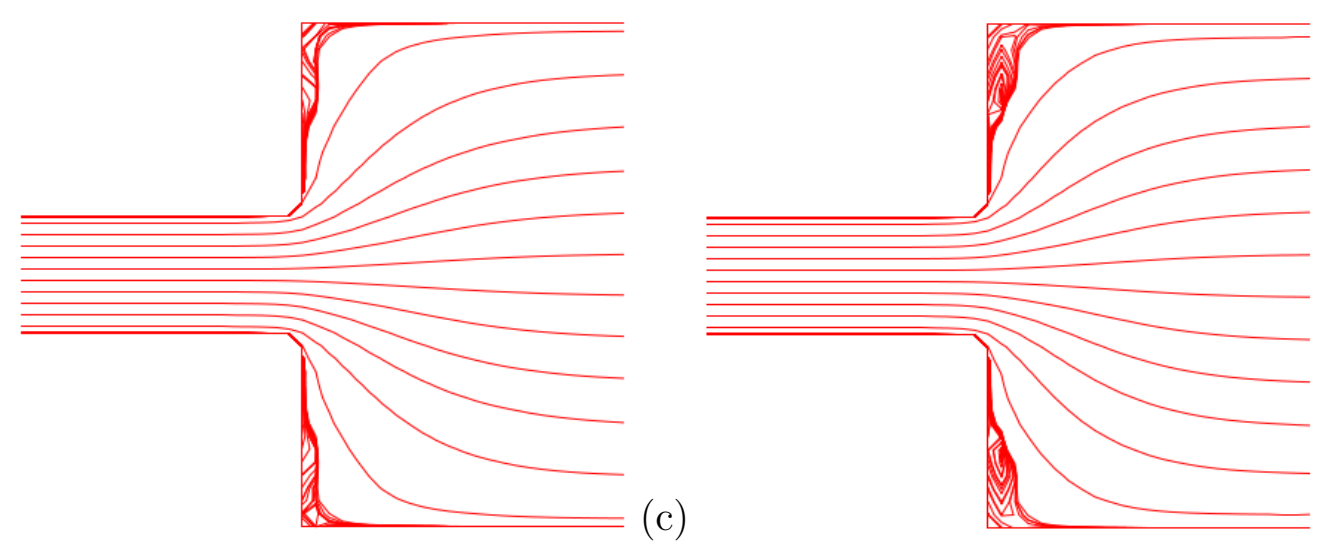

(d)
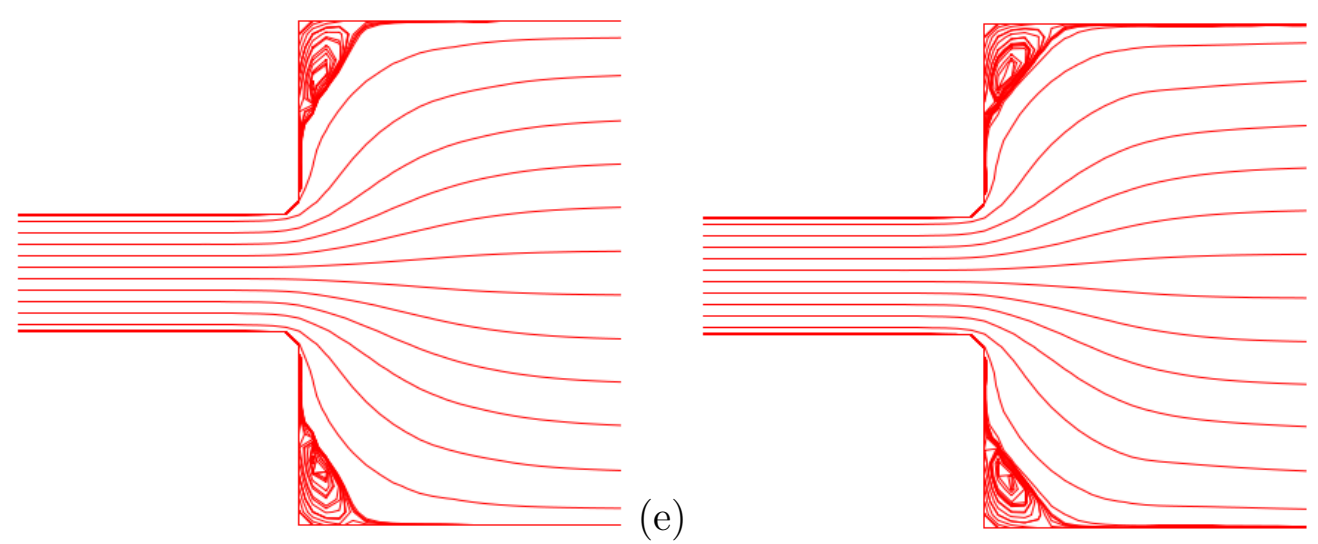

(f)

Figura 4.35: Gráficos das linhas de corrente para $R e=0.055$ e diferentes números de Ericksen: (a) $E r=10$, (b) $E r=50$, (c) $E r=100$, (d) $E r=200$, (e) $E r=500$ e (f) $E r=1000$. 

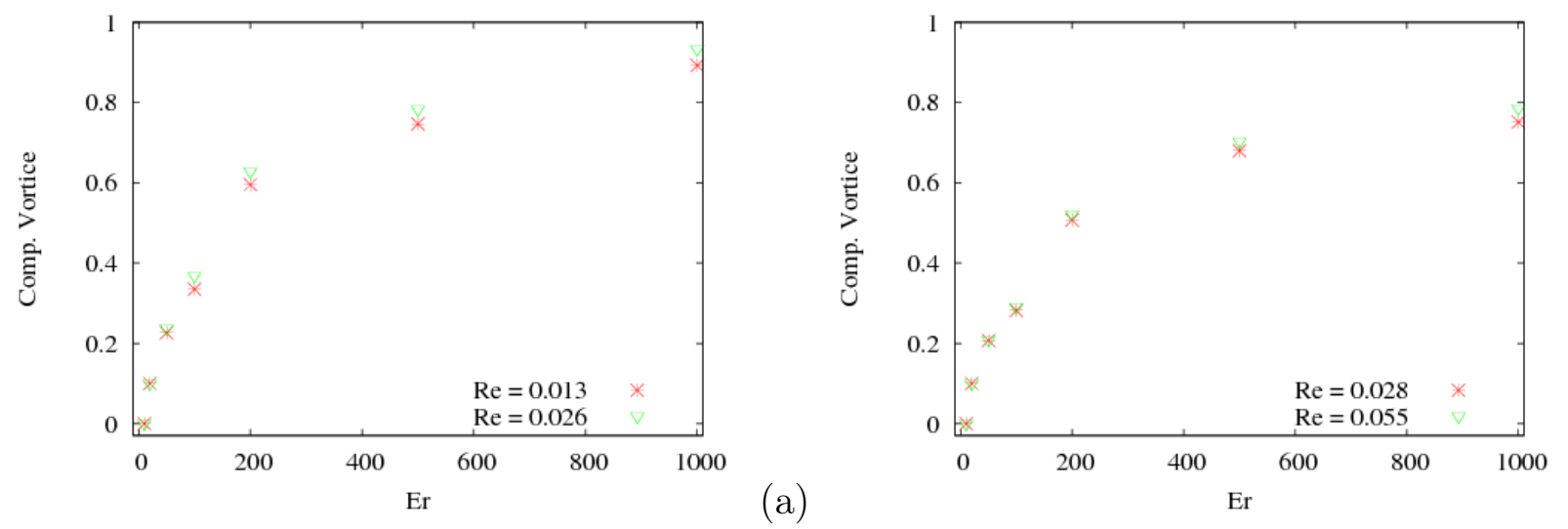

(b)

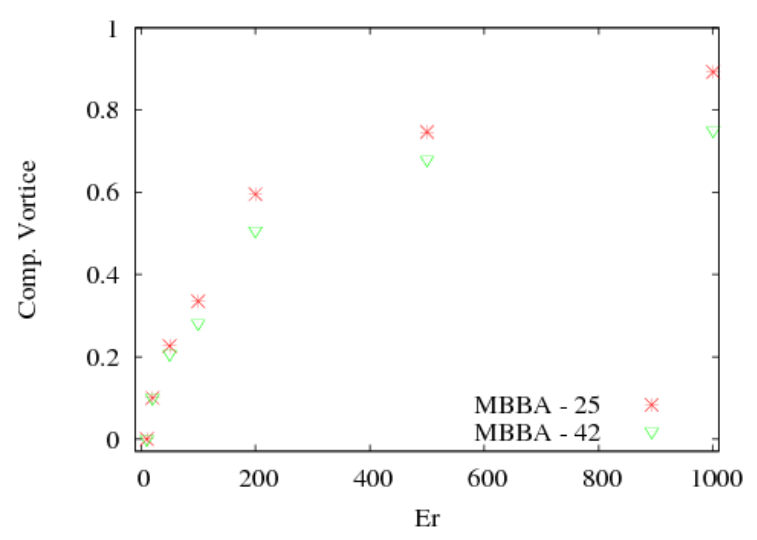

(c)

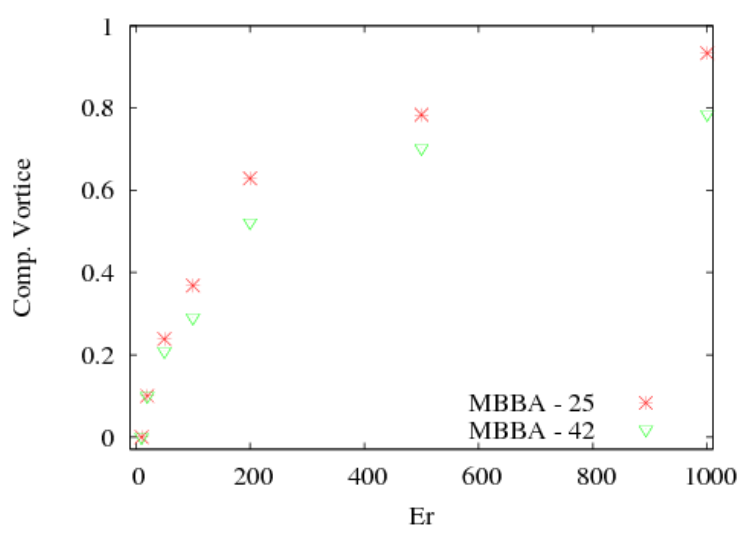

(d)

Figura 4.36: Gráficos da variação do comprimento do vórtice em função do número de Ericksen: (a) cristal líquido nemático MBBA à $25^{\circ} \mathrm{C}$, (b) cristal líquido nemático MBBA à $42^{\circ} \mathrm{C}$, (c) escala de velocidade utilizada $U_{1}=0.001 \mathrm{~ms}^{-1}$ e (d) escala de velocidade utilizada $U_{2}=0.002 \mathrm{~ms}^{-1}$. 


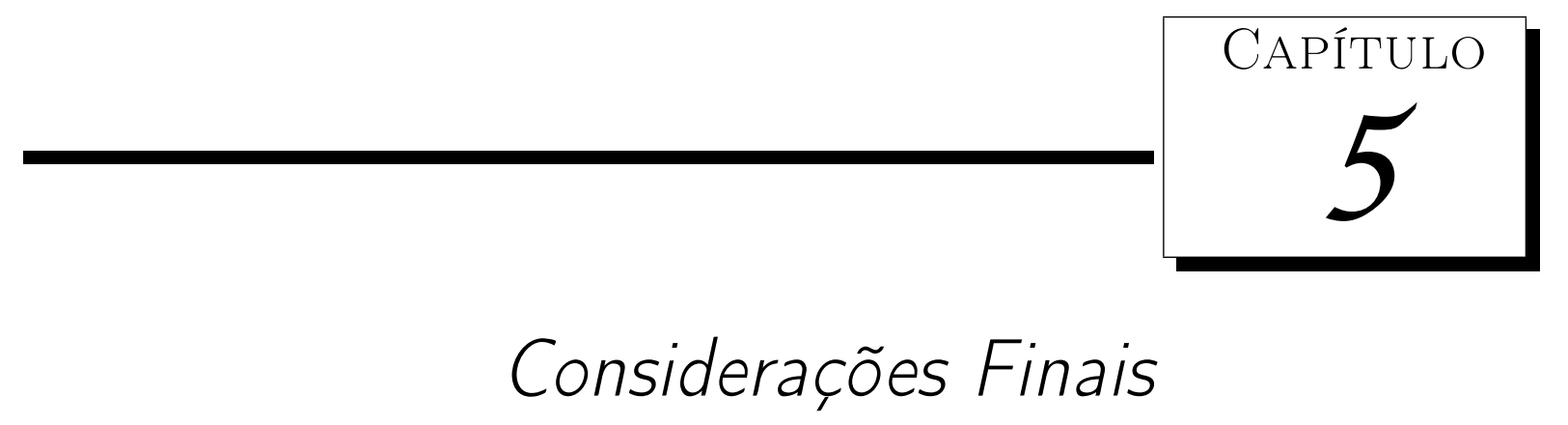

Esta tese apresentou os desenvolvimentos realizados para a obtenção de métodos numéricos para a simulação de escoamentos de cristais líquidos nemáticos governados pelas equações dinâmicas de Ericksen-Leslie. O trabalho desenvolvido pode ser dividido em três tópicos principais: conceitos introdutórios da teoria dos cristais líquidos, desenvolvimento de um método numérico para simular escoamentos tridimensionais de cristais líquidos nemáticos sob forte campo magnético e desenvolvimento de um método numérico para a simulação de escoamentos bidimensionais de cristais líquidos nemáticos.

No Capítulo 1, foi realizada uma revisão sobre cristais líquidos. Foram apresentadas os conceitos da teoria de cristais líquidos, uma descrição detalhada das aplicações dos cristais líquidos, as equações dinâmicas de Ericksen-Leslie, bem como uma revisão sobre o método GENSMAC e ainda uma revisão sobre escoamentos de cristais líquidos nemáticos.

Os outros tópicos foram apresentados nos Capítulos 2, 3 e 4. Inicialmente, no Capítulo 2, foi desenvolvido um método numérico de diferenças finitas para simular escoamentos tridimensionais de cristais líquidos nemáticos sob efeito de um forte campo magnético, governados pelas equações dinâmicas de Ericksen-Leslie. Como sugerido por Leslie [83], se o campo magnético for suficientemente forte então o diretor permanece fixo no escoamento e as equações de conservação de momento angular (1.5) podem ser desprezadas. Assim, foram apresentadas as equações básicas (1.2) e (1.4) para escoamentos incompressíveis tridimensionais, isotérmicos de cristais líquidos nemáticos sob forte campo magnético e suas respectivas formas adimensionais. As equações governantes foram resolvidas empregando-se uma metodologia baseada no algoritmo GENSMAC3D. O método numérico descrito no Capítulo 2 foi implementado no sistema Freeflow3D o qual foi utilizado para simular o escoamento de um cristal líquido nemático sob forte campo magnético em um canal tridimensional. Para validar a técnica numérica desenvol- 
vida, o escoamento entre duas placas paralelas foi simulado até que o estado estacionário fosse atingido e os resultados numéricos foram comparados com a solução analítica (apresentada por Stewart [123]) para escoamentos totalmente desenvolvidos entre duas placas paralelas. Os resultados mostraram uma boa concordância entre as soluções numérica e analítica e o erro relativo entre as soluções, calculado em três diferentes malhas, demonstrou a convergência do método numérico desenvolvido no Capítulo 2.

No Capítulo 3, foi apresentado um método numérico de diferenças finitas para a simulação de escoamentos bidimensionais de cristais líquidos nemáticos. Para o desenvolvimento desta técnica numérica não foi considerado a hipótese de um forte campo magnético e desta forma, as equações de conservação de momento angular não foram desprezadas. As equações governantes em duas dimensões para escoamentos incompressíveis de cristais líquidos nemáticos e suas respectivas formas adimensionais são apresentadas e foram resolvidas empregando-se uma metodologia baseada no algoritmo GENSMAC. Para a validação da técnica numérica desenvolvida, foi considerado um escoamento em um canal bidimensional. Uma solução analítica para o escoamento totalmente desenvolvido em um canal bidimensional foi encontrada. Utilizando a hipótese de que não existe torque externo atuando no escoamento (ver Carou et. al. $[19,20,21])$, pode-se mostrar que o ângulo do diretor é pequeno. Nessas condições, o sistema de equações resultantes foi resolvido analiticamente e foram obtidas expressões para a velocidade $u$, para o ângulo de orientação do diretor $\phi$ e consequentemente, foram obtidas expressões analíticas para as componentes do tensor não-newtoniano $\Phi_{i j}$. O escoamento desenvolvido em um canal foi simulado usando refinamento de malha. Os resultados obtidos mostraram uma boa concordância entre as soluções numéricas e analíticas e o erro relativo entre as soluções, calculado em três diferentes malhas, demonstrou a convergência do método numérico.

No Capítulo 4, a técnica numérica desenvolvida no Capítulo 3 foi utilizada para a simulação de escoamentos bidimensionais de cristais líquidos nemáticos. Os escoamentos de um cristal líquido nemático em geometrias complexas, tais como L-canal, contração planar 4:1 e expansão planar 1:4 foram considerados. Com relação a simulação numérica em L-canais, primeiramente, o efeito das condições de contorno no escoamento foi investigado. Foi observado que as condições de contorno aplicadas ao ângulo $\phi$ produziram diferentes efeitos no campo dos diretores. As várias condições de contorno aplicadas ao ângulo do diretor não produziram maiores mudanças nas linhas de corrente da velocidade no L-canal, esse comportamento foi semelhante aos resultados observados por Chono et. al. [24] para certos fluidos viscoelásticos. Ainda com relação a simulação em L-canais, foi realizado um estudo do efeito do número de Ericksen e da mudança de geometria do canal no escoamento. As condições de contorno utilizadas foram aquelas apresentadas na Figura 4.1(a). Foi observado que as condições de contorno impostas para o ângulo $\phi$ foram obedecidas para os valores de $E r$ utilizados, enquanto que a mudança de geometria ocasionou o surgimento de um "lip vortex" na saída do canal de entrada. O número de Reynolds foi mantido fixo e o número de Ericksen foi variado. Nesta situação, observou-se 
que quando o número de Ericksen foi aumentado, o tamanho desse "lip vortex" diminuiu, o qual não pode ser observado para $E r=250$ e, portanto acredita-se que o aparecimento do "lip vortex" foi devido a mudança de geometria do L-canal e, a diminuição da elasticidade (ocasionado pelo aumento de $E r$ ) foi o causador da atenuação do "lip vortex".

Em relação a simulação numérica do escoamento através de uma contração foram investigados o comportamento dos diretores e o comportamento dos vórtices de canto no escoamento. O comportamento dos vórtices pode ser afetado pelos números de Reynolds e de Ericksen, o tipo de contração e pelas propriedades reológicas de cada material (viscosidade e elasticidade). Observou-se que, para $R e=0.013$, os diretores concordaram com as condições de contorno impostas. Os resultados obtidos para os diretores para $R e=0.026$ e $R e=0.066$ foram semelhantes aos resultados obtidos com $R e=0.013$. Quanto aos vórtices de canto, observou-se uma diminuição no tamanho dos mesmos à medida que o número de Ericksen aumentou. Esses resultados foram apresentados na Tabela 4.1 e ilustrados na Figura 4.25 e, à partir desses resultados acredita-se que a diminuição do vórtice de canto foi devido aos efeitos elásticos no escoamento. Os resultados obtidos na simulação numérica do escoamento em uma expansão 1:4 mostraram o aparecimento de vórtices de canto e "lip vortex". Acredita-se que o aparecimento desses vórtices são devidos aos efeitos elásticos e viscosos no escoamento. Os resultados obtidos para os diretores não apresentaram grandes variações com relação a variação do número de Reynolds. Porém, à medida que o número de Ericksen aumentou, observou-se que os diretores deixaram de se posicionar paralelamente a linha central do canal e passaram a convergir perpendicularmente a linha central do canal (fato observado para $E r=500$ e 1000). Esses comportamentos foram observados por Baleo et. al. [10] na simulação de alguns fluidos anisotrópicos. Quanto a questão do surgimento de vórtices, os resultados obtidos mostraram que para números de Ericksen baixos não ocorreu o aparecimento de vórtices. Porém, quando se aumentou o número de Ericksen, foi gerado um vórtice no meio da parede da expansão "lip vortex", o qual se fundiu com o vórtice criado no canto da expansão. Para números de Ericksen altos, foram obtidos apenas vórtices de canto que aumentaram de tamanho a medida que o número de Ericksen foi aumentado. Esses resultados são apresentados nas Tabelas 4.2 e 4.3 .

Como proposta de trabalho futuro, pretende-se estender o método numérico desenvolvido nesta tese para simular escoamentos de cristais líquidos poliméricos (LCPs) utilizando a teoria desenvolvida por Doi e Edwards [41]. Essa teoria tem sido utilizada por vários investigadores (ver por exemplo [56, 55, 144, 145]) mas a aplicação dessa teoria a escoamentos em duas dimensões utilizando as equações completas de Doi e Edwards ainda não foi considerada. Mais detalhes sobre a teoria de Doi e Edwards [41] podem ser encontrados em [111, 112]. Pretende-se desenvolver um método numérico para resolver as equações resultantes e desenvolver um código computacional para simular escoamentos em duas dimensões descritos pela teoria de Doi e Edwards [41]. Pretende-se ainda estudar as equações governantes para cristais líquidos esméticos e formular e implementar um método numérico para simular escoamentos de cristais líquidos 
esméticos. Detalhes mais avançados da teoria e aplicações dos cristais líquidos esméticos podem ser encontrados no livro recente de Stewart [123], assim como nos artigos [122, 139]. 


\section{Referências Bibliográficas}

[1] M. Alves, P. Oliveira, and F. Pinho. A convergent and universally bounded interpolation scheme for the treatment of advection. International Journal for Numerical Methods in Fluids, 41:47-75, 2003.

[2] M. Ben Amar and L. J. Cummings. Fingering instabilities in driven thin nematic films. Physics of Fluids, 13:1160-1166, 2001.

[3] M. Ambrozic, P. Formoso, A. Golemme, and S. Žumer. Anchoring and droplet deformation in polymer disperse liquid crystals: Nmr study in an electric field. Physics Review E, 56:1825-1832, 1997.

[4] A. A. Amsden and F. H. Harlow. A simplified mac technique for incompressible fluid flow calculations. Journal of Computational Physics, 6:332-335, 1970.

[5] A. Anzelius. Über die bewegung der anisotropen flüssigketen. Uppsala Univ. Arsskr., Mat. och Naturvet., 1:1-84, 1931.

[6] M. S. B. Araujo. Extensão de GENSMAC para escoamentos de fluidos governados pelos modelos integrais Maxwell e K-BKZ. PhD thesis, Universidade de São Paulo, Instituto de Ciências Matemáticas e Computação, São Carlos, 2006.

[7] R. Aris. Vectors, Tensors and the Basic Equations of Fluid Mechanics. Dover, New York, 1989.

[8] R. J. Atkin. Poiseuille flow of liquid crystals of the nematic type. Archieves of Rational Mechanics and Analysis, 38:224-240, 1970.

[9] C. Atkinson and P. J. S. Pereira. Flow of a nematic liquid crystal near the leading edge of an infinite prism. The Quarterly Journal of Mechanics and Applied Mathematics, 58:503-533, 2005. 
[10] J. N. Baleo, M. Vincent, P. Navard, and Y. Demay. Finite element simulation of flow and director orientation of viscous anisotropic fluids in complex $2 \mathrm{~d}$ geometries. Journal of Rheology, 36:663-702, 1992.

[11] V. V. Belyaev. Relationship between nematic viscosities and molecular structure, section 8.4 in reference [43].

[12] L. M. Blinov. Electro-optical and Magneto-optical Properties of Liquid Crystals. Wiley, Chichester, 1983.

[13] D. Boger, D. Hur, and R. Binnington. Further observations of elastic effects in tubular entry flows. Journal of Non-Newtonian Fluid Mechanics, 20:31-49, 1986.

[14] G. H. Brown and W. G. Shaw. The mesomorphic state - liquid crystals. Chemical Reviews, 57:1049-1157, 1957.

[15] G. H. Brown and J. J. Wolken. Liquid Crystals and Biological Structures. Academic Press, New York, 1979.

[16] J. D. Bunning, D. A. Crellin, and T. E. Faber. The effect of molecular biaxiality on the bulk properties of some nematic liquid crystals. Liquid Crystals, 1:37-51, 1986.

[17] W. R. Burghardt and G. G. Fuller. Transient shear flow of nematic liquid crystals: manifestations of director tumbling. Journal of Rheology, 34:959-992, 1990.

[18] M. C. Calderer and C. Liu. Poiseuill flow of nematic liquid crystals. International Journal of Engineering Science, 38:1007-1022, 2000.

[19] J. Quintans Carou, B. R. Duffy, N. J. Mottram, and S. K. Wilson. Steady flow of a nematic liquid crystal in a slowly varying channel. Molecular Crystals and Liquid Crystals, 438:1801, 2005.

[20] J. Quintans Carou, B. R. Duffy, N. J. Mottram, and S. K. Wilson. Shear-driven and pressure-driven flow of a nematic liquid crystal in a slowly varying channel. Physics of Fluids, 18:027105-1-027105-13, 2006.

[21] J. Quintans Carou, N. J. Mottram, S. K. Wilson, and B. R. Duffy. A mathematical model for blade coating of a nematic liquid crystal. Liquid Crystals, 34:621-631, 2007.

[22] A. Castelo, M. F. Tomé, J. A. Cuminato, and S. McKee. Freeflow: An integrated simulation system for three-dimensional free surface flows. Computing and Visualization in Science, 2:199-210, 2000.

[23] S. Chandrasekhar. Liquid Crystals, 2nd Edition. Cambridge University Press, 1992. 
[24] S. Chono and Y. Iemoto. Numerical simulation of viscoelastic flow in two-dimensional l-shaped channels. Journal of Rheology, 36:335-356, 1992.

[25] S. Chono and T. Tsuji. Numerical simulation of nematic liquid crystalline flows around a circular cylinder. Molecular Crystals and Liquid Crystals, 309:217-236, 1998.

[26] S. Chono, T. Tsuji, and M. M. Denn. Spatial development of director orientation of tumbling nematic liquid crystals in pressure-driven channel flow. Journal of Non-Newtonian Fluid Mechanics., 79:515-527, 1998.

[27] A. J. Chorin. A numerical method for solving incompressible vsicous flow problems. Journal of Computational Physics, 2:12-26, 1967.

[28] A. J. Chorin. Numerical solution of the navier-stokes equations. Mathematics of Computation, 2:745-762, 1968.

[29] P.J. Collings. Liquid crystals: nature's delicate phase of matter. Adam Hilger, 1990.

[30] P.J. Collings and M. Hird. Introduction to Liquid Crystals. Taylor and Francis, 1997.

[31] R. Courant, K. Friedrichs, and H. Lewy. On the partial differential equations of mathematical physics. IBM Journal Research and Development, 11:215-234, 1967.

[32] P. A. Cruz, M. F. Tomé, I. W. Stewart, and S. McKee. A numerical method for solving the dynamic three-dimensional ericksen-leslie equations for nematic liquid crystals subject to a strong magnetic field. Journal of Non-Newtonian Fluid Mechanics, 165:113-157, 2010.

[33] P. A. Cruz, M. F. Tomé, I. W. Stewart, and S. McKee. Numerical investigation of director orientation and flow of nematic liquid crystal in a planar 1:4 expansion. Journal of Mechanics of Materials and Strucutres, —:em impressão, 2011.

[34] P. A. Cruz, M. F. Tomé, I. W. Stewart, and S. McKee. Numerical solution of the ericksen-leslie dynamic equations for two-dimensional nematic liquid crystl flows. Journal of Computational Physics, —:em submissão, 2011.

[35] P. K. Currie. Approximate solutions for poiseuille flow of liquid crystals. Rheologica Acta, 14:688-692, 1975.

[36] P. K. Currie and G. P. MacSithigh. The stability and dissipation of solutions for shearing flow of nematic liquid crystals. The Quarterly Journal of Mechanics and Applied Mathematics, 32:499-511, 1979.

[37] P. G. de Gennes. The Physics of Liquid Crystals. Clarendon Press, 1974. 
[38] P. G. de Gennes and J. Prost. The Physics of Liquid Crystals, 2nd Edition. Oxford University press, 1993.

[39] W. H. de Jeu. Physical Properties of Liquid Crystalline Materials. Gordon and Breach, New York, 1980.

[40] J. A. Diez and L. Kondic. Computing three-dimensional thin film flows including contact lines. Journal of Computational Physics, 183(1):274-306, 2002.

[41] M. Doi and S. F. Edwards. The theory of polymer dynamics. Clarendon Press, Oxford, 1986.

[42] J. L. Doricio. Gensmac - sof: Um método numérico para simular escoamentos incompressíveis de fluidos de segunda ordem. Master's thesis, Universidade de São Paulo, Instituto de Ciências Matemáticas e Computação, São Carlos, 2003.

[43] D. A. Dunmur, A. Fukuda, and G. R. Luckhurst (Eds). Physical Properties of Liquid Crystals: Nematics. EMIS Datareviews Series No. 25, The Instituition of Electrical Engineers (INSPEC), The Instituition of Electrical Engineers (INSPEC), London, 2001.

[44] R. Eidenschink, G. Konrath, H. Kretzschmann, and M. Rombach. Unusual lift by shearing mesogenic fluids. Molecular Crystals and Liquid Crystals, 330:327, 1999.

[45] J. L. Ericksen. Anisotropic fluids. Archive for Rational Mechanics and Analysis, 4:231-237, 1960.

[46] J. L. Ericksen. Conservation laws for liquid crystals. Transactions of the Society of Rheology, 5:23-24, 1961.

[47] J. L. Ericksen. Liquid crystals with variable degree of orientation. Archive for Rational Mechanics and Analysis, 113:97-120, 1991.

[48] T. E. Faber. Fluid Dynamics for Physicists. Cambridge University Press, Cambridge, 1995.

[49] J. Feng and L. G. Leal. Pressure-driven channel flows of a model liquid-crystalline polymer. Physics of Fluids, 11:2821-2835, 1999.

[50] V. G. Ferreira, A. C. Brandi, F. A. Kurokawa, P. Seleghim, A. Castelo, and J. A. Cuminato. Incompressible turbulent flow simulation using $\kappa-\varepsilon$ model and upwind schemes. Mathematical Problems in Engineering, 2007:1-26, 2007.

[51] V. G. Ferreira, F. A. Kurokawa, C. M. Oishi, M. K. Kaibara, A. Castelo, and J. A. Cuminato. Evaluation of a bounded high order upwind scheme for $3 \mathrm{~d}$ incompressible free 
surface flow computations. Mathematics and Computers in Simulation, 79:1895-1914, 2009 .

[52] V. G. Ferreira, F. A. Kurokawa, R. A. B. Queiroz, M. K. Kaibara, C. M. Oishi, J. A. Cuminato, A. Castelo, M. F. Tomé, and S. McKee. Assessment of a high-order finite difference upwind scheme for the simulation of convection-diffusion problems. International Journal for Numerical Methods in Fluids, 60:1-26, 2009.

[53] V. G. Ferreira, N. Mangiavacchi, M. F. Tomé, A. Castelo, J. A. Cuminato, and S. McKee. Numerical simulation of turbulent free surface flow with two-equation $\kappa-\varepsilon$ eddy-viscosity models. International Journal for Numerical Methods in Fluids, 44:347-375, 2004.

[54] V. G. Ferreira, C. M. Oishi, F. A. Kurokawa, M. K. Kaibara, J. A. Cuminato, A. Castelo, M. F. Tomé, N. Mangiavacchi, and S. McKee. A combination of implicit and adaptative upwind tools for the numerical solution of incompressible free surface flows. Communications in Numerical Methods in Engineering, 23:419-445, 2007.

[55] M. G. Forest, S. Heidenreich, S. Hess, X. Yang, and R. Zhou. Dynamic texture scaling of sheared nematic polymers in the large ericksen number limit. Journal of Non-Newtonian Fluid Mechanics, 165:687-697, 2010.

[56] M. G. Forest, R. Zhou, and Q. Wang. Scaling behavior of kinetic orientational distributions for dilute nematic polymers in weak shear. Journal of Non-Newtonian Fluid Mechanics, 116:183-204, 2004.

[57] F. C. Frank. On the theory of liquid crystals. Discussions of the Faraday Society, 25:19-28, 1958.

[58] Fraunhofer-Gesellschaft. Almost frictionless gears with liquid crystal. ScienceDaily, 2008.

[59] V. Freedericksz and V. Zolina. Forces causing the orientation of an anisotropic liquid. Transactions of the Faraday Society, 29:919-930, 1933.

[60] G. Friedel. Les états mésomorphes de la matière. Annales de Physique, 18:273-474, 1922.

[61] Shufang Fu, Tomohiro Tsuji, and Shigeomi Chono. Effect of magnetic fields on out-of-plane orientations of nematic liquid crystalline polymers under simple shear flow. Molecular Physics, 107:245-252, 2009.

[62] T. Geelhaar. Liquid crystals for display applications. Liquid Crystals, 24:91-98, 1998.

[63] G. W. Gray, K. J. Harrison, and J. A. Nash. New family of nematic liquid crystals for displays. Electronics Letters, 9:130-131, 1973. 
[64] G. W. Gray, K. J. Harrison, J. A. Nash, J. Constant, D. S. Hulme, J. Kirton, and E. P. Raynes. Stable, low melting nematogens of positive dielectric anisotropy for displays devices. In J. F. Johnson and R. S. Porter (Eds), editors, in Liquid Crystals and Ordered Fluids, pages (2), 617-643. Plenum Press, New York, 1974.

[65] G. Goldbeck-Wood H. Tu and A. H. Windle. Simulation of texture evolution for nematic liquid crystalline polymers under shear flow. Liquid Crystals, 29:335-345, 2002.

[66] I. Haller. Elastic constants of the nematic liquid crystalline phase of p-Methoxybenzylidene-p-n-Butylaniline (MBBA). The Journal of Chemical Physics, 57:1400-1405, 1972 .

[67] W. H. Han and A. D. Rey. Supercritical bifurcations in simple shear flow of a non-aligning nematic: reactive parameter and director anchoring effects. Journal of Non-Newtonian Fluid Mechanics, 48:181-210, 1993.

[68] F. H. Harlow and J. E. Welch. Numerical calculation of time-dependent viscous incompressible flow of fluid with free surface. Physics of Fluids, 8:2182-2189, 1965.

[69] H. Heuer, H. Kneppe, and F. Schneider. Flow of a nematic liquid crystal around a cylinder. Molecular Crystals and Liquid Crystals, 200:51-70, 1991.

[70] C. W. Hirt, A. A. Amsden, and J. L. Cook. An arbitrary lagrangian-eulerian computing method for all flow speeds. Journal of Computational Physics, 14:227-253, 1974.

[71] W. V. D. Hodge. The Theory and Applications of Harmonic Integrals. Cambridge University Press, Cambridge, 1952.

[72] J. D. Jackson. Classical Electrodynamics. 3rd Edition, Wiley, New York, 1998.

[73] J. T. Jenkins. Flows of nematic liquid crystals. Annual Review of Fluid Mechanics, 10:197-219, 1978.

[74] H. Kelker. History of liquid crystals. Molecular Crystals and Liquid Crystals, 21:1-48, 1973.

[75] H. Kneppe and F. Schneider. Determination of the viscosity coefficients of the liquid crystal mbba. Molecular Crystals and Liquid Crystals, 65:23-38, 1981.

[76] H. Kneppe, F. Schneider, and N. K. Sharma. A comparative study of the viscosity coefficients of some nematic liquid crystals. Berichte Bunsengesellschaft für Physikalische Chemie, 85:784-789, 1981.

[77] H. Kneppe, F. Schneider, and N. K. Sharma. Rotational viscosity $\gamma_{1}$ of nematic liquid crystals. The Journal of Physical Chemistry, 77:3203-3208, 1982. 
[78] L. Kondic. Instabilities in gravity driven flow of thin fluid films. SIAM Reviews, 45(1):95-115, 2003.

[79] S. Kumar. Liquid crystals: experimental study of physical properties and phase transitions. Cambridge University press, 2001.

[80] O. Lehmann. Über fliessende krystalle. Zeitschrift fur Physikalische Chemie, 4:462-472, 1889 .

[81] F. M. Leslie. Some constitutive equations for anisotropic fluids. The Quarterly Journal of Mechanics and Applied Mathematics, 19:357-370, 1966.

[82] F. M. Leslie. Some constitutive equations for liquid crystals. Archive for Rational Mechanics and Analysis, 28:265-283, 1968.

[83] F. M. Leslie. Theory of flow phenomena in liquid crystals. Advances in Liquid Crystals, 4:1-81, 1979.

[84] C. M. Lin, P. Neogi, and R. M. Ybarra. Statics and dynamics of contact lines of nematic liquid crystals on mica. Chem. Eng. Sci., 55:37 - 42, 2000.

[85] G. P. MacSithigh and P. K. Currie. Apparent viscosity during simple shearing flow of nematic liquid crystals. Journal of Physics D: Applied Physics, 10:1471-1478, 1977.

[86] N. Mangiavacchi, A. Castelo, M. F. Tomé, J. A. Cuminato, M. L. B. de Oliveira, and S. McKee. An effective implementation of surface tension using the marker-and-cell method for axisymmetric and planar flows. SIAM Journal on Scientific Computing, 26:1340-1368, 2005.

[87] A. F. Martins. Measurement of viscoelastic coefficients for nematic mesophases using magnetic resonance, section 8.3 in reference [43].

[88] S. McKee, M. F. Tomé, V. G. Ferreira, J. A. Cuminato, A. Castelo, F. S. Souza, and N. Mangiavacchi. The mac method. Computers \& Fluids, 37:907-930, 2008.

[89] M. Miesowicz. Der einflu $\beta$ des magnetischen feldes auf die viskosität der flüssigkeiten in der nematischen phase. Bulletin of the Polish Academy of Sciences Mathematics, A:228-247, 1936.

[90] M. Miesowicz. The three coefficients of viscosity of anisotropic liquids. Nature, 158:27, 1946.

[91] M. Miesowicz. Liquid crystals in my memories and now - the role of anisotropic viscosity in liquid crystal research. Molecular Crystals and Liquid Crystals, 97:1-11, 1983. 
[92] H. Miyata. Finite-difference simulation of breaking waves. Journal of Computational Physics, 65:179-214, 1986.

[93] G. Mompean and M. Deville. Unsteady finite volume of oldroyd-b fluid through a three-dimensional planar contraction. Journal of Non-Newtonian Fluid Mechanics., 72:253-279, 1997.

[94] J. K. Moscicki. Measurement of viscosities in nematics, section 8.2 in reference [43].

[95] B. M. Moskowitz. Fundamental physical constants and conversion factors. In T. J. Ahrens (Ed.), editor, in Global Earth Physics: A Handbook of Physical Constants, pages 346-355. American Geophysical Union, Washington DC, 1995.

[96] N. J. Mottram and S. J. Hogan. Multiple solutions in twisted nematic liquid crystal layers. Continuum Mechanics and Thermodynamics, 9:213-228, 1997.

[97] S. B. G. O'Brien and L. W. Schwartz. Theory and modeling of thin film flows. In In Encyclopedia of Surface and Colloid Sci., pages 5283-5297. Marcel Dekker, 2002.

[98] H. Ockendon and J. R. Ockendon. Viscous Flow. Cambridge University Press, Cambridge, 1995.

[99] J. Oliveira. Desenvolvimento de um sistema de simulação de escoamentos de fluidos com superfícies livres bidimensionais. Master's thesis, Universidade de São Paulo, Instituto de Ciências Matemáticas e Computação, São Carlos, 2002.

[100] C. W. Oseen. Beiträge zur theorie der anisotropen flüssigkeiten. Archiv För Matematik, Astronomi Och Fysik, 19A:part 9, 1-19, 1925.

[101] C. W. Oseen. "flüssign kristalle, tatsachen theorien". Fortschr. d. Chemie, Eucken. A. Ed., 20, 1929.

[102] C. W. Oseen. The theory of liquid crystals. Transactions of the Faraday Society, 29:883-889, 1933.

[103] O. Parodi. Stress tensor for a nematic liquid crystal. Journal de Physique, 31:581-584, 1970.

[104] T.N. Phillips and A.J. Williams. Viscoelastic flow through a planar contraction using a semi-lagrangian finite volume method. Journal of Non-Newtonian Fluid Mechanics., 87:215-246, 1999.

[105] P. Pieranski and E. Guyon. Transverse effects in nematic flows. Physics Letters, 49A:237-238, 1974. 
[106] S. A. Pikin. Couette flow of a nematic liquid. Soviet Physics-JETP, 38:1246-1250, 1974.

[107] F. Reinitzer. " Beiträge zur Kenntniss des Cholesterins ". Monatshefte Für Chemie/Chemical Monthly, 9:421-441, 1888.

[108] F. Reinitzer. Contributions to the knowledge of cholesterol. translation of reference [107]. Liquid Crystals, 5:7-18, 1889.

[109] A. D. Rey. The neumann and young equations for nematic contact lines. Liquid Crystals, 27:195-200, 2000.

[110] A. D. Rey and M. M. Denn. Jeffrey-hamel flow of leslie-ericksen nematic liquids. Journal of Non-Newtonian Fluid Mechanics, 27:375-401, 1988.

[111] A. D. Rey and M. M. Denn. Dynamical phenomena in liquid-crystalline materials. Annual Review of Fluid Mechanics, 34:233-266, 2002.

[112] A. D. Rey and T. Tsuji. Recent advances in theoretical liquid crystal rheology. Macromolecular Theory and Simulations, 7:623-639, 1998.

[113] P. Romano and E. G. Virga. Adhesion of oriented nematic droplets. Continuum Mech. Thermodyn., 12:363 - 378, 2000.

[114] T. Sato and S.M. Richardson. Explicit numerical simulation of time-dependent viscoelastic flows problems by a finite element/finite volume method. Journal of Non-Newtonian Fluid Mechanics., 51:249-275, 1994.

[115] A. Saupe. On molecular structure and physical properties of thermotropic liquid crystals. Molecular Crystals and Liquid Crystals, 7:59-74, 1969.

[116] F. Schneider and H. Kneppe. Flow phenomena and viscosity. In D. Demus, J. Goodby, G. W. Gray, H. W. Spiess, and V. Vill, editors, in Handbook of Liquid Crystals, volume 1, pages 454-476. Wiley-VCH, Weinheim, Germany, 1998.

[117] L. W. Schwartz. Unsteady simulation of viscous thin-layer flows. In Free-Surface Flows with Viscosity, editor, In P. Tyvand, pages 203-233. Computational Mech. Publ., 1997.

[118] K. Skarp and T. Carlsson. Influence of an electric field on the flow alignment angle in shear flow of nematic liquid crystals. Molecular Crystals and Liquid Crystals, 49:75-82, 1978.

[119] A. A. Sonin. The surface physics of liquid crystals. Gordon and Breach, 1995.

[120] M. J. Stephen and J. P. Straley. Physics of liquid crystals. Reviews of Modern Physics, 46:617-704, 1974. 
[121] M. J. Stephen and J. P. Straley. Physics of liquid crystals. Reviews of Modern Physics, 46:617-704, 1974.

[122] F. Stewart and I. W. Stewart. A novel method for measuring compression constants in smectics. Molecular Crystals and Liquid Crystals, 478:779 - 788, 2007.

[123] I. W. Stewart. The Static and Dynamic Continuum Theory of Liquid Crystals. Taylor and Francis, London, 2004.

[124] J. Tao and J. J. Feng. Effects of elastic anisotropy on the flow and orientation of sheared nematic liquid crystals. Journal of Rheology, 47:1051-1070, 2003.

[125] T. Temam. Sur l'approximation de la solution des equations de navier-stokes par la methode de pas fractionnaires (ii). Archieves of Rational Mechanics and Analysis, 33:377-385, 1969.

[126] M. F. Tomé, M. S. B. Araujo, M. A. Alves, and F. T. Pinho. Numerical simulation of viscoelastic flows using integral models: a finite difference approach. Journal of Computational Physics, 227:4207-4243, 2008.

[127] M. F. Tomé, A. Castelo, J. A. Cuminato, N. Mangiavacchi, and S. McKee. Gensmac3d: A numerical method for solving unsteady three-dimensional free surface flows. International Journal for Numerical Methods in Fluids, 37 (7):747-796, 2001.

[128] M. F. Tomé, A. Castelo, V. G. Ferreira, and S. McKee. A finite difference technique for solving the oldroyd-b model for 3d-unsteady free surface flows. Journal of Non-Newtonian Fluid Mechanics, 154:179-206, 2008.

[129] M. F. Tomé, J. L. Doricio, J. A. Cuminato, A. Castelo, and S. McKee. Solving viscoelastic free surface flows of a second-order fluid using a marker-and-cell approach. International Journal for Numerical Methods in Fluids, 53:599-627, 2007.

[130] M. F. Tomé, J. L. Doricio, J. A. Cuminato, A. Castelo, and S. McKee. Solving viscoelastic free surface flows of a second order fuid using a marker-and-cell approach. International Journal for Numerical Methods in Fluids, 53:599-627, 2007.

[131] M. F. Tomé, B. Duffy, and S. McKee. A numerical technique for solving unsteady non-newtonian free surface flows. Journal of Non-Newtonian Fluid Mechanics, 62:9-34, 1996.

[132] M. F. Tomé, L. Grossi, A. Castelo, J. A. Cuminato, S. McKee, and K. Walters. Die-swell, splashing drop and a numerical technique for solving the oldroyd-b model for axisymmetric free surface flows. Journal of Non-Newtonian Fluid Mechanics, 141:147-165, 2007. 
[133] M. F. Tomé, N. Mangiavacchi, J. A. Cuminato, A. Castelo, and S. McKee. A finite difference technique for simulating unsteady viscoelastic free surface flows. Journal of Non-Newtonian Fluid Mechanics, 106:61-106, 2002.

[134] M. F. Tomé and S. McKee. Gensmac: A computational marker-and-cell method for free surface flows in general domains. Journal of Computational Physics, 110:171-186, 1994.

[135] C. Truesdell and W. Noll. The non-linear field theories of mechanics. In S. Flügge (Ed.), editor, in Handbuch der Physik, volume III/3, page 526. Springer-Verlag, Berlin, 1965.

[136] F. VandenBrouck, M. P. Valignat, and A. M. Cazabat. Thin nematic films: metastability and spinodal dewetting. Physic Review Letters, 82 (13):2693 - 2696, 1999.

[137] E. G. Virga. Drops of nematic liquid crystals. Arch. Rational Mech. Ana., 107:371 - 390, 1989.

[138] E. G. Virga. Variational Theories of Liquid Crystals. Chapman and Hall, London, 1994.

[139] A. J. Walker and I. W. Stewart. Poiseuille flow of a smectic a liquid crystal. International Journal of Engineering Science, 48:1961 - 1970, 2010.

[140] K. Walters and M. F. Webster. The distinctive cfd challenges of computational rheology. International Journal for Numerical Methods in Fluids, 43:577-596, 2003.

[141] J. E. Welch, F. H. Harlow, J. P. Shannon, and B. J. Daly. The mac method. Tech. report, Los Alamos Scientific Laboratory Report LA-3245, Los Alamos, 1965.

[142] S. A. White, A. Gotsis, and D. G. Baird. Review of the entry flow problem: Experimental and numerical. Journal of Non-Newtonian Fluid Mechanics, 24:121-160, 1987.

[143] T. Yamamoto, Y. Yamasaki, Y. Tanaka, and N. Mori. Three-dimensional flow of liquid crystalline polymers through rectangular channels with abrupt change in geometry. Fluid Dynamics Research, 38:452 - 468, 2006.

[144] H. Yu and P. Zhang. A kinetic-hydrodynamic simulation of microstucture of liquid crystal polymers in plane shear flow. Journal of Non-Newtonian Fluid Mechanics, 141:116-127, 2007.

[145] H. Zhou and H. Wang. Elongational perturbations on nematic liquid crystal polymers under a weak shear. Physics of fluids, 19:103-107, 2007.

[146] H. Zocher. Über die einwirkung magnetischer, elektrischer und mechanischer kräfte auf mesophasen. Physik. Zietschr., 28:790-796, 1927. 


\section{Tabelas Importantes}

Neste apêndice são apresentadas quatro importantes tabelas para esta tese.

\begin{tabular}{|c|c|c|c|}
\hline Grandezas & Símbolo & Unidades Gaussianas & Unidades SI \\
\hline densidade & $\rho$ & $1 \mathrm{~g} \mathrm{~cm}^{-3}$ & $10^{3} \mathrm{~kg} \mathrm{~m}^{-3}$ \\
\hline volume específico & $\nu=1 / \rho$ & $1 \mathrm{~cm}^{3} \mathrm{~g}^{-1}$ & $10^{-3} \mathrm{~m}^{3} \mathrm{~kg}^{-1}$ \\
\hline anisotropia magnética & $\Delta \chi$ & 1 & $4 \pi$ \\
\hline anisotropia dielétrica & $\epsilon_{a}$ & 1 & 1 \\
\hline força & $\mathrm{F}$ & 1 dyn & $10^{-5} \mathrm{~N}$ \\
\hline tensão superficial & $\gamma$ & 1 dyn $\mathrm{cm}^{-1}$ & $10^{-3} \mathrm{~N} \mathrm{~m}^{-1}$ \\
\hline pressão & $p$ & 1 dyn $\mathrm{cm}^{-2}$ & $10^{-1} \mathrm{~Pa}$ \\
\hline viscosidade dinâmica & $\alpha_{i}, \eta_{i}, \gamma_{i}$ & $1 \mathrm{P}$ & $10^{-1} \mathrm{~Pa} . \mathrm{s}$ \\
\hline indução magnética & $\mathrm{B}$ & $1 \mathrm{G}$ & $10^{-4} \mathrm{~T}$ \\
\hline campo magnético & $H$ & $1 \mathrm{Oe}$ & $\frac{1}{4 \pi} 10^{3} \mathrm{~A} \mathrm{~m}^{-1}$ \\
\hline potencial elétrico & V & 1 statvolt & $10^{-6} c \mathrm{~V}$ \\
\hline campo elétrico & $\mathrm{E}$ & 1 statvolt $\mathrm{cm}^{-1}$ & $10^{-4} c \mathrm{~V} \mathrm{~m}^{-1}$ \\
\hline energia & $W$ & $1 \mathrm{erg}$ & $10^{-7} \mathrm{~J}$ \\
\hline densidade de energia & $\underline{w}$ & $1 \mathrm{erg} \mathrm{cm}^{-3}$ & $10^{-1} \mathrm{~J} \mathrm{~m}^{-3}$ \\
\hline
\end{tabular}

Tabela A.1: Fatores de conversão para grandezas[72, 95]. Esta tabela permite simples conversões das unidades cgs Gaussianas para unidades no sistema SI. As abreviações usadas foram: $(\mathrm{P})$ poise, $(\mathrm{G})$ gauss, (Oe) oersted, $(\mathrm{N})$ newton, (Pa s) pascal segundo, $(\mathrm{T})$ tesla , $(\mathrm{A})$ ampère, $(\mathrm{V})$ volt, $(\mathrm{J})$ joule. $c \doteqdot 2.998 \times 10^{8} \mathrm{~m} \mathrm{~s}^{-1}$ representa o valor numérico da velocidade da luz no sistema SI. 


\begin{tabular}{|c|c|c|c|}
\hline Grandezas & MBBA perto $25^{\circ} \mathrm{C}$ & PAA perto $122^{\circ} \mathrm{C}$ & $5 \mathrm{CB}$ perto $26^{\circ} \mathrm{C}$ \\
\hline $\begin{array}{l}\text { viscosidades (Pa s) } \\
\alpha_{1} \\
\alpha_{2} \\
\alpha_{3} \\
\alpha_{4} \\
\alpha_{5} \\
\alpha_{6}\end{array}$ & $\begin{array}{l}-0.0181[77] \\
-0.1104[77] \\
-0.001104[77] \\
0.0826[77] \\
0.0779[77] \\
-0.0336[77]\end{array}$ & $\begin{array}{r}0.0043[121] \\
-0.0069[121] \\
-0.0002[121] \\
0.0068[121] \\
0.0047[121] \\
-0.0023[121]\end{array}$ & $\begin{array}{r}-0.0060 \\
-0.0812 \\
-0.0036 \\
0.0652 \\
0.0640 \\
-0.0208\end{array}$ \\
\hline $\begin{array}{l}\eta_{1} \\
\eta_{2} \\
\eta_{3} \\
\eta_{12} \\
\gamma_{1} \\
\gamma_{2}=\eta_{1}-\eta_{2}\end{array}$ & $\begin{array}{c}0.0240[75] \\
0.1361[75] \\
0.0413[75] \\
-0.0181[75] \\
0.1093[77] \\
-0.1121\end{array}$ & $\begin{array}{l}0.0024[90] \\
0.0092[90] \\
0.0034[90] \\
0.0043[121] \\
0.0067[121] \\
-0.0068\end{array}$ & $\begin{array}{r}0.0204[76] \\
0.1052[76] \\
0.0326[76] \\
-0.0060[76] \\
0.0777[77] \\
-0.0848\end{array}$ \\
\hline $\begin{array}{l}\text { constantes elásticas (N) } \\
K_{1} \\
K_{2} \\
K_{3}\end{array}$ & $\begin{array}{r}6 \times 10^{-12}[66] \\
3.8 \times 10^{-12}[66] \\
7.5 \times 10^{-12}[66] \\
\end{array}$ & $\begin{array}{r}6.9 \times 10^{-12}[39] \\
3.8 \times 10^{-12}[39] \\
11.9 \times 10^{-12}[39] \\
\end{array}$ & $\begin{array}{l}6.2 \times 10^{-12}[43, \text { p. } 220] \\
3.9 \times 10^{-12}[43, \text { p. } 220] \\
8.2 \times 10^{-12}[43, \text { p. } 220]\end{array}$ \\
\hline $\begin{array}{l}\text { tensão superficial }\left(\mathrm{N} \mathrm{m}^{-1}\right) \\
\gamma\end{array}$ & $35.5 \times 10^{-3}[43$, p. 487$]$ & $38 \times 10^{-3}[121]$ & $32.6 \times 10^{-3}[43$, p. 488$]$ \\
\hline $\begin{array}{l}\text { constantes dielétricas } \\
\epsilon_{\|} \\
\epsilon_{\perp} \\
\epsilon_{a}=\epsilon_{\|}-\epsilon_{\perp} \text { (adimensional) }\end{array}$ & $\begin{array}{l}4.7[121] \\
5.4[121] \\
-0.7\end{array}$ & $\begin{array}{l}5.538[121] \\
5.705[121] \\
-0.167\end{array}$ & $\begin{array}{c}18.5[43, \mathrm{p} .525] \\
7[43, \mathrm{p} .525] \\
11.5[43, \mathrm{p} .525]\end{array}$ \\
\hline $\begin{array}{l}\text { anisotropia magnética } \\
\Delta \chi(\text { adimensional) unidades SI } \\
\chi_{a} \text { unidades } \operatorname{cgs}\end{array}$ & $\begin{array}{l}1.219 \times 10^{-6} \\
0.97 \times 10^{-7}[121]\end{array}$ & $\begin{array}{l}1.48 \times 10^{-6} \\
1.18 \times 10^{-7}[121]\end{array}$ & $\begin{array}{l}1.43 \times 10^{-6} \\
1.14 \times 10^{-7}[16]\end{array}$ \\
\hline $\begin{array}{l}\text { densidade }\left(\mathrm{kg} \mathrm{m}^{-3}\right) \\
\rho\end{array}$ & 1088 [121] & 1168 [121] & 1020 \\
\hline $\begin{array}{l}\text { volume específico }\left(\mathrm{m}^{3} \mathrm{~kg}^{-1}\right) \\
v=1 / \rho\end{array}$ & $9.19 \times 10^{-4}$ & $8.56 \times 10^{-4}$ & $9.8 \times 10^{-4}[43$, p. 154$]$ \\
\hline$T_{N I}$ & $45.1^{\circ} \mathrm{C}[77]$ & $135.5^{\circ} \mathrm{C}[39]$ & $35.4^{\circ} \mathrm{C}[77]$ \\
\hline
\end{tabular}

Tabela A.2: Valores de parâmetros físicos para as fases nemáticas de MBBA, PAA e 5CB no sistema de unidades SI; os valores de $\chi_{a}$ também são mostrados no sistema de unidades cgs. A temperatura de transição de nemático para isotrópico é denotada por $T_{N I}$. As viscosidades para 5CB foram deduzidas de uma tabela fornecida por [76] e [75] utilizando fórmulas de conversão padronizadas [123]. 


\begin{tabular}{||c||c|c|c||}
\hline Constantes Fundamentais & Símbolo & Unidades Gaussianas & Unidades SI \\
\hline \hline Velocidade da Luz & $c$ & $2.998 \times 10^{10} \mathrm{~cm} \mathrm{~s}^{-1}$ & $2.998 \times 10^{8} \mathrm{~m} \mathrm{~s}^{-1}$ \\
Permeabilidade do espaço livre & $\mu_{0}$ & 1 & $4 \pi \times 10^{-7} \doteqdot 12.566 \times 10^{-7} \mathrm{H} \mathrm{m}^{-1}$ \\
Permissividade do espaço livre & $\epsilon_{0}$ & 1 & $\mu_{0}^{-1} c^{-2} \doteqdot 8.854 \times 10^{-12} \mathrm{~F} \mathrm{~m}^{-1}$ \\
Aceleração da gravidade & $g$ & $980.665 \mathrm{~cm} \mathrm{~s}^{-2}$ & $9.80665 \mathrm{~m} \mathrm{~s}^{-2}$ \\
\hline
\end{tabular}

Tabela A.3: Constantes Fundamentais. As abreviações utilizadas são: henry (H), farad (F), ampère $(\mathrm{A})$ 